Florida International University FIU Digital Commons

FIU Electronic Theses and Dissertations

University Graduate School

4-1999

\title{
Jazz band arranging : original big band arrangements
}

Jay M. Byron

Florida International University

DOI: $10.25148 /$ etd.FI14052513

Follow this and additional works at: https://digitalcommons.fiu.edu/etd

Part of the Composition Commons

\section{Recommended Citation}

Byron, Jay M., "Jazz band arranging : original big band arrangements" (1999). FIU Electronic Theses and Dissertations. 1950. https://digitalcommons.fiu.edu/etd/1950

This work is brought to you for free and open access by the University Graduate School at FIU Digital Commons. It has been accepted for inclusion in FIU Electronic Theses and Dissertations by an authorized administrator of FIU Digital Commons. For more information, please contact dcc@fiu.edu. 


\title{
FLORIDA INTERNATIONAL UNIVERSITY \\ Miami, Florida
}

\author{
JAZZ BAND ARRANGING \\ Original Big Band Arrangements
}
A project submitted in partial fulfillment of the
requirements for the degree of
MASTER OF MUSIC
in
JAZZ STUDIES
by

Jay M. Byron

April 1999 


\section{TABLE OF CONTENTS}

PREFACE. .3

\section{CHAPTER}

I. THIS I DIG OF YOU

Overview

Performance Considerations

II. THE WAY YOU LOOK TONIGHT

Overview

Performance Considerations

III. WHEN SUNNY GETS BLUE...........................................9

Overview

Performance Considerations

IV. UPSIDE, DOWNSIDE.................................................11 Overview

Performance Considerations

V. DOLPHIN DANCE....................................................13

Analysis

Performance Considerations

VI. APPENDIX ...............................................................16

Samples of Arranging Techniques

VII. SCORES 


\section{Preface}

This paper examines five big band arrangements written during a period of two semesters from 1998-1999. I will provide an overview and performance considerations for each arrangement.

Each arrangement uses common conventions such as unison lines, octave doubling, four and five part voicings, found in closed, semi-open, and open position. Approach techniques include diatonic, dominant, diminished, chromatic, and parallel. Choice was based primarily on two considerations: desired texture and the best voice leading options identified to provide each part with a swinging line and maintain melodic integrity. Other conventions applied include chord substitution, upper structure triads, and altered and diminished scales to provide harmonic contrast and color.

Each arrangement supplied new challenges and the tunes selected provided the arranger with a diverse experience of styles. The inherent qualities of the melody and harmonic progression of each piece were the primary considerations for selection. 


\section{CHAPTER I \\ THIS I DIG OF YOU}

\section{Overview}

This I Dig of You is a Hank Mobley composition in B flat major originally recorded on the Blue Note recording Soul Station. It is an up-tempo swing tune in ABAB', 32 measure format. The arrangement has an 8 measure introduction followed by the $\mathrm{A}$ theme presented by a unison sax line. The B section is then answered by trumpets and trombones in four way closed position. The A section is then restated by the saxophones in four way double lead and once again the trumpets and trombones answer with a truncated 6 measure version of the $\mathrm{B}$ theme. The final 2 bars of this 8 measure section lead into the sax soli section.

The sax soli is a transcription of Hank Mobley's first solo chorus on the Soul Station recording written in four way closed double lead and runs from measure 39-52. The trumpets and trombones using original material then provide a transition to the solo section.

The solo section changes to a hip-hop groove after being set up by the rhythm section. The solo section is open and is based on rhythm changes using tritone substitution. Layered backgrounds are provided and build gradually. The second ending is a transition to a variation of the B theme with some original material. Once again the rhythm section establishes a contrasting time feel by employing an illusion of metric modulation through the displacement of accents. Once established, the ensemble enters with the A theme in tutti and is apparently in three. However, after stating the entire theme the band falls on a 
beat four accent and collectively re-establishes the meter as four. The trumpets and trombones then state the $\mathrm{B}$ theme for the last time while call and response melodies exchange between the brass and saxophones while delaying the final cadence and providing a more dramatic finish.

\section{Performance Considerations}

This is an up-tempo swing chart. It works best at about 200. The bass and bari sax need to establish a firm groove from the onset. The rhythm in measure 8 and landing square on beat one in measure 9 are essential for establishing the time feel for the sax entrance. The brass must use crisp and accurate articulation. Note placement might be difficult in certain parts as the altered scale choices provide a rather dense harmonic sonority. For the sake of the brass, these sections should be worked slowly with rhythm section to aid musicians in finding pitches. This will save rehearsal time and engrain pitch accuracy.

The solo section should be set-up by the drummer in measures 61-62. The rhythm section my groove before the entrance of the soloist or the soloist may enter immediately. This is optional. The changes for the solo section are based on rhythm changes while using tritone sub chords to create a chromatically descending bass line. Saxes enter on cue and repeat as trombones add hits and finally the trumpets with a bluesy lick that signals the final chorus.

The shout at measure 74 is tutti and requires accuracy and clean articulation, especially on the eighth notes on the up beat. Measure 80 is critical as it provides the send-off for the rhythm section to set-up the feel for the triple meter. The tutti section at measure 84 should crescendo until the tutti beat four accent in measure 89 . From this 
point, backgrounds should be sure to give way to melodic material and provide a convincing call and response effect. 


\section{CHAPTER II}

\section{THE WAY YOU LOOK TONIGHT}

\section{Overview}

The inspiration for the arrangement of this Jerome Kern compostion developed from listening to the Blowin' Sessions recording featuring Hank Mobley, Johnny Griffen, John Coltrane, and Lee Morgan.

The form of the tune is an extended AABA format with each section lasting 16 bars. The introduction and the end of each chorus is marked by a four bar ensemble pedal figure that was borrowed from the Blowin' Sessions recording. The saxophones introduce the $\mathrm{A}$ theme at measure 7 in octave voicing. The $\mathrm{A}$ theme then repeats at measure 23 in four-way closed, double lead with brass backgrounds extrapolated from melodic embellishment figures found on the recording. A trombone soli introduces the B theme at measure 39 in unison voicing which breaks into four-way closed in the fourth bar. The entire ensemble then joins at measure 46 to finish the B theme. Measure 55 rounds off the first chorus in full ensemble tutti.

A tenor solo feature at measure 75 runs a full chorus with backgrounds providing harmonic color from the second A section. The tenor solo finishes on the four bar pedal figure which not only gives closure to the solo, but provides a natural introduction to trombone and bass soli at measure 128 . The soli was inspired by the Rob McConnel style and written as an A theme variation utilizing unison voicing while breaking into four-way closed at key points of harmonic interest. The next A theme returns with the full ensemble. While trying to capture the essence of Lee Morgan, measure 160 features a 
written trumpet solo over the B theme which is subsequently joined in a trumpet duet in measure 168 and brings the $B$ theme to closure. Measure 176 is a full ensemble return to the A theme. The arrangement ends with a half-time re-harmonization of the A theme tag.

\section{Performance Considerations}

This arrangement is to be performed as up-tempo as possible however, some of the background figures will prove to be difficult at a tempo that exceeds 220. A strong lead trumpet is a requirement for this piece. The quarter note triplet figures at measure 57 need to be played legato and maintain steady tempo. The shift to the contrasting rhythm in measure 61 will need to be paid special attention.

The tenor solo background figures provide interesting harmonic color but must remain subdued so as to not interfere with the soloist. Background accents at measure 112 need to be precise, crisp, and supported by the snare drum. This section should crescendo gradually. The trombone soli section should swing and special attention should be given to the melodic embellishment. The trumpet solo requires the use of alternate fingerings $(1-3 / 1)$. The repeated notes lay more naturally if initiated with the alternate (1-3). Once again, backgrounds need to be subdued, however the trumpet should be encouraged to sing over the top with a full tone.

The last full ensemble $A$ theme should be big and swinging leading to the last two bars in half-time to be played big and fat! 


\section{CHAPTER III \\ WHEN SUNNY GETS BLUE}

\section{Overview}

This arrangement in the key of $\mathrm{F}$ is a vocal ballad written in quasi Basie style and follows the original AABA, 32 bar song format. The introduction opens with full ensemble in double-time and is derived from the second 4 bars of the A section. The vocalist enters in the ninth bar as the tempo drops into half-time and runs the first $A$ with rhythm section accompaniment only. Upon repeat, saxophone backgrounds enter providing more harmonic interest. The B section is then supported by trombone pads answered by flugel horns and subsequently the saxophones. A return to the A theme is once again provided with sax pads with a variation of rhythm and harmonic color.

Measure 33 returns to double-time with the trombones voiced in fourths and setting up one chorus of solo complete with backgrounds. The full ensemble shout in Basie style arrives at measure 53 and is interspersed with answers form the sax section. Measure 69 features the trumpets and trombones on the B theme in very tight four-way closed voicing with sax pads returning to half time through metric modulation in measure 83 using quarter note triplets. The vocalist then enters in measure 85 bringing the arrangement to conclusion with the A theme.

\section{Performance Considerations}

The tempo of this arrangement is critical to it's musical success. It will work best at 106-112 while providing enough impetus for the double-time sections and an almost rubato feel for the half time sections. The bass line pick-up must be played solidly with 
swing to establish the tempo and feel. Measure 5 of the introduction is a pyramid and must be executed accurately with accents in order to be convincing.

When the vocalist enters backgrounds must be played in subtone and very legato. The third trombone in measure 18 provides forward movement to the backgrounds and should be heard. Eighth notes at measure 19 need to be played straight at this tempo so play rhythms as written. Rhythms written into backgrounds are coordinated with the vocal melody so care must be exercised so that they are synchronized throughout.

The double time section at measure 33 should be played forte and articulated as indicated. Solo backgrounds at measure 45 must be played mezzo forte and will need to be rehearsed for accuracy. The fourth beat of measure 52 must be played with a fat accent to establish the feel of the swinging shout to follow. Measure 69 is voiced tightly and needs to swing hard. Do not allow sax pads get in the way.

For maximum effectiveness, the quarter note triplets at measure 83 should be exaggerated followed by very legato quarter notes in measure 84 to return to half time feel. Measure 93 supports the repeated tag of the vocalist. Beat three should not arrive until the vocalist lands on the lyric "Blue." 


\section{CHAPTER IV \\ UPSIDE, DOWNSIDE}

\section{Overview}

Upside, Downside is a Mike Stern composition in e minor from the album of the same title recorded in 1986 . Originally recorded by a fusion ensemble, this arrangement is an adaptation for big band with the intention of maintaining the original sonority and intensity.

The main theme of the tune is the counterpoint interplay between an ostinato in the bass and a very angular melody played by the guitar (electronic wind instrument or tenor sax) and reflects the tendencies of bass lines of progressions utilizing tritone substitutions. The full ensemble playing a syncopated, harmonically dense pad marks the end of each section.

The melody continues to evolve and gains more momentum as the composition unfolds. The last eight measures of the exposition then fall into a "Weather Report" like groove bringing the arrangement to an open solo section.

The solo section uses the same bass ostinato as the theme. Backgrounds are provided by the saxes and trombones. The sax line is actually a solo riff transcribed form the original guitar solo and provides harmonic motion to bring variety to the redundancy of the ostinato.

The arrangement then returns to the B theme of the exposition and runs it's course identical to the first time. At the conclusion, the piece recapitulates and ends on the eight measure, dense harmonic, full ensemble pad with the last measure providing contrast to 
the tunes sonority of e minor by resolving to an $\mathrm{E}^{+} \mathrm{Maj} .{ }^{7}$ chord. Possibly a fusion equivalent to the principle of the "picardy third."

\section{Performance Considerations}

The rhythm section is of prime importance and should be able to maintain a steady, intense groove throughout the arrangement. The bass player should be able to provide a clear and defined tone while the slap and pop technique would be most effective if employed ad libitum at the bass player's discretion. Furthermore, a five or six string bass would add bottom and fullness to the ensemble sections by allowing the bassist to play an octave lower than written. The drummer should be encouraged to fill any static sections throughout as in a drum feature and in the style of Dave Weckl or drummers of similar fusion style.

The tutti sections needs to be accented and tight to keep them from dragging. The sonority should be intense and compact like a synthesizer, not spread and heavy. There is a tendency to swing these sections and this must be avoided. This is a fusion tune and it should not swing in the traditional sense. Trombone backgrounds at measure 60 should use vibrato and exaggerate the glissando.

The solo section is open and may feature any instrument. The open section remains over the e minor ostinato so the rhythm section must contribute dynamic and rhythmic contrast in order to provide interest and support for the soloist. The return to the theme and the rest of the arrangement should continue in the same style as the exposition to the end. The drummer should place emphasis on the ritardando in the penultimate measure and set-up. The last chord should be intense and held for at least a full bar. 


\section{CHAPTER V \\ DOLPHIN DANCE}

\section{Overview}

A Herbie Hancock composition found on the Maiden Voyage album, Dolphin Dance is a through-composed, modal piece centered around the key of $\mathrm{c}$ dorian. Much of the material for this arrangement was inspired by the unique voicing characteristics of Hancock's style. However, some original melodic material was used as well as contrasting time feel, (e.g., swing vs. even eighth notes), to add character and interest to the arrangement.

The introduction is based on the voicing of the $\mathrm{c} \#$ minor chord found in measure 29 of the tune. Treated in augmentation, the ostinato rhythm and the sonority of the chord set the mood for the arrangement. In addition, the shift from even to swing eighths sets the precedent for the character of the overall arrangement. The melody enters in measure 20 and starts as a unison duet between the third trumpet and second alto. At measure 28, with the addition of sax backgrounds the melody splits into harmony while emphasizing the chromatic line in the progression.

The soli, written for the sax section, is four-way, double lead harmonization of a full chorus of a Bill Evans transcription that uses some of the reharmonization preferences employed by Evans. The soli runs for a full chorus with brass backgrounds added at the midpoint. An open solo section immediately follows and runs for a full chorus. The solo choruses of Freddie Hubbard on the same recording inspired solo backgrounds.

The shout enters at measure 130 and is a permutation of the original melody. Further variation of the melody occurs at measure 142 although the chord progression maintains 
the integrity of the original. This section continues through the end of the chorus where it is met by a group of quarter note triplets in measure 161 which provide a vehicle for metric modulation into $3 / 4$ time in measure 162 .

A vamp in three sets up the last section which presents an entire chorus of the melody, also in three, utilizing the call and respose phrasing between the saxes and brass. The tune finally comes to rest on a variation of the introduction in three and is open for improvisation with backgrounds played on cue.

\section{Performance Considerations}

The introduction must be played in even eighths with emphasis given to the bass line in the bass and baritone sax. The swing feel should be set-up by the drums and doubled bass line that occurs in measure 9 and continue throughout the rest of the piece until otherwise indicated.

The melody at measure 20 should by played on flugel horn and alto sax. Backgrounds should be played in a subdued fashion and not interfere with the melody while emphasizing the alterations and tensions provided by the harmony.

It is necessary to play the sax soli in the hard swing style of Freddie Hubbard. Phrasing alike is critical and is suggested by the phraseology of the line itself. Again, backgrounds should be no louder than mezzo forte and executed with precision and clarity. Solo section backgrounds must swing without interrupting the open space and interpretation of the soloist.

The shout at measure 130 must swing hard and balance achieved throughout the

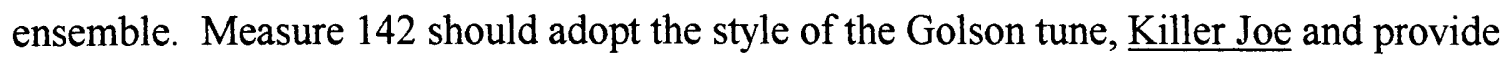
stylistic contrast to the even eighths feel of measures 146-149. 
The quarter note triplet figure in measure 161 must be played legato with heavy emphasis to successfully establish the triple meter. The bass ostinato in measures 166 169 may be opened up for solo before the last ensemble chorus. The last section of interplay between saxes and brass must be balanced and attention should be given to the unison lines in the brass as accuracy tends to be a problem. The last vamp is open and serves as a vehicle to feature yet another soloist. The backgrounds are unison and provide an opportunity to feature other ensemble members. This section should slowly build while the drummer becomes more aggressive. As the level rises, backgrounds can be added starting with the trombones and ultimately the unison call and response lines of the trumpets and saxes. 


\section{APPENDIX}

SAMPLES OF ARRANGING TECHNIQUES APPLIED

Technique

Unison Voicings

Four Part Double Lead

Five Part Drop 2

Spread Voicing

Diatonic Approach

Chromatic Approach

Parallel Approach

Diminished Approach

Dominant Approach

Upper Structure Triads

Voicings in Fourths

Chromatic Motion

Melodic Minor Harmony

Herbie Hancock Voicings

Altered Scale

Diminished Scale

Metric Modulation
Tune

The Way You Look Tonight Sax

The Way You Look Tonight Sax

$\operatorname{Sax}$

Dolphin Dance

Dolphin Dance

Ensemble

Ensemble

53

When Sunny Gets Blue

The Way You Look Tonight Ensemble

123

This I Dig of You

Trombones $\quad 66$

Dolphin Dance

Sax

58

When Sunny Gets Blue

Sax/Trombone 69

Dolphin Dance

Ensemble $\quad 165$

When Sunny Gets Blue

Trombones

33

The Way You Look Tonight Ensemble

64

When Sunny Gets Blue

Sax

17

Dolphin Dance

Sax

115

This I Dig of You

Trumpets

58

Dolphin Dance

Sax

228

Dolphin Dance

Ensemble

161 


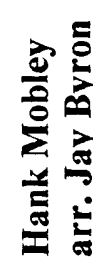
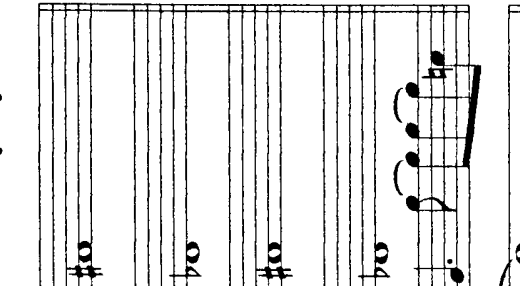

$\infty$

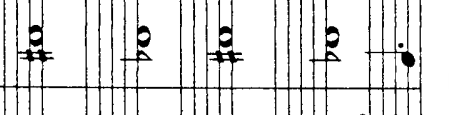

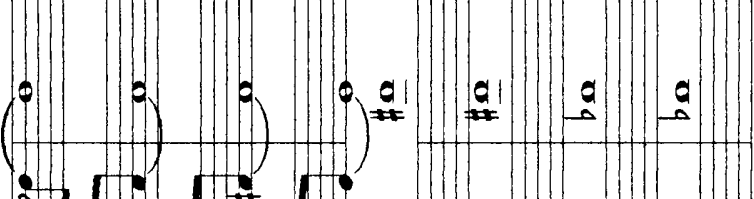

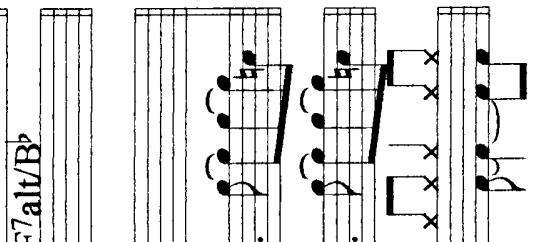

\section{(a)}

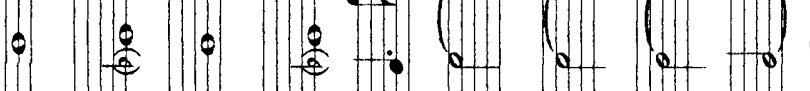
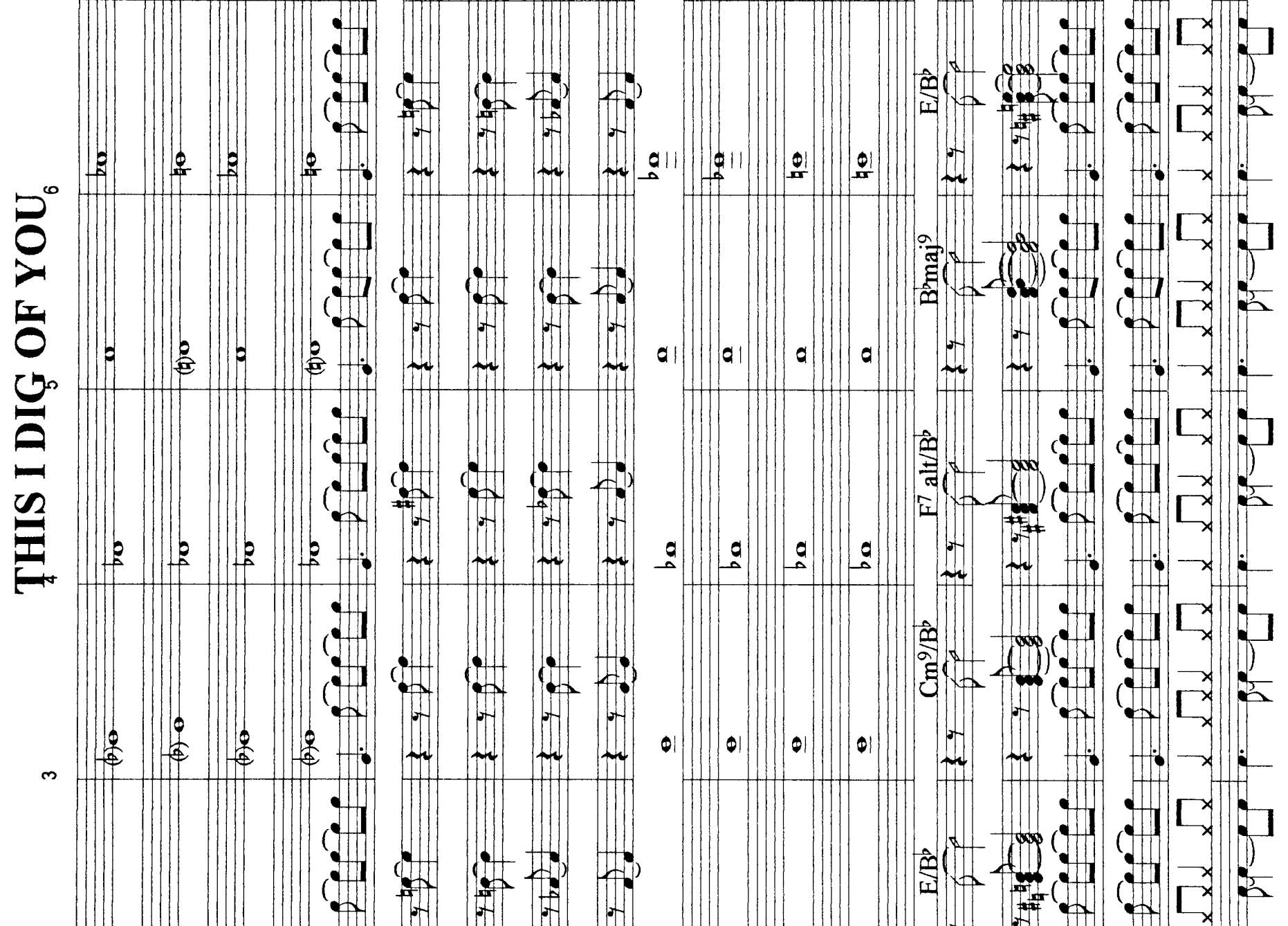

E

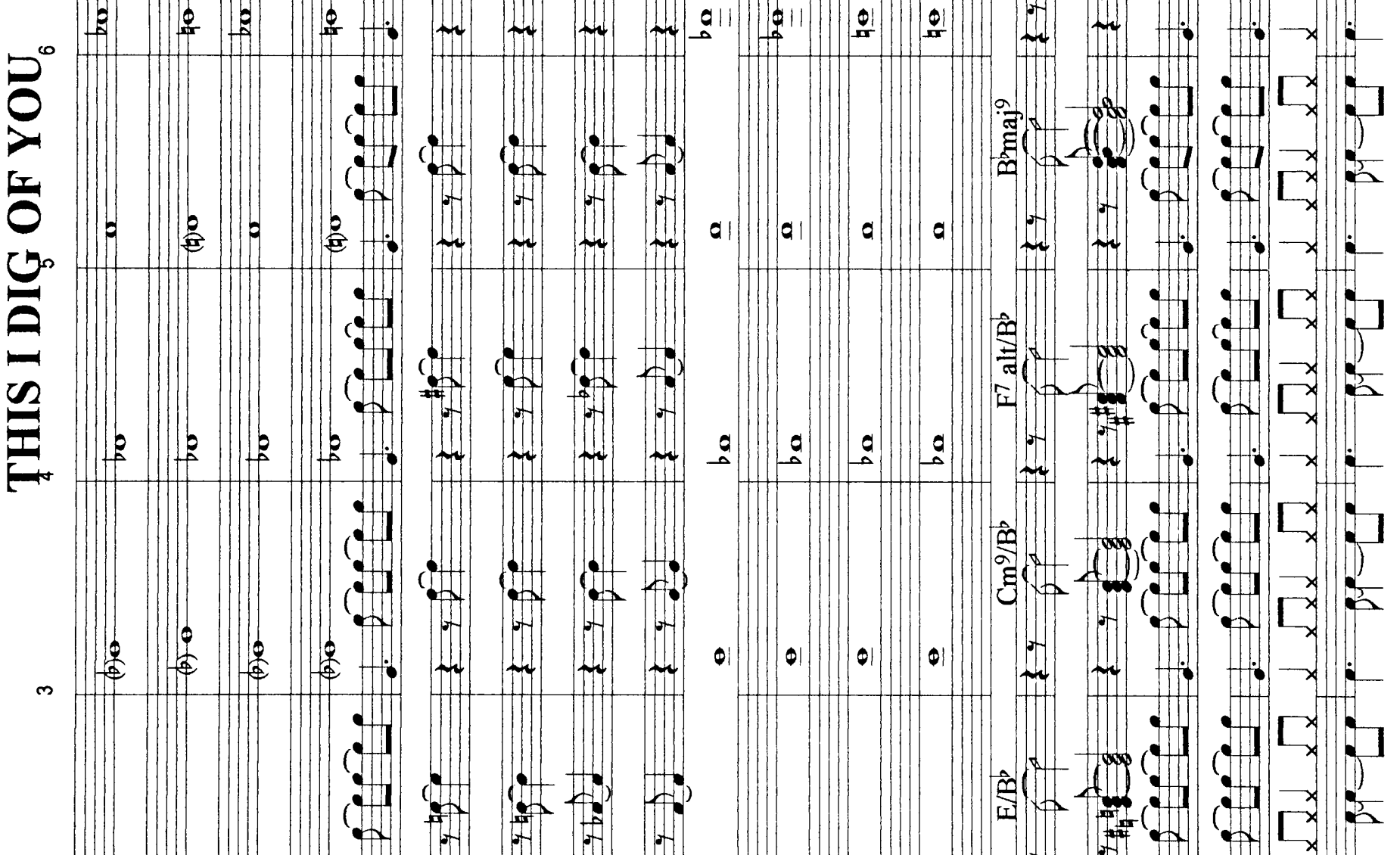

青) (1)

|

(a)

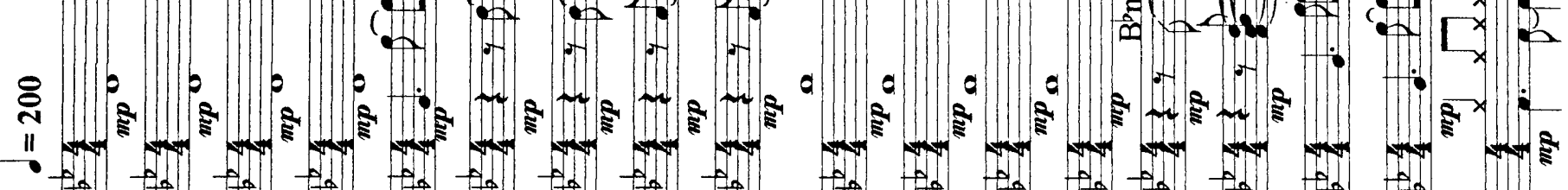

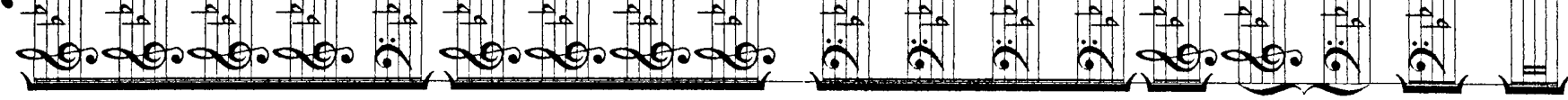

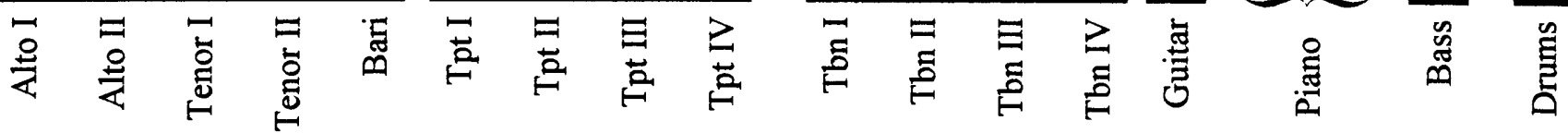




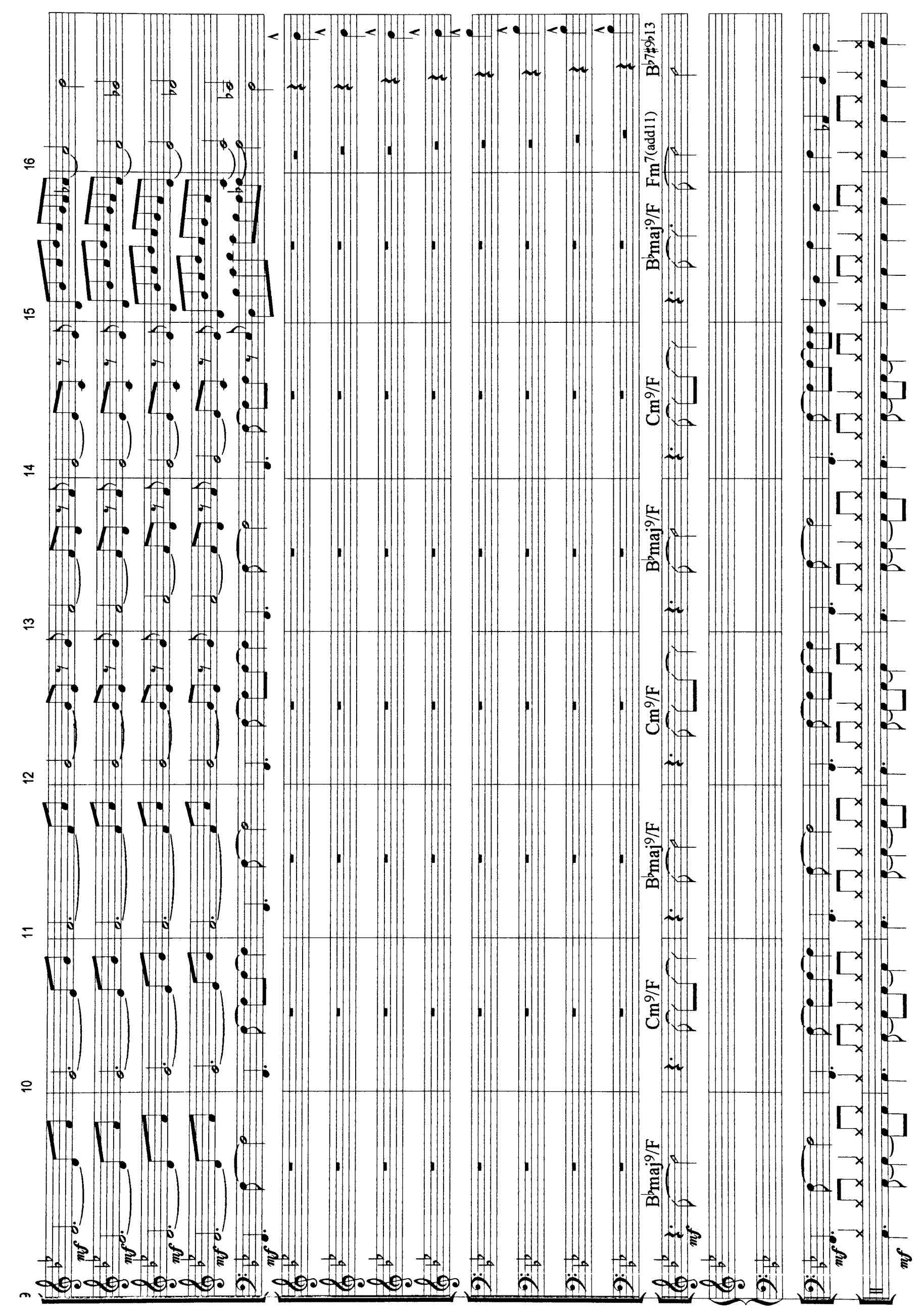


$\because \div \div \div$

H:

$\therefore: 10 \div: 1$

$\div$ :

1: $\because \because: 3$

\%

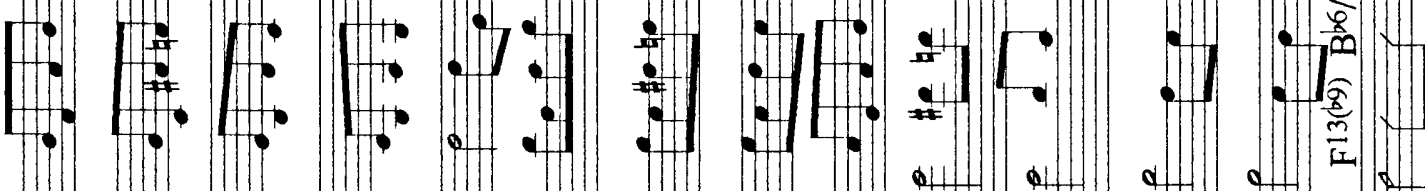

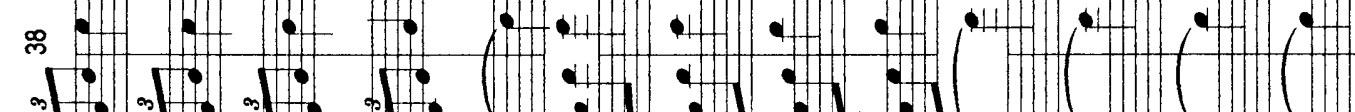

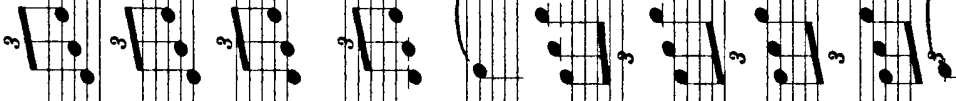

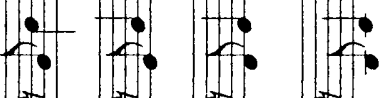

$2 \div \div=$

๓)

$\because 1: 1: \div: 0^{\circ}+\cdots+\cdots$

1:

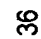
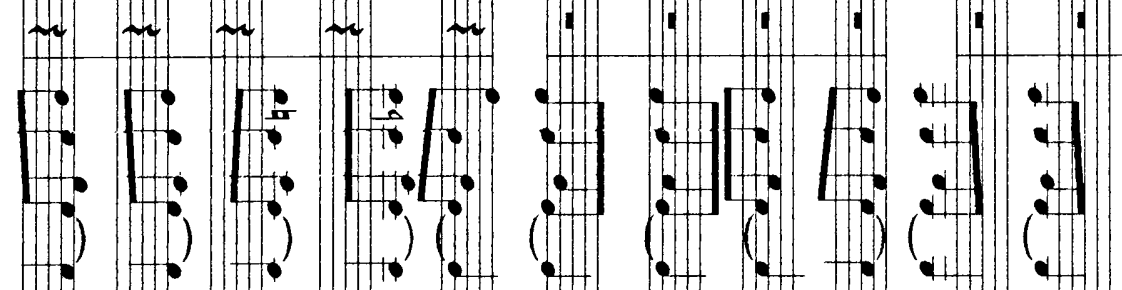

$\vdots::^{2}$

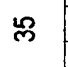

\begin{abstract}
$1+10$
\end{abstract}
(1)

- ‥

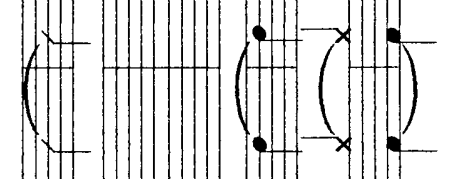

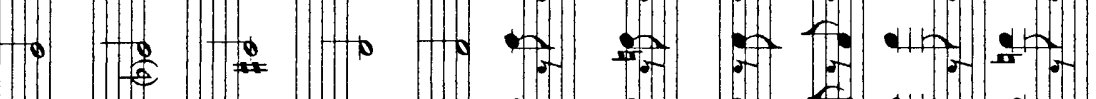

a) $2=22^{2}$

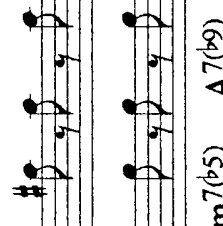

形
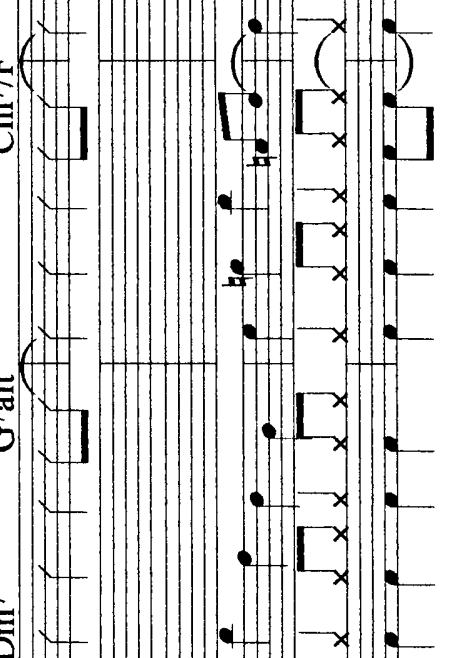

$4-x$

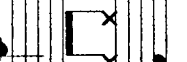

.

$1+x$

$4+x$

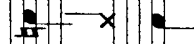

$-x_{x}^{x}$

f $x$

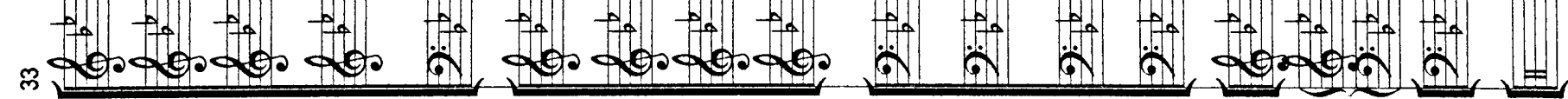




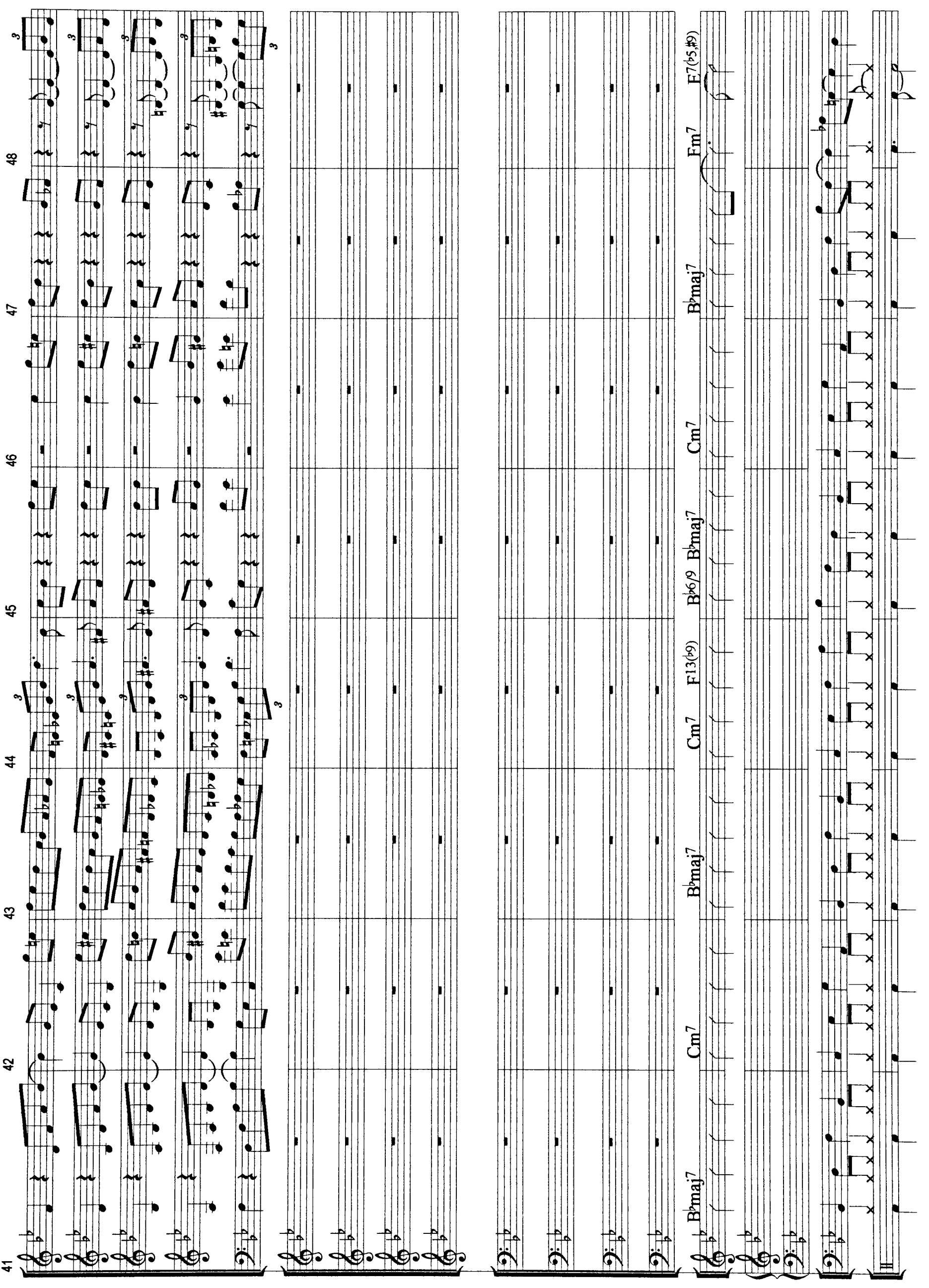




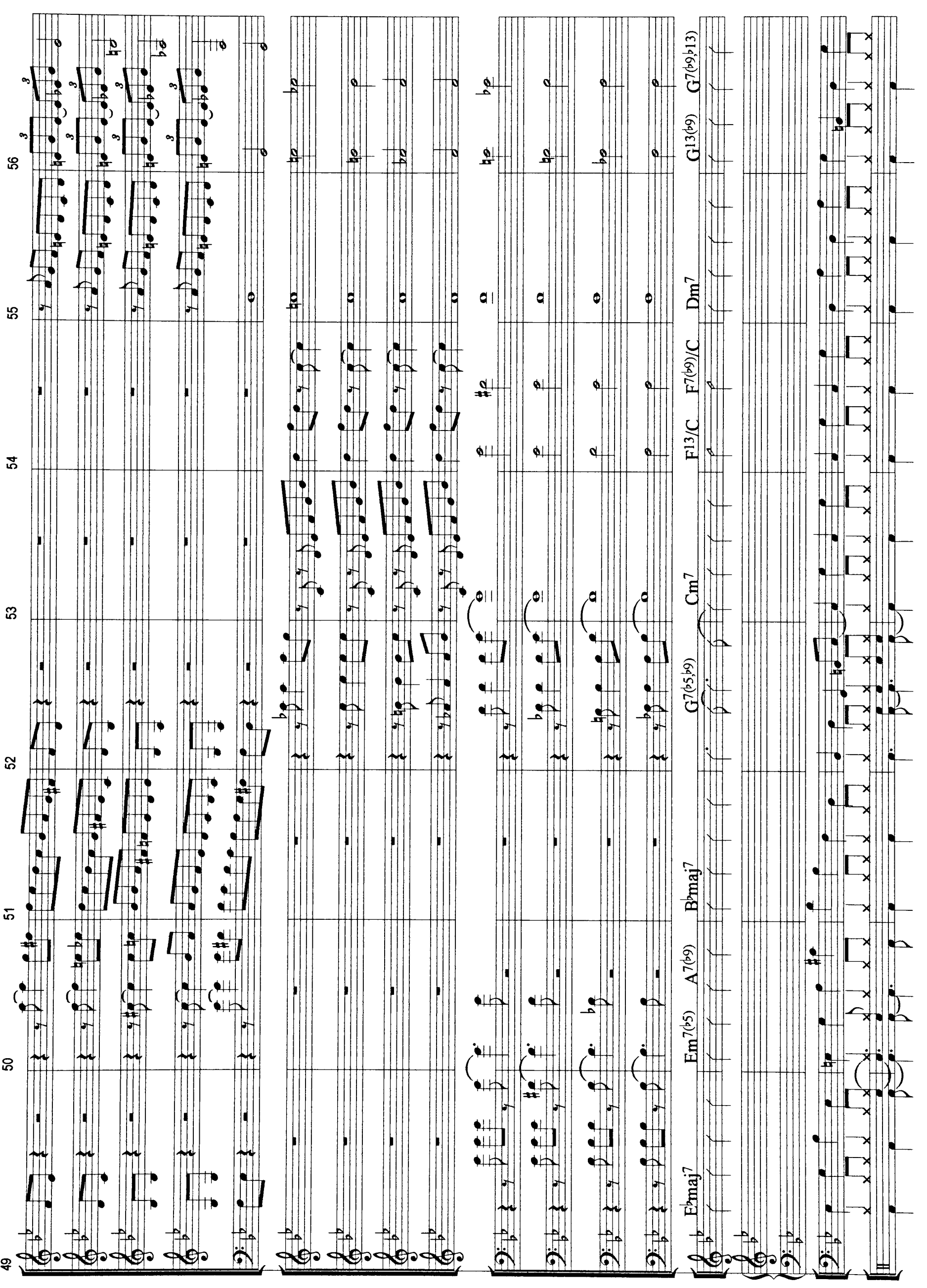




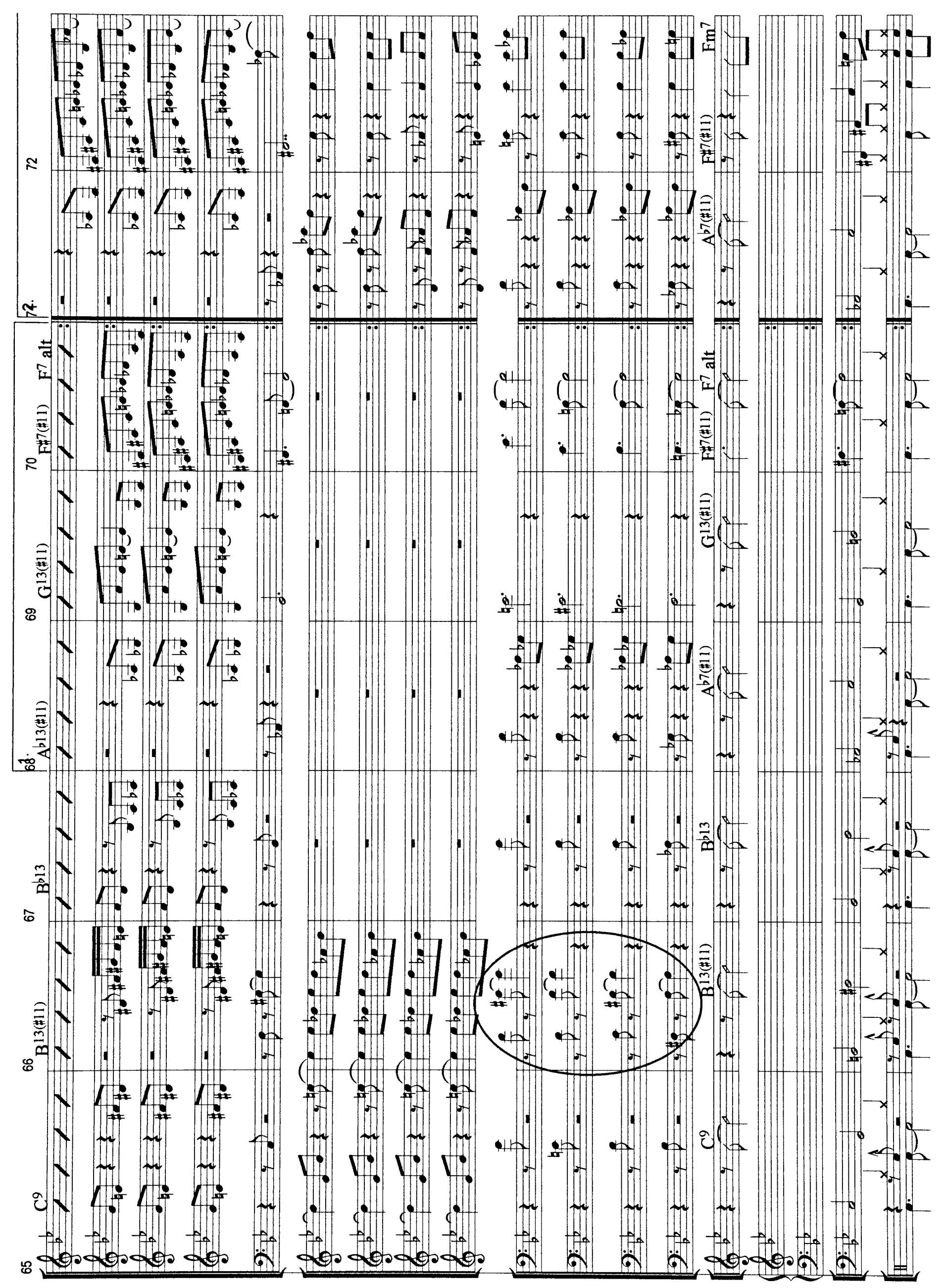




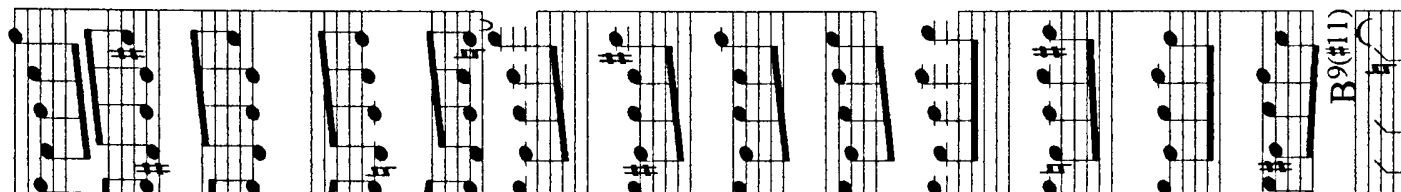

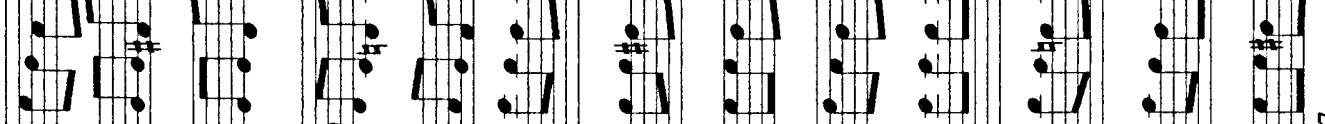

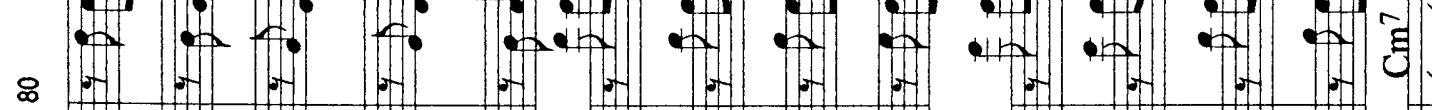

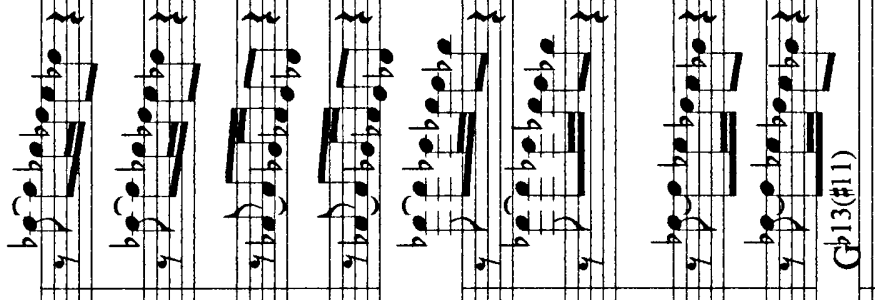

$\therefore: \div: \div: \because$

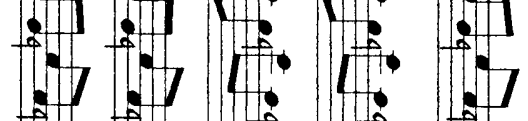

青期

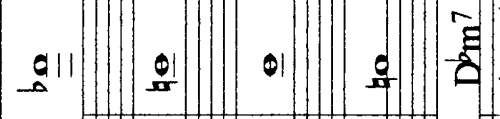

$4 x-2$

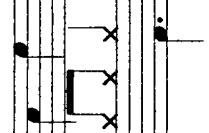

$+x-x$

- $x$

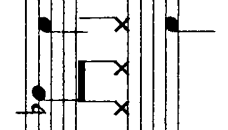

$\therefore \times$

: $x$

- $x$

$-x-$

- $1 x$

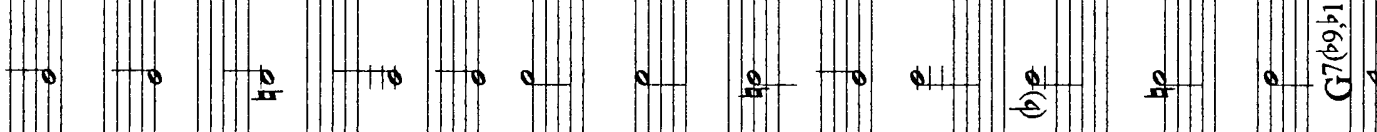

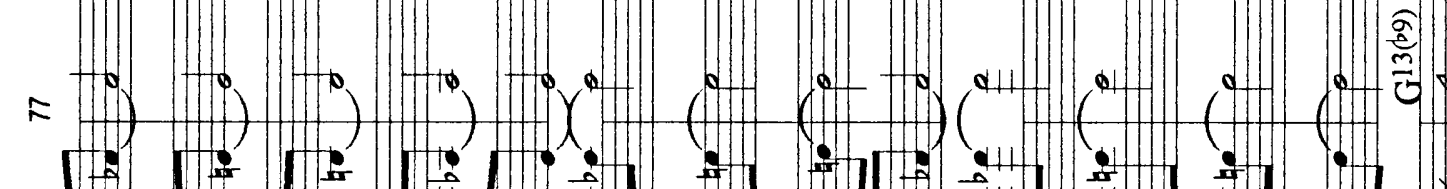

an $x$

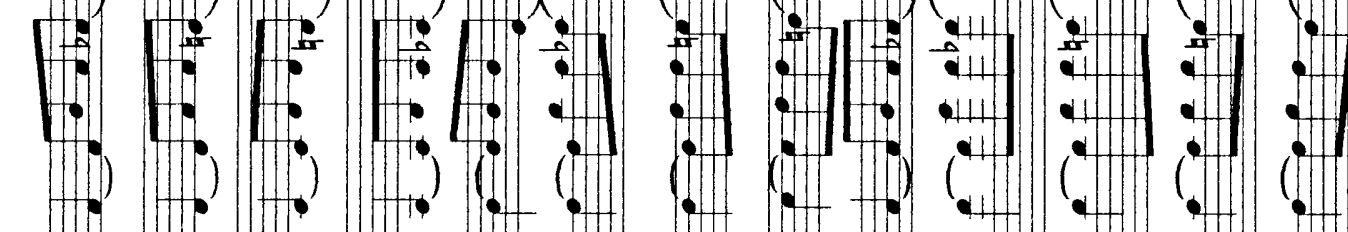

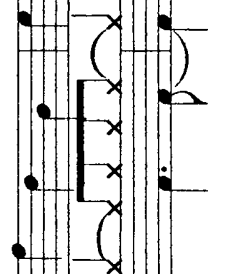

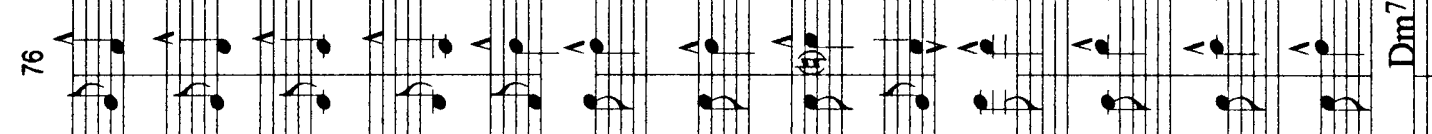

A.

f.

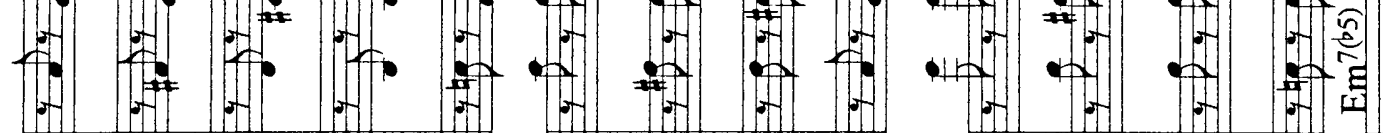

11 f f

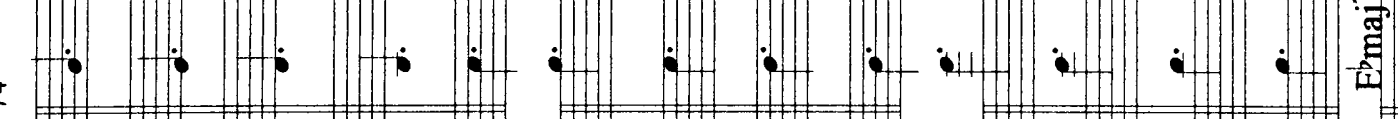

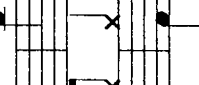

- ${ }_{x}^{x}$

2. $1 x$

$+5$

- $1 x$

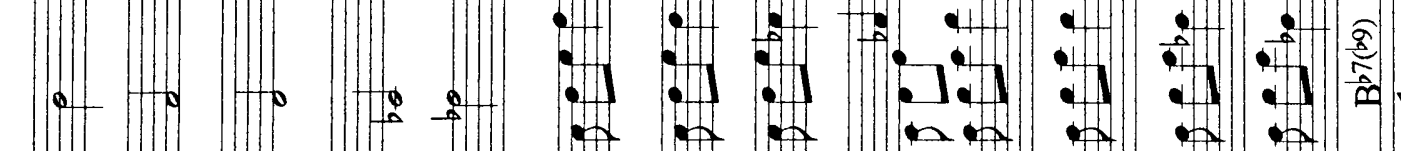

- $x$

- $\begin{aligned} & x \\ & x\end{aligned}$

$-x$

$\left[\begin{array}{ll}x \\ x\end{array}\right.$

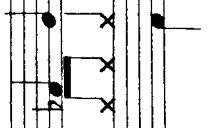

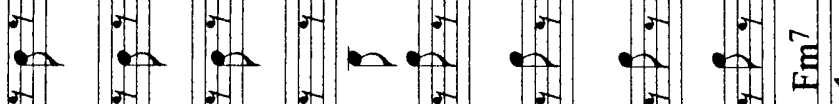

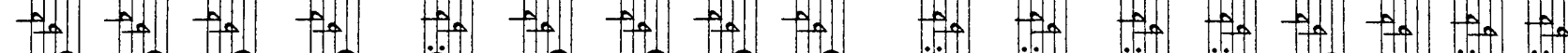

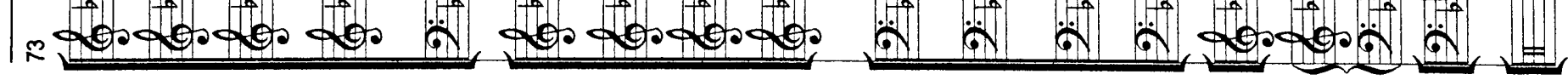




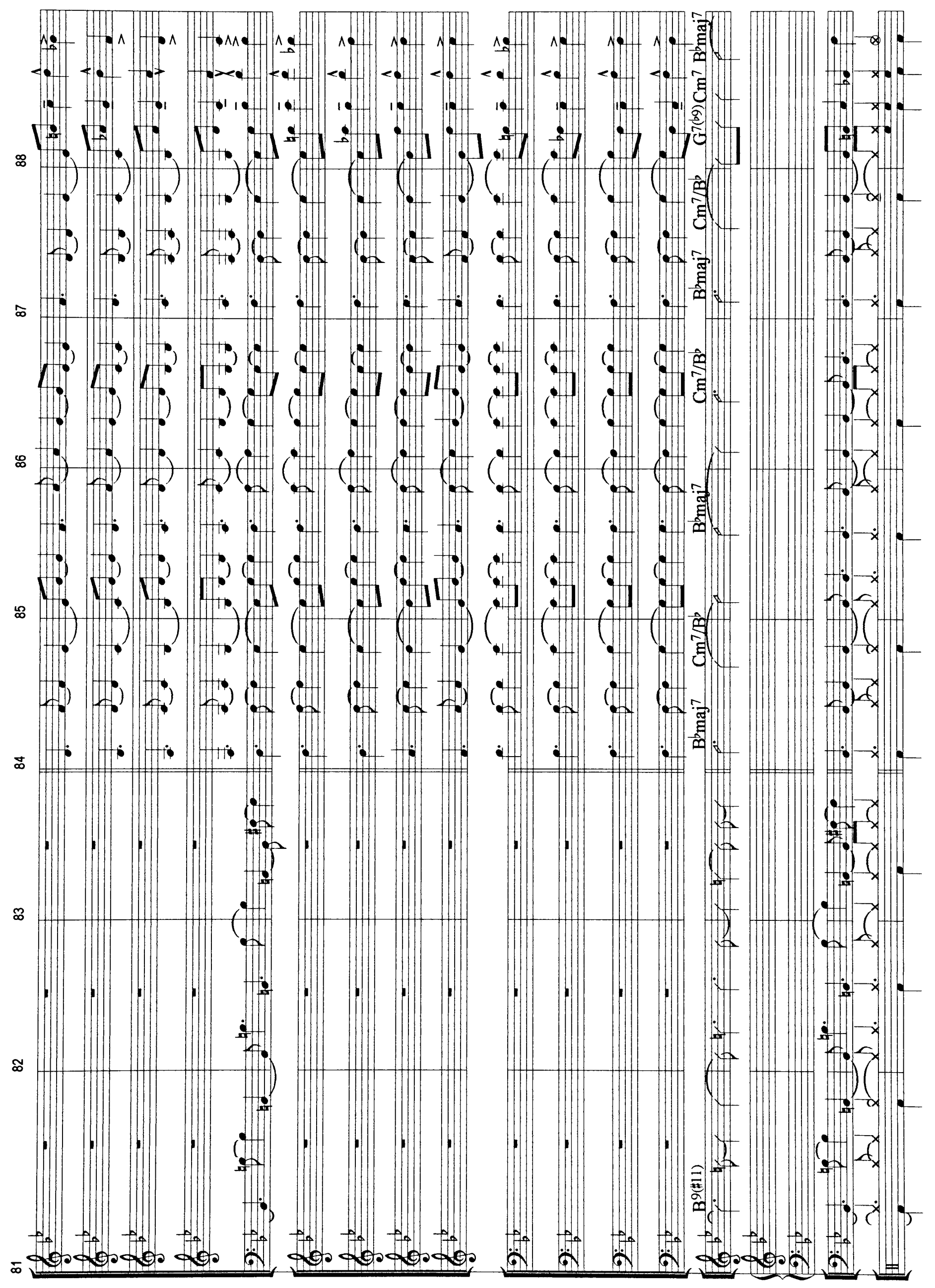


c

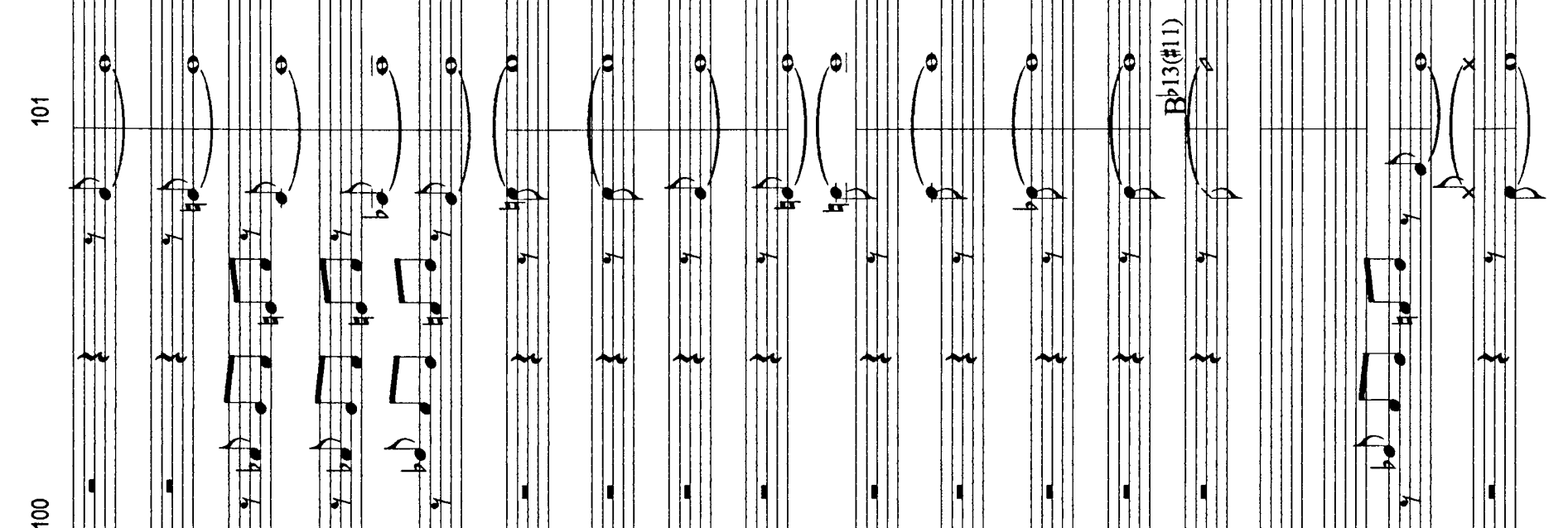

ㅇ

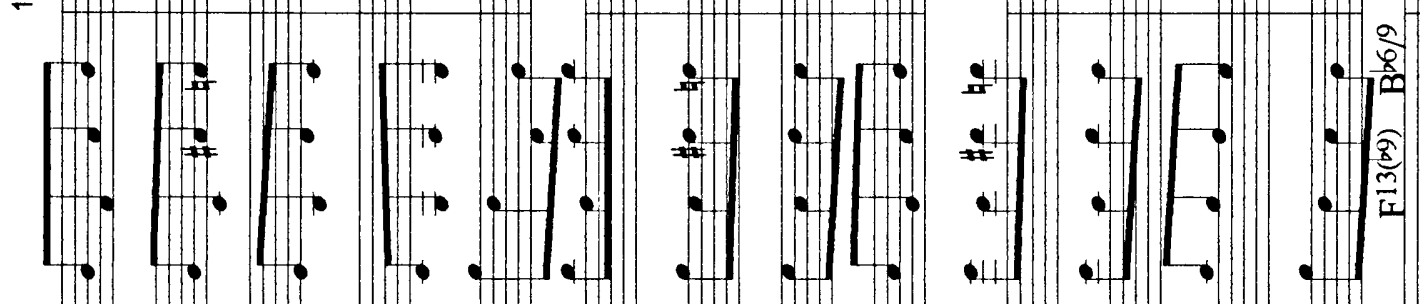

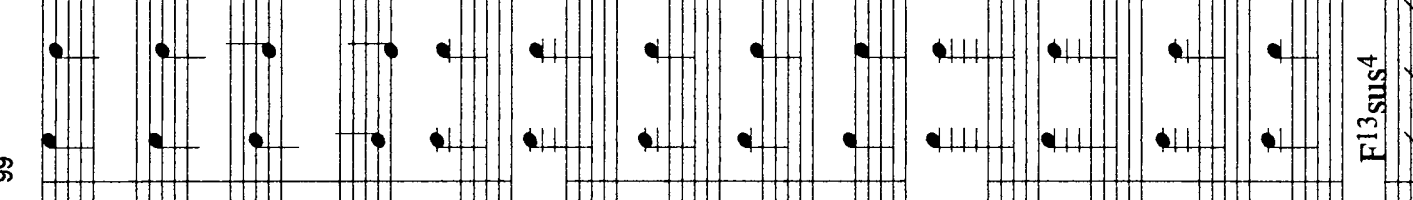

D.

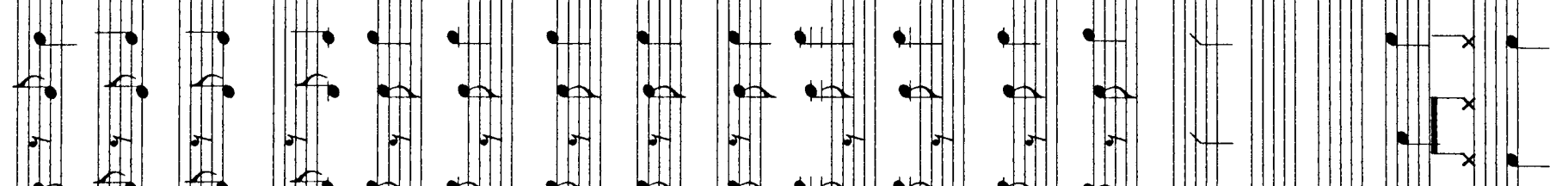

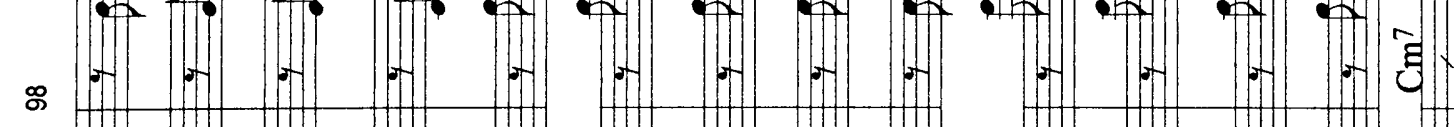

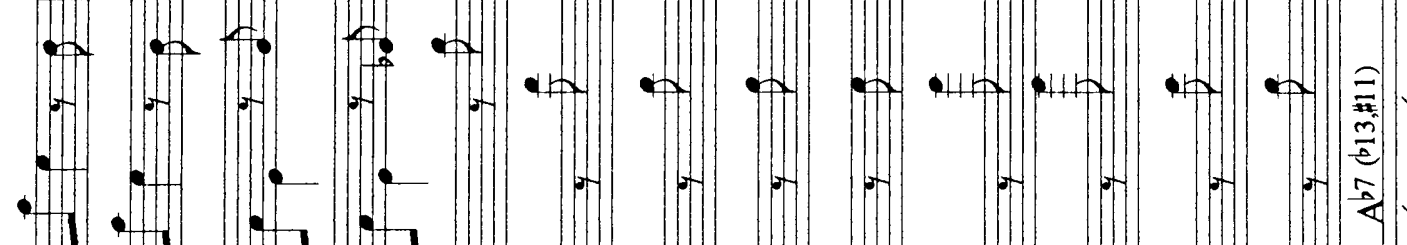

$\therefore-1 ; \div-1$

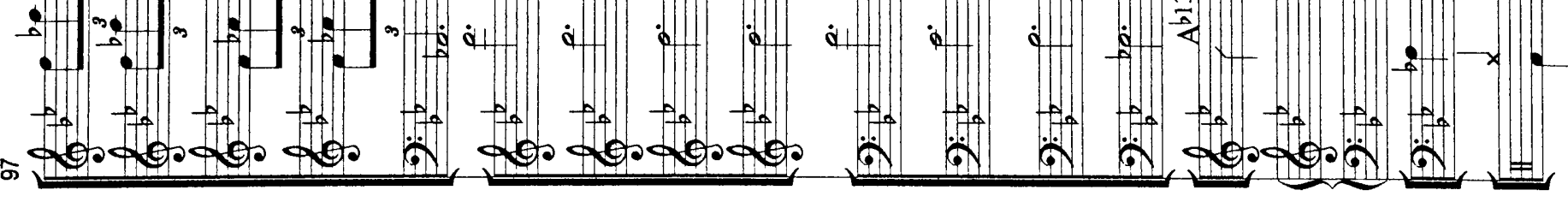




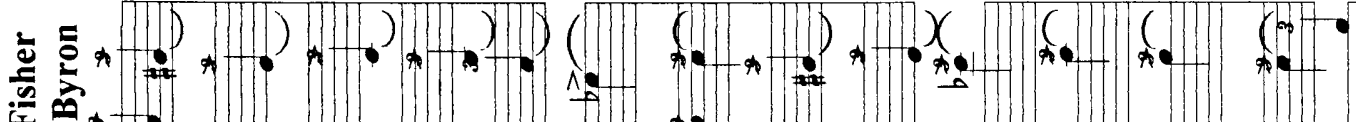

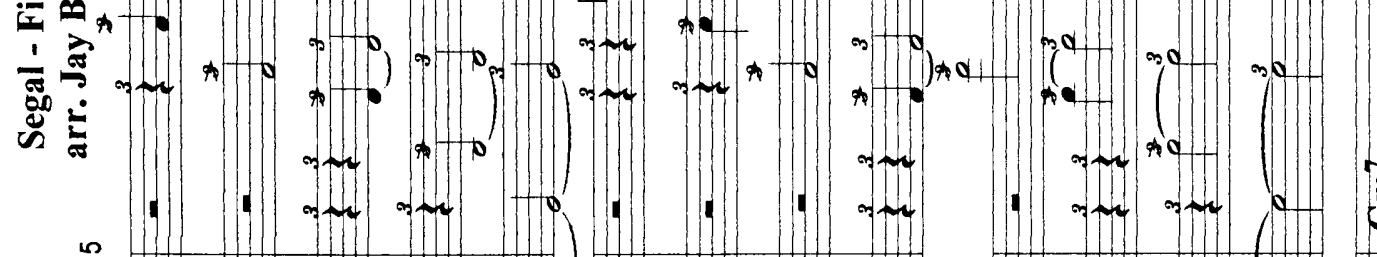

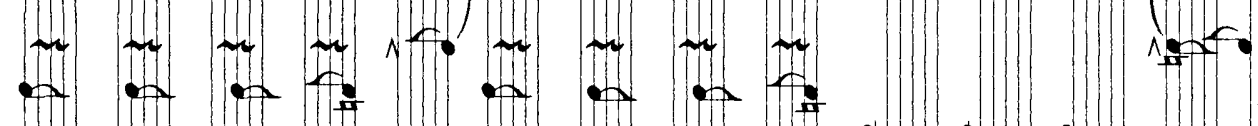

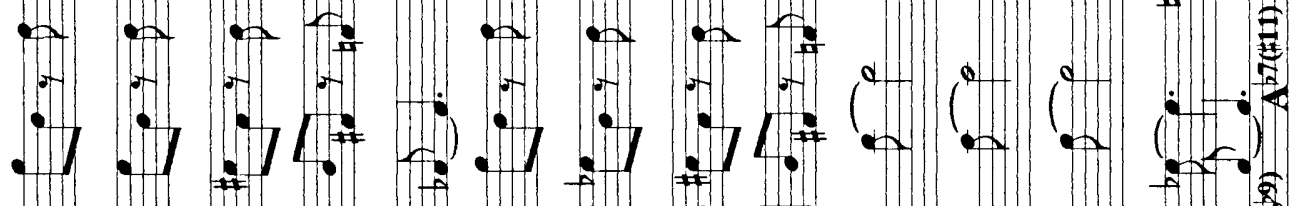

$x i$

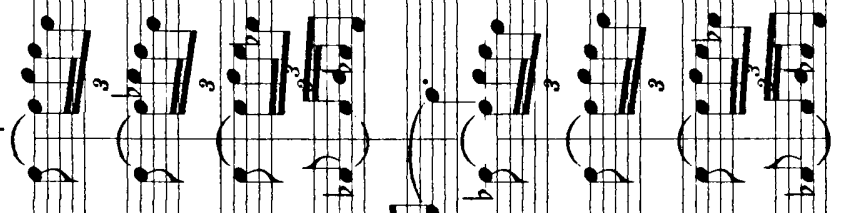

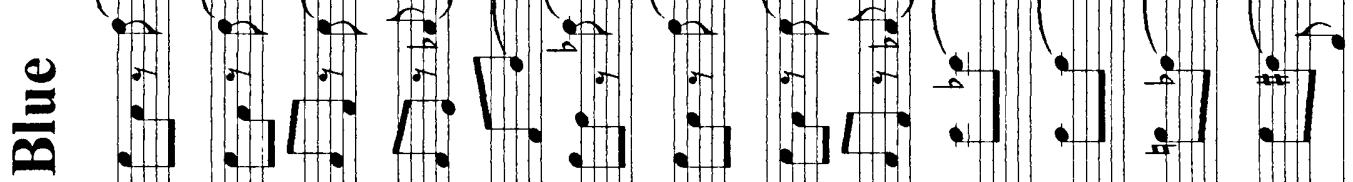

$\therefore: \therefore: \therefore:$

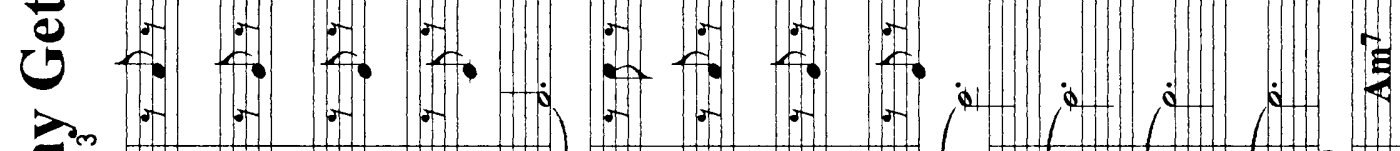

. : : : : : : : : : : : :

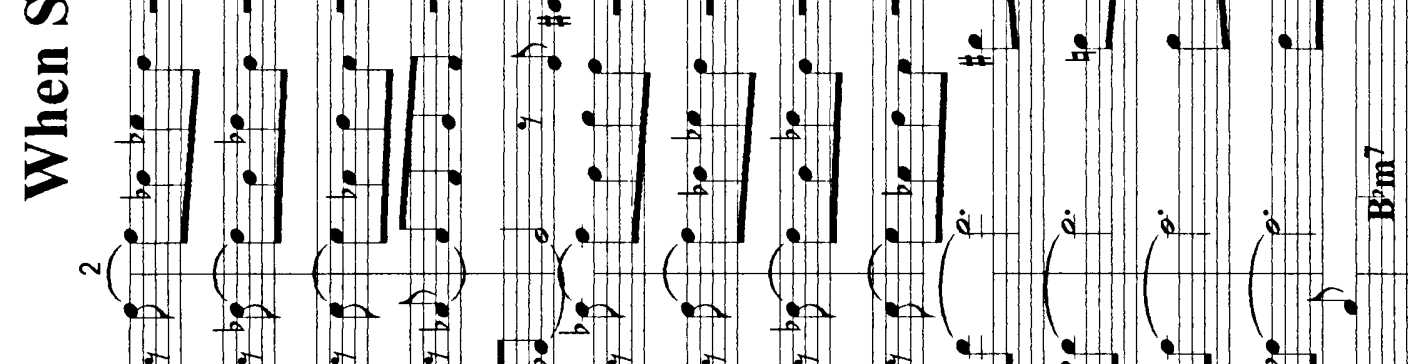

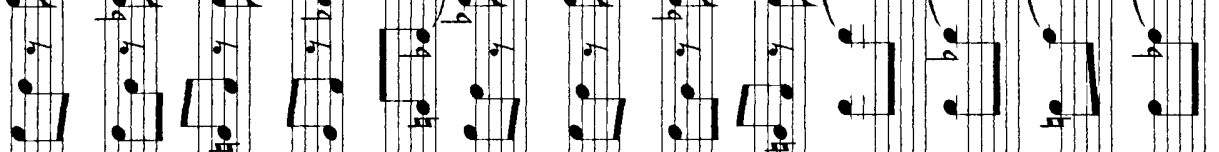

$\because: \div: \because: \because \div$

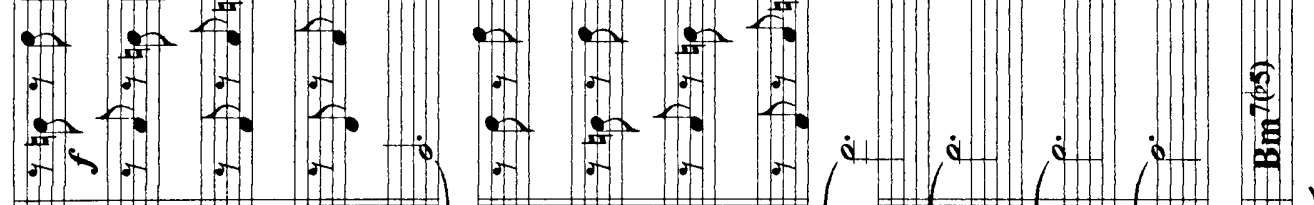

8
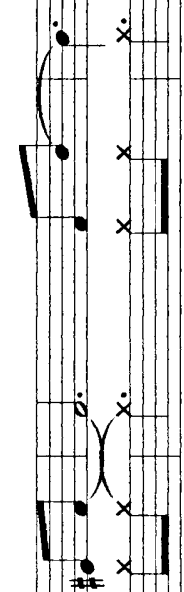

$5 \times$

$+1$.

$10 x+1$

1.

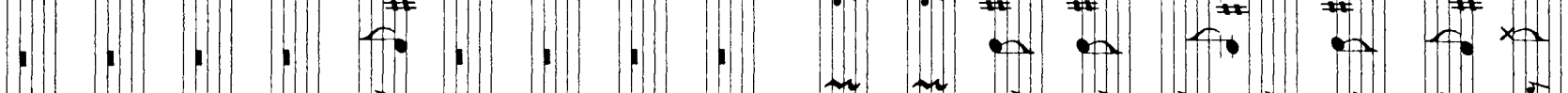

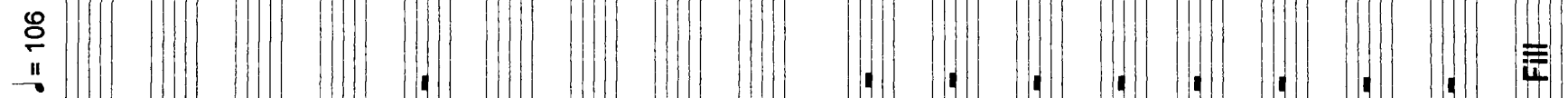

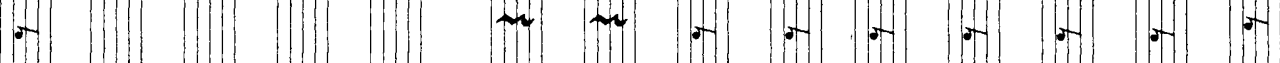

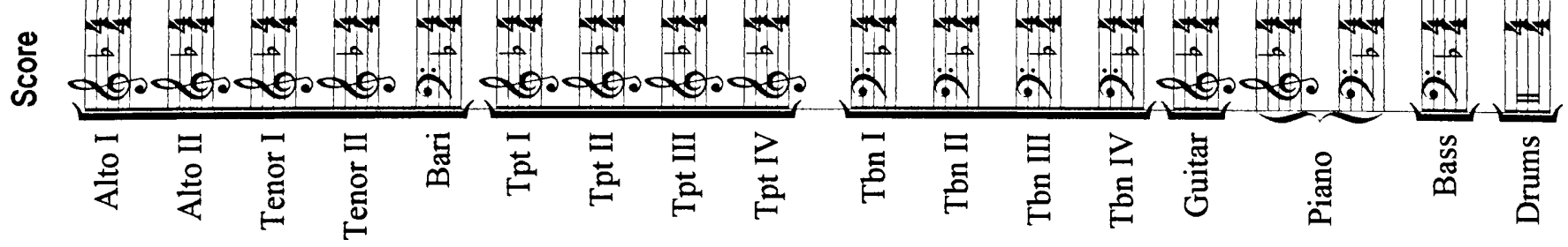




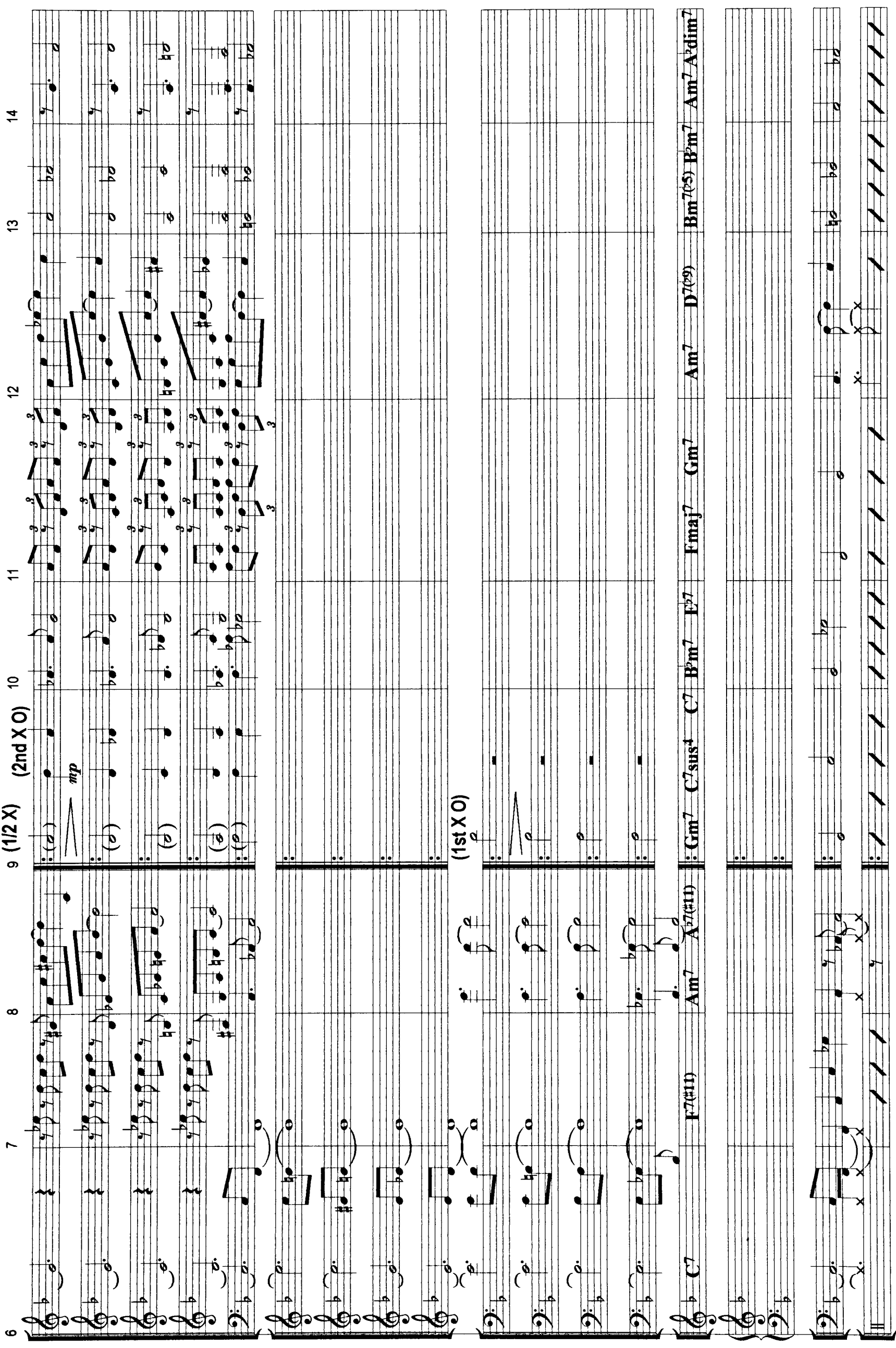




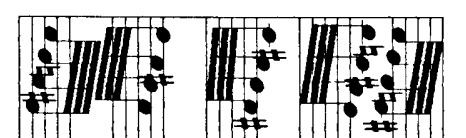

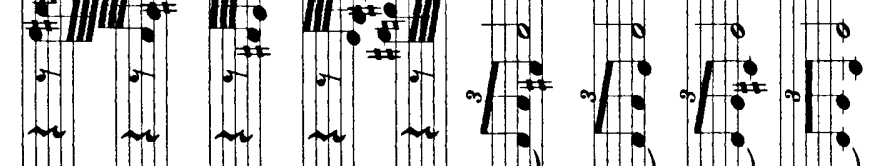

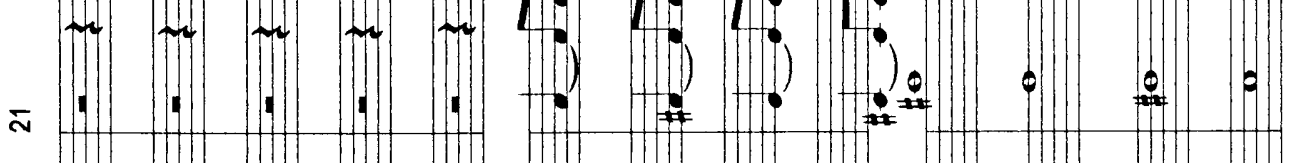

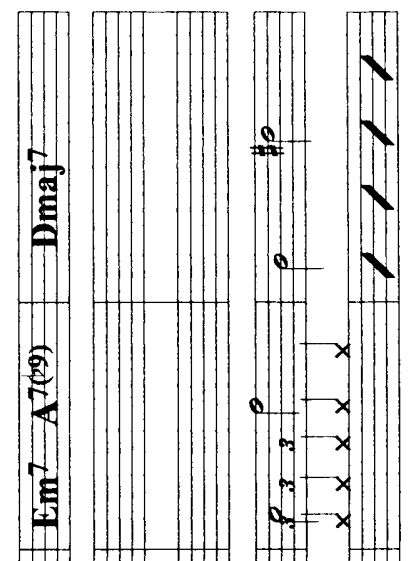

요

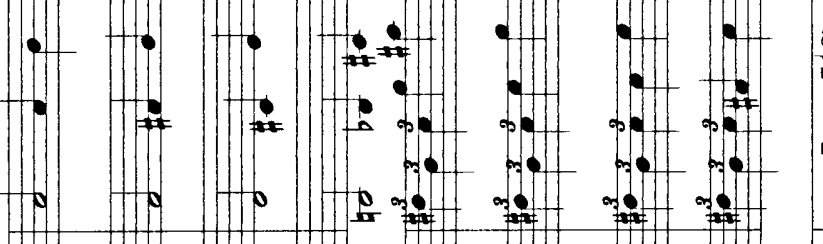

를

$\gamma$

2.

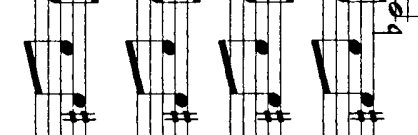

웅

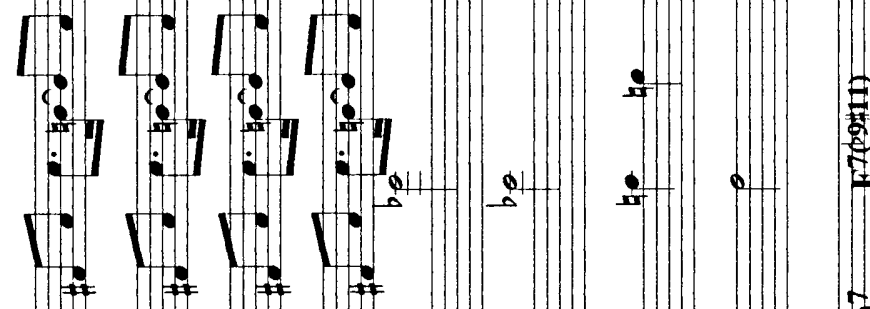

:

: $x$

部

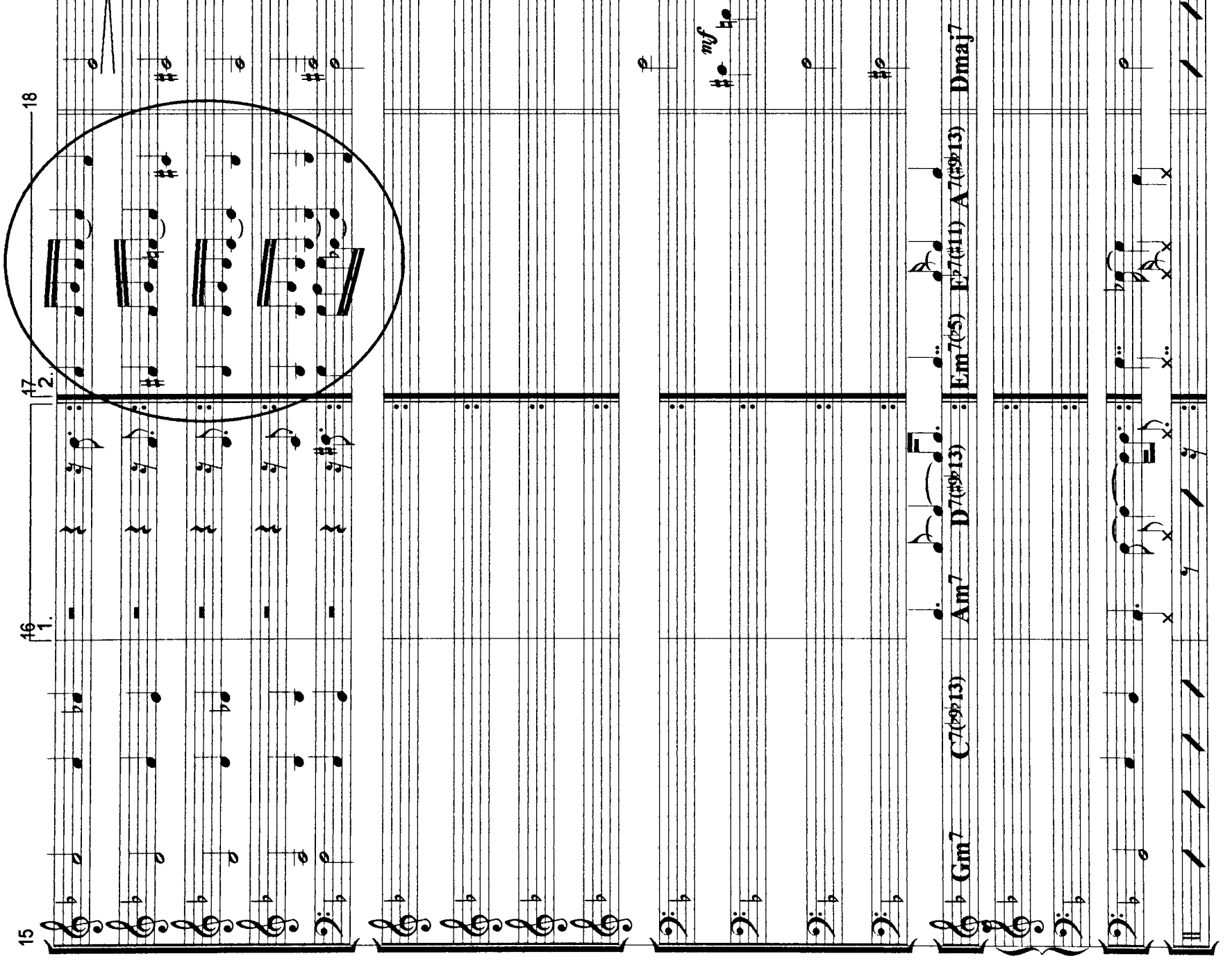


州

-

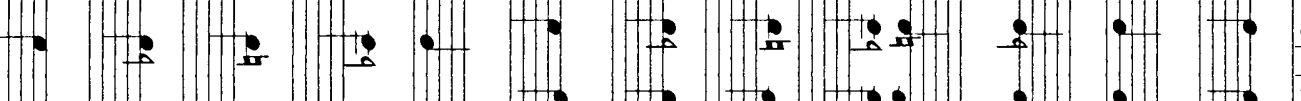

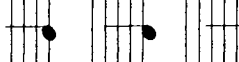

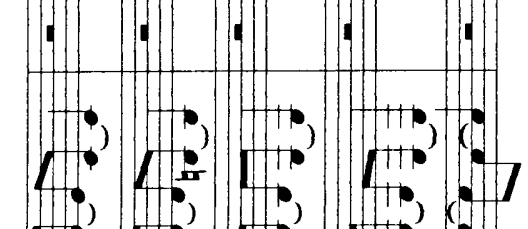

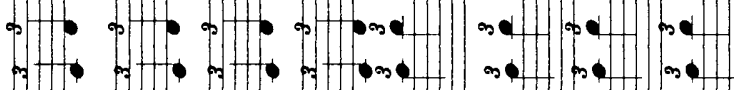

䓵

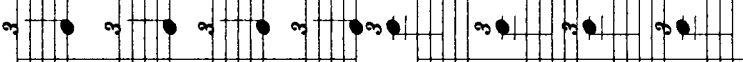

ह

昜 $=$

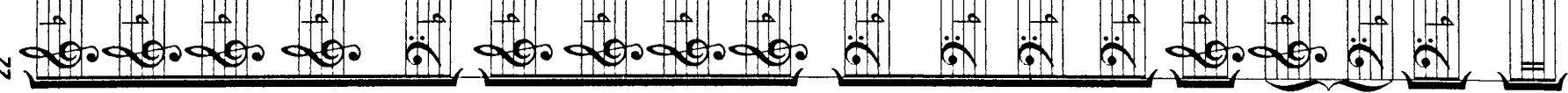


$\stackrel{\circ}{\circ}$ 앙

- 10 110 0.0.

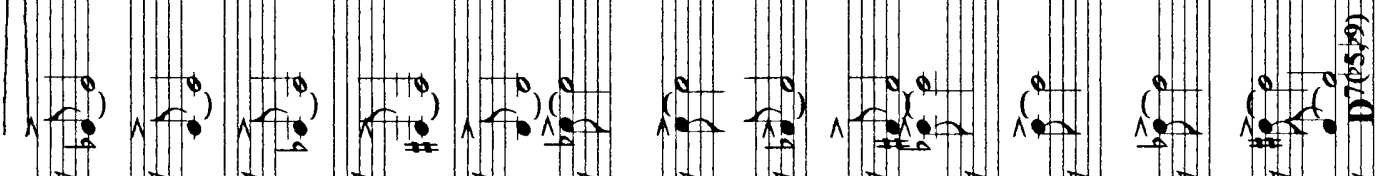

$\log _{x}$

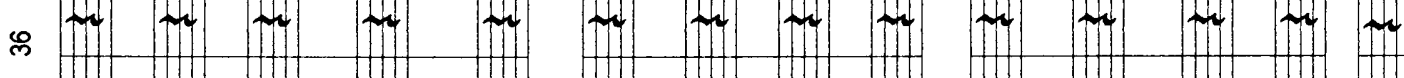

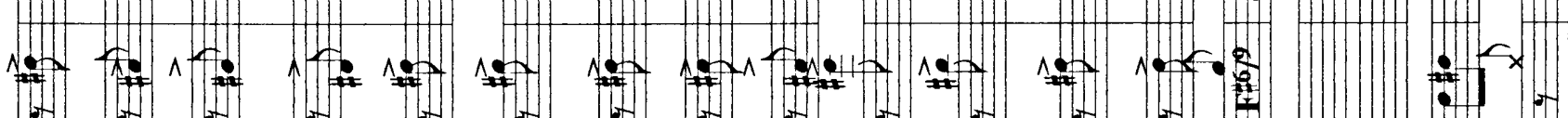

$\left.\infty \prod_{-\infty}^{\infty}\right)(+\infty$

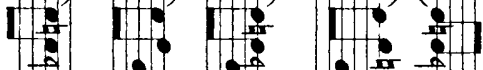

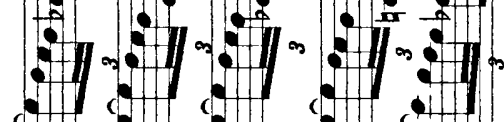

$\therefore 1 \rightarrow 1 \rightarrow 1 \rightarrow 1$

पू की की की की

$\frac{\bar{x}}{\overline{2}}\|\|_{0}\|\|$

ह
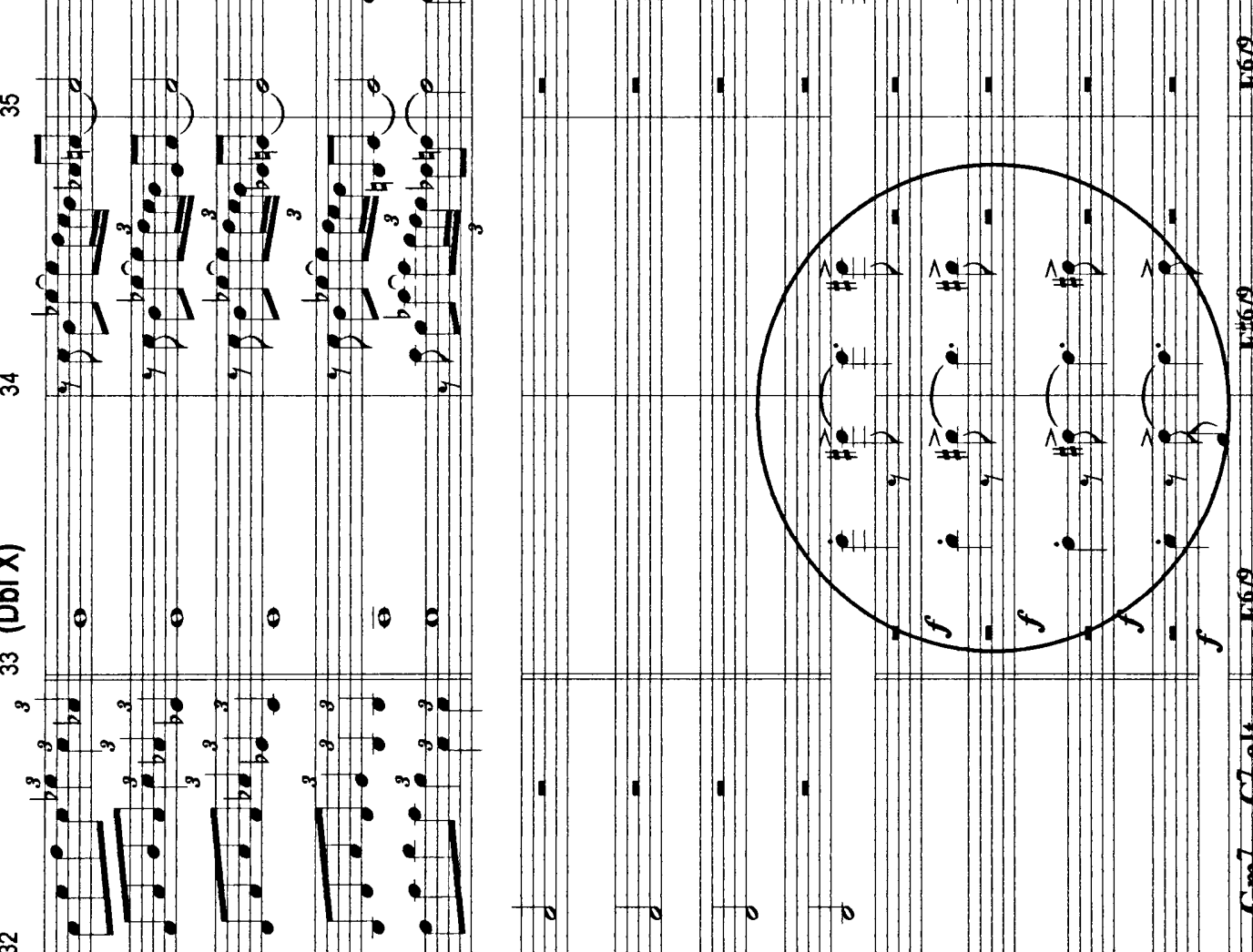

5
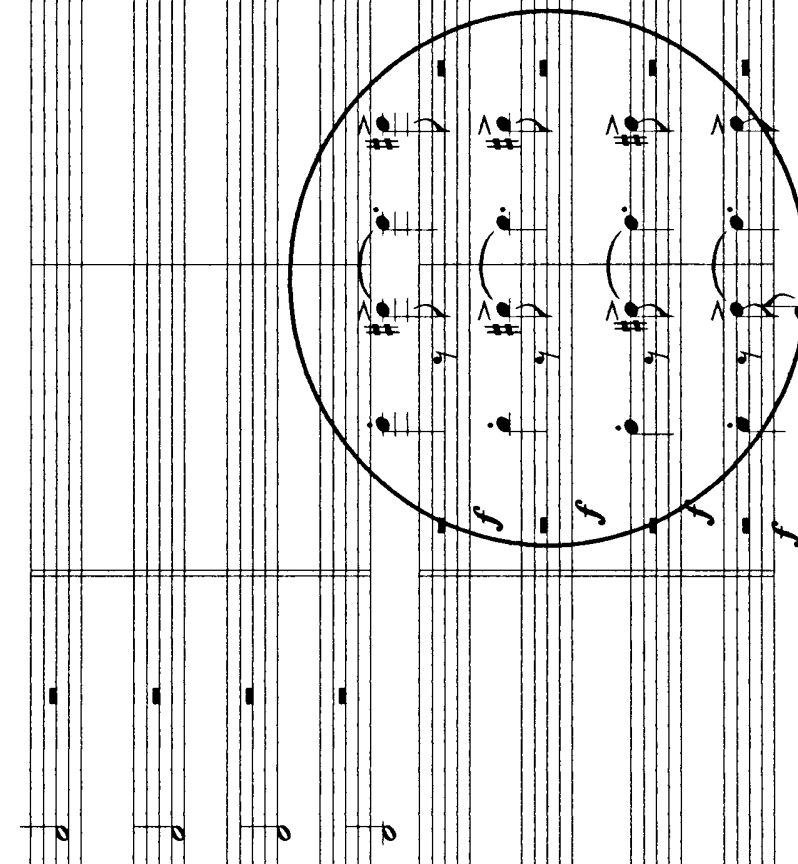

$\sqrt{8}$

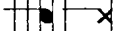

(ffo

- Hi H:

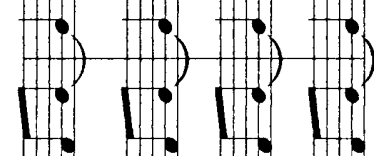

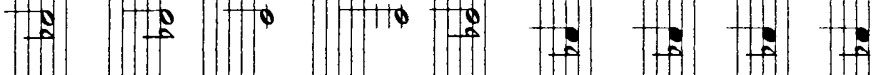

E

:

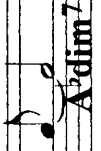

$: \frac{1}{4}$

正

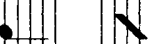

- N

$-1$

- $1-1$

$+$

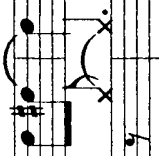




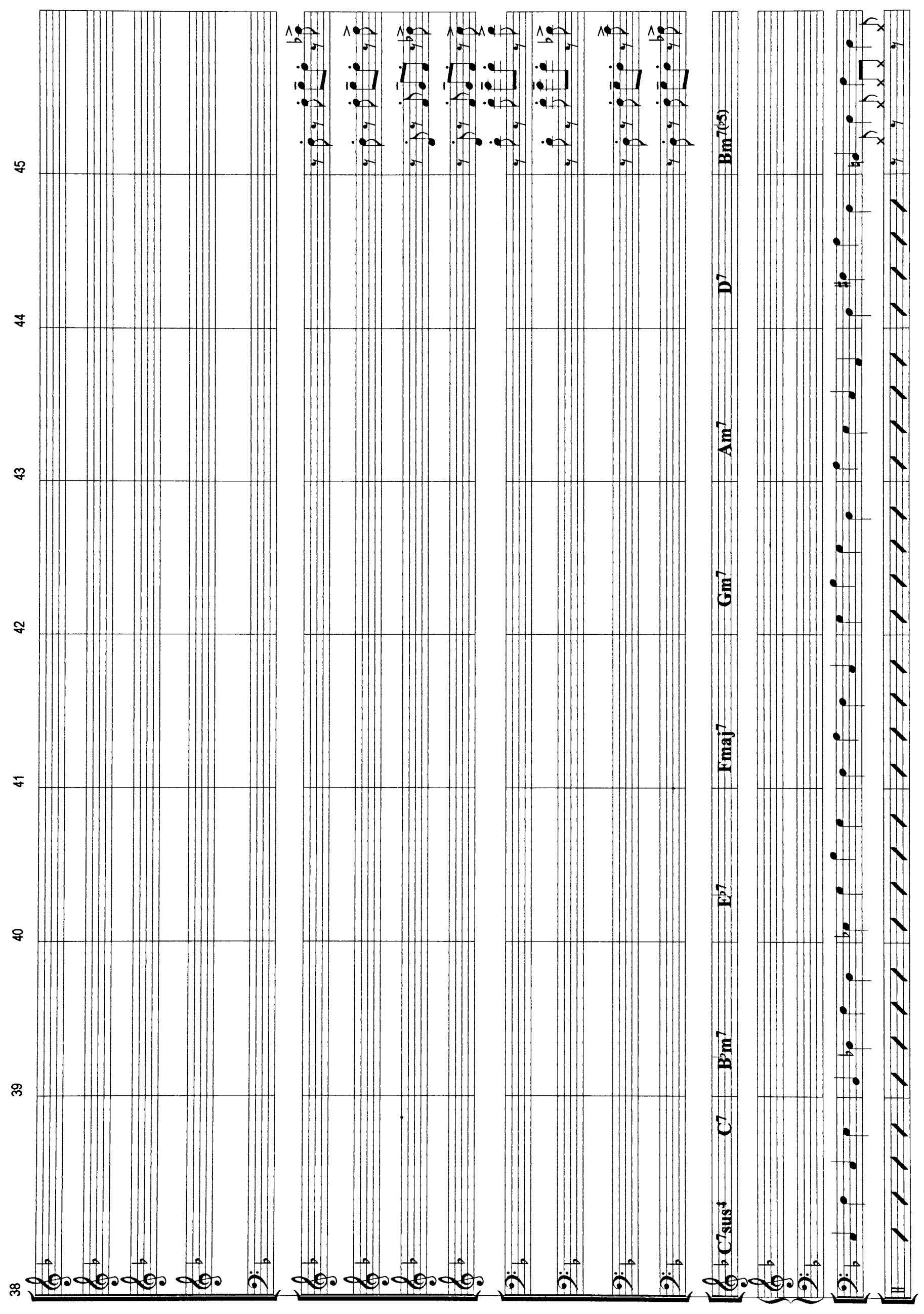




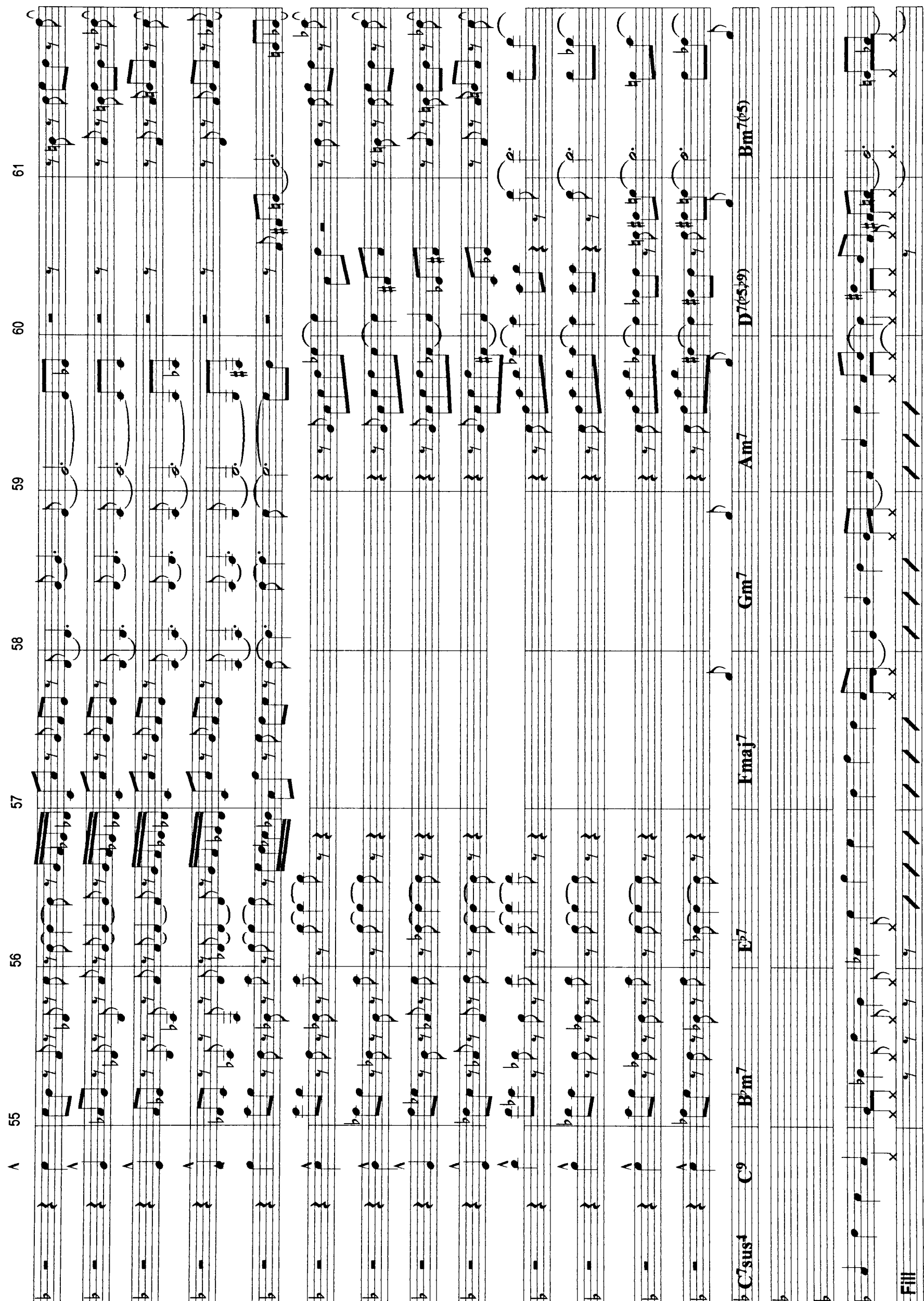

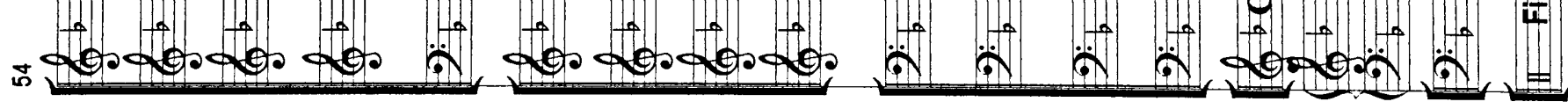




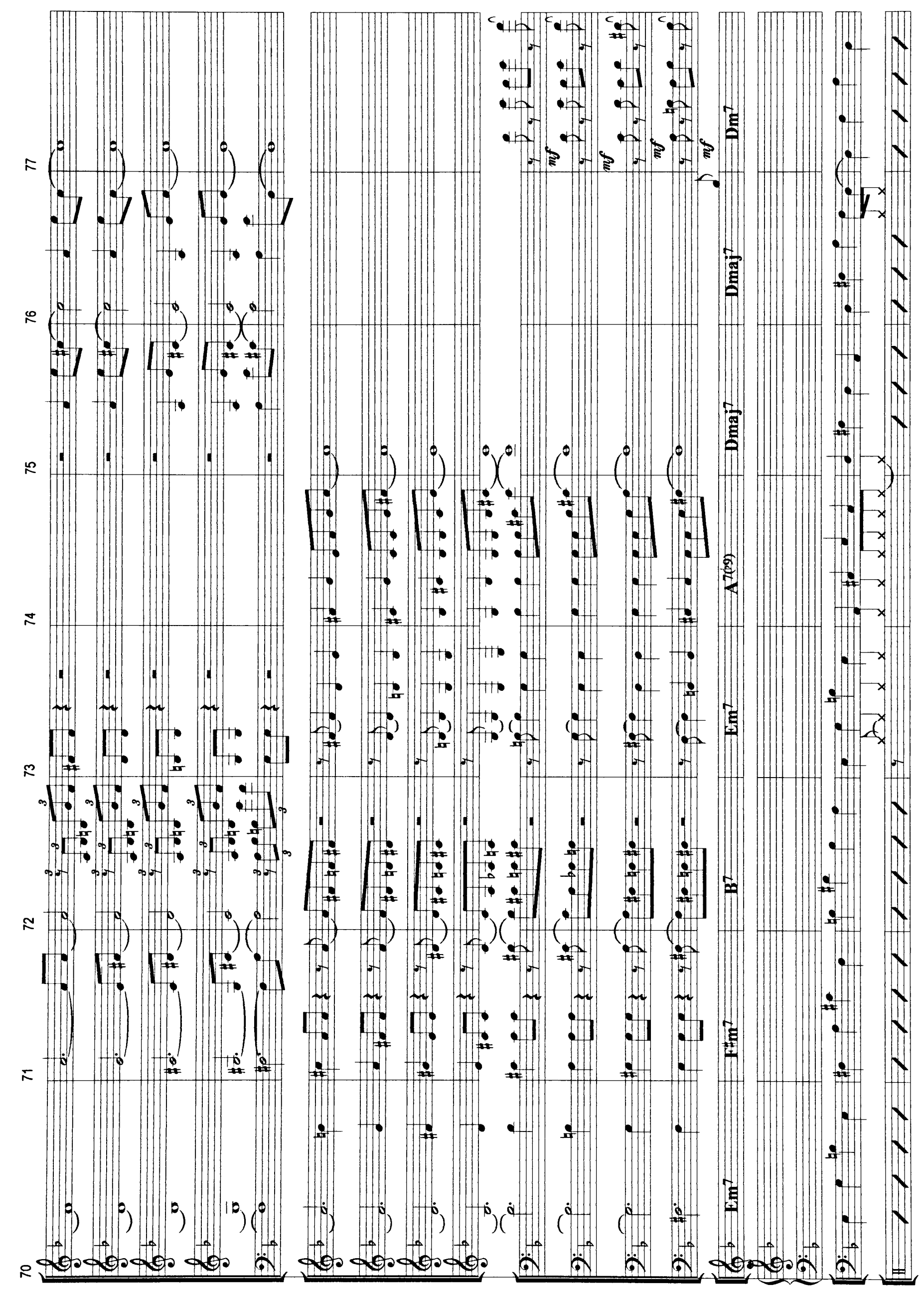


$\mathbb{1}: \mathbb{1}: \mathbb{E}: \mathbb{1}: \vdots$

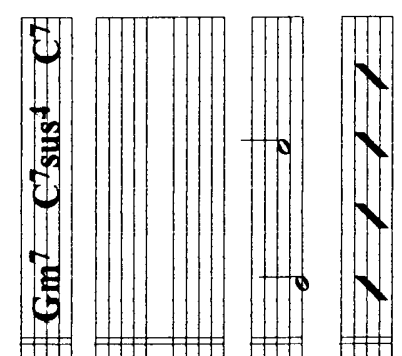

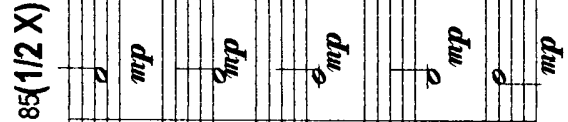

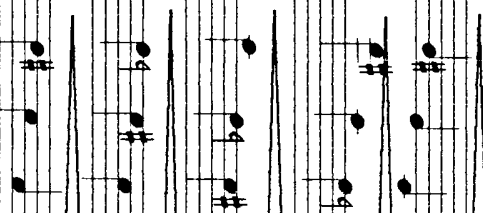

品
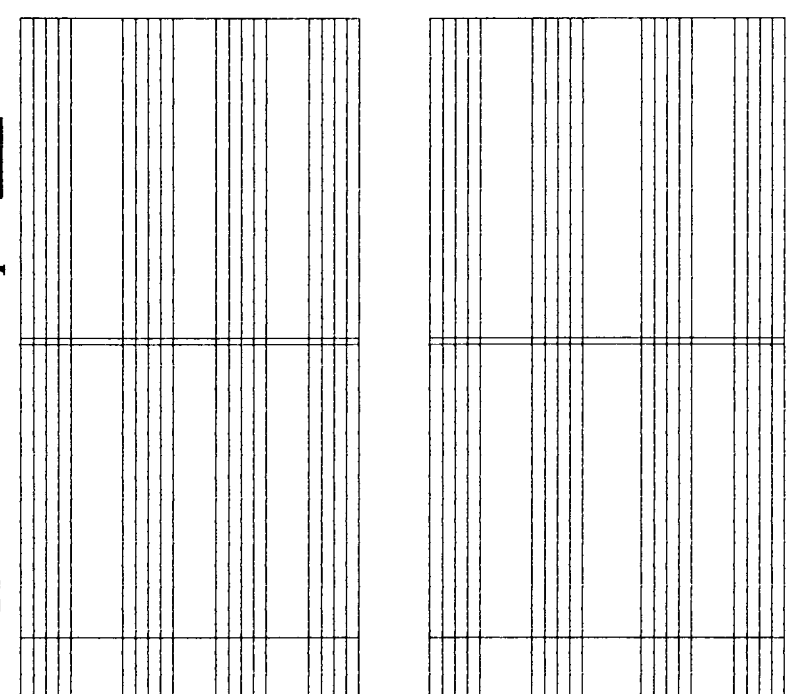

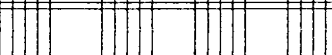

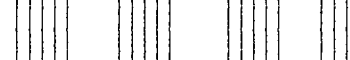

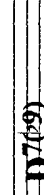

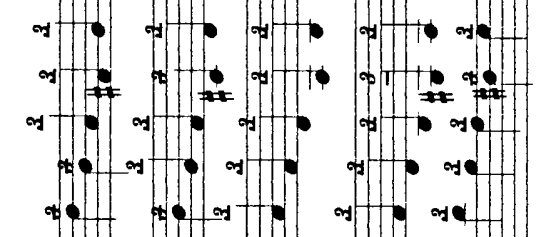

(1) (-)

.

$\operatorname{lox}_{x \rightarrow-1}$

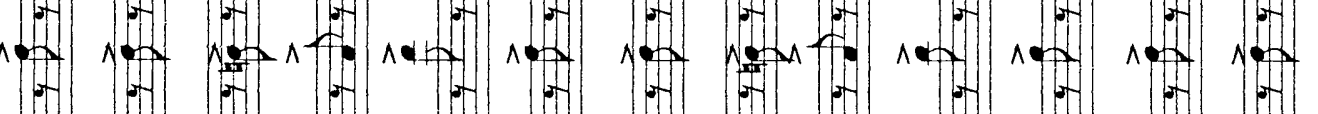

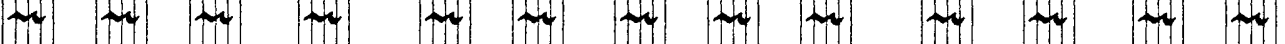

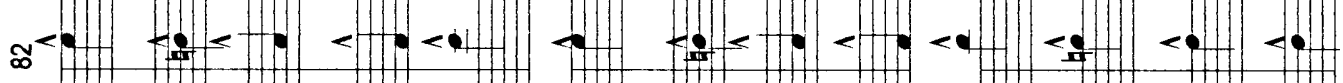

6

$1 .+1+10$

$1+1+1,10$

(1) - (A) $\wedge$ H

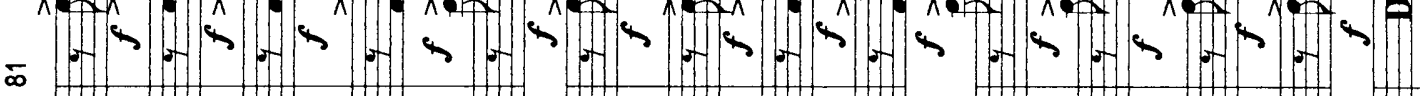
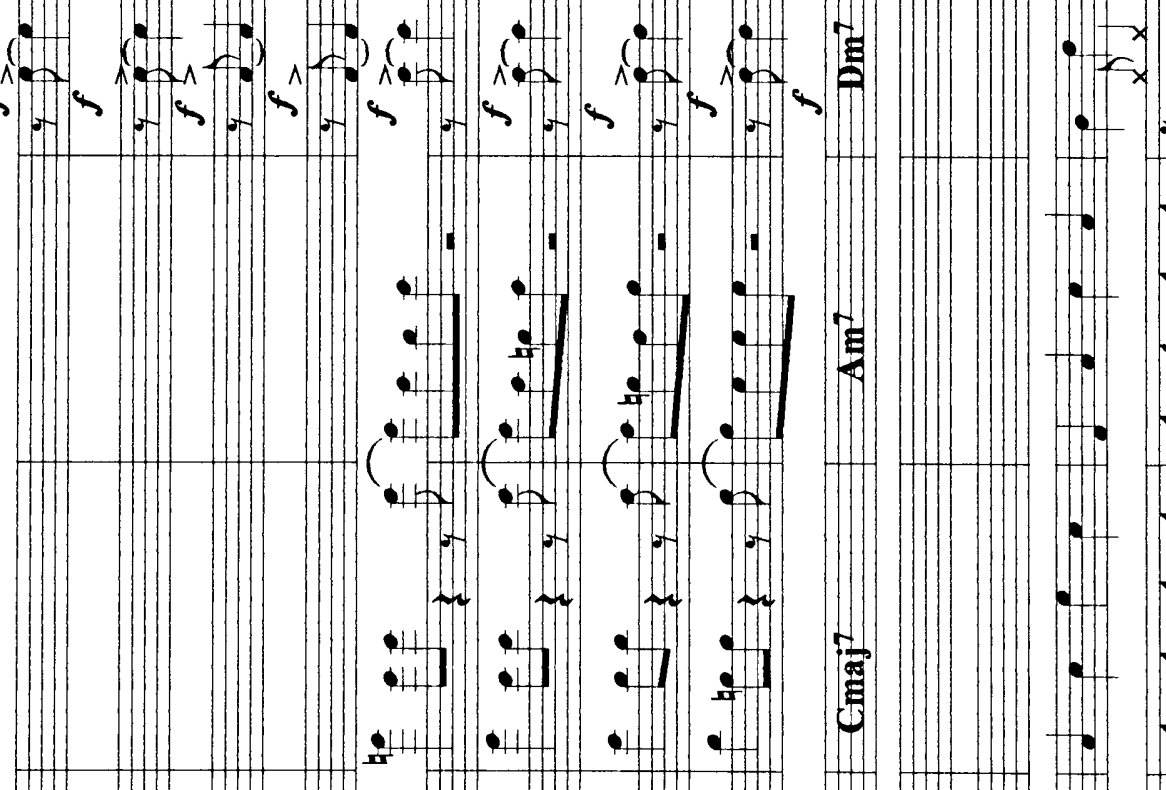

s

1.11) 11 -1)

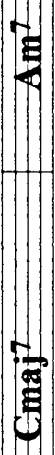

- $x$

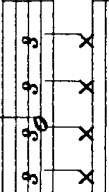

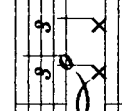

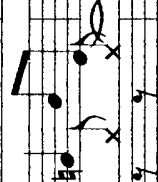

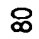

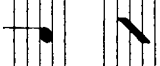

(1)

U

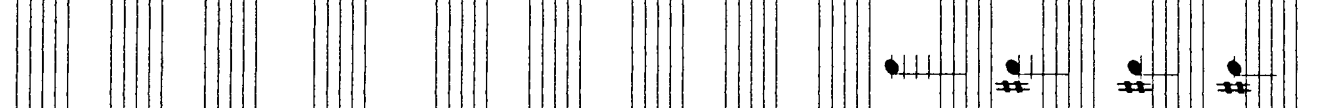
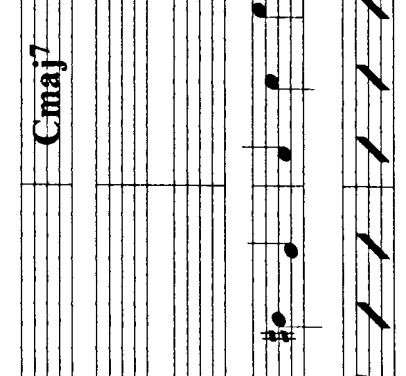

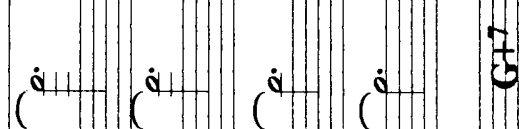

A

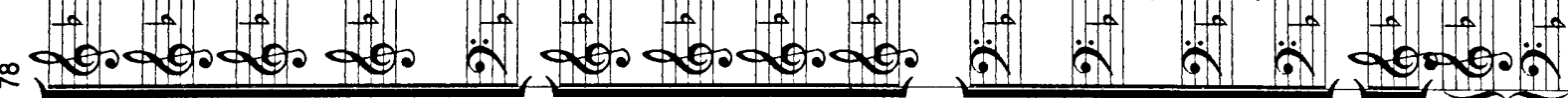




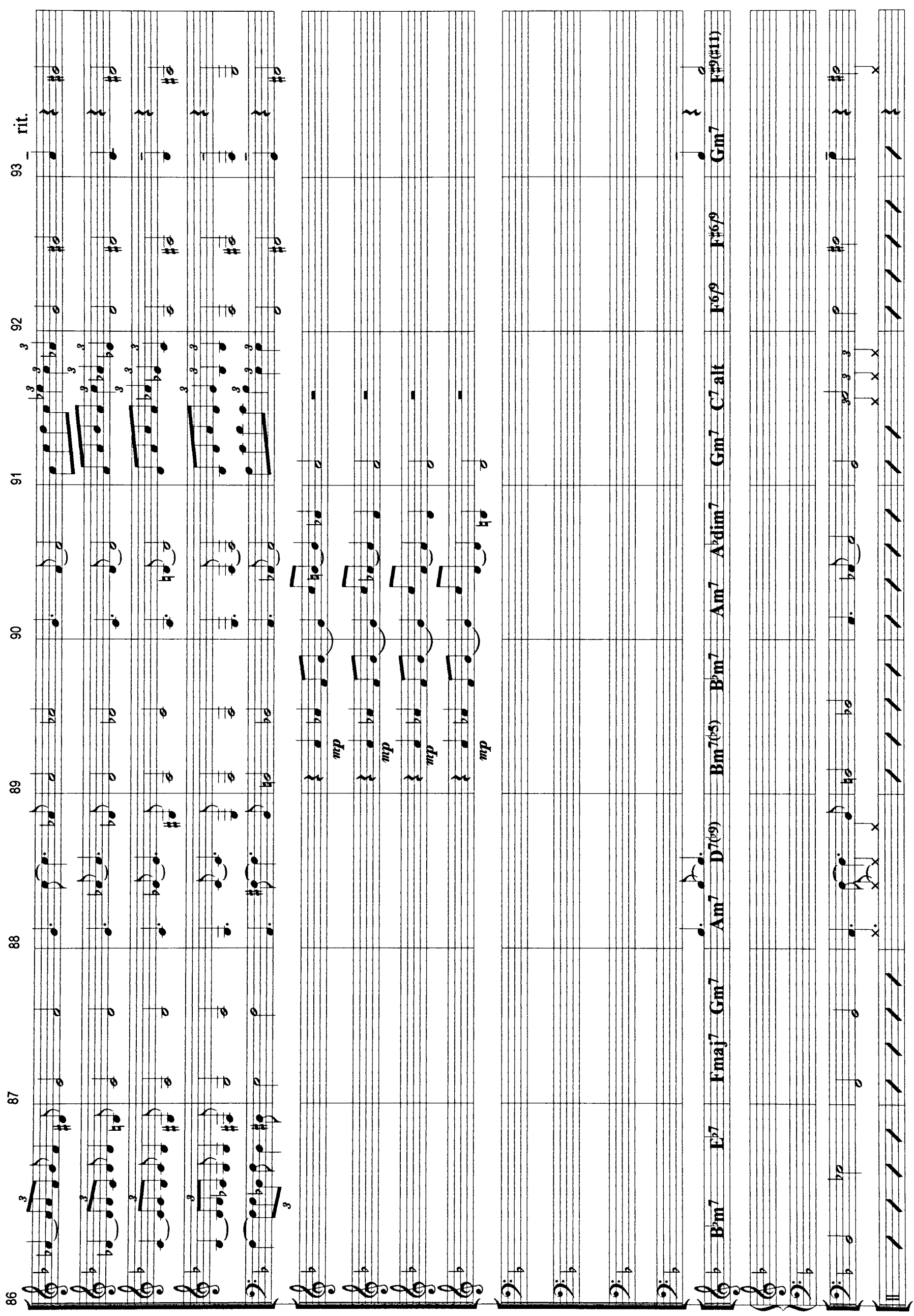




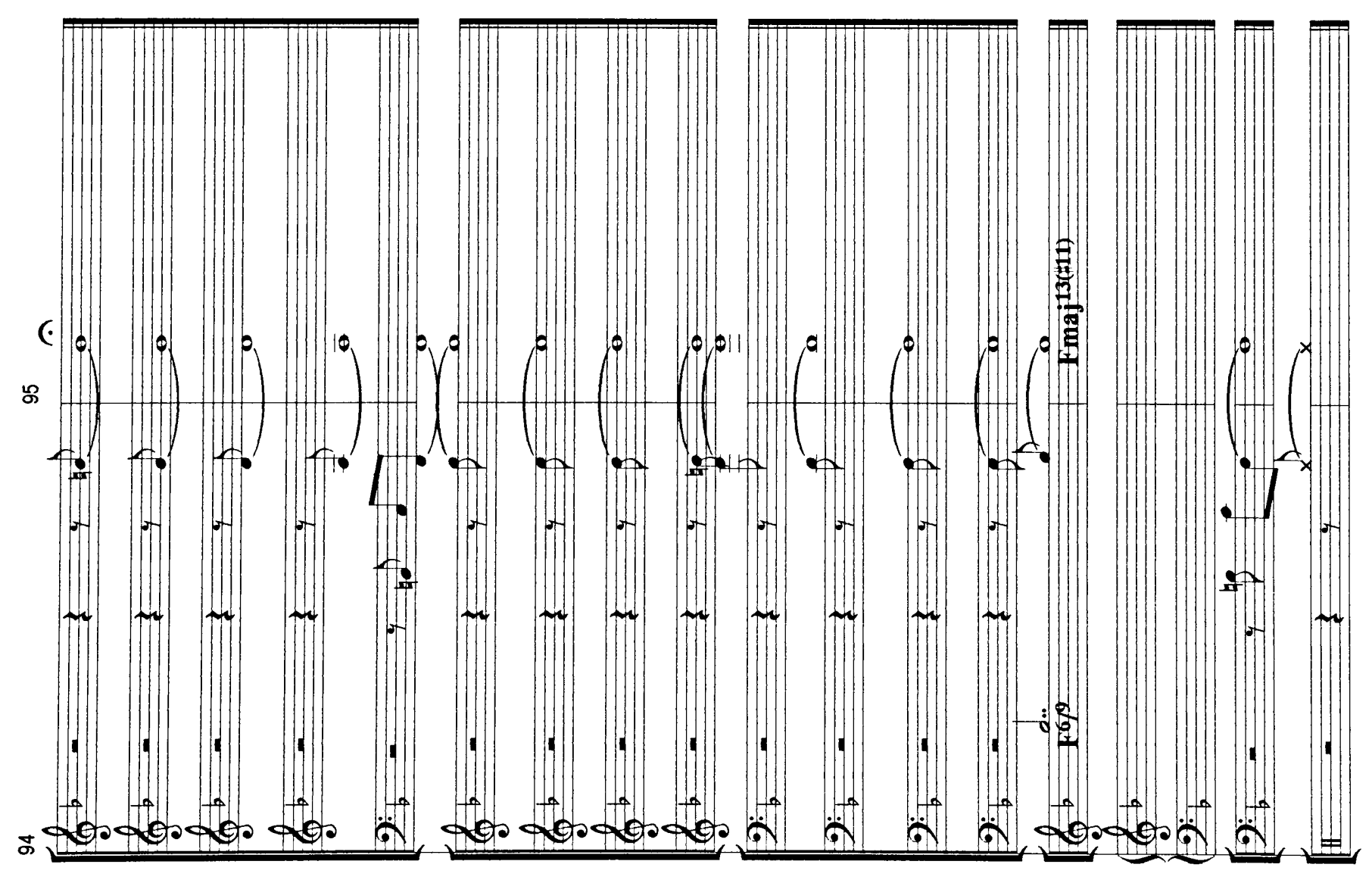




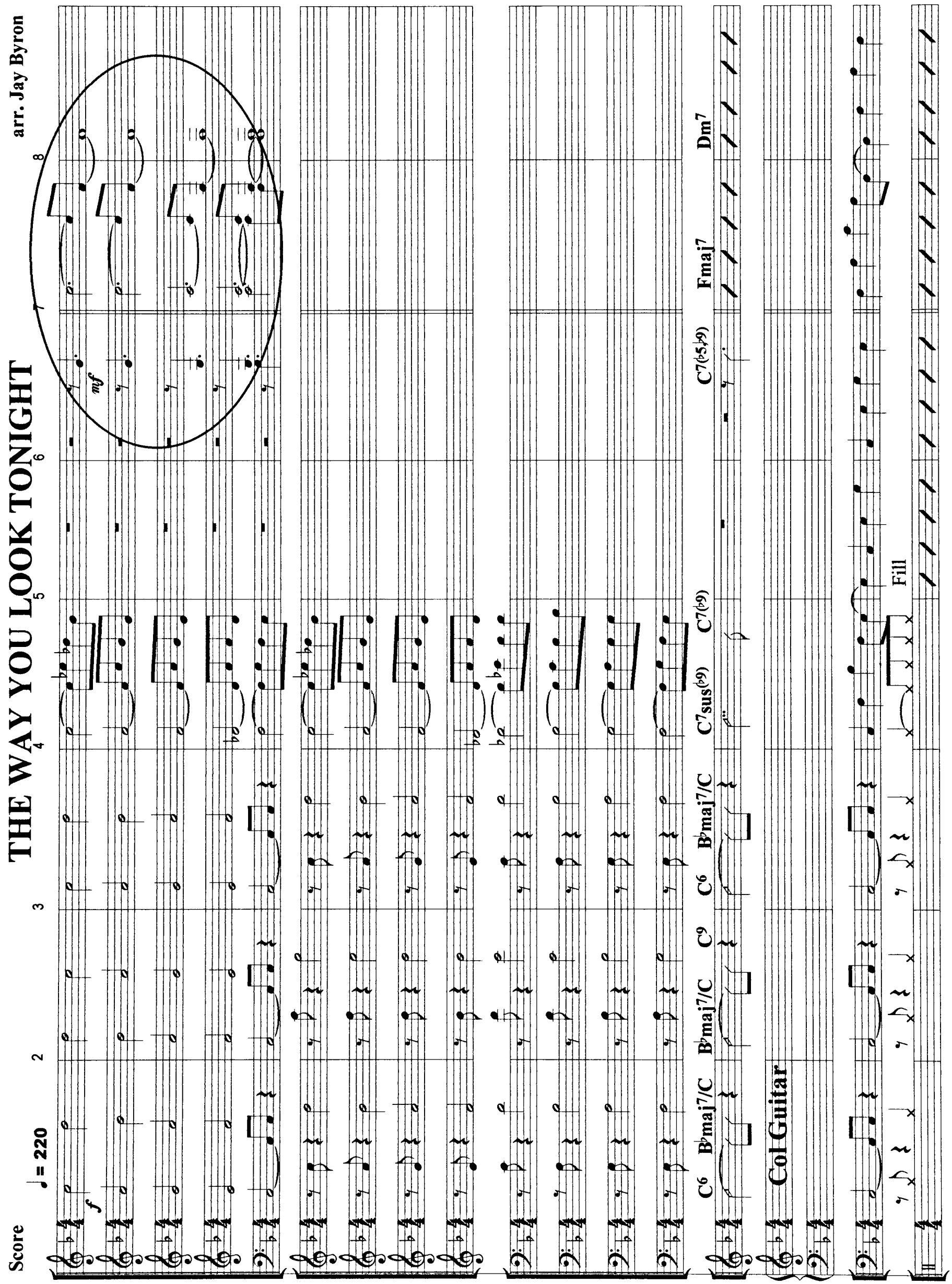




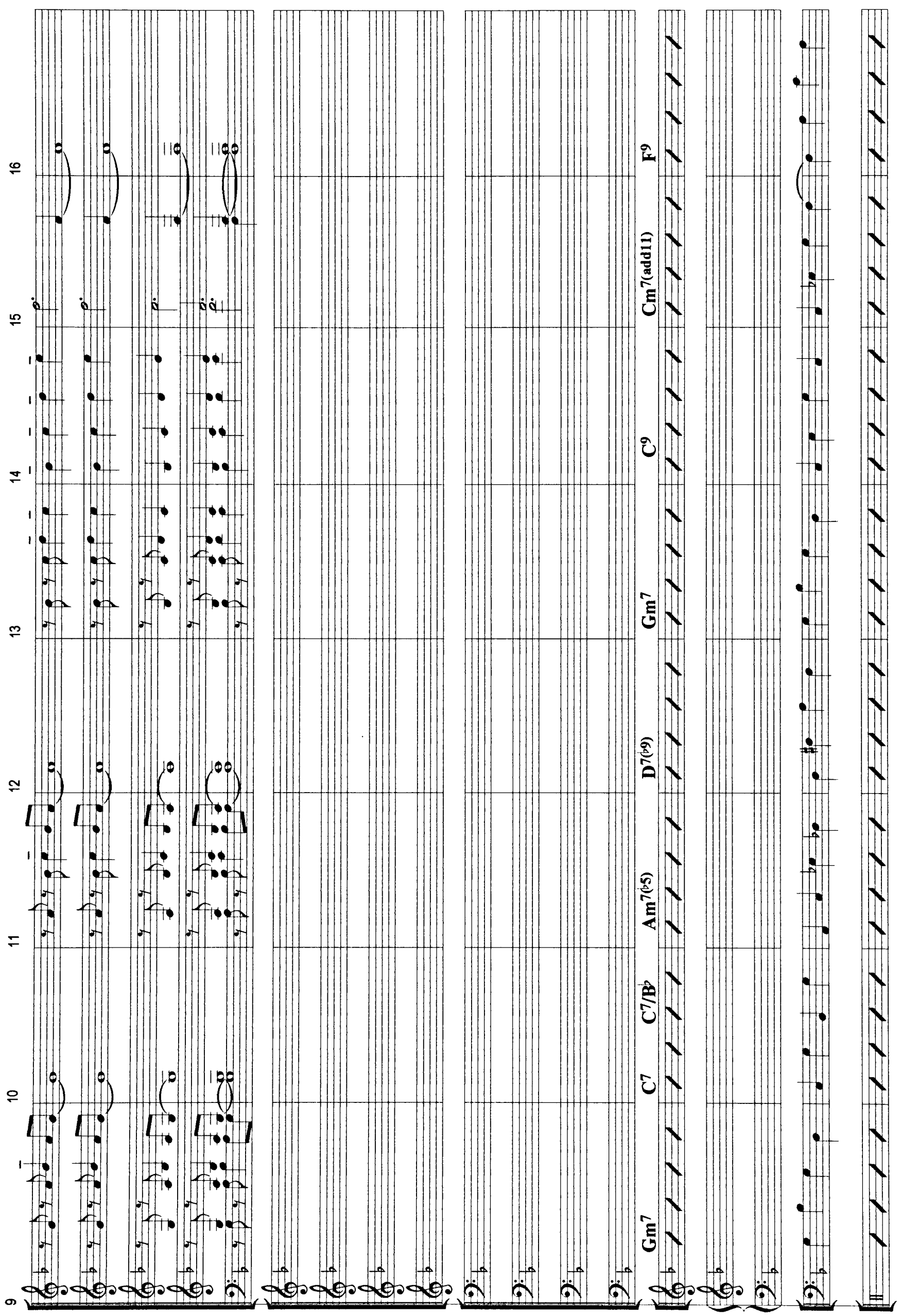




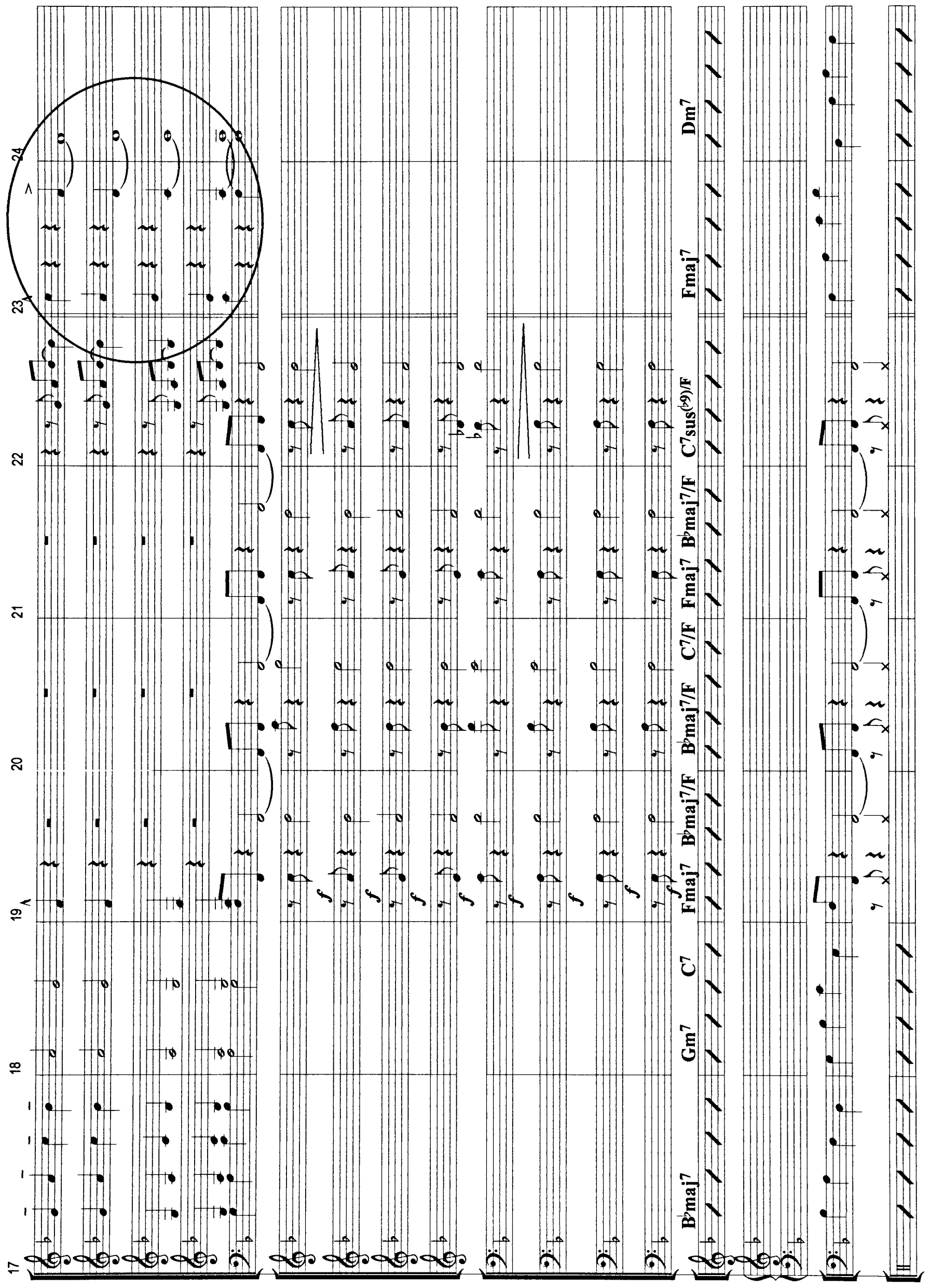



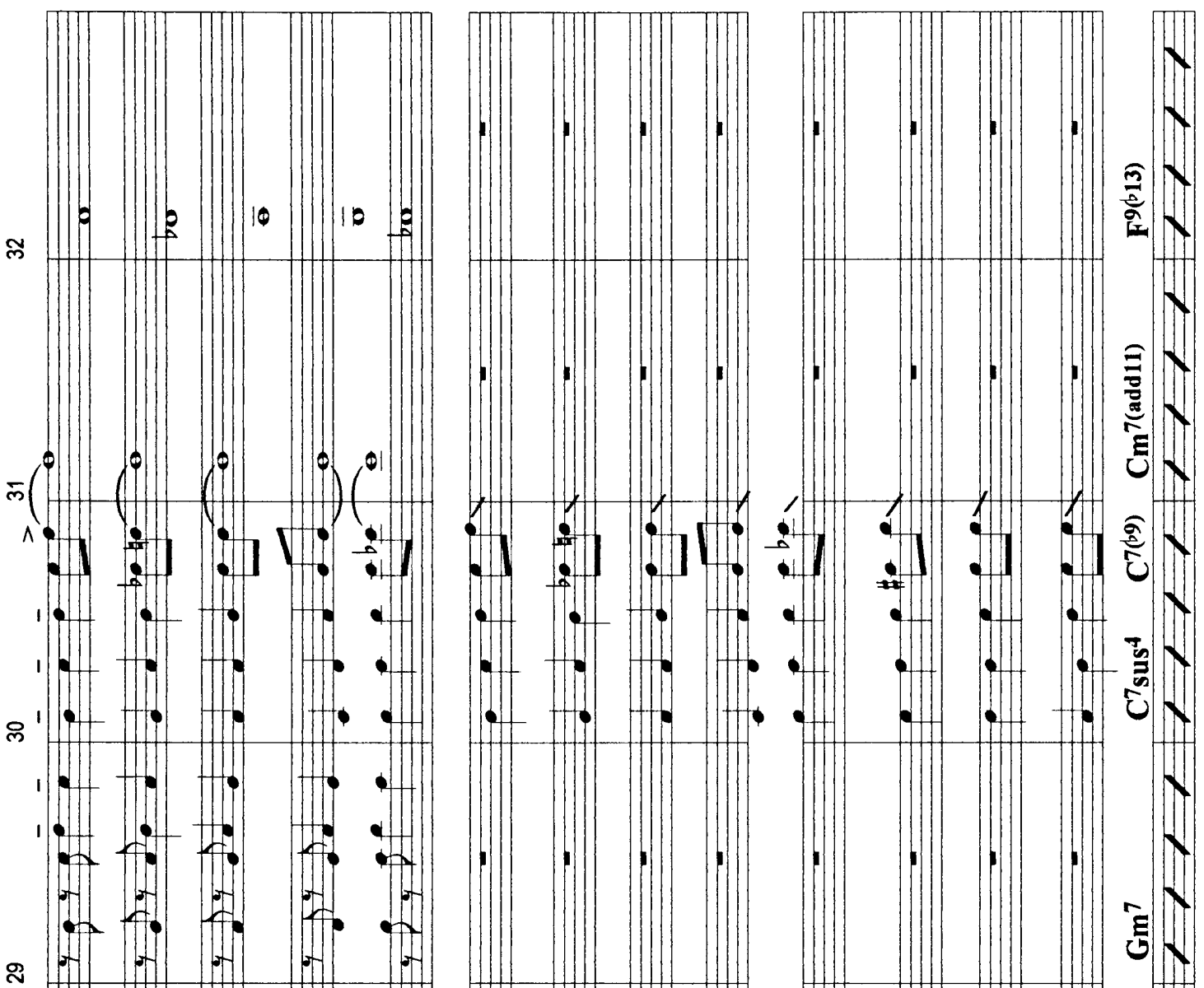

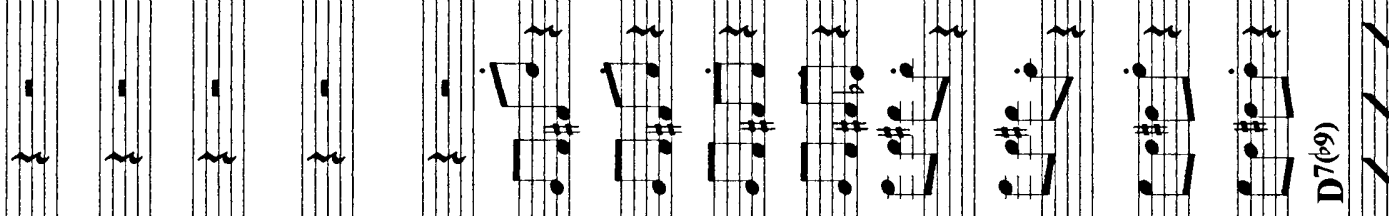

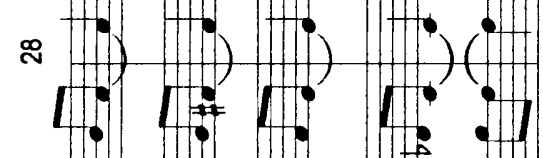

\section{$\sim \sim-\infty$}

-

$+4+4$

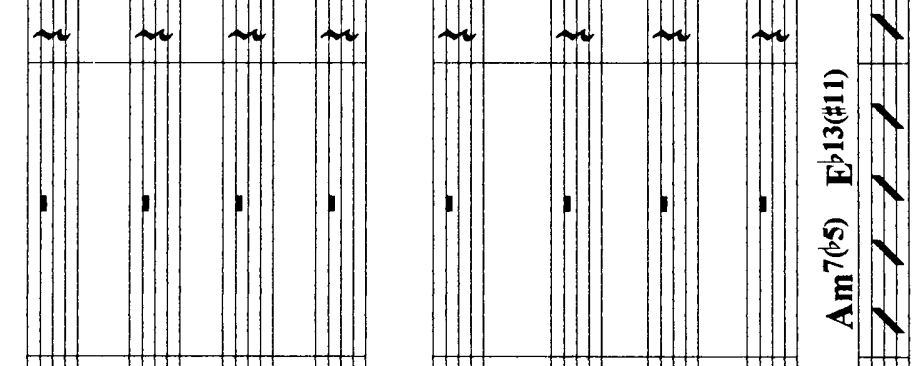

$\approx$ 我

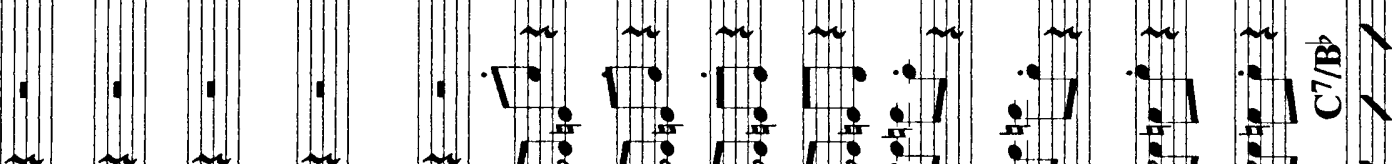

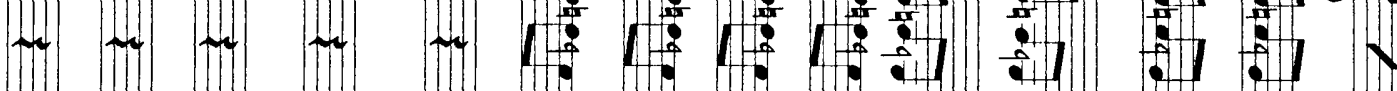

210.

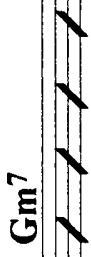

1.

H.

5

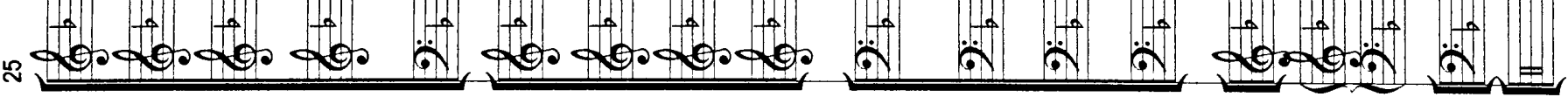




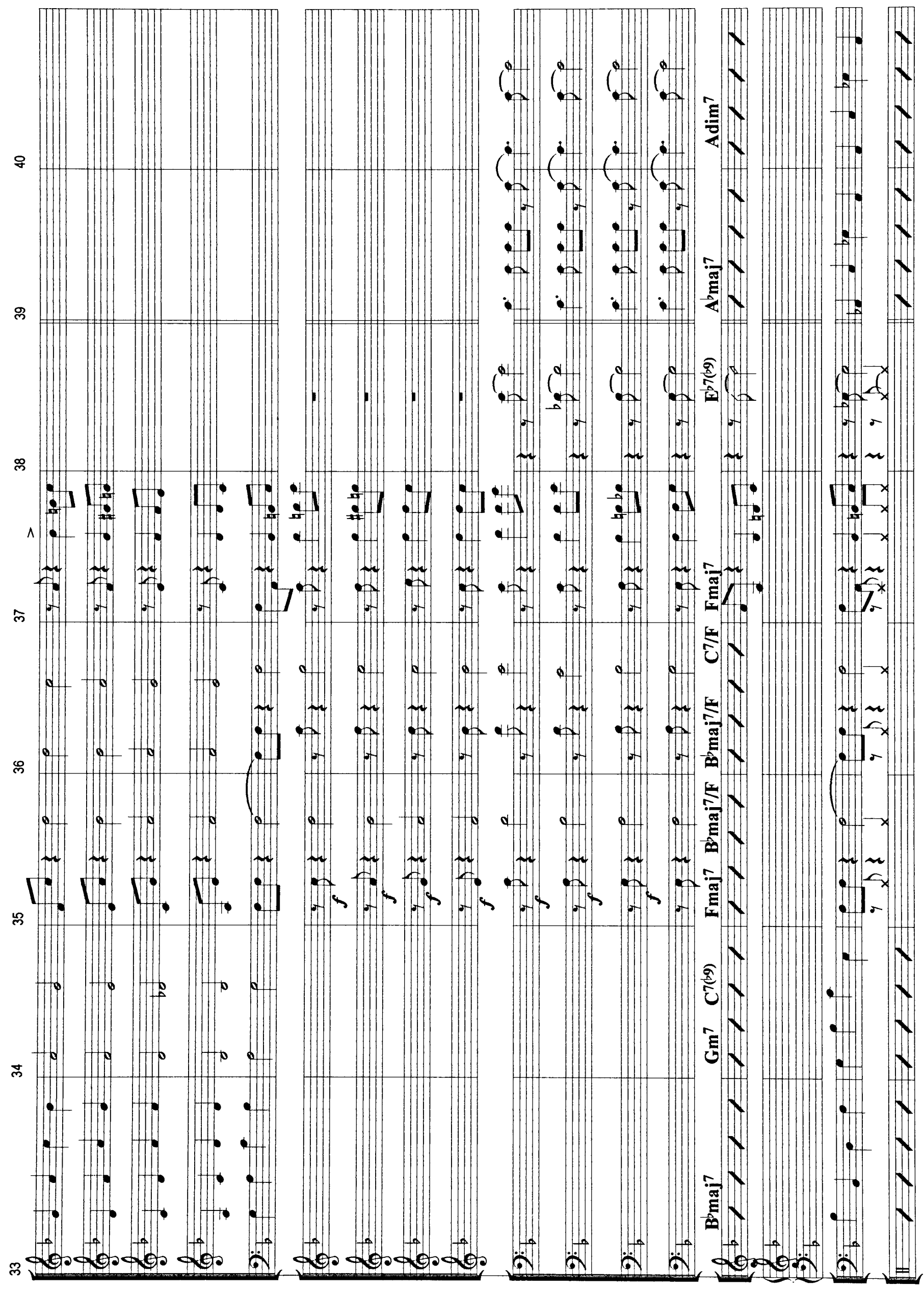




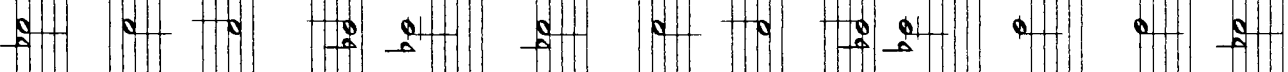

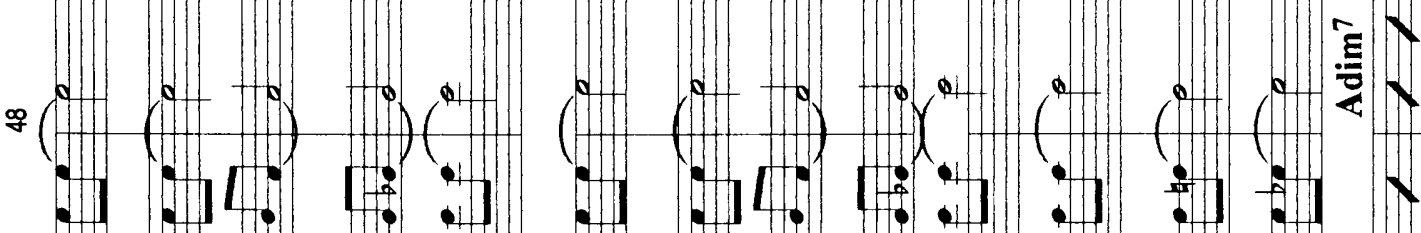

$3 \times$

3.190

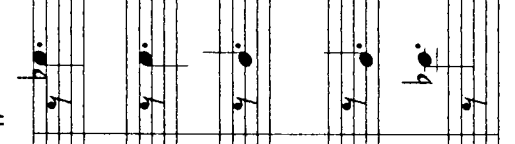

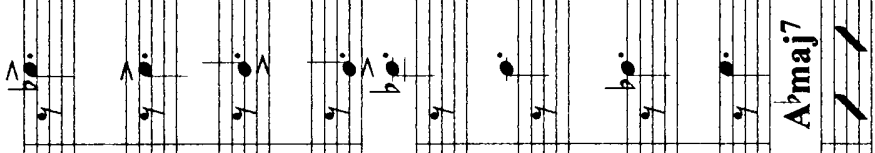

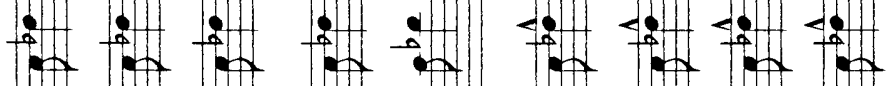

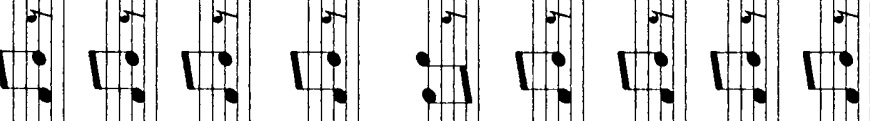

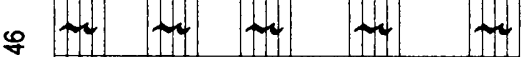

$+4+\infty$ d a d d

$\rightarrow W$

- N

8

a. 2 o \&

N

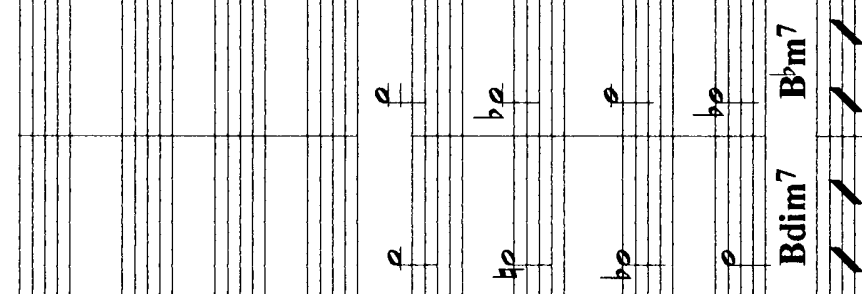

F
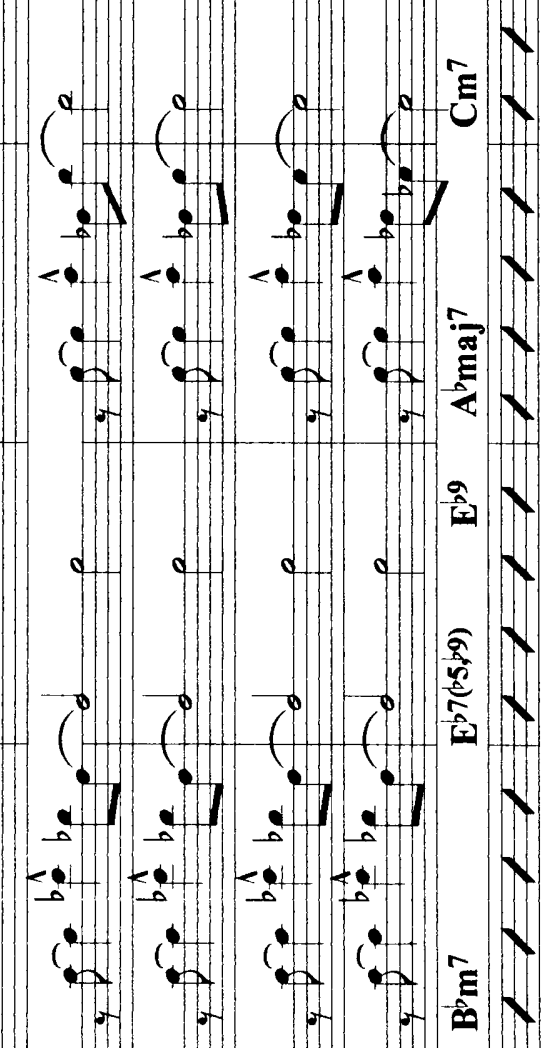

-

- $X$

₹

$\because N$

- $X$

- $N$

. $N$

- V

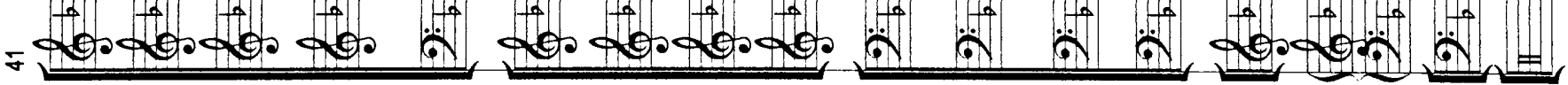




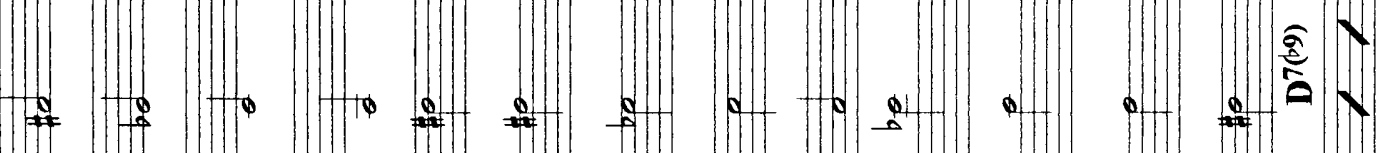

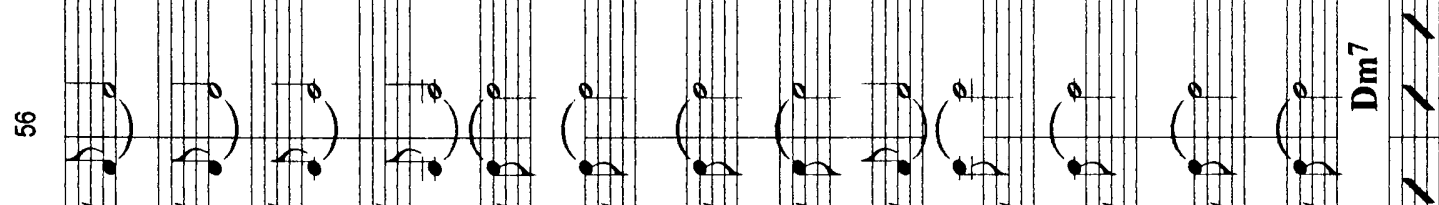

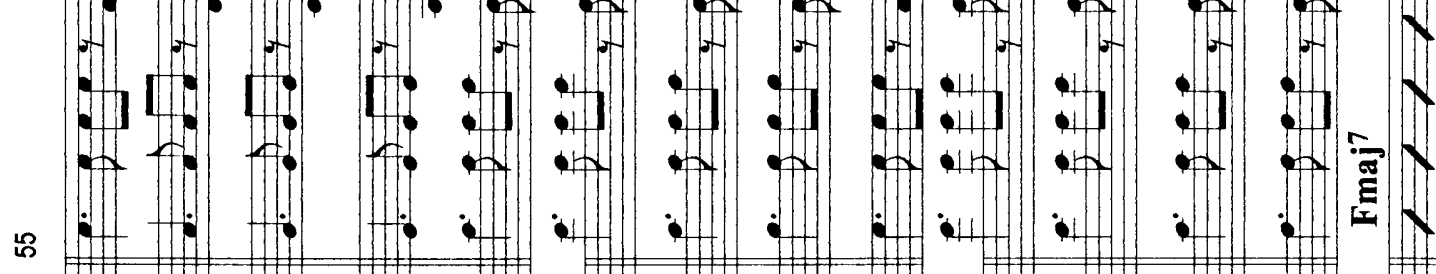

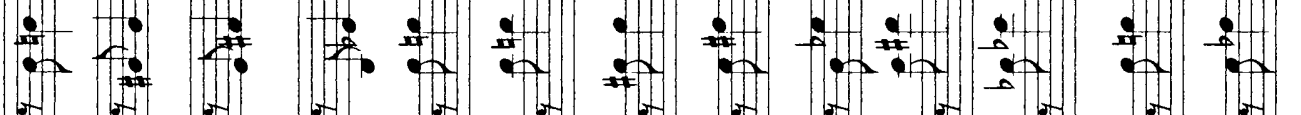

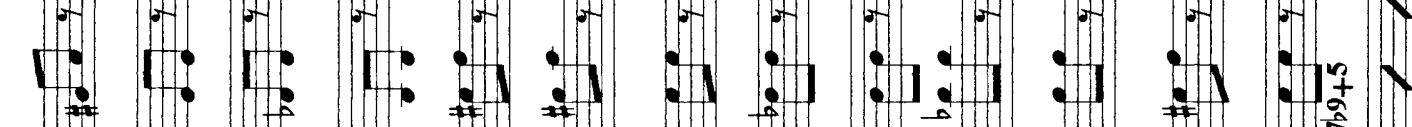
$\Xi \sim \sim \sim \sim \sim \sim \sim \sim \sim \sim \sim \mathcal{\sim}$ 

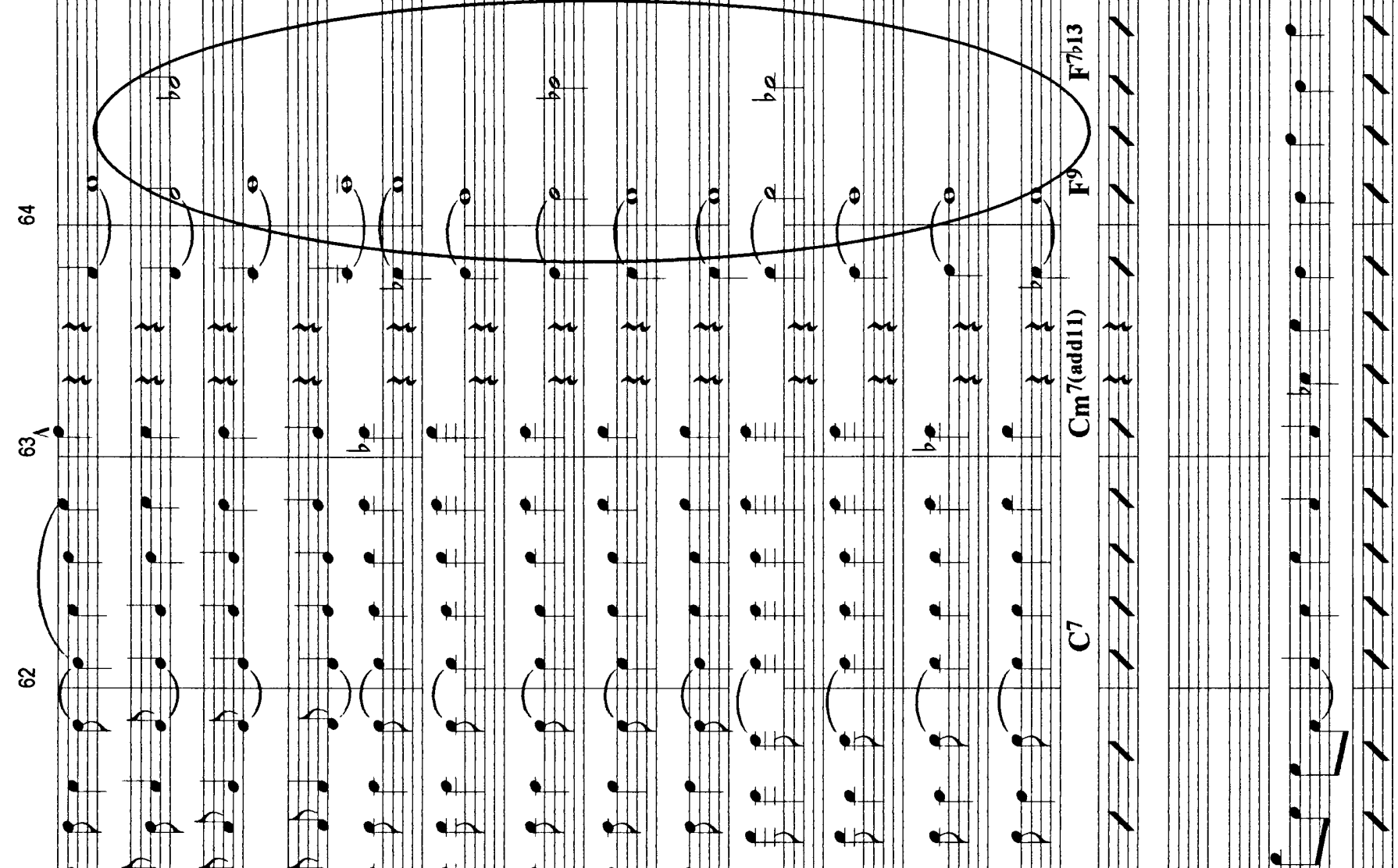

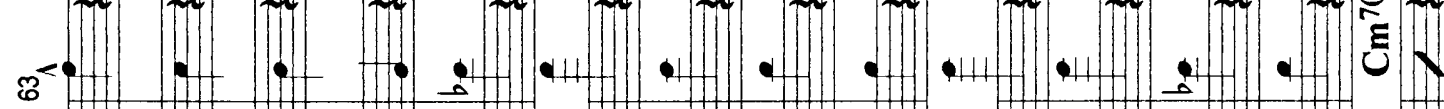

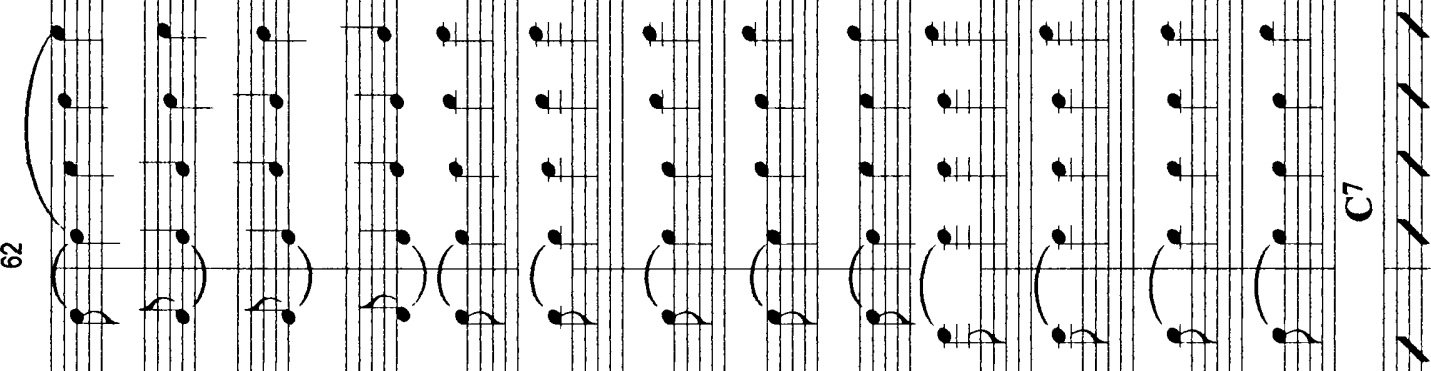

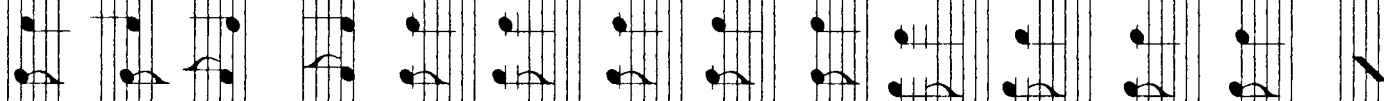

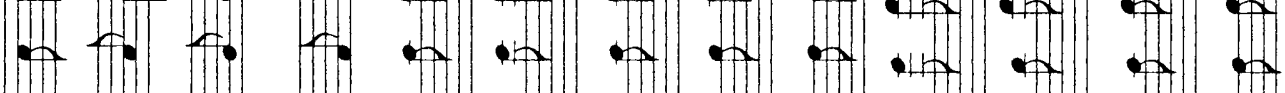

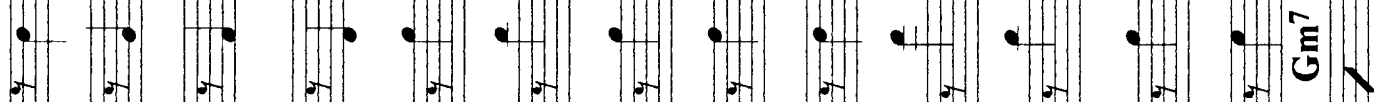

$\bar{\omega}$

*

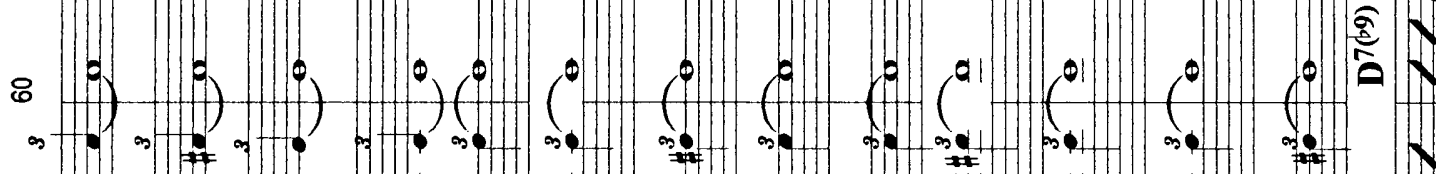

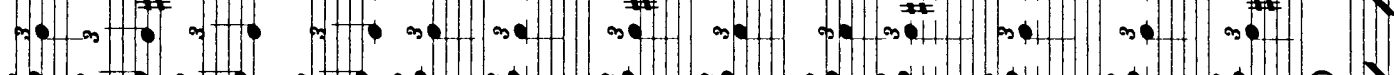

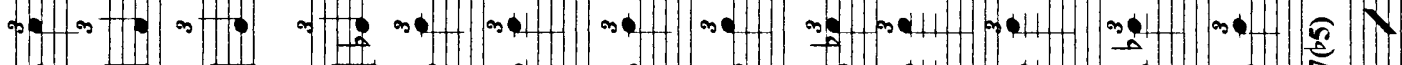

I.

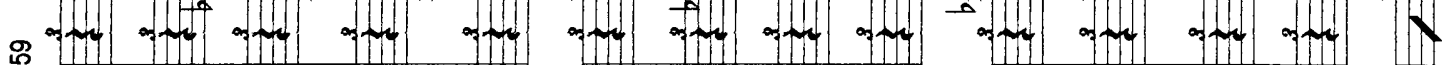
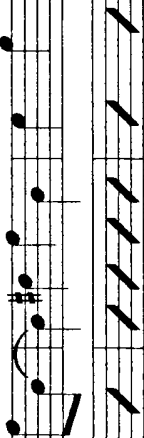

- 1

+N

$x$

$1 \ln _{0}$

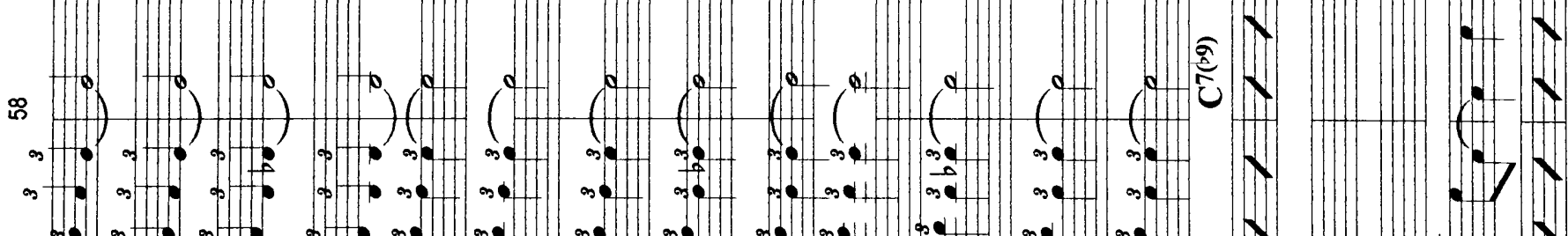

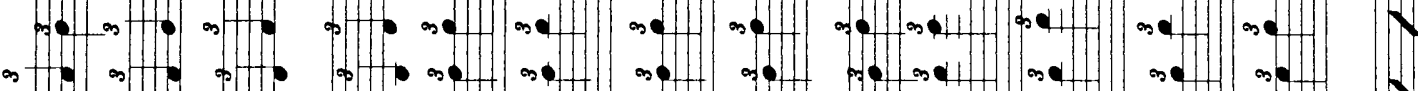

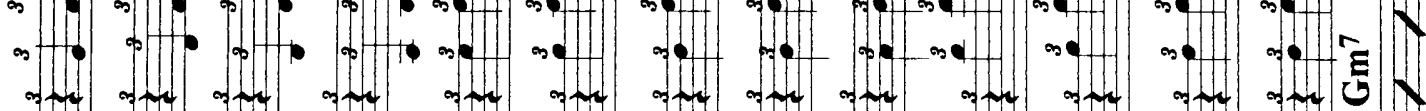

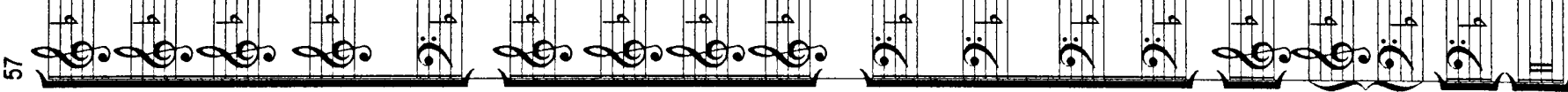




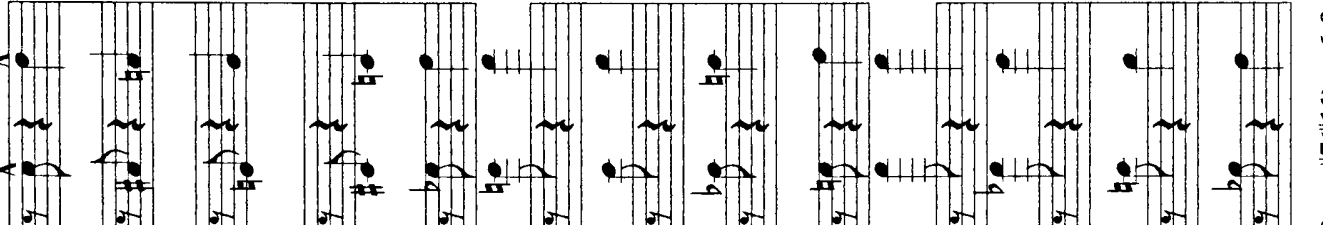

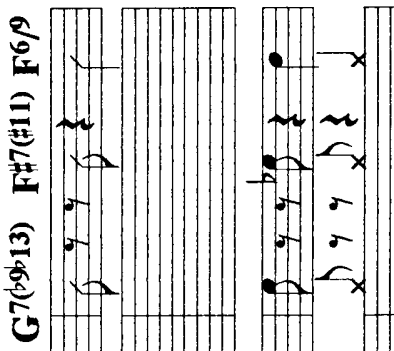

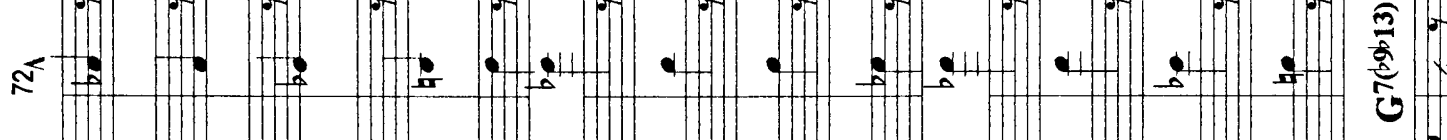




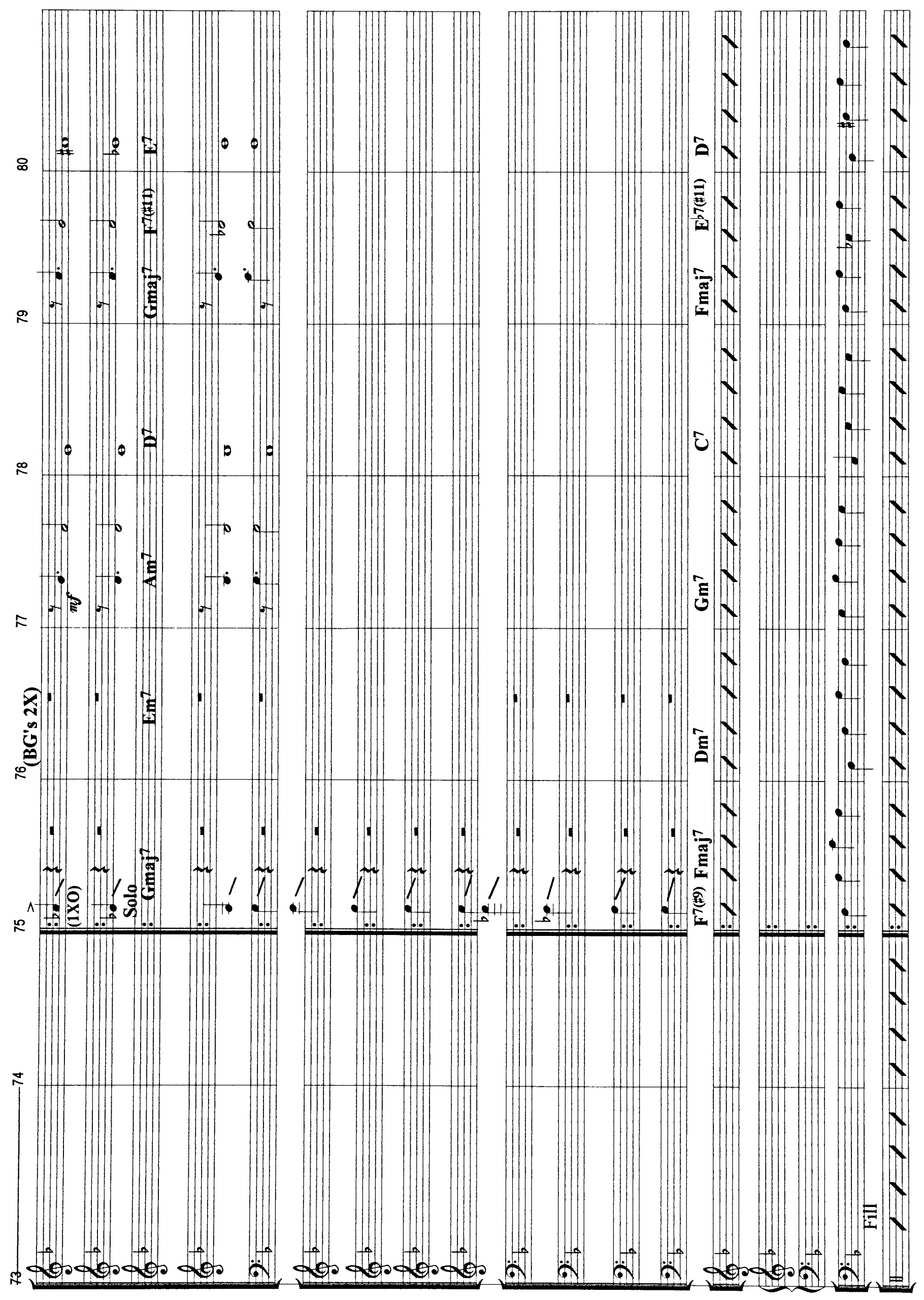




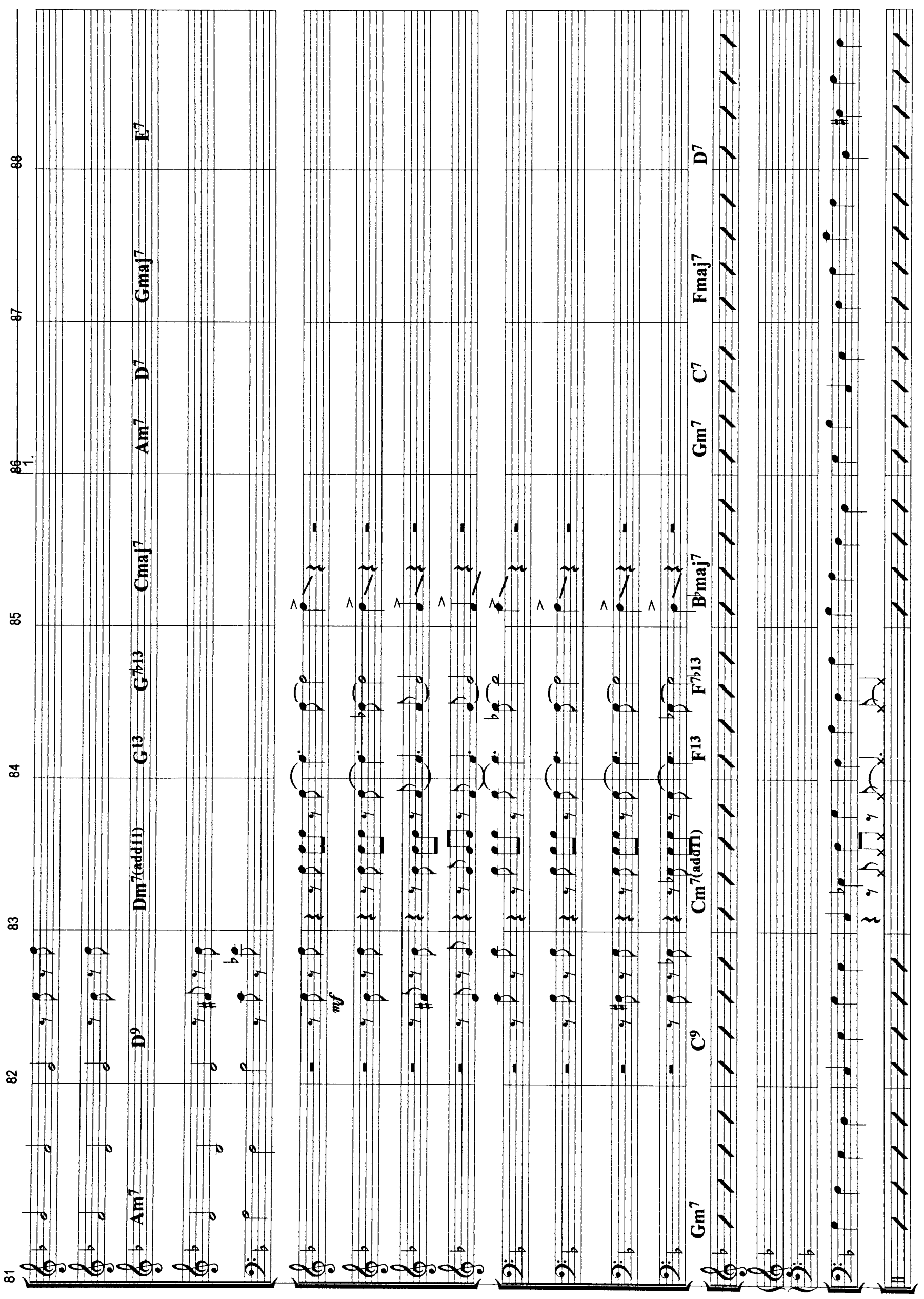




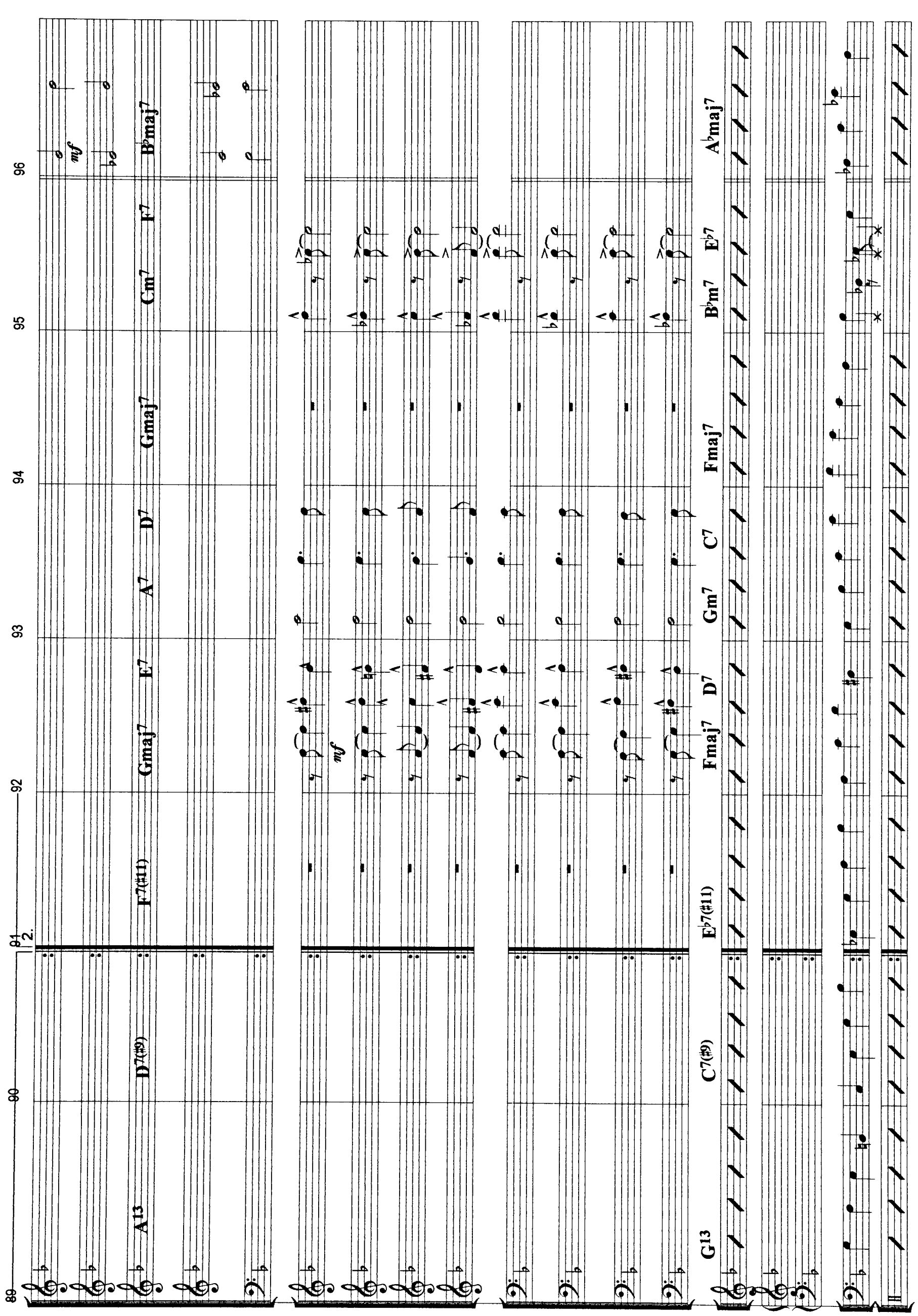



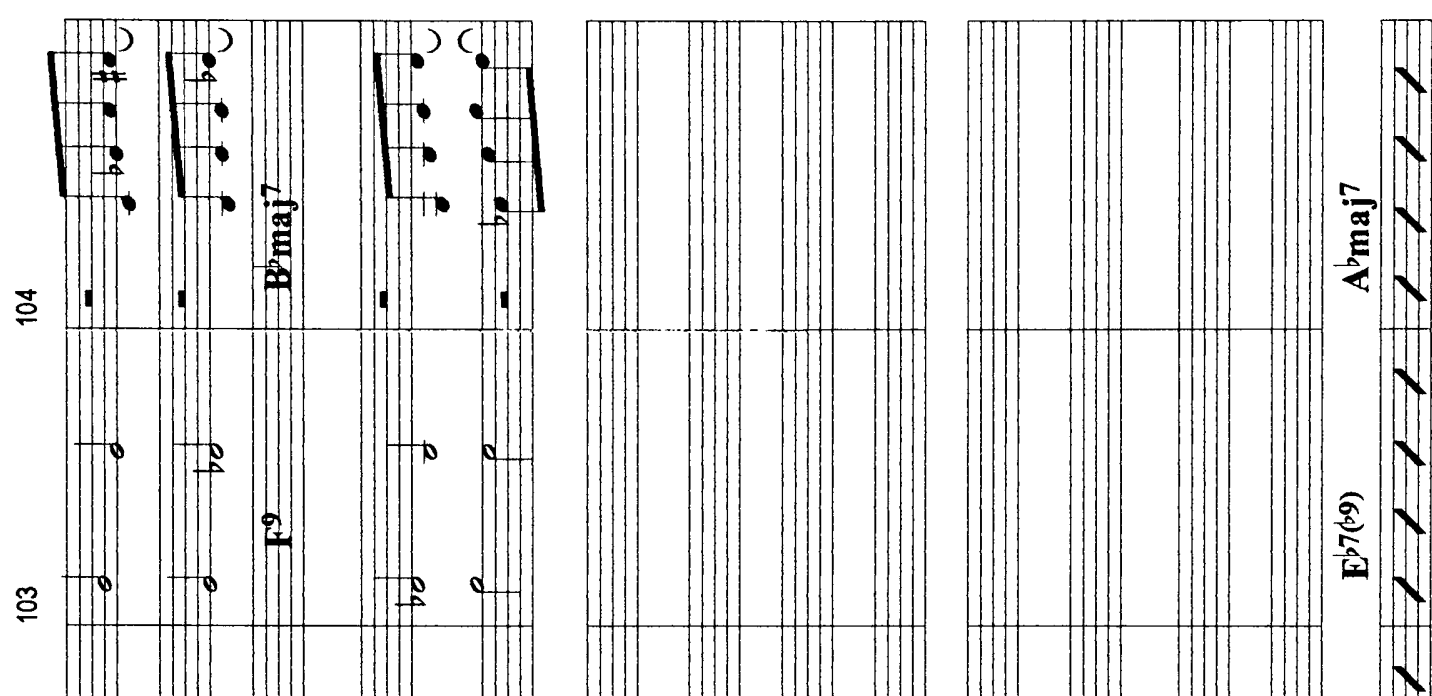

$+\infty$
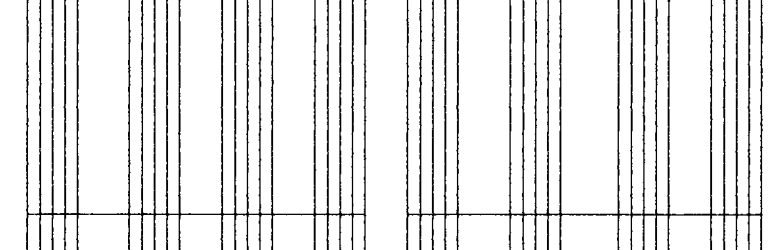

ธ $.1+0$ I
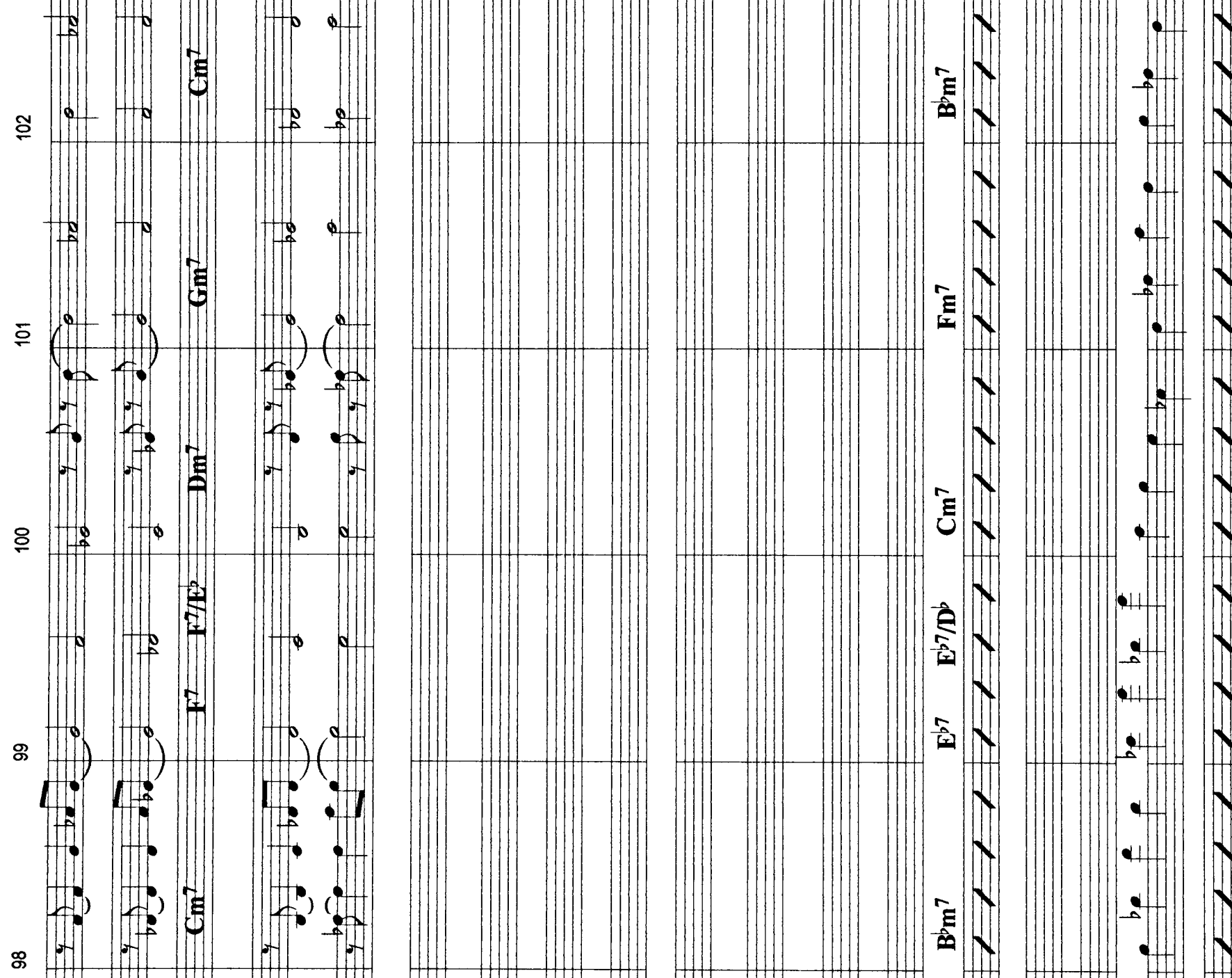


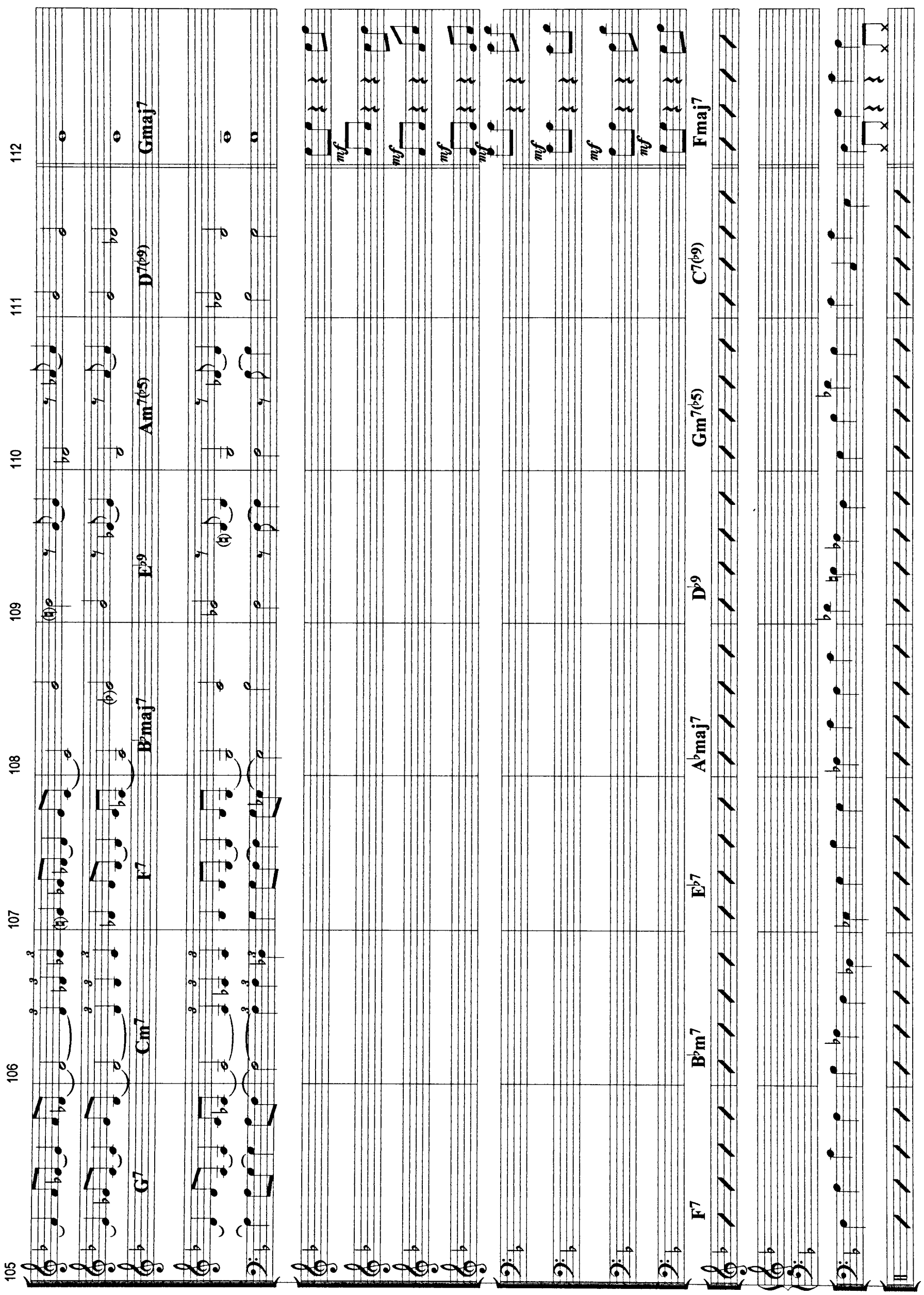




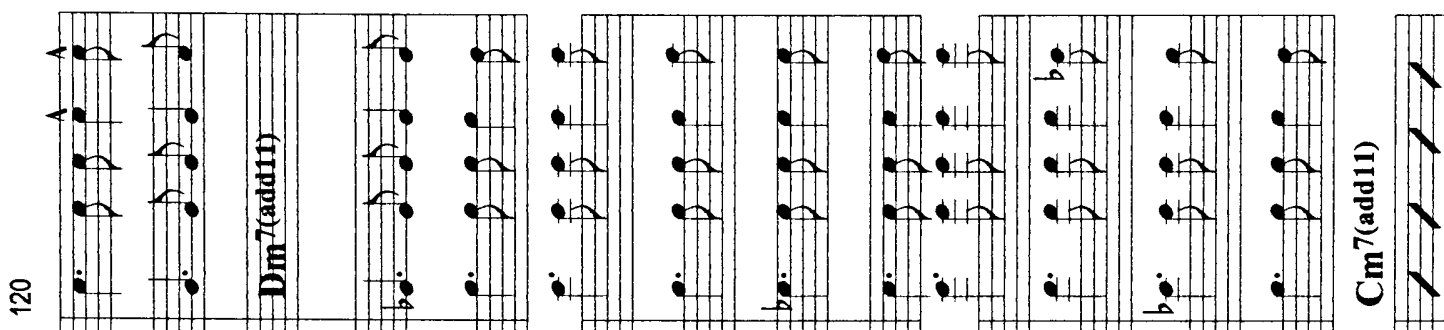

$\div \div \div \div \div \div$

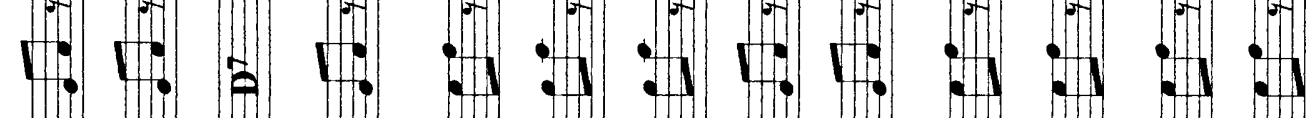

$=$ m. m:

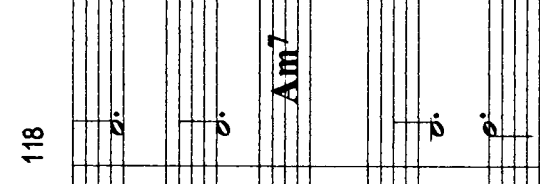

$\stackrel{\infty}{*} \|_{1}$

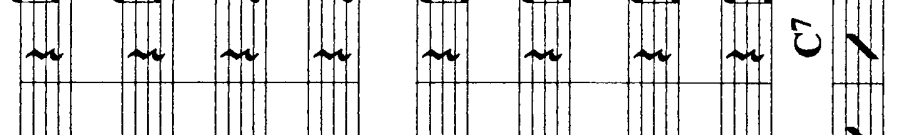

$\sqrt{4}$
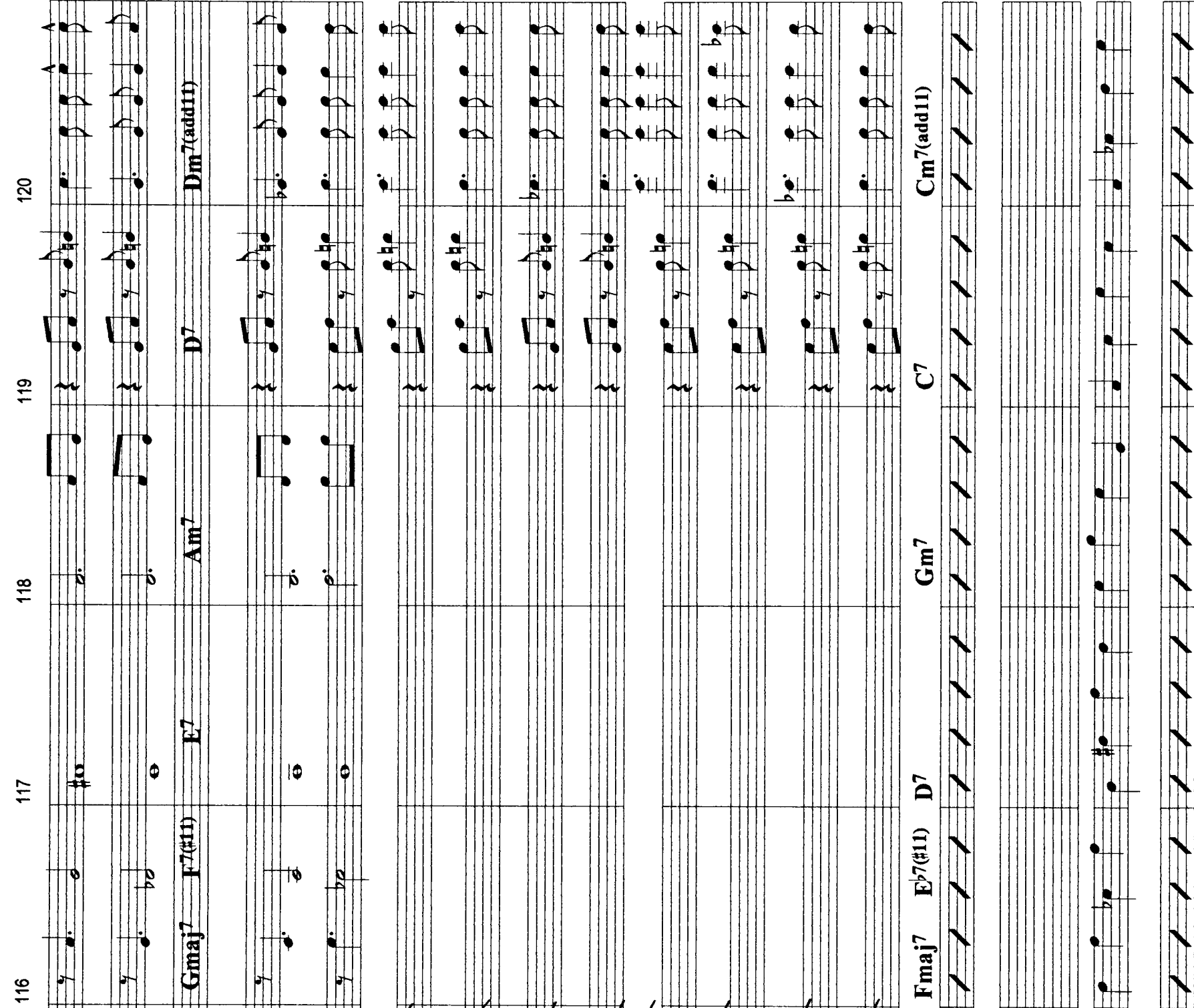

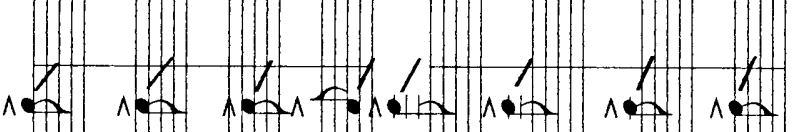
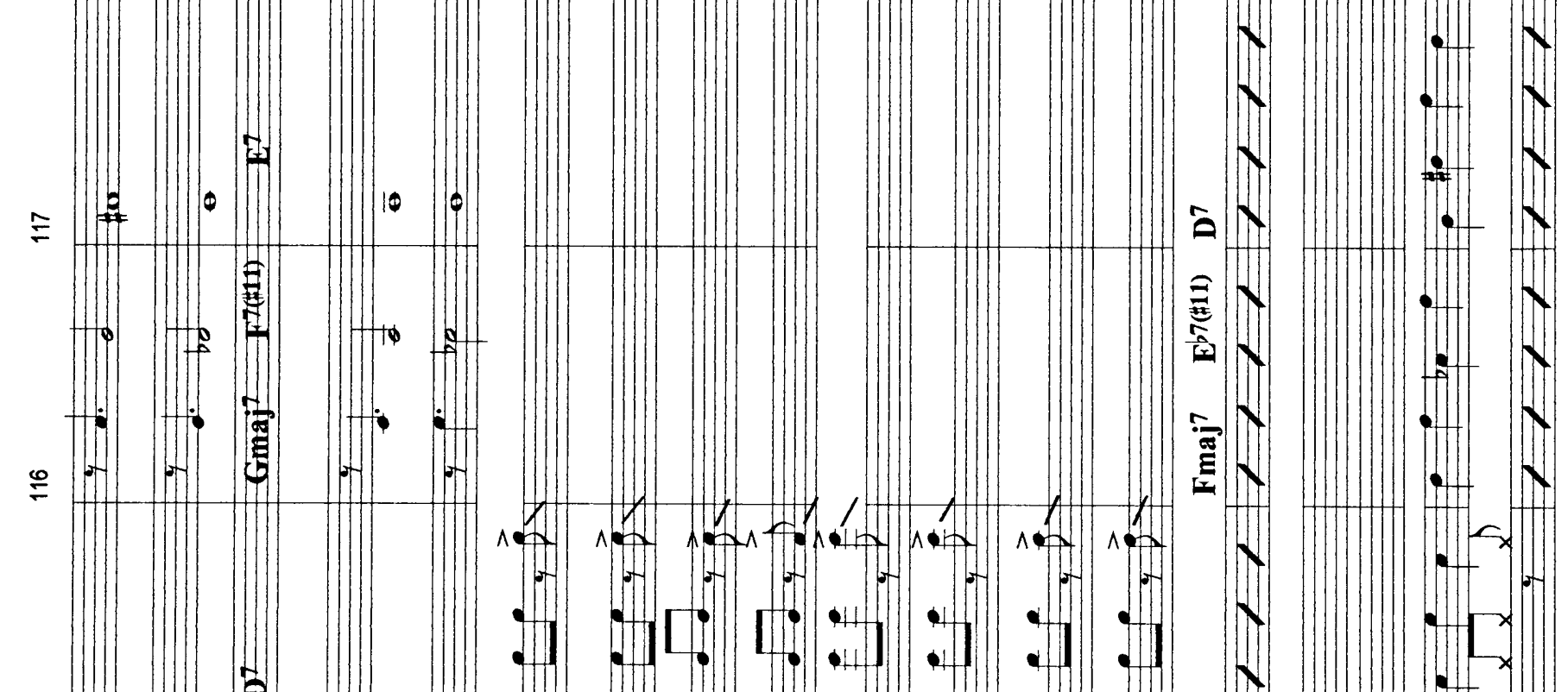

$\because$

$=$

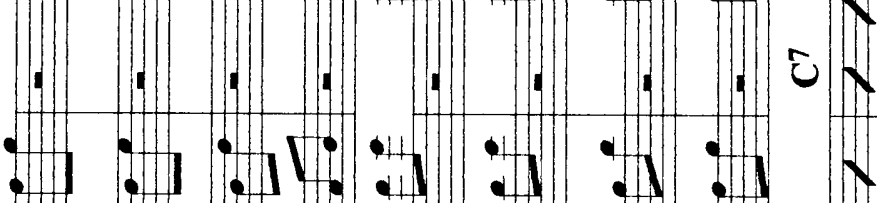

184

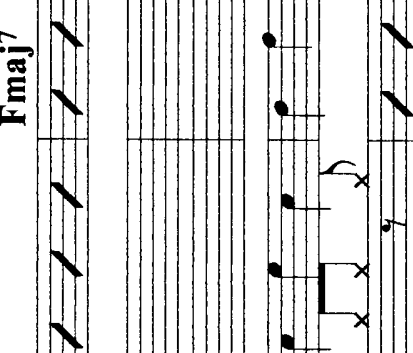

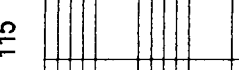

: $15:$ : $15:$ :

$\rightarrow$

$\cdot \sqrt{2}$

$-\infty$

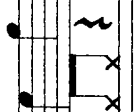

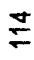

i

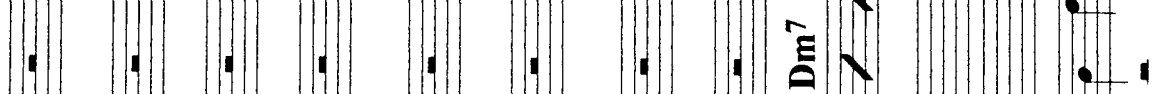

- $x$

$1+x$

.

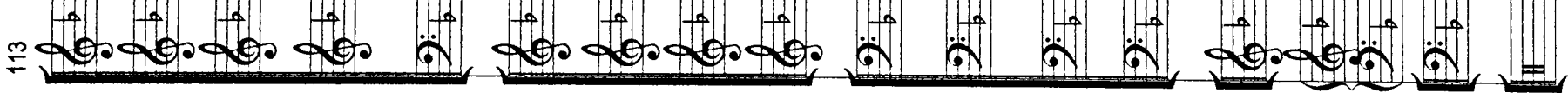




\section{$\div$ - $: 4$}

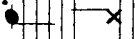

$\cong$

H.

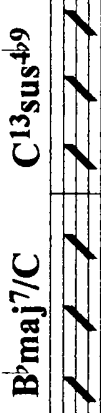

$\stackrel{2}{2}$

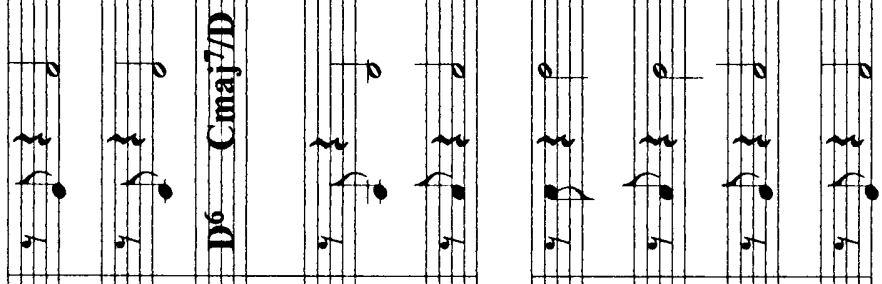

ن

Hin

T.

¿े

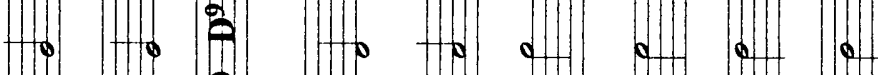

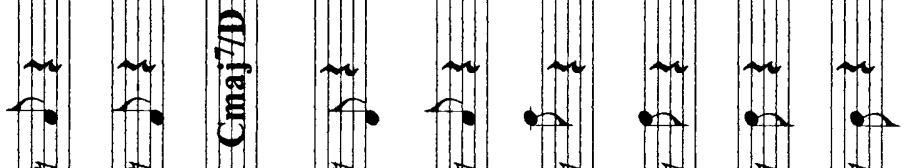

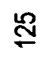

$\rightarrow$ a $\rightarrow{ }^{\circ}$

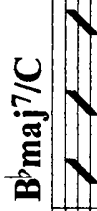

$+x$

H.

尝)

$\circlearrowright$

$\pi m$

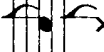

$\rightarrow 0$

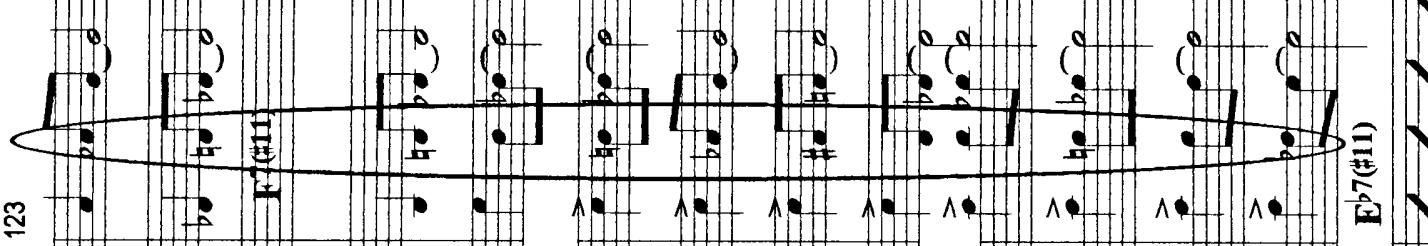

N.

- 1

- $N$

$2 \sqrt{2}$

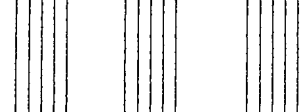

$\underset{(H)}{(1)}$

$<+<<-1<<<+1$

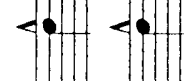

-
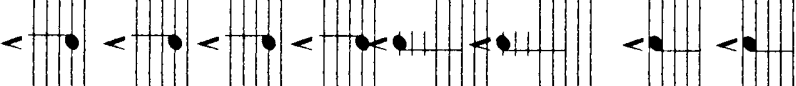

N

(ข) $:-$

ฐ

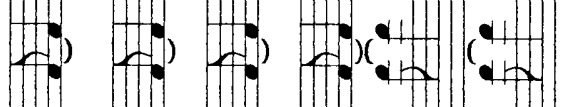

$\rightarrow$ an $\rightarrow$

$+7$

11

. 110

- 1.11

$\sqrt{1}$

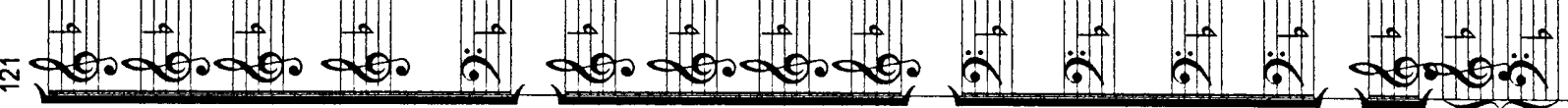




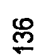

๓

"ே

誉

్

흐

옴
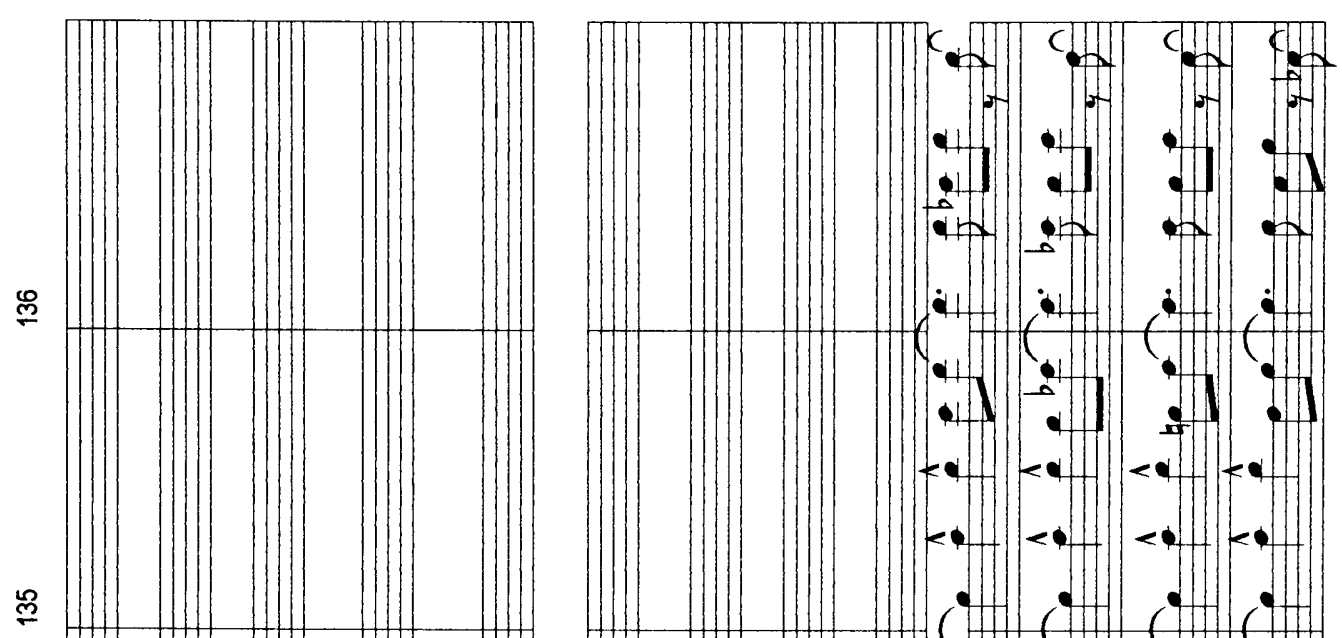

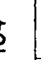
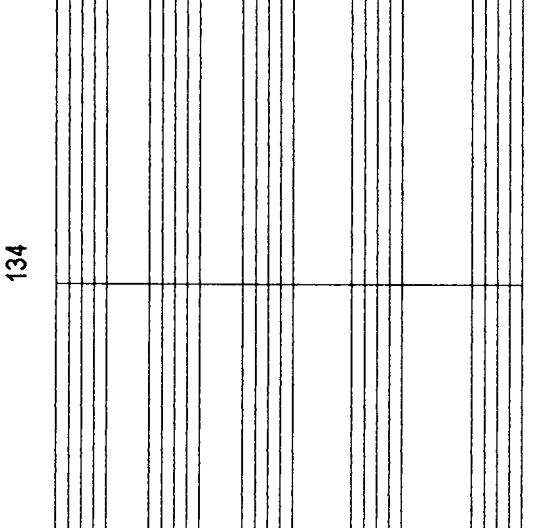

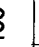

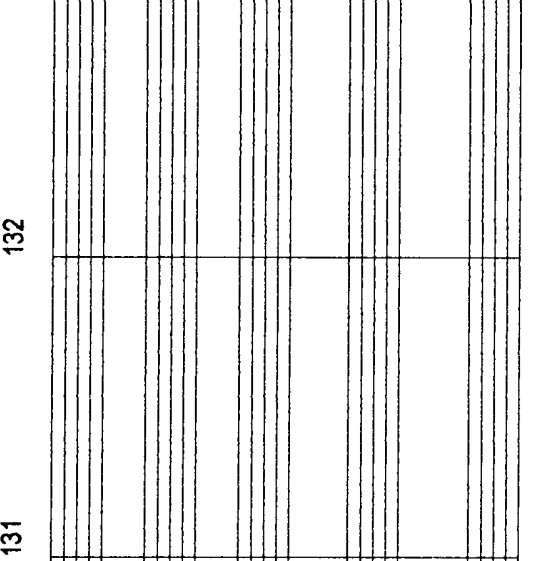

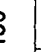
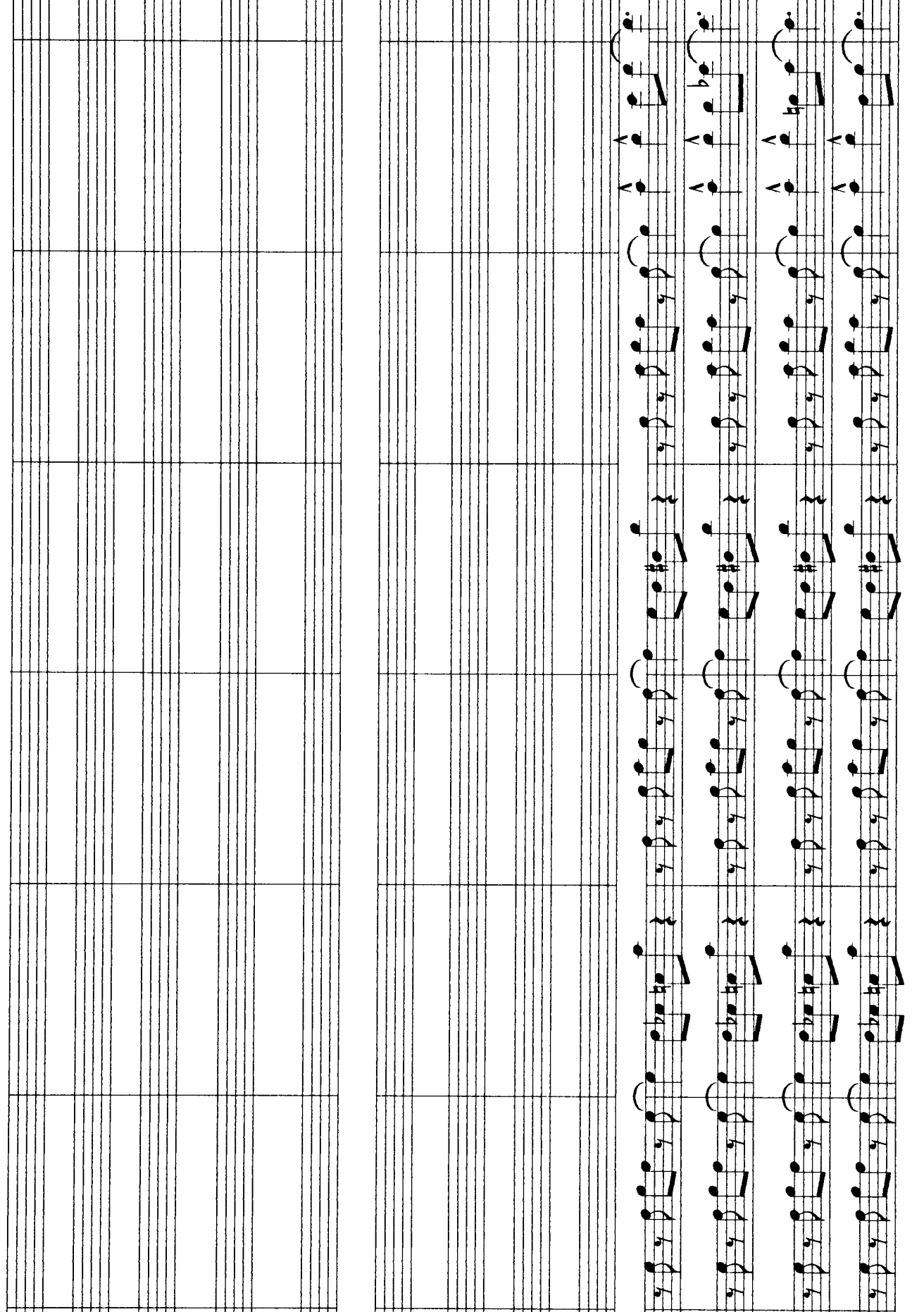
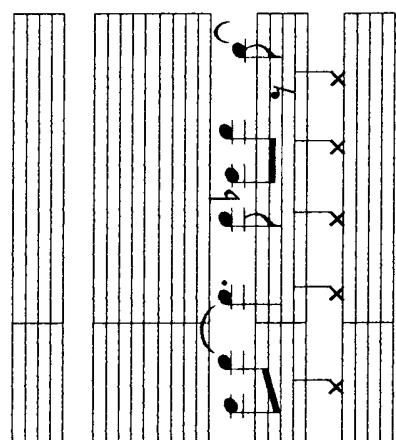

1)

- $x$
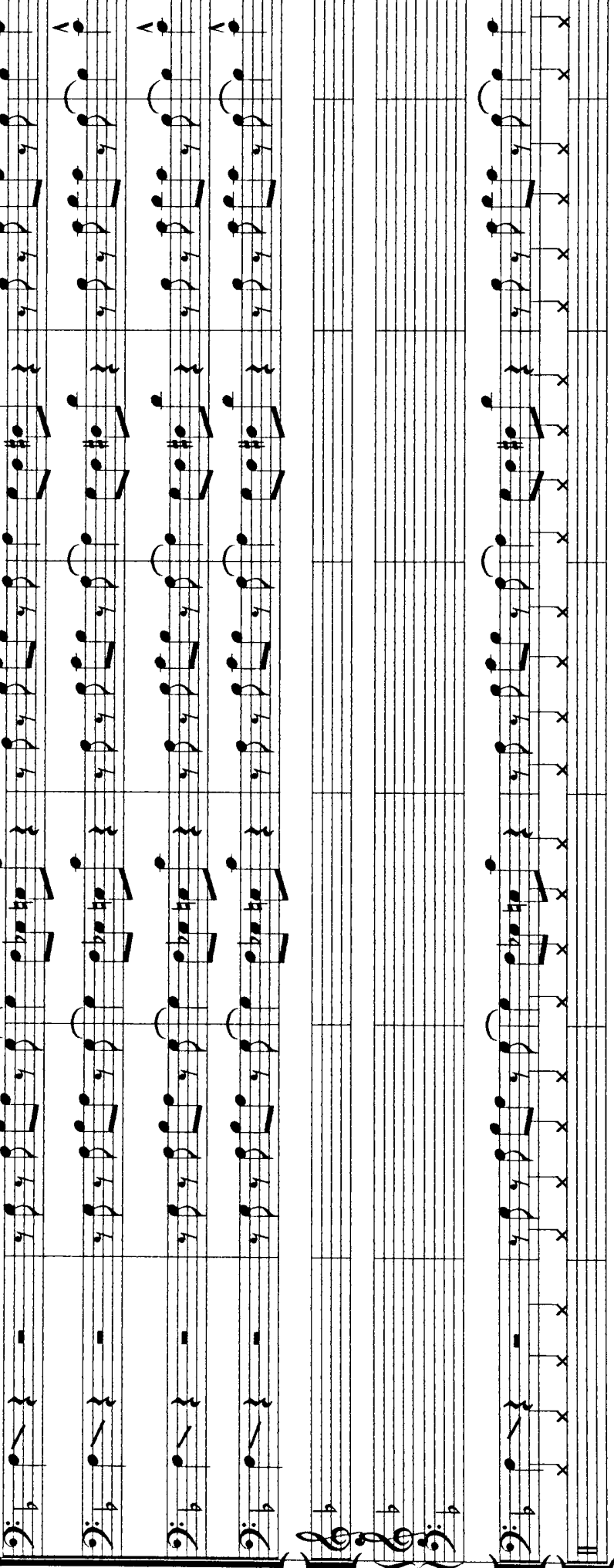


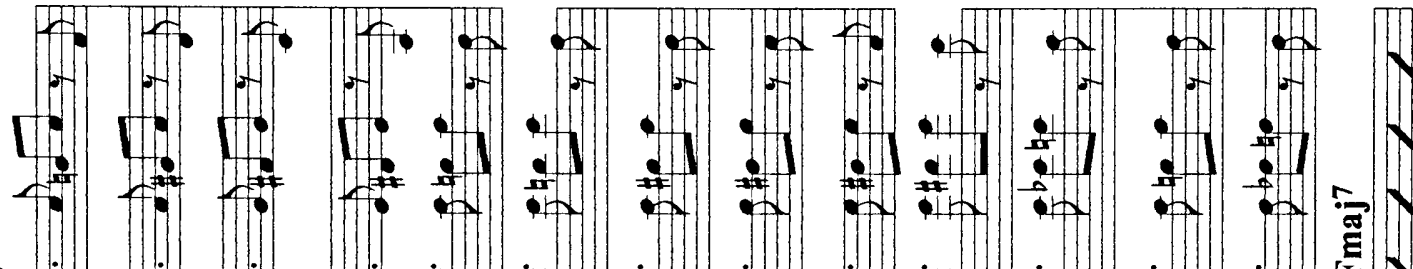

$\sqrt{1} \sqrt{ }$

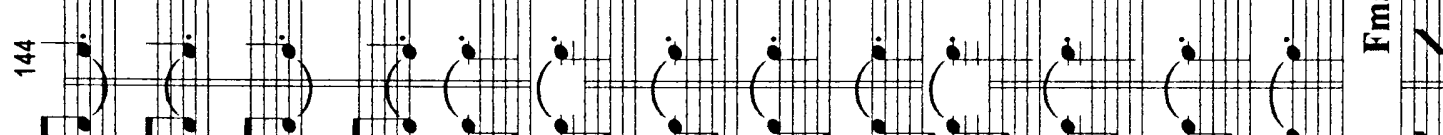

10 10 :

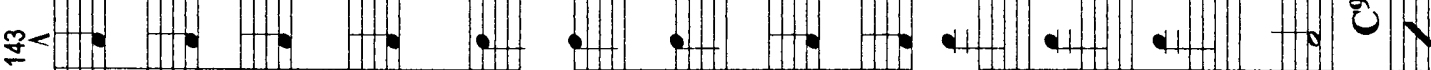

$+1+1$

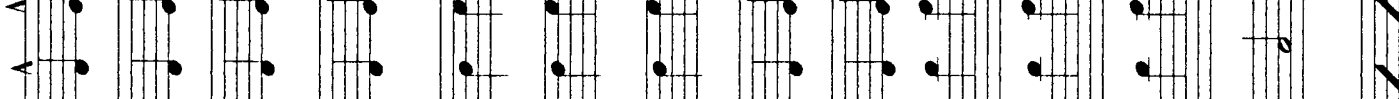

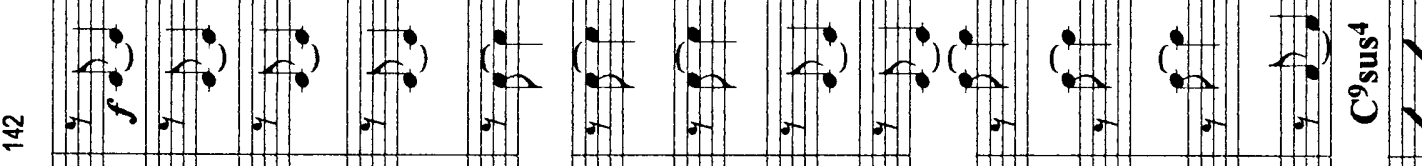

an $\cdots$ and

10

||

110

$\Xi$

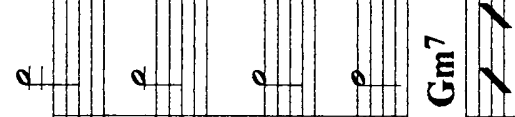

1.)

P

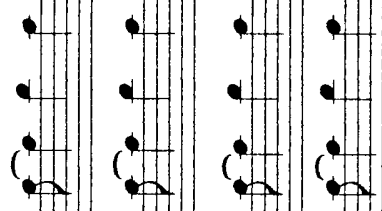

- $1 N$

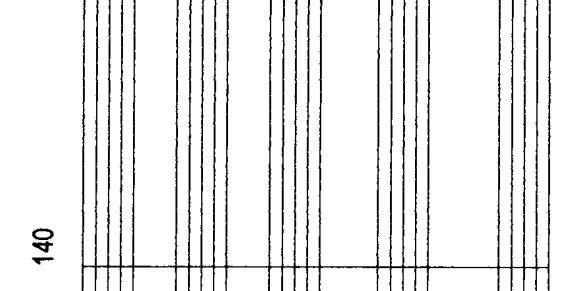

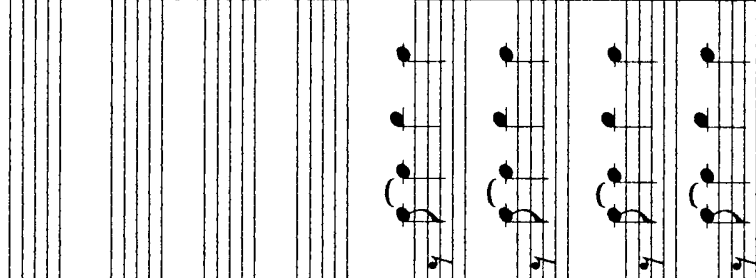

$$
\text { \# }
$$
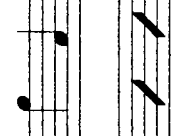

- $N$

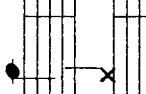

- 4

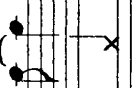

-

$\rightarrow x$

(a) (o)

g

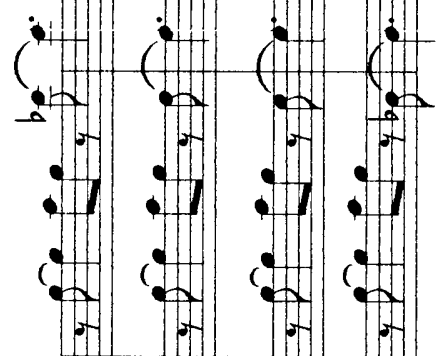

$(-\infty)+\infty$

- $x$

(1) $x$

$\infty$

$\rightarrow x$



1 II

$x$

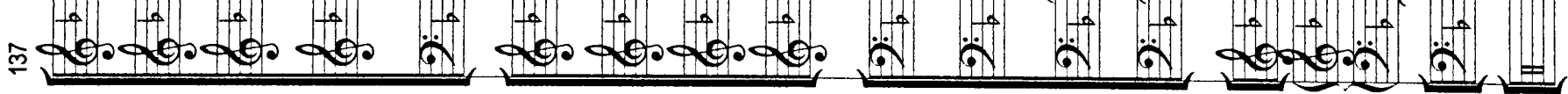


Sका

at ot ot at

- 1. 1.

(2)

- H. . . . . . . . . . . . .

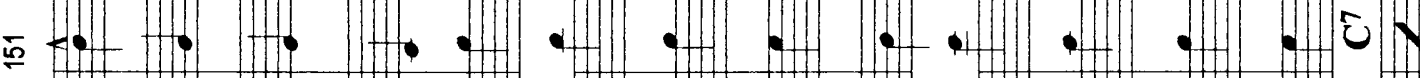

a

+. +. . . . . . . . . . . . . . . . . .

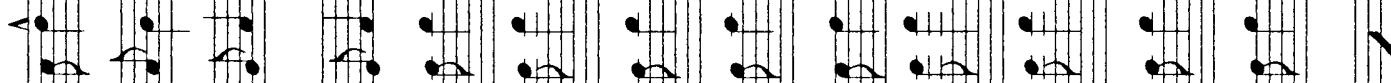

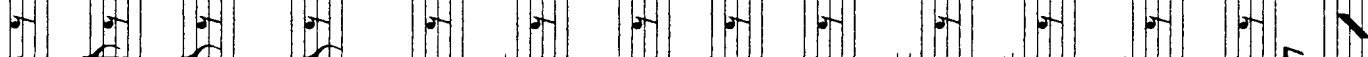

W

$4 \rightarrow-4 \rightarrow-4 \rightarrow+4$

4. L.

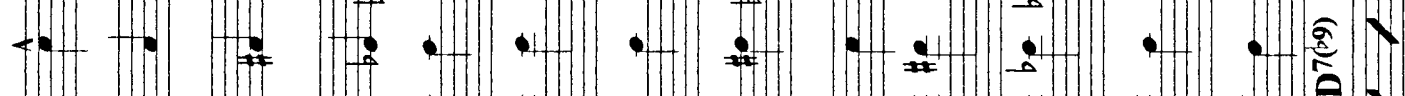

g. 40 -

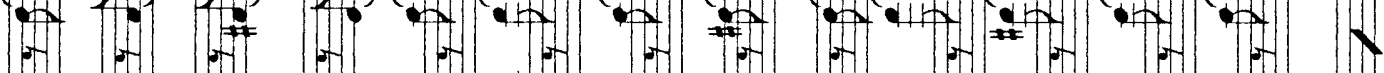

1. 50 15:

H f:

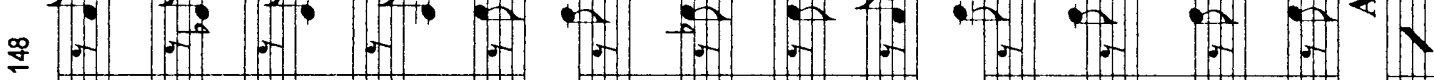

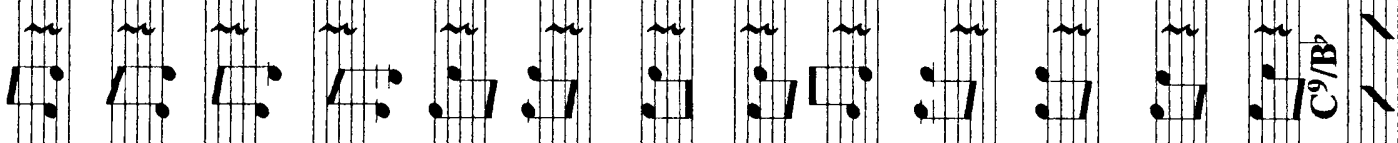

F.

1. I.

t.

$\stackrel{9}{q}$

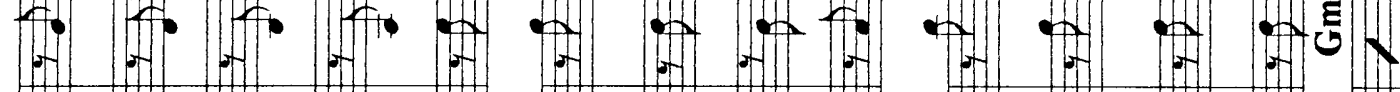

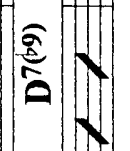

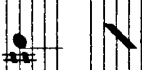

-

10

IN

$+1$

$1 \times$

- N

- $X$

$-N$

- N

a)

- N

- N

- $N$

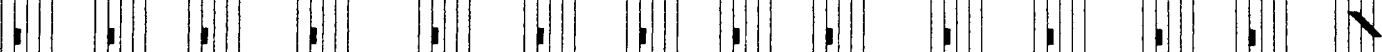

ב

-

- N

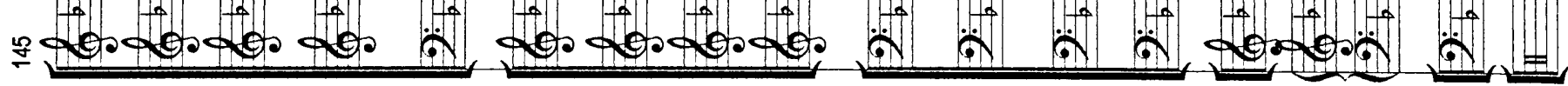



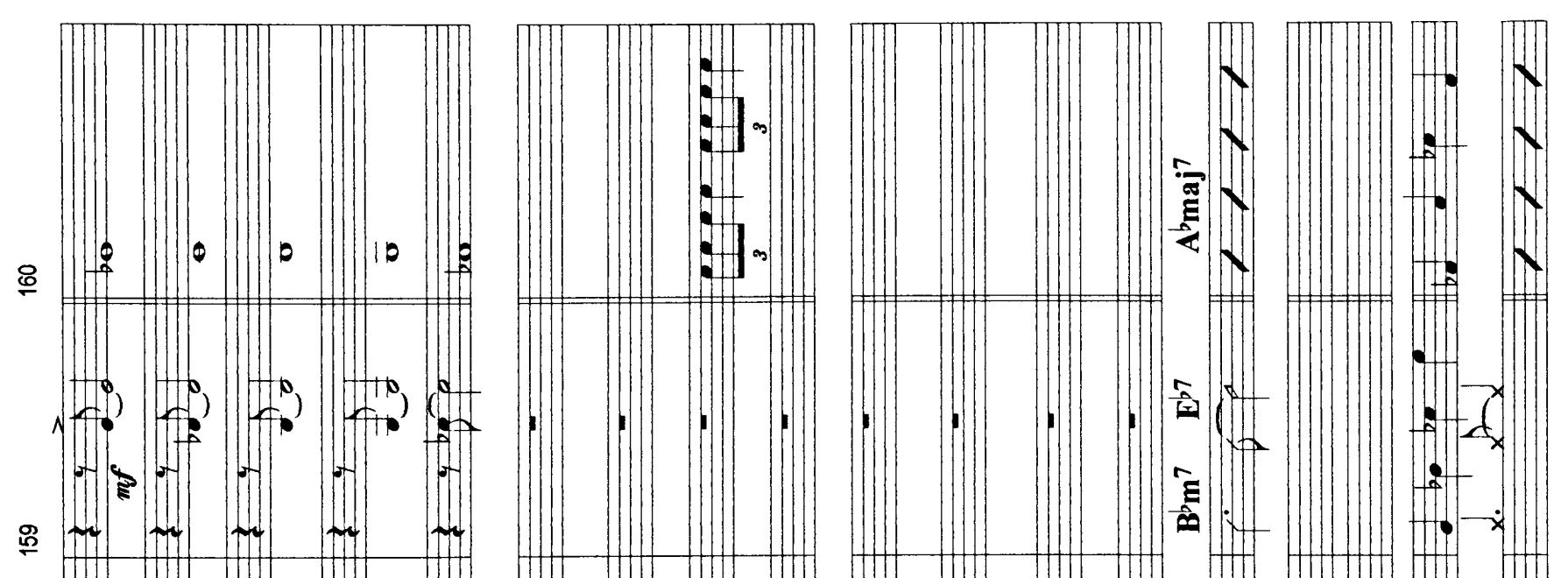

$\stackrel{\infty}{\stackrel{m}{\sim}}$

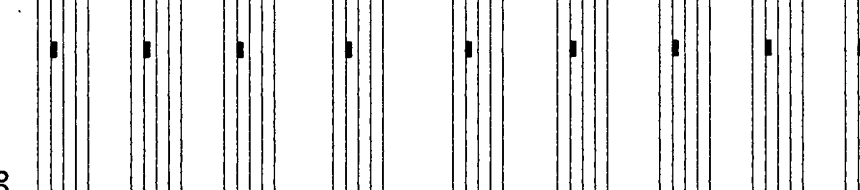

N.

$\because$. $:$ in $_{i}$

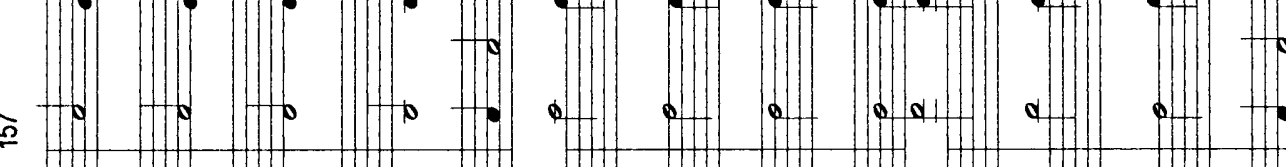

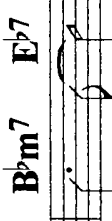

- $d_{x}^{x}$

:-

$\dot{x}$

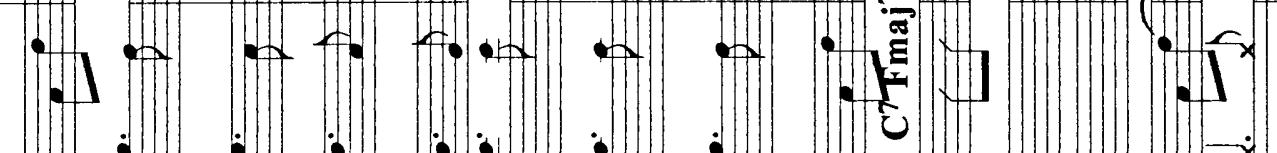

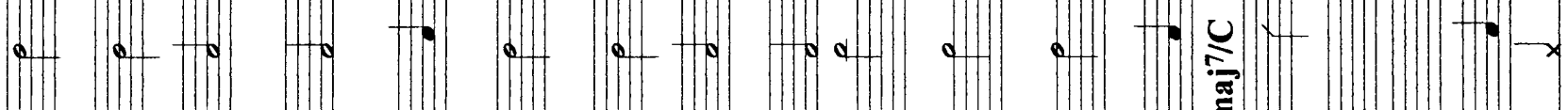

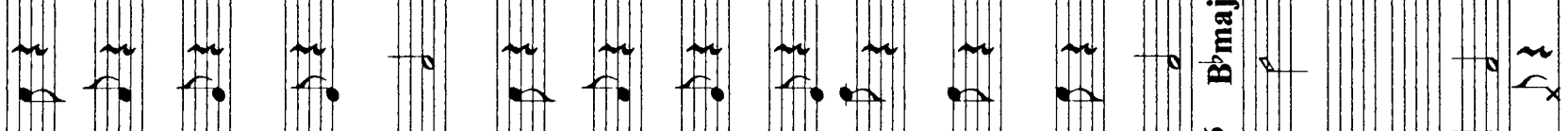

$\stackrel{\wp}{\leftrightarrows}$

an $\rightarrow$ m th

+ $\$$ -

总

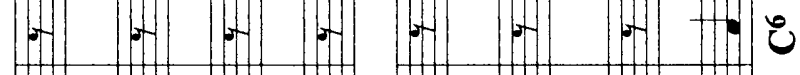

艾

$+\infty$

$+x$

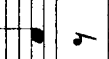

点

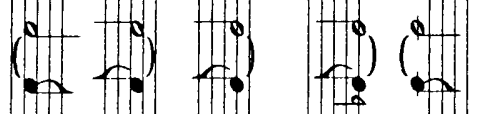

| || | || || || || || || || ||

$\sqrt{ }$

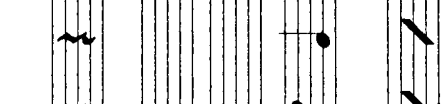

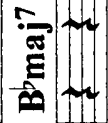

N

H

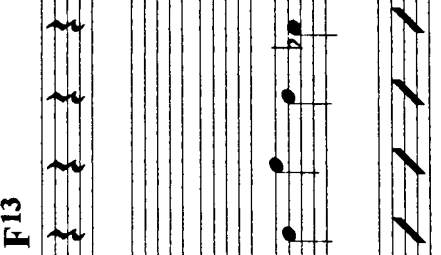

$\therefore \quad: \quad+0$ i

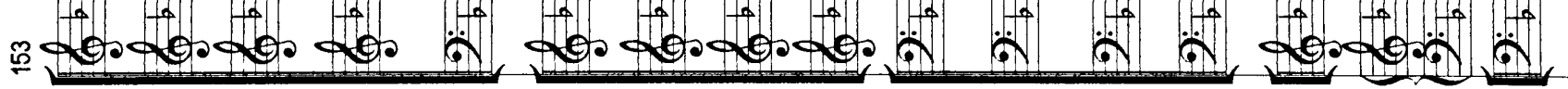




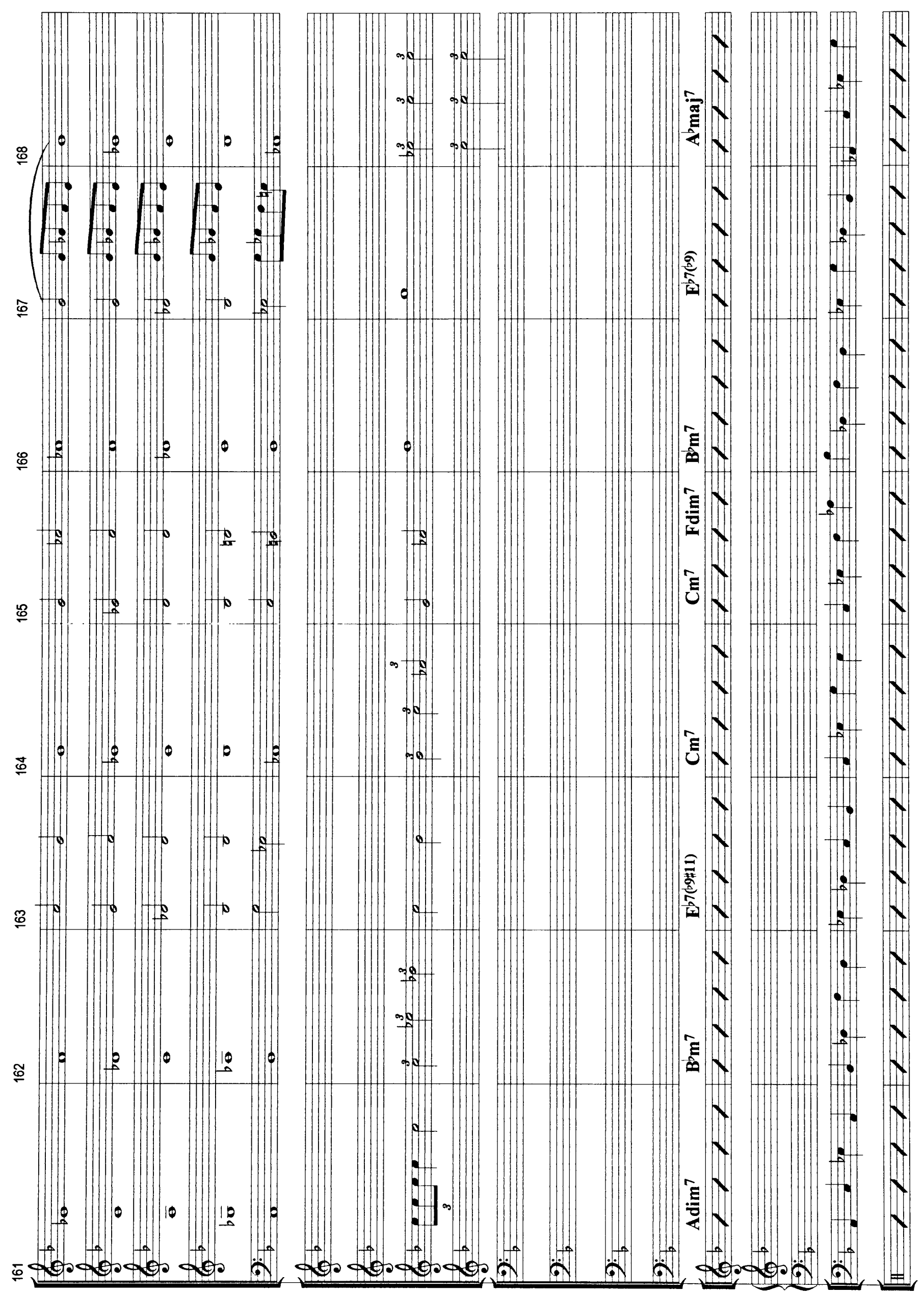


1:

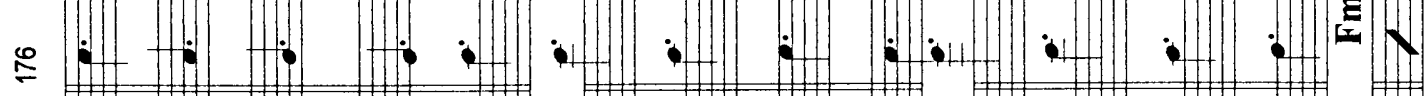

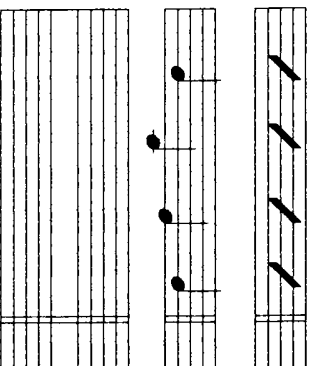

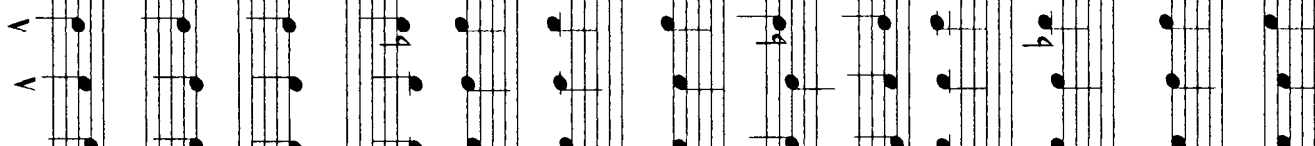

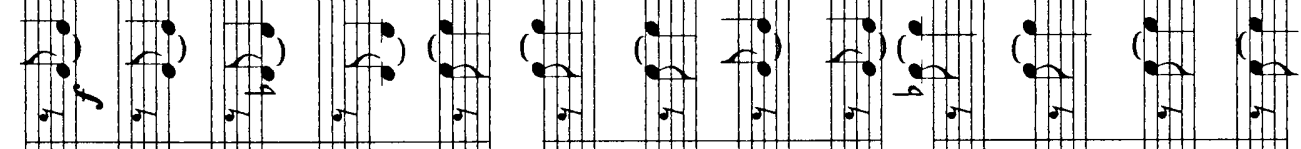

$\wedge \rightarrow N \rightarrow N \rightarrow \infty$

II $\ldots$

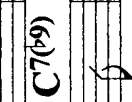

$\sqrt{x}$

$+\infty$

s

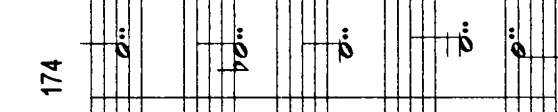

$$
\text { m. . . . . - m }
$$

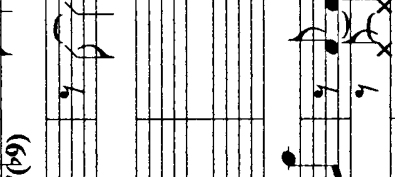

$\cong: 1: \div: 0$
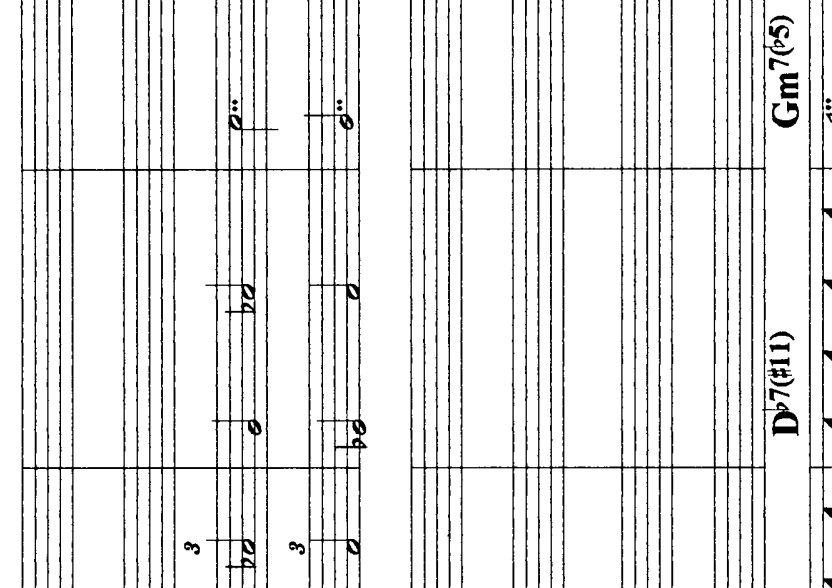

$\infty$

ล

$m a-\infty+0$
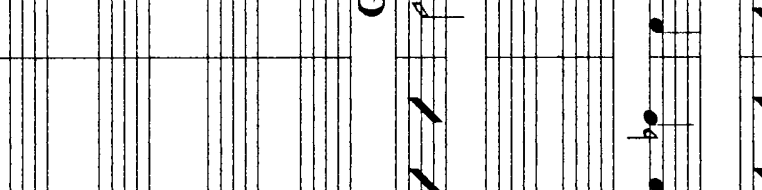

O Ho Ha

$\approx N+\infty \quad+\infty$

a. +0

(1)

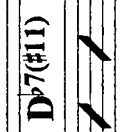

30 mo

E

에

$\infty 01)$

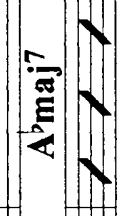

$\sqrt{1}$

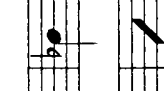

-

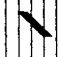

$\sqrt{2}$ 
of of

क्ष

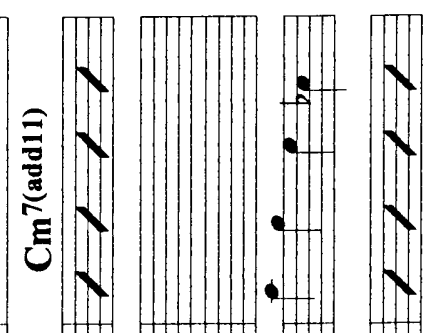

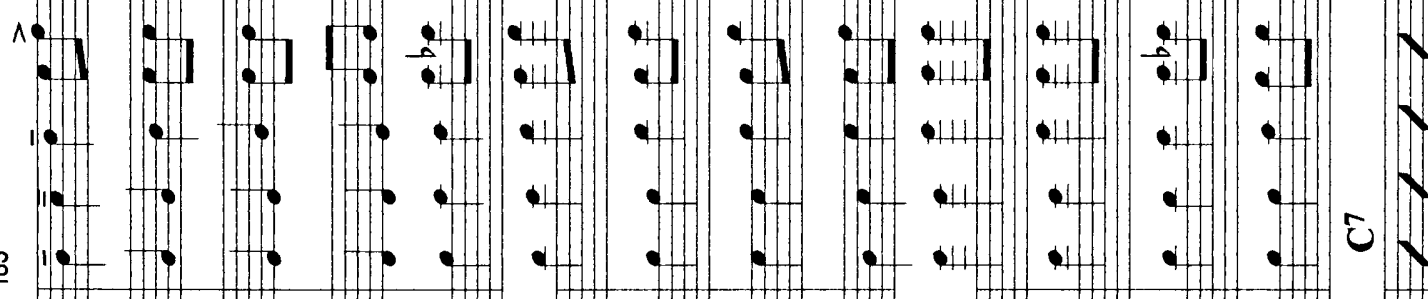

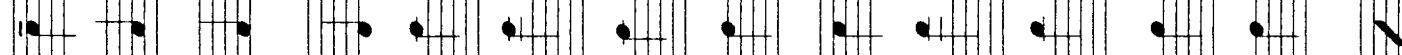

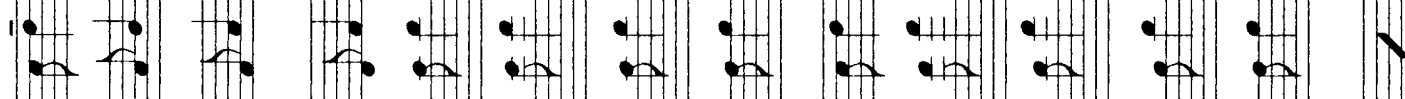

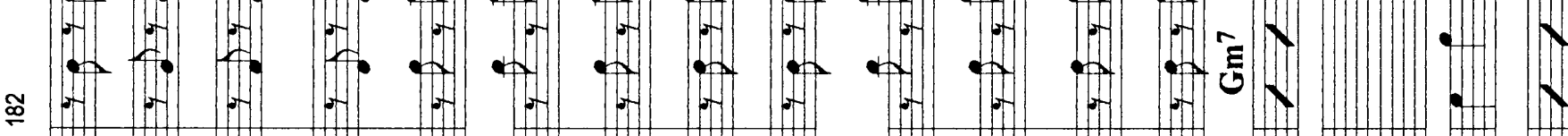

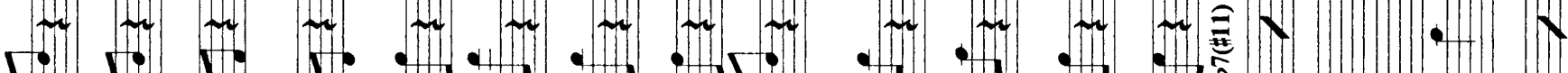

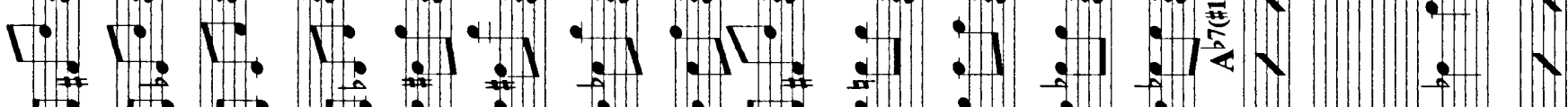

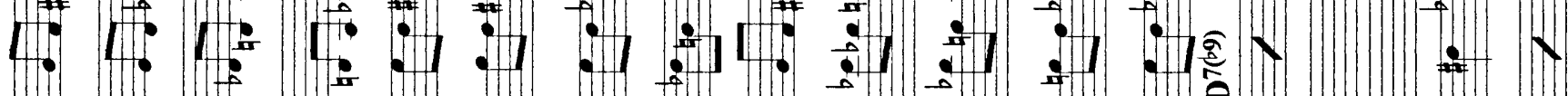

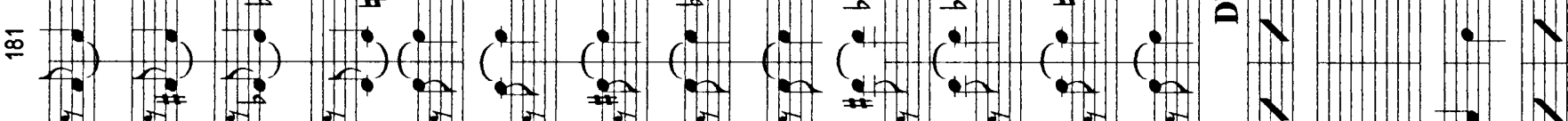

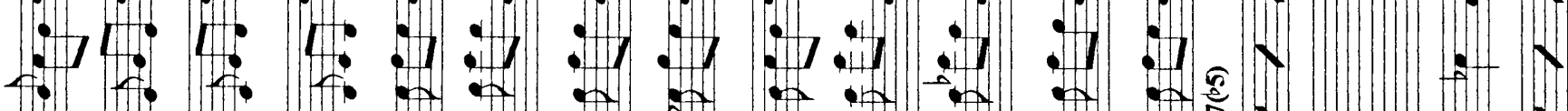

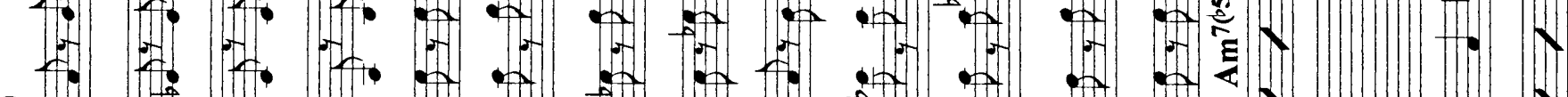

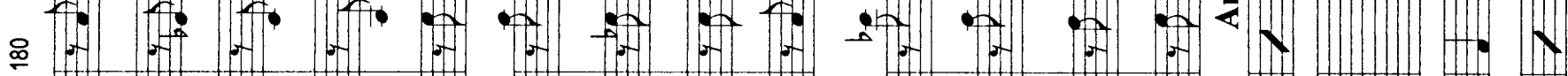

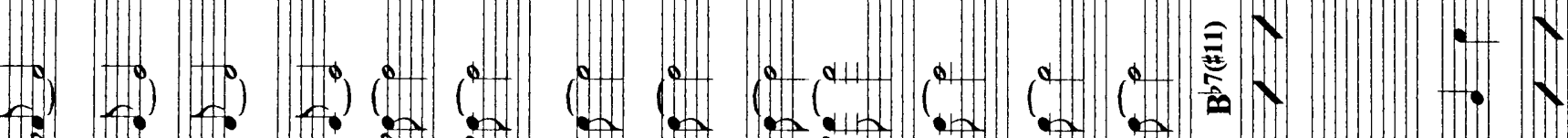

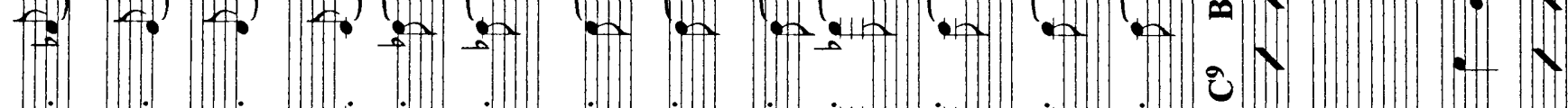

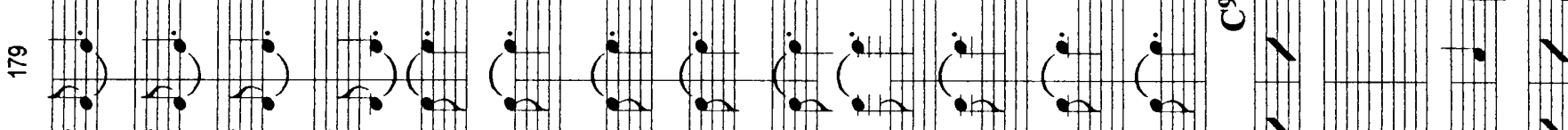

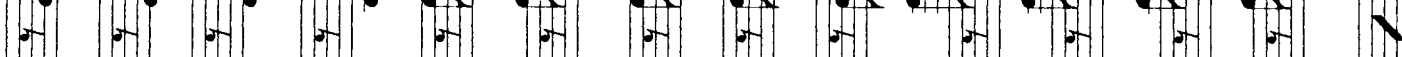

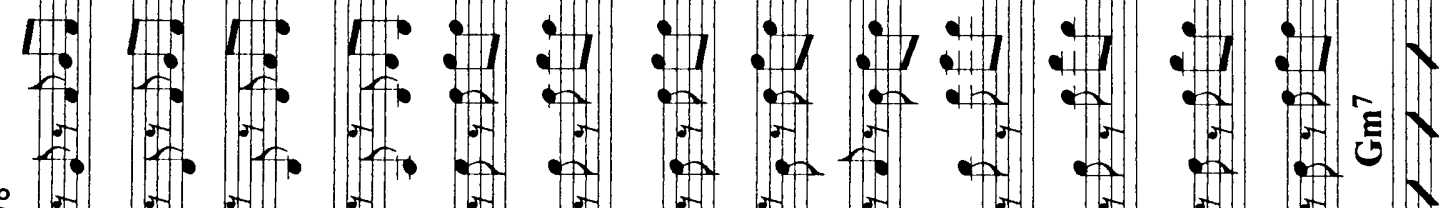

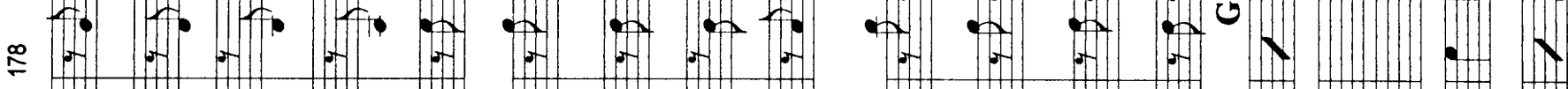

Let

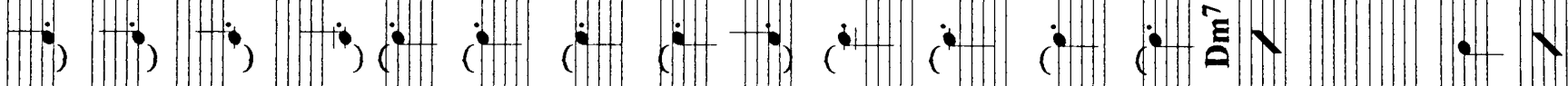

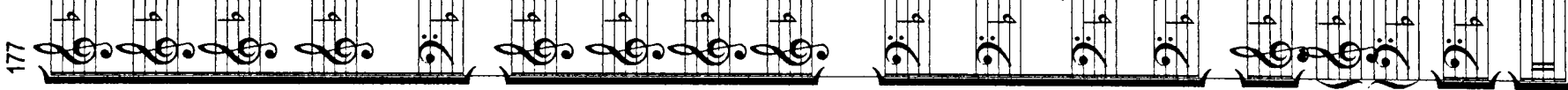


<.

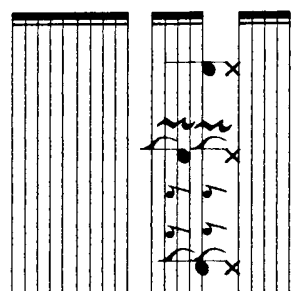

Fin

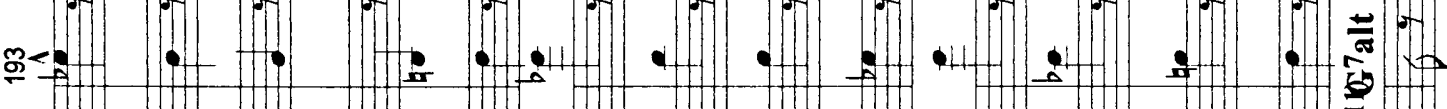

$f 6 x$

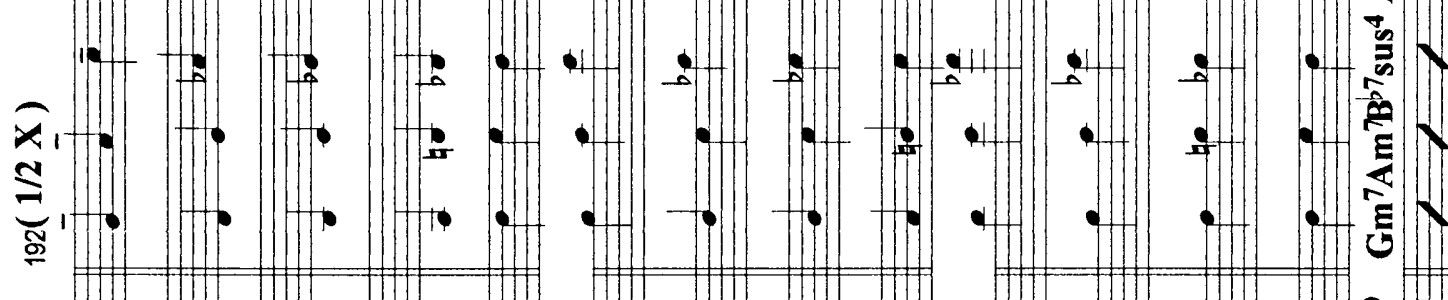

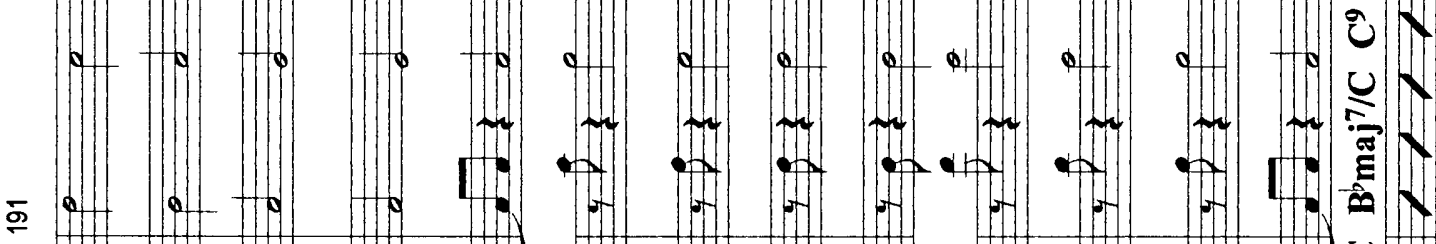

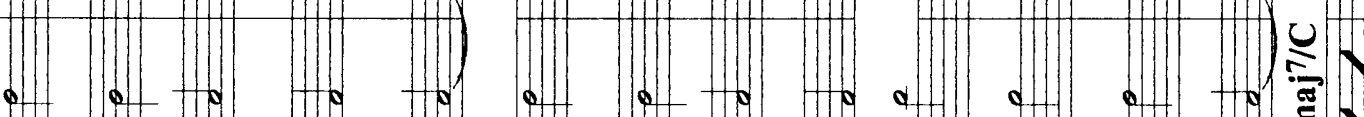

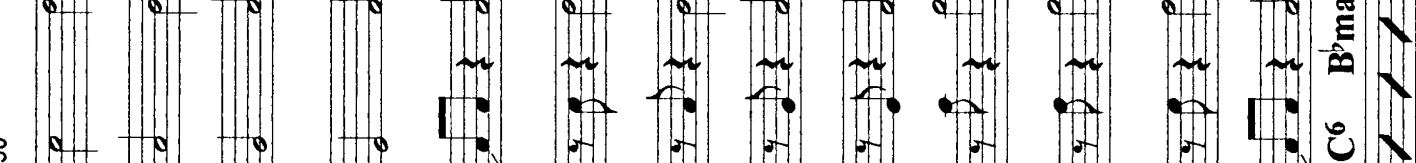

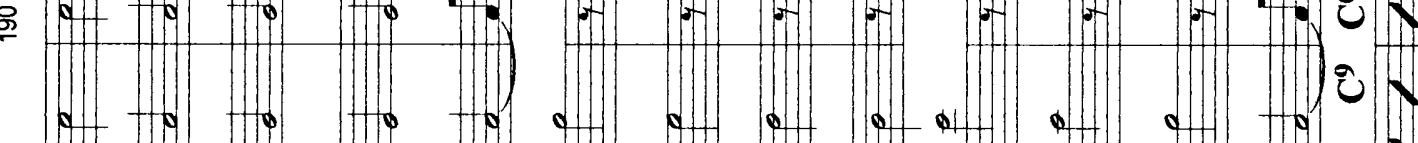

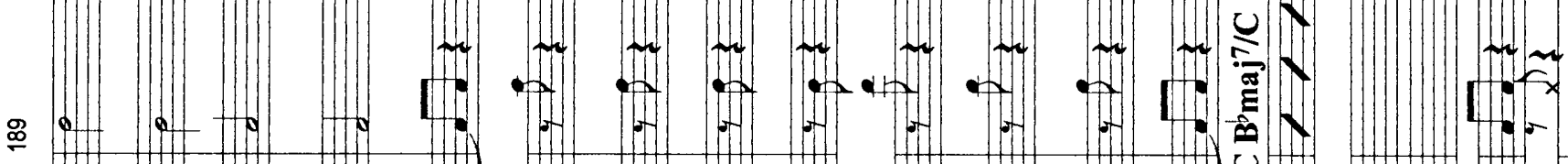

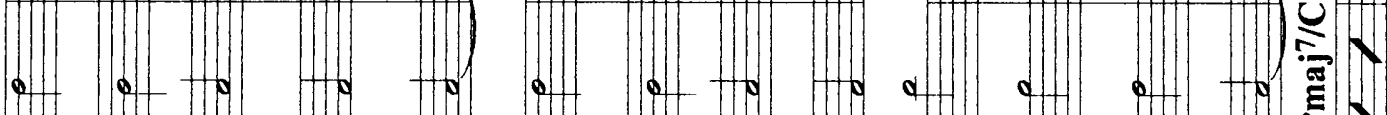

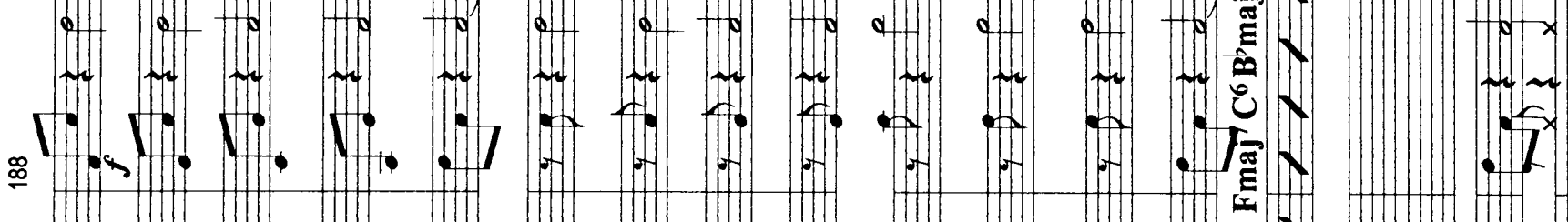

Ho.

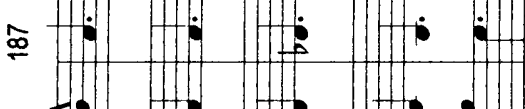

ton

(2)

- H: H: H:

ind

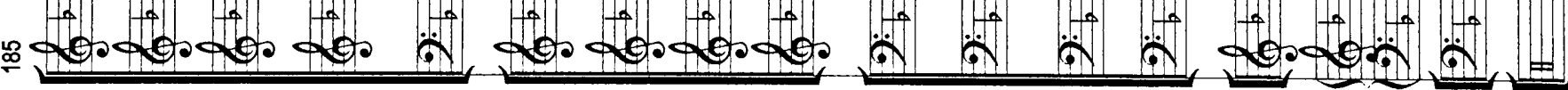



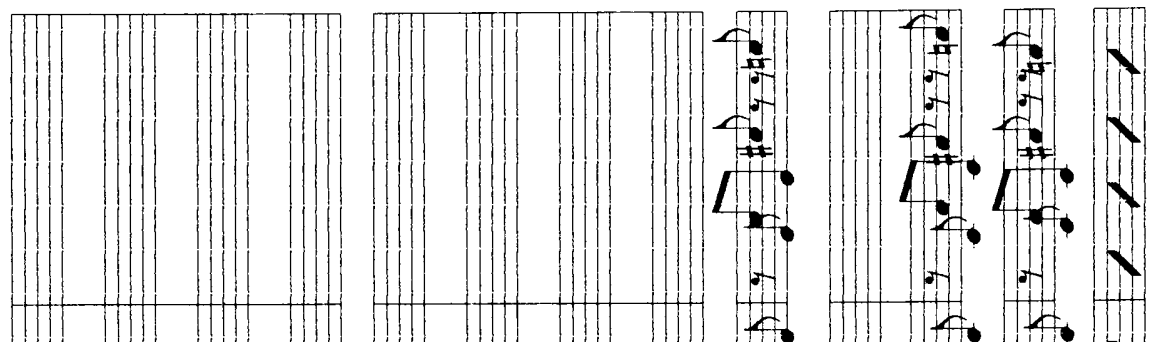

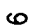
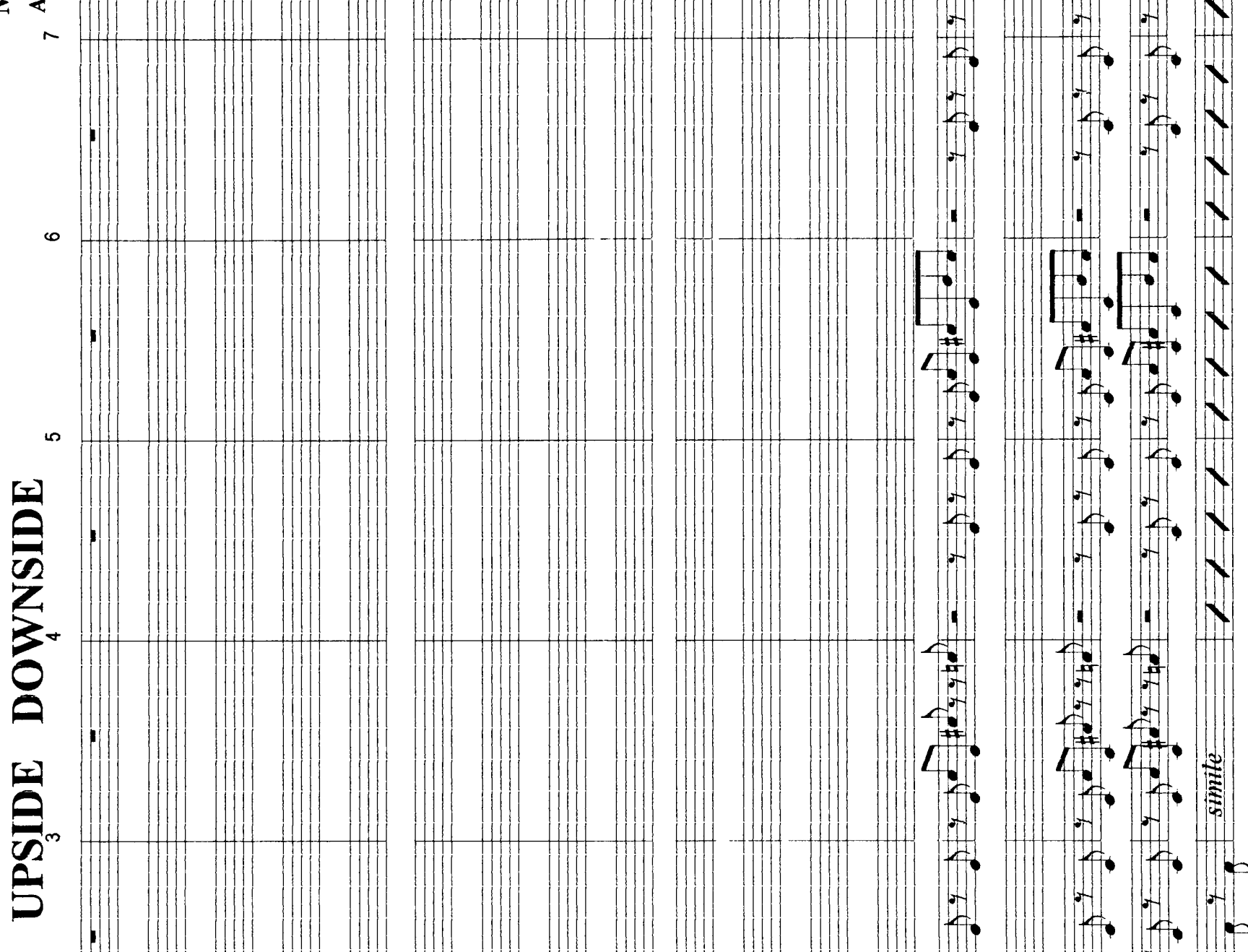

is
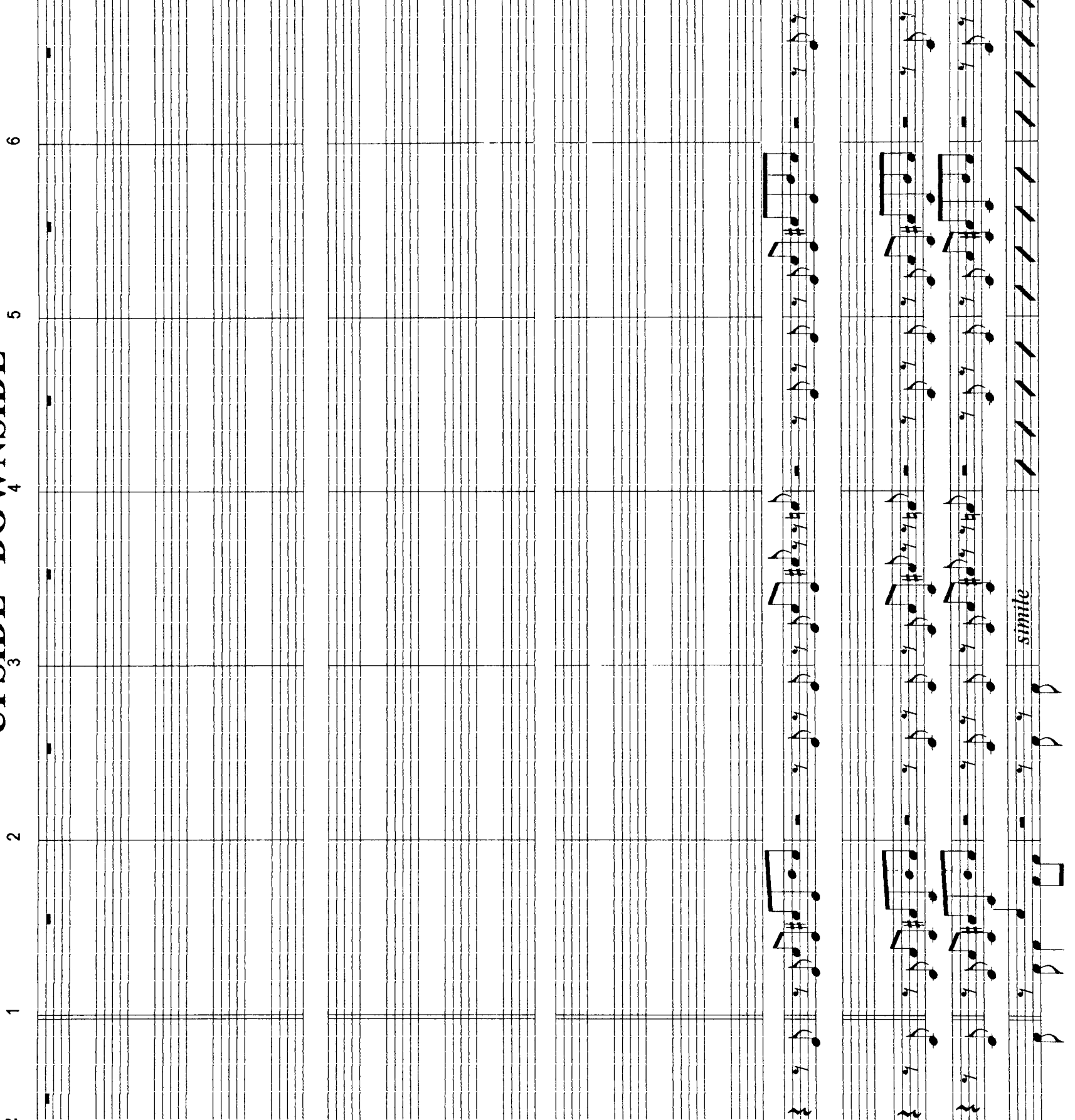

ֻั

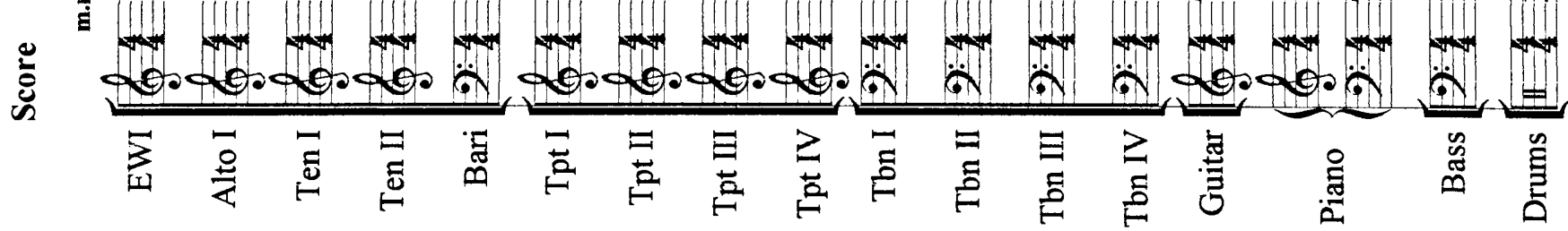




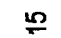

$\exists$

m

$\simeq$

우

,

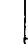

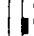

$\infty$

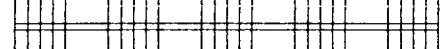

,

+ 1.1 .1$.

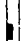

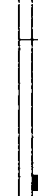

1)

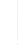

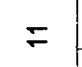

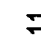

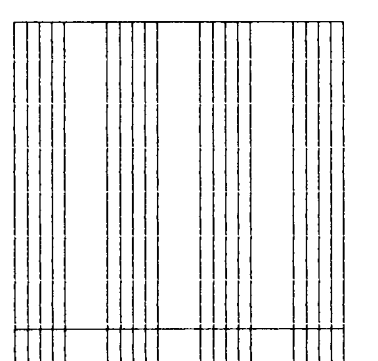

\|\|$\|$
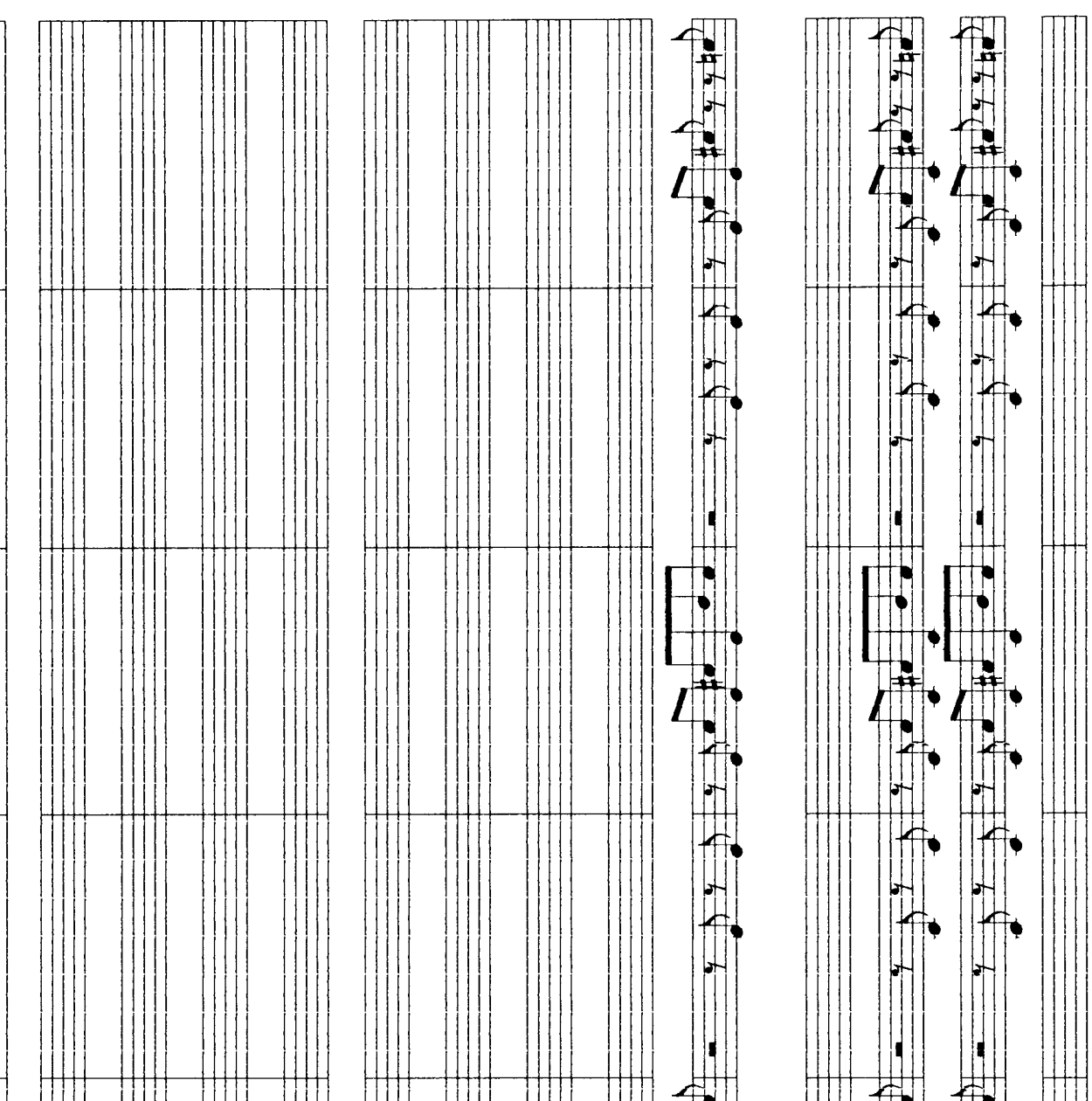

4

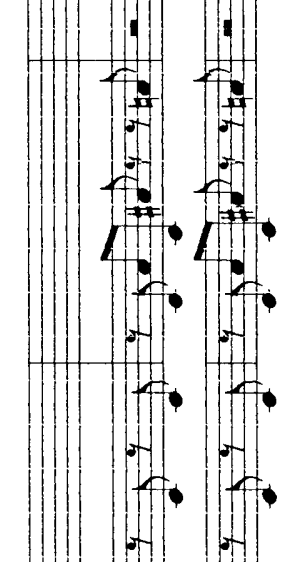



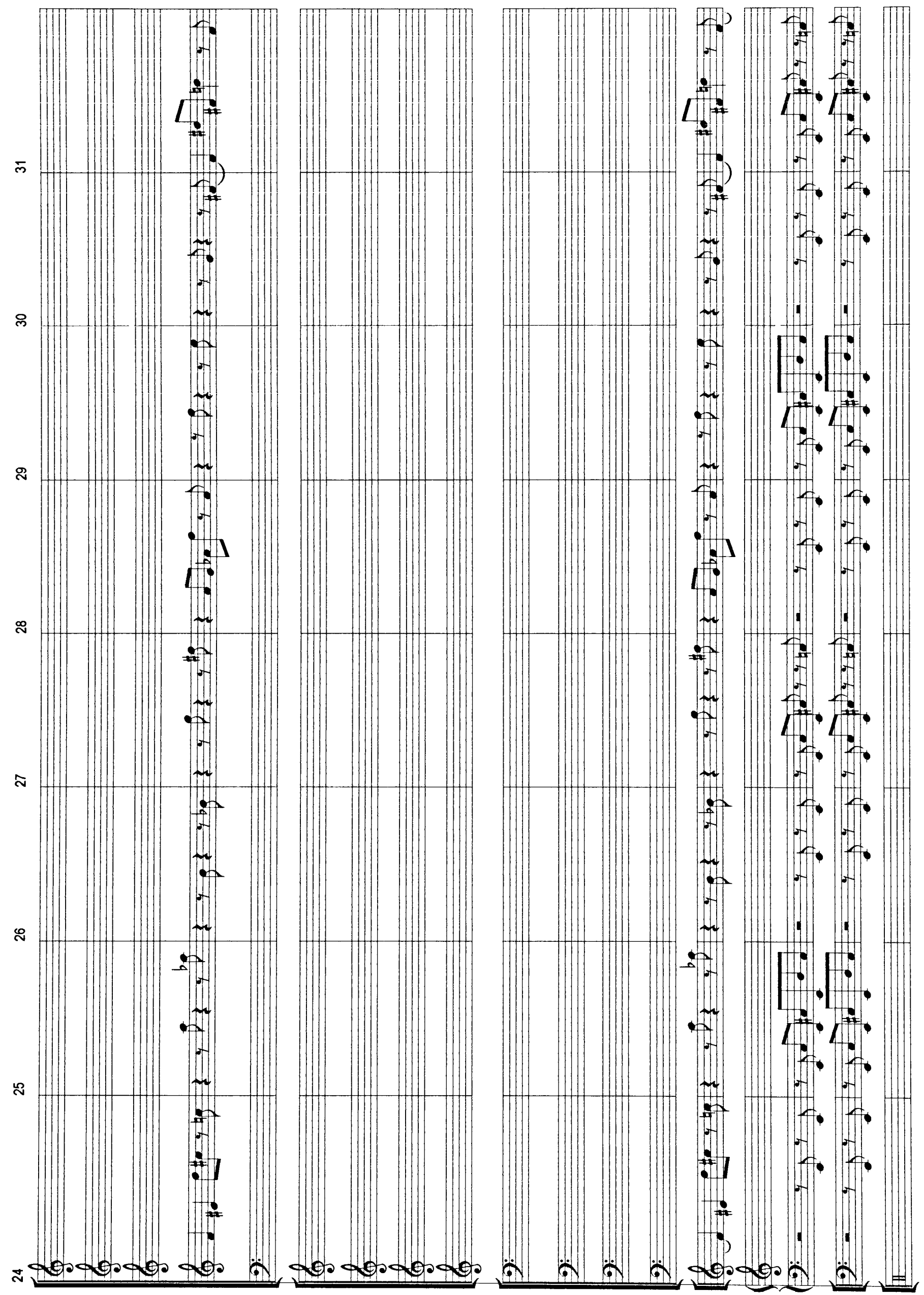


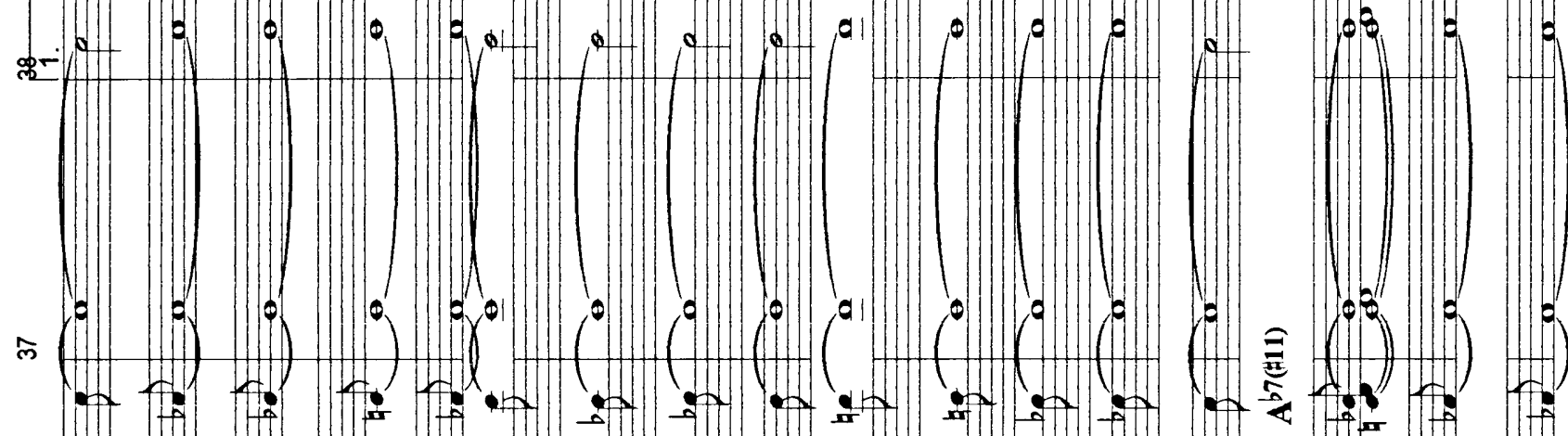

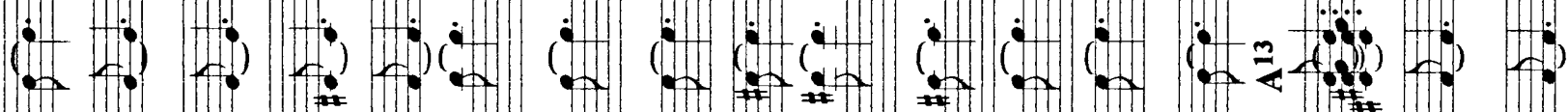
c.

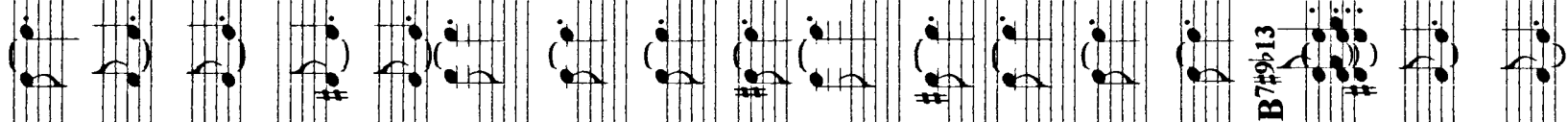
$\left(\begin{array}{l}(1) \\ 0\end{array}\right.$

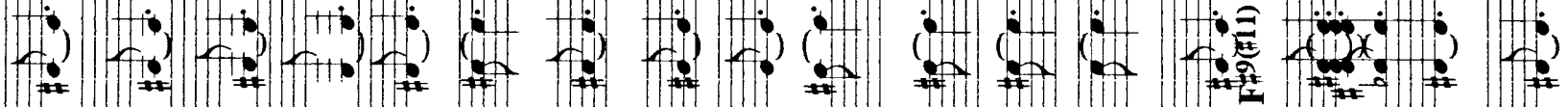
z (1) H. Ho

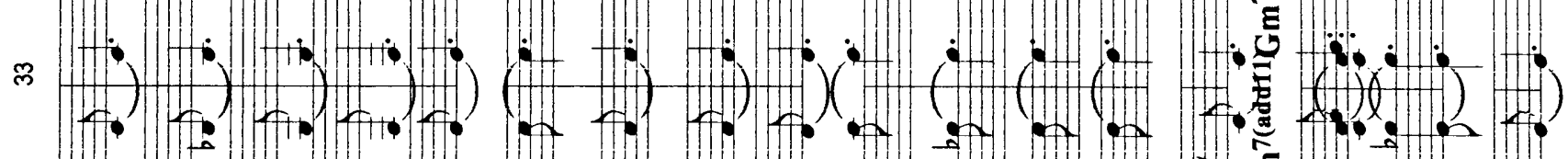

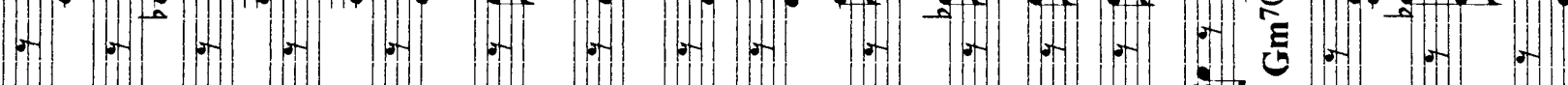

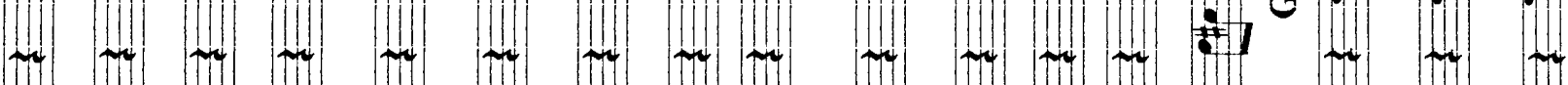
- III

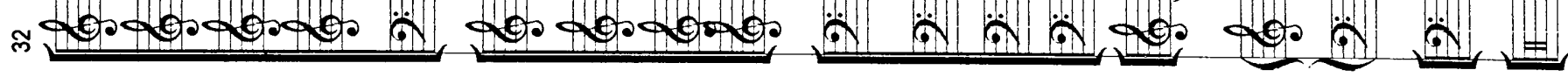



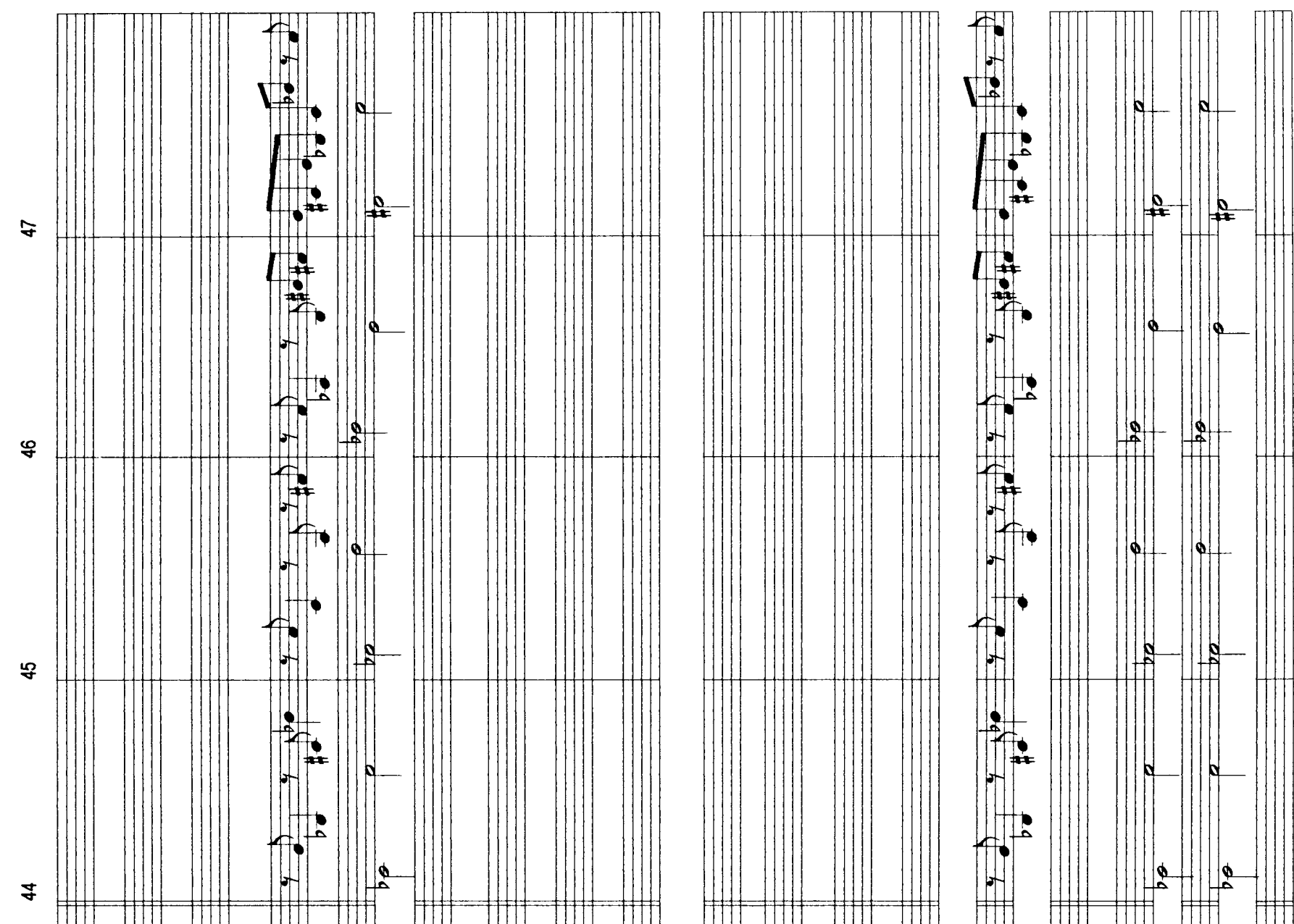

W. 30 H

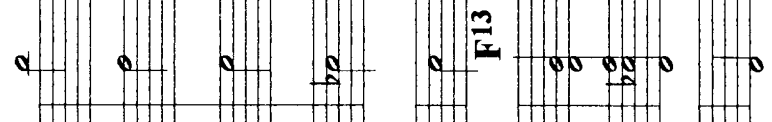

ษ

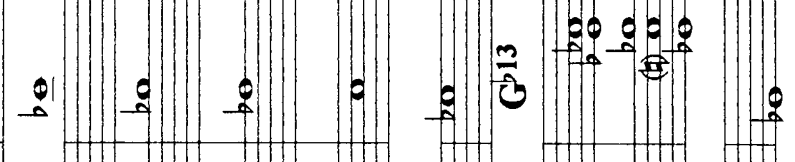

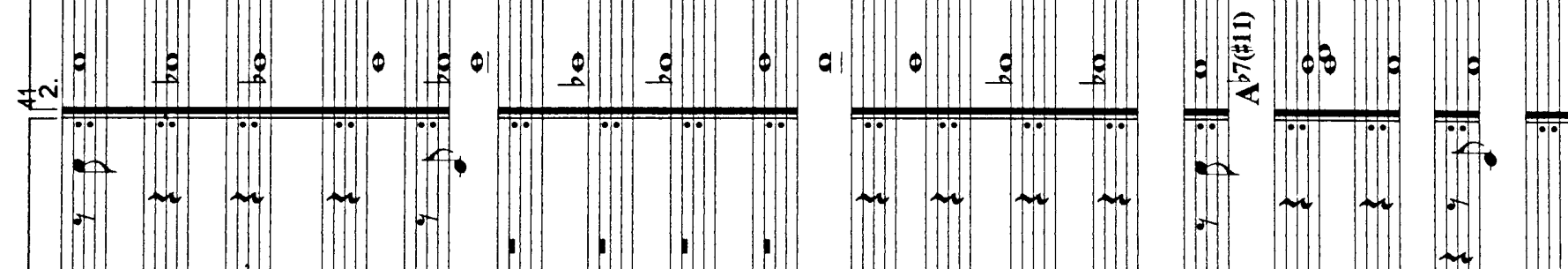

Hi.

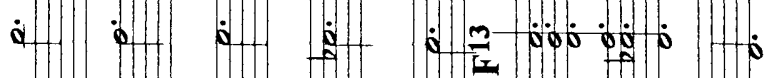

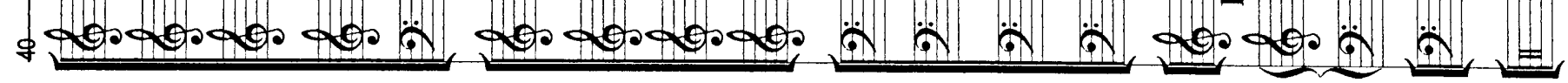




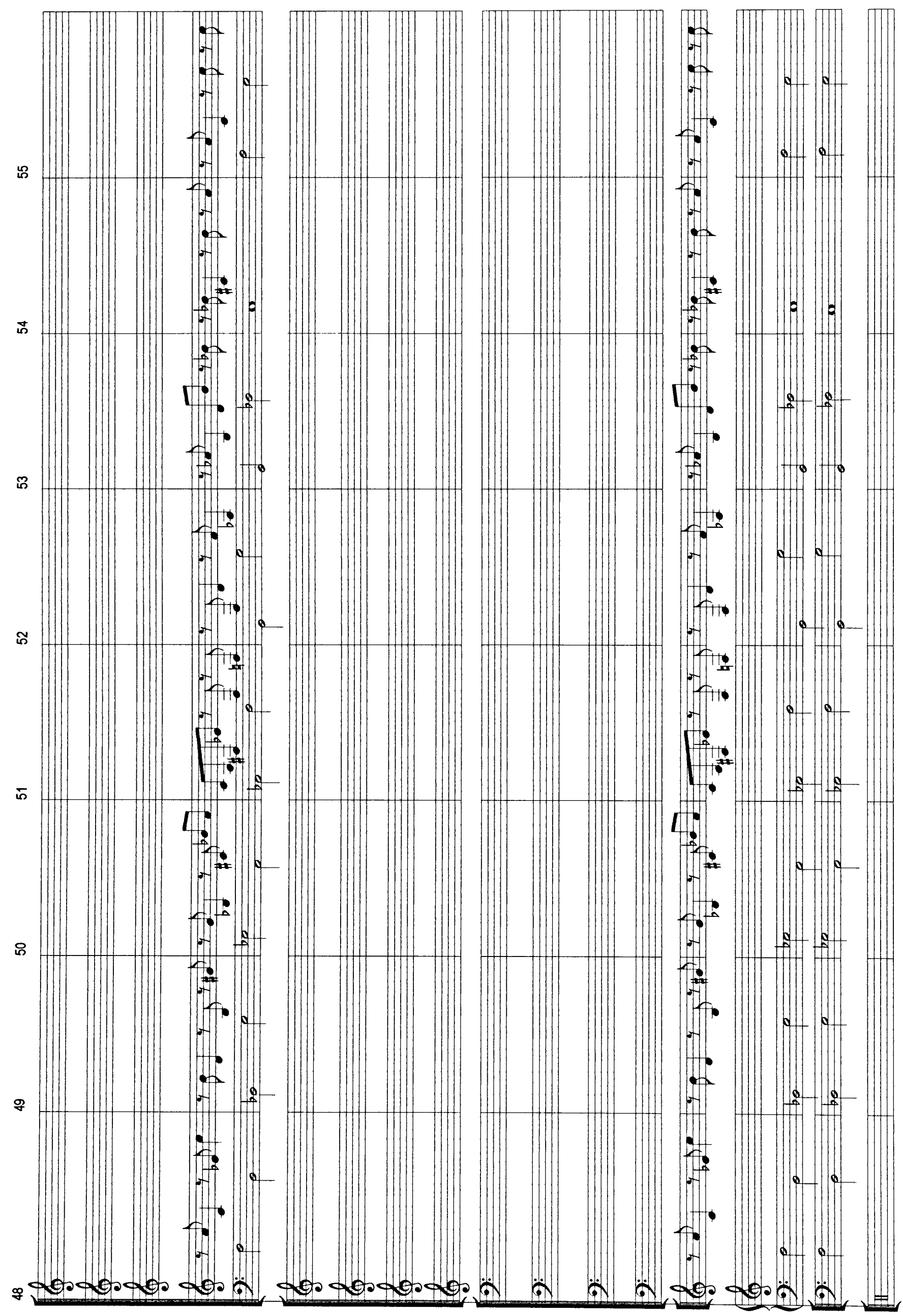




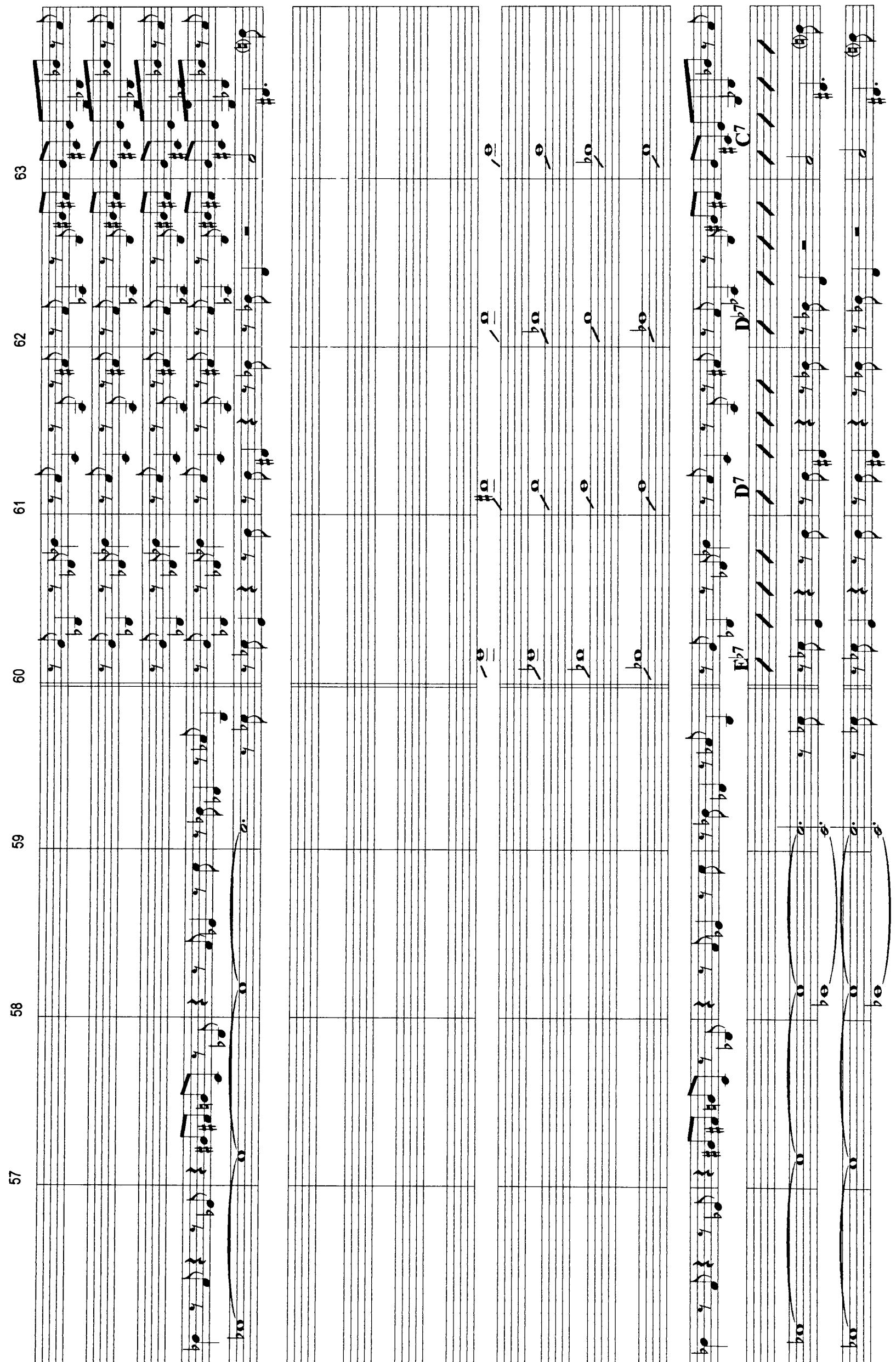

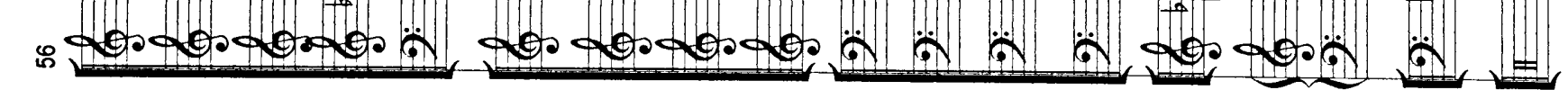




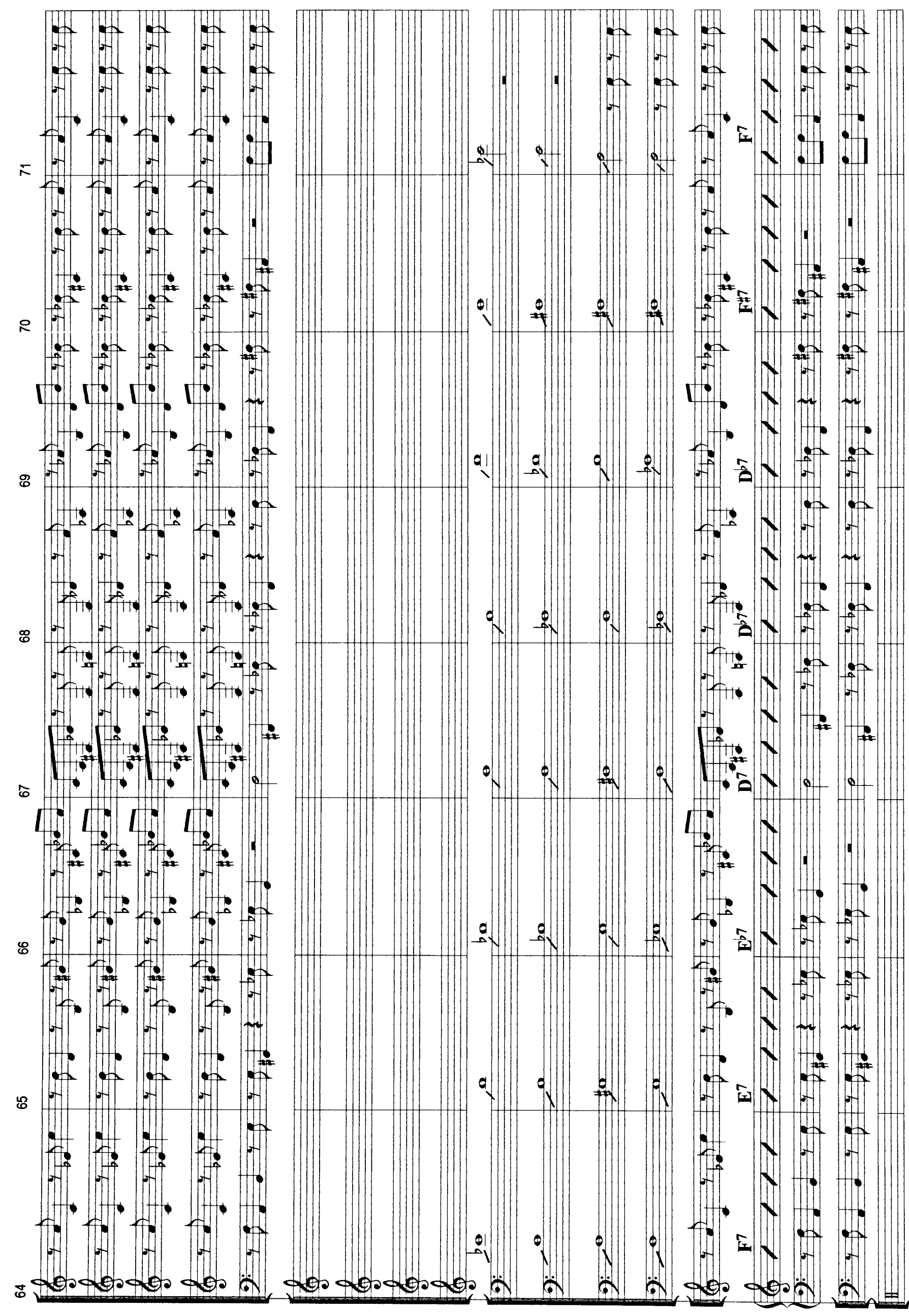




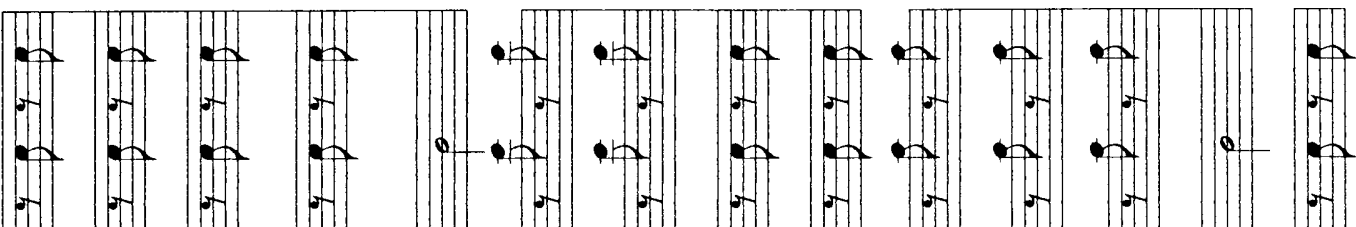

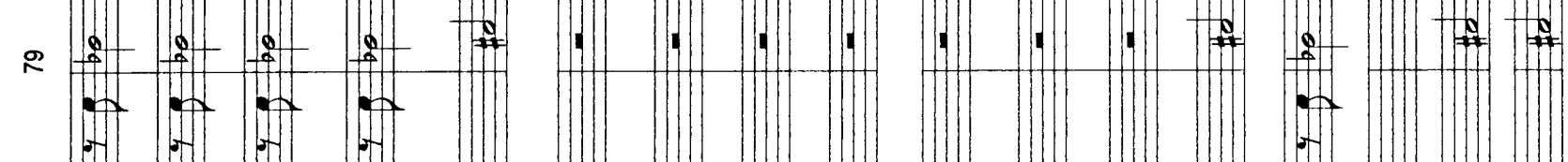

$\prod_{\infty}: \frac{1}{2}$

$\prod_{-\infty}^{\infty}$

$\lim _{\rightarrow \rightarrow-\infty} \rightarrow-$

1.1.1. 1.0

$\because \div$

$\therefore$ T)

H.

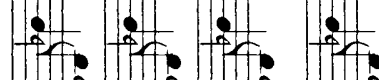

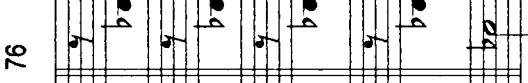

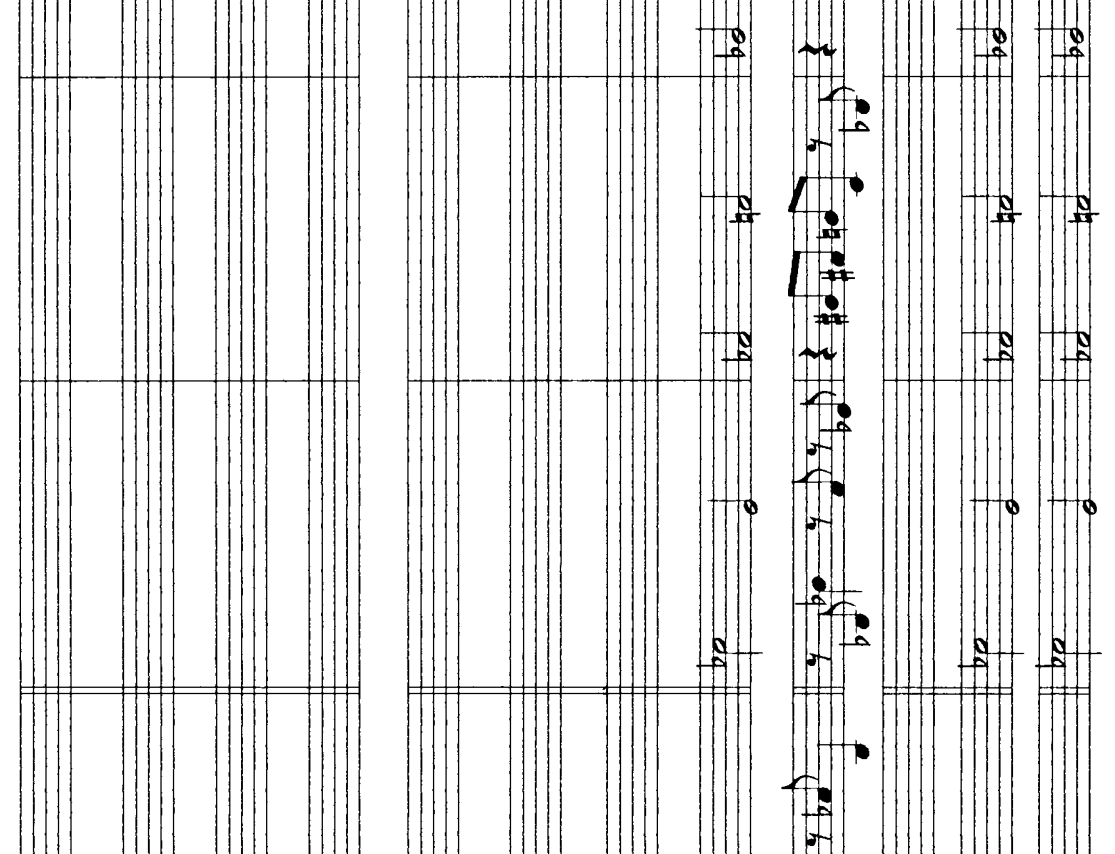

Hat

II $+\frac{8}{2}+0$

$\sum_{i=1}^{\infty}: \infty$

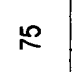

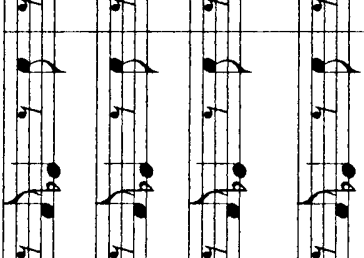

$\therefore$ a do do

$\$$ 计

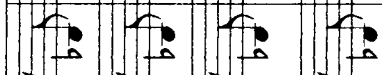

H. 1.10

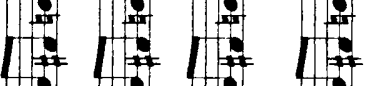

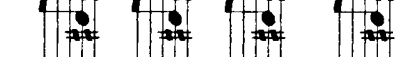
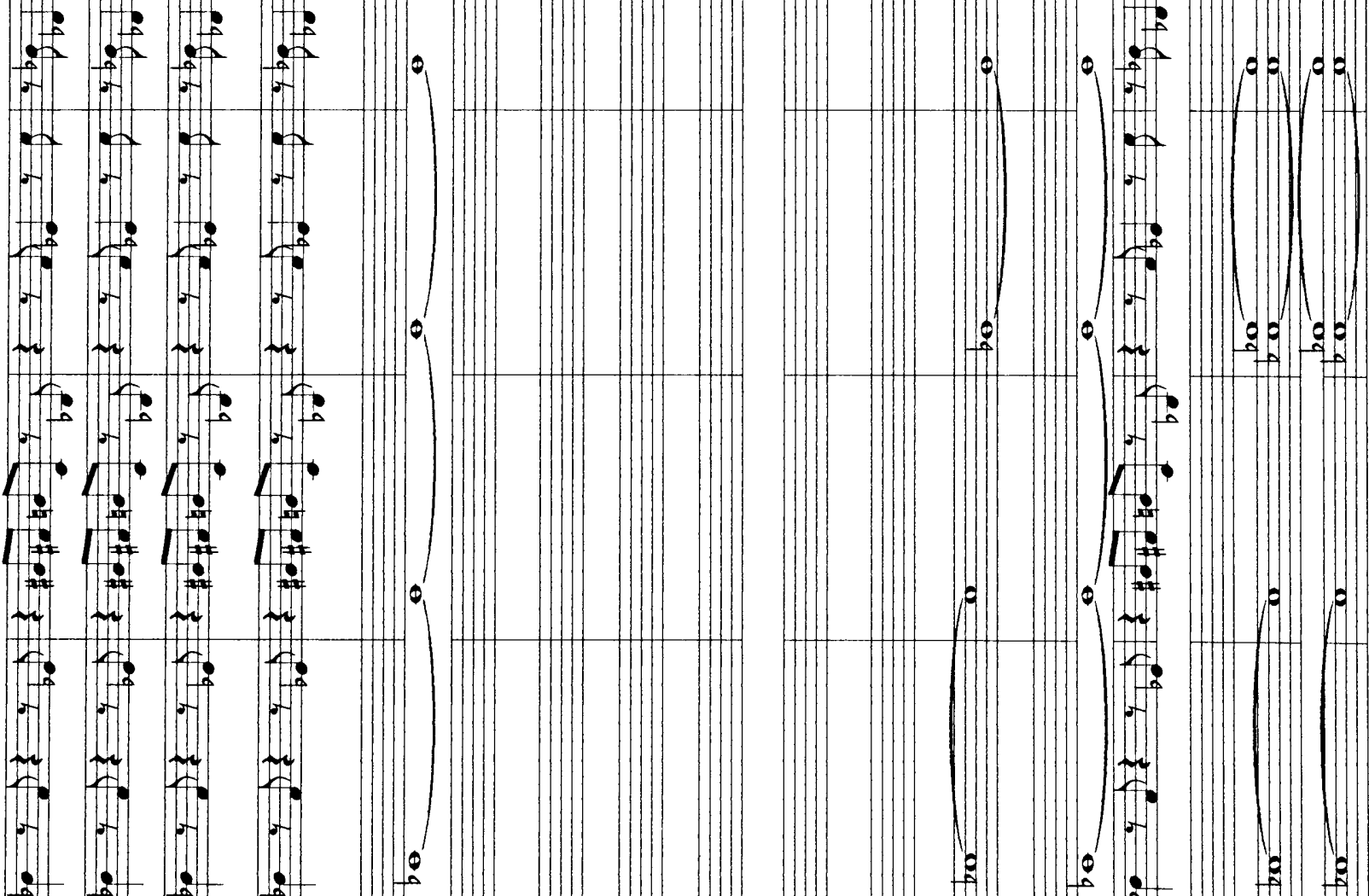

: :-1:- :

$\because \quad 6:-5$

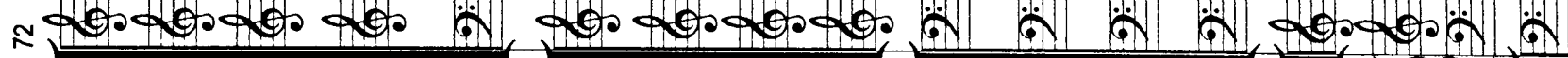




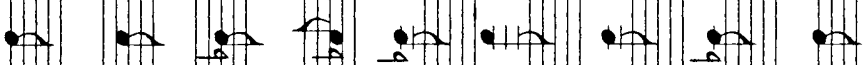

-

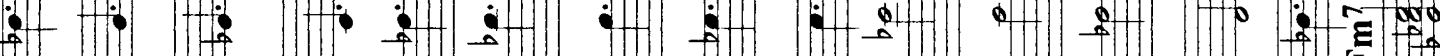

$\hat{\infty}$

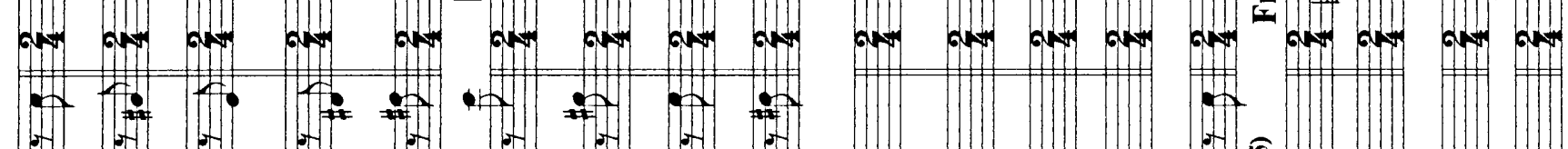

H:

\&

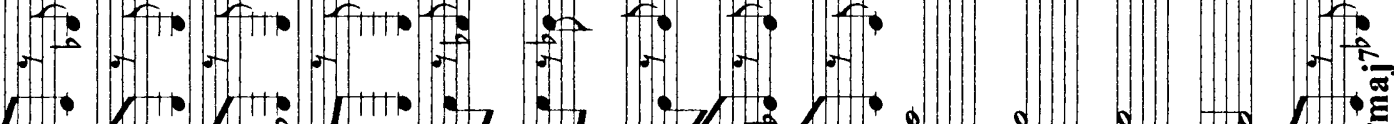

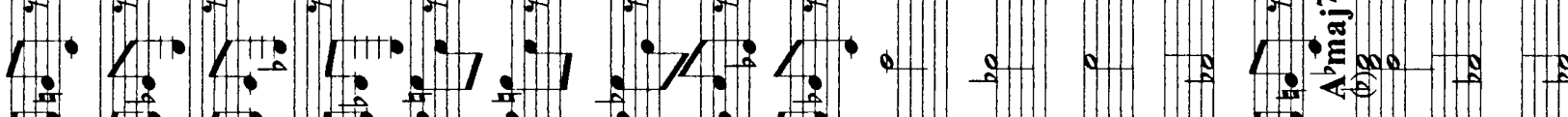

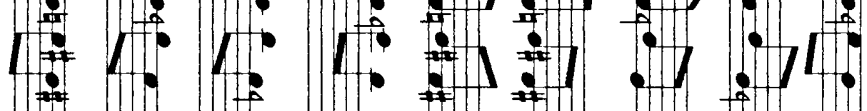

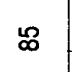

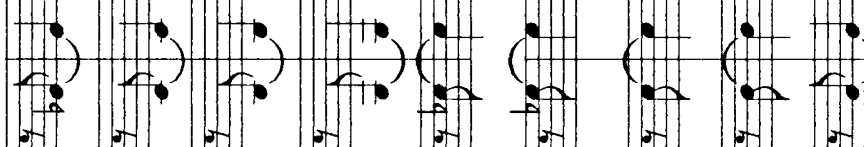

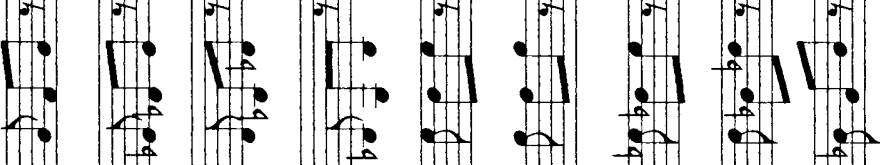
$\rightarrow$ a. $1+10$

:

Ë. E)

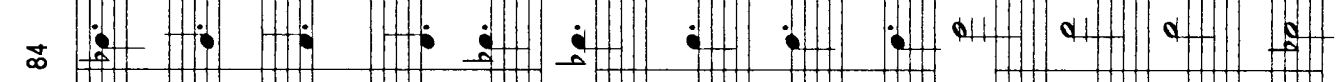

(1)

2.

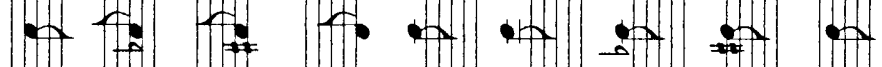

- $4000+2$.

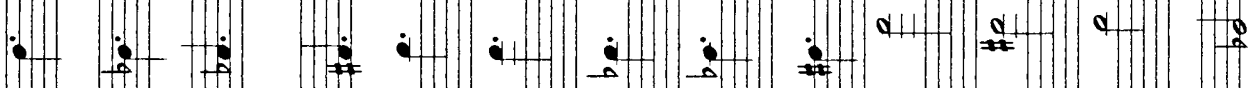

$\alpha \cos$

- 1 .
-. 11.
$\therefore$ \& 1.10
a + f
a. 011 .
dil)

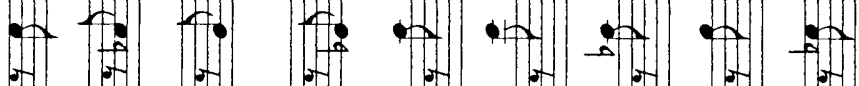

促:

×-4

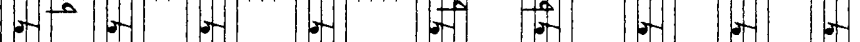

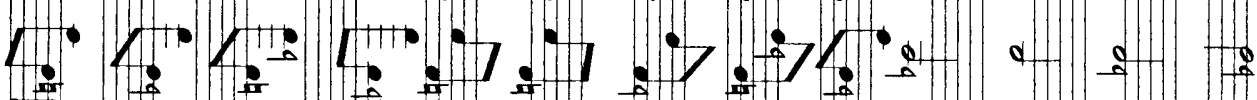

$\left.\because\right|_{0} ^{\infty}$

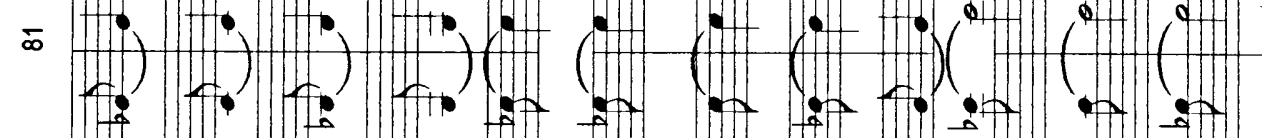

2.

10

(1)

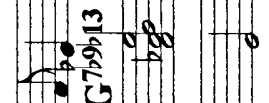

10

- $\rightarrow$ बे

4 E. Ed

a)

$1: 500$

a

Eे

H.

$\operatorname{lom} \frac{0}{5}$

$\therefore$ - 10 -

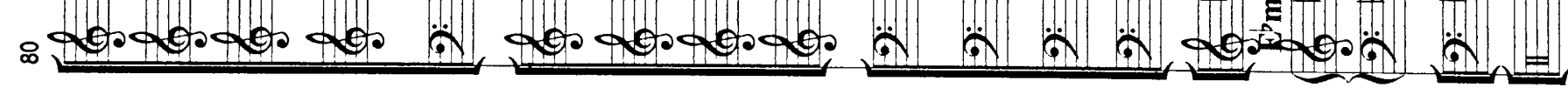




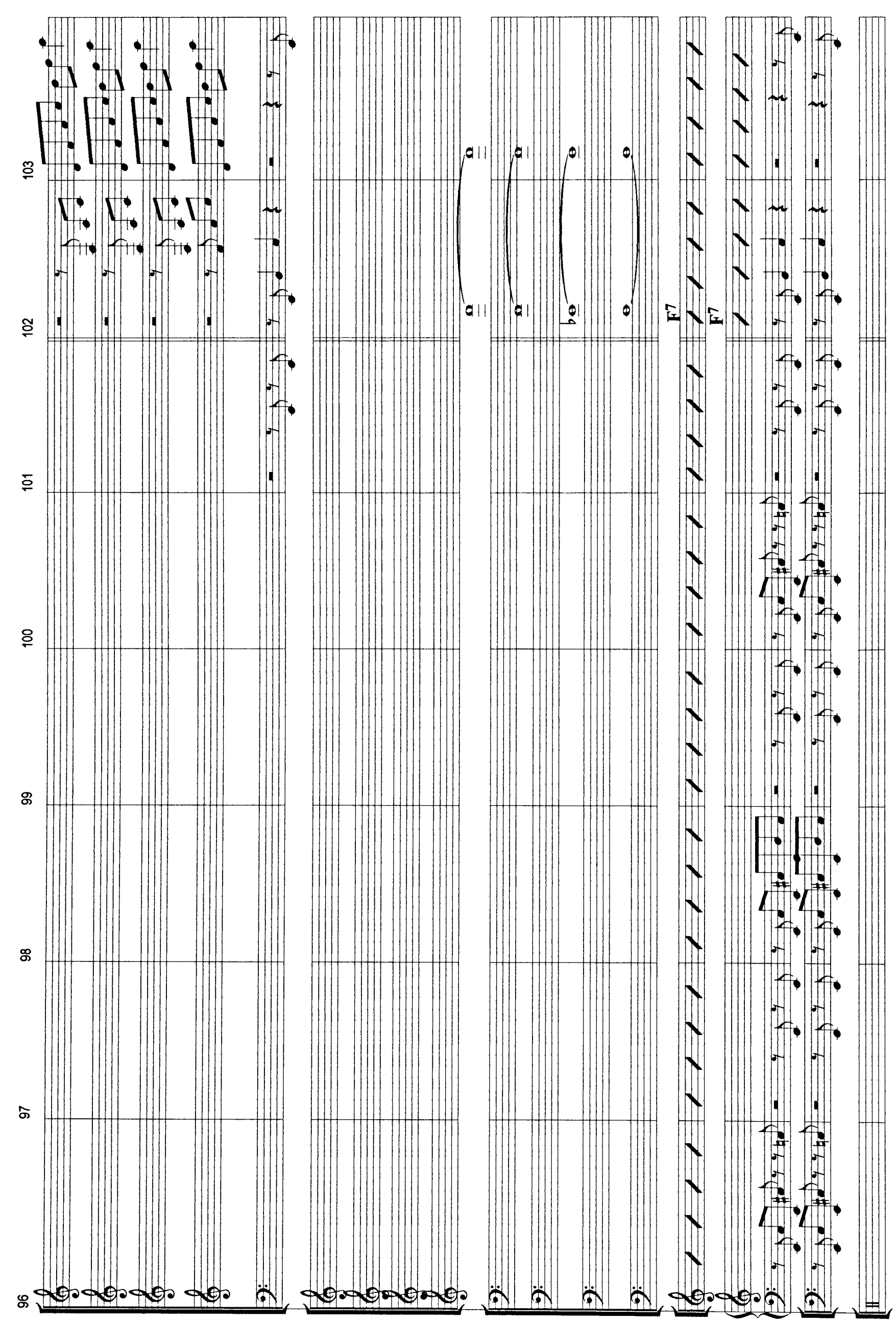


:

o: $0^{\circ} \cdot i^{\circ}$

$\stackrel{0}{g}=0$.

$: 1: 1: 1=1$

::: :: ::: ::

$1: 1: 1: \div$

H

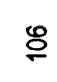

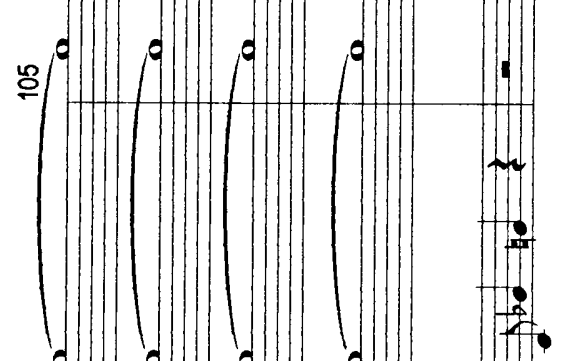

$\therefore$ :

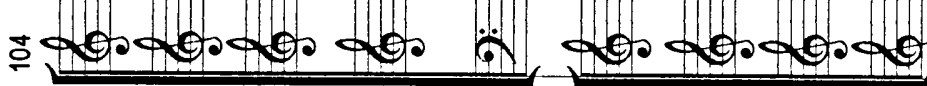

a). .12

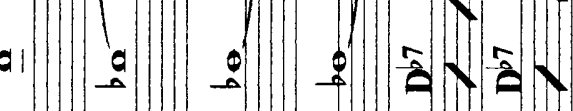

$:$

$+\infty$

$x+\ldots$

$>-\infty$

$x \sqrt{ }$

a) a $9 . \mid-\infty \times$

a) . D >

ax: $=x$

$x+\infty$

$\gamma \times$

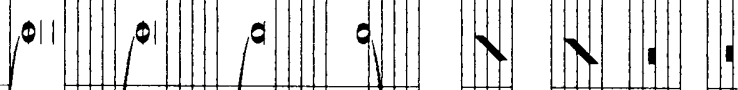

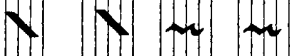

$X=:$

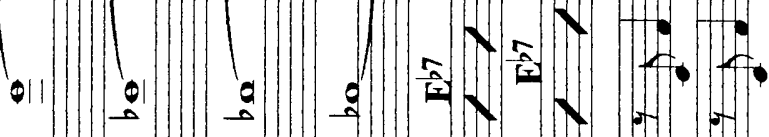
.

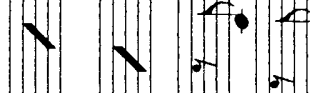

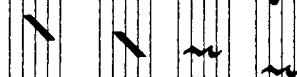

$\gamma$

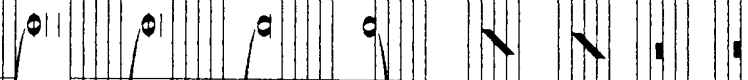

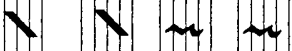

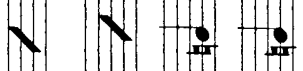

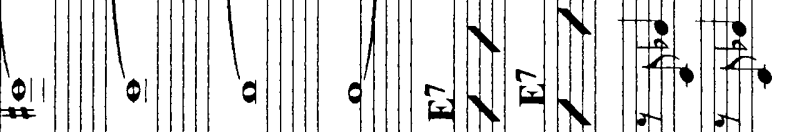



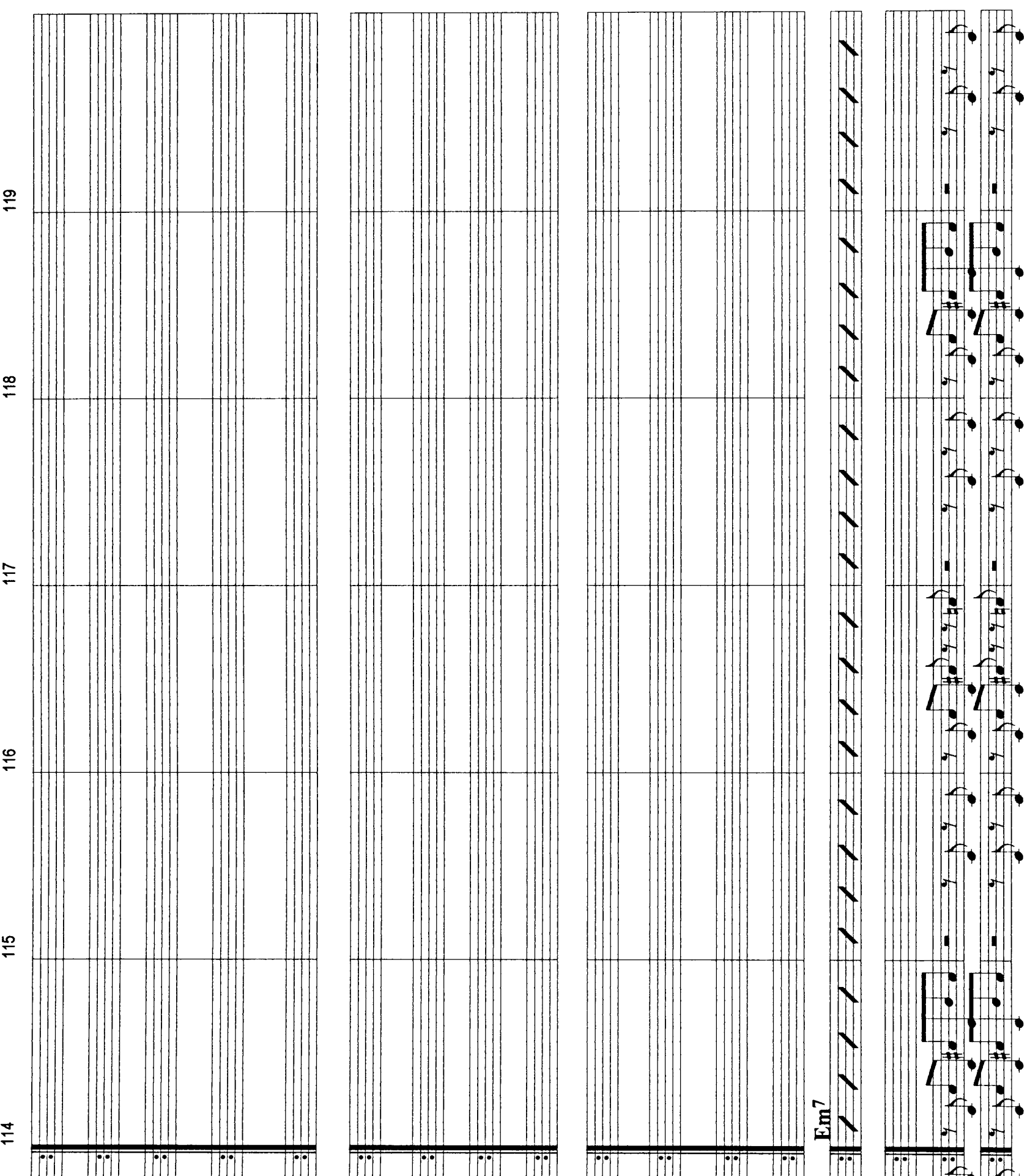

I
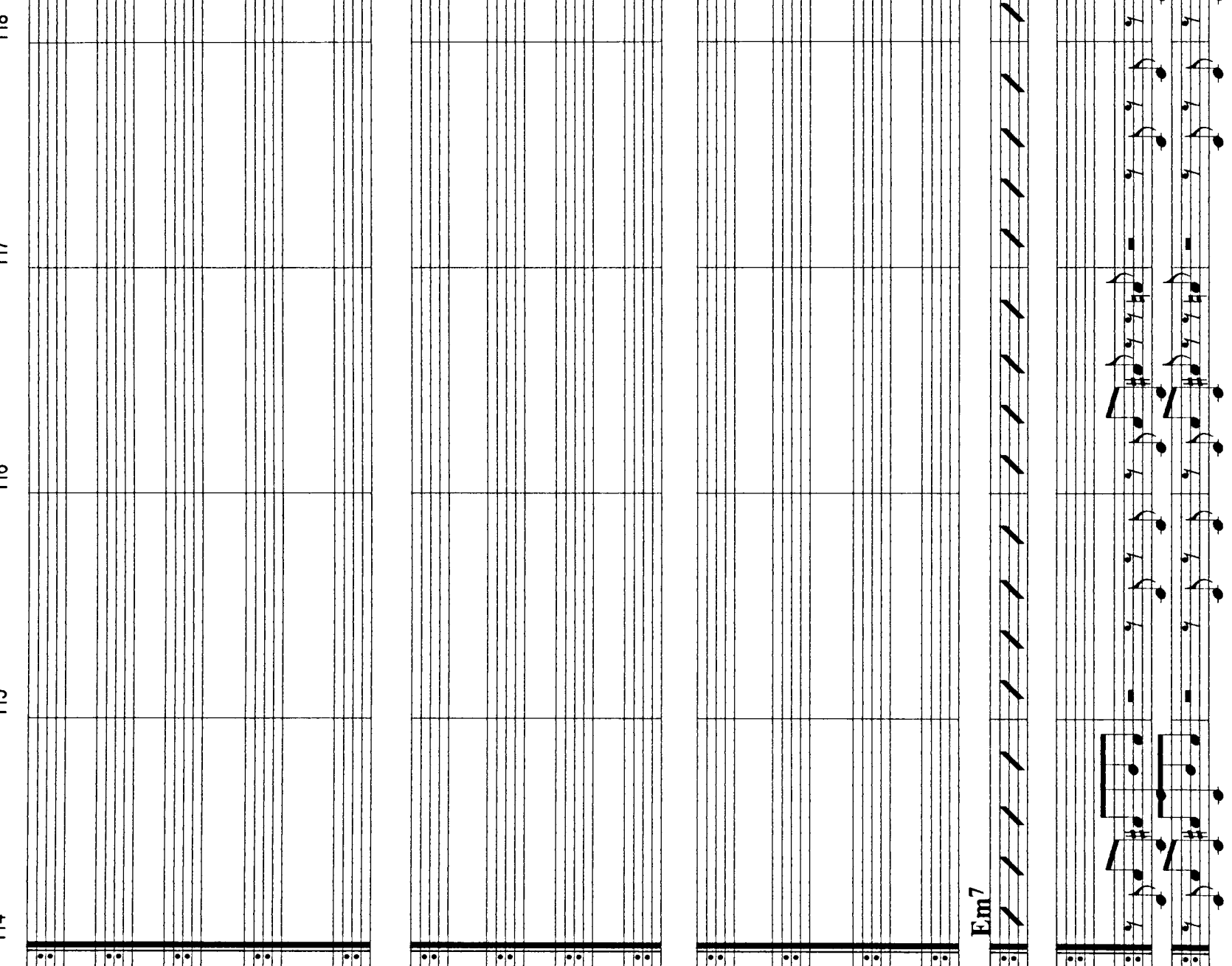

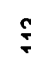
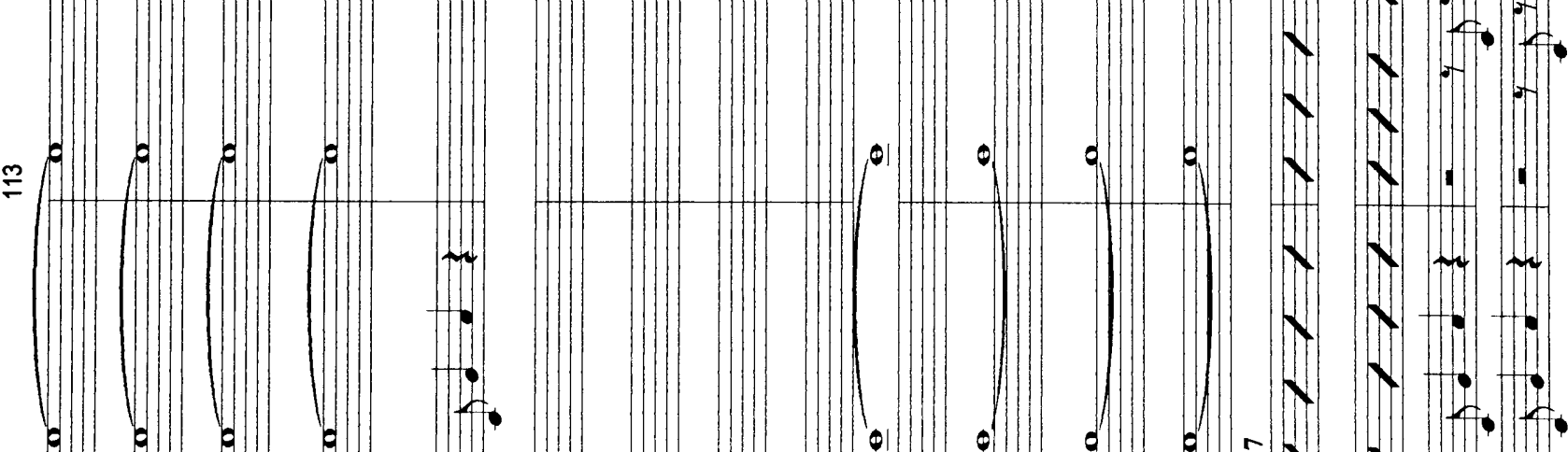

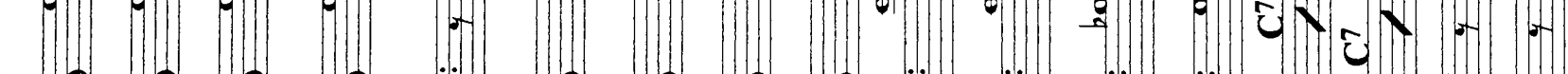

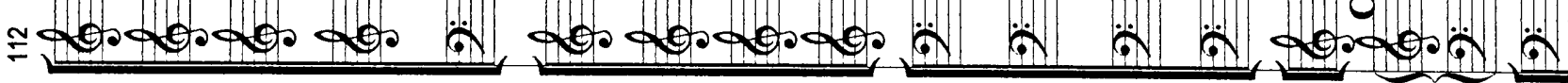




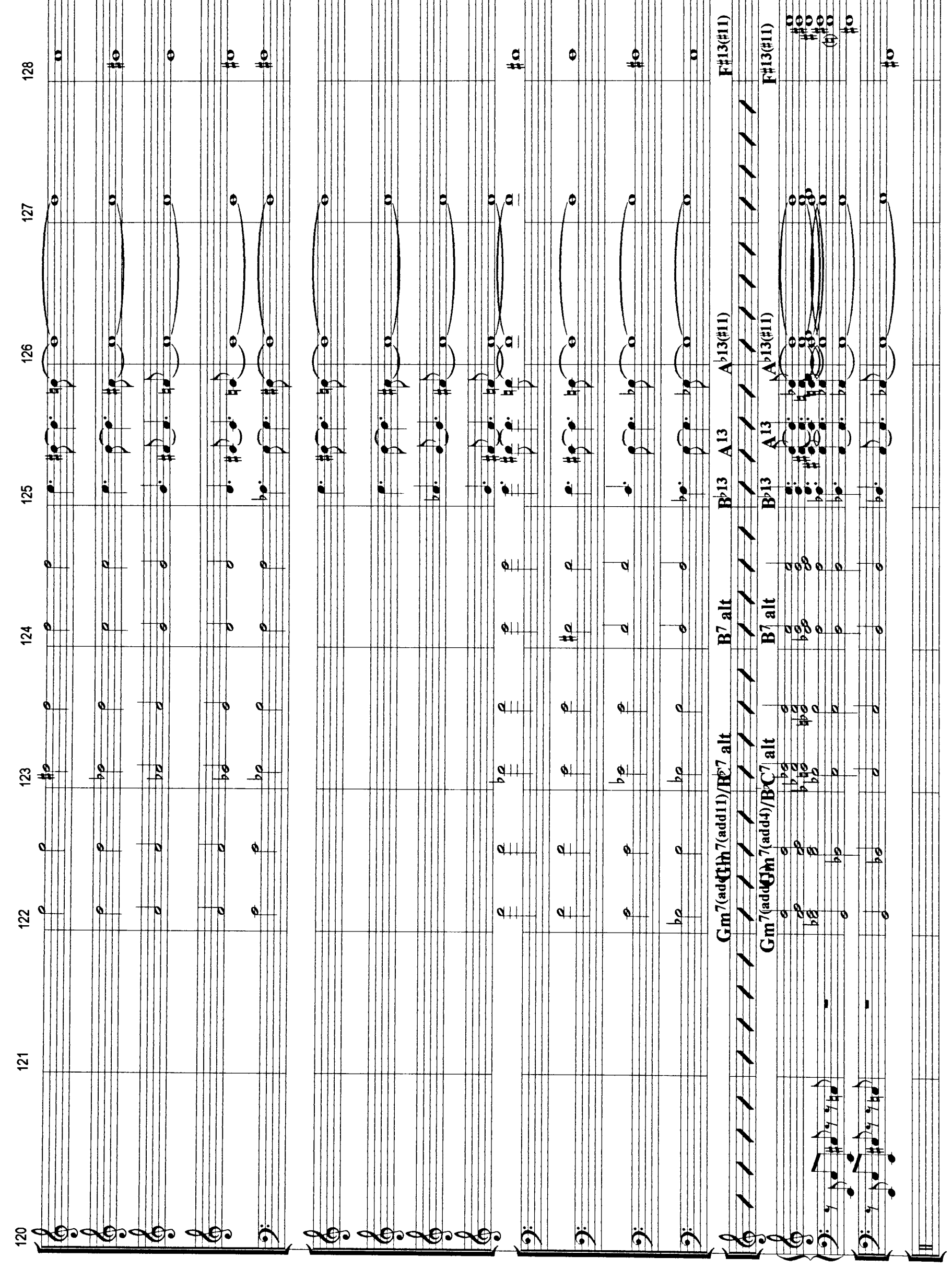


का मो का

$\because \div \div$

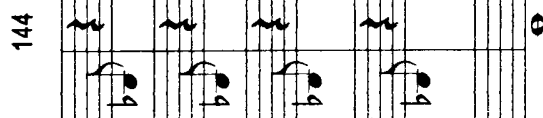

$i: i \cdot i: i !$

1: 1: : :

甲

tit

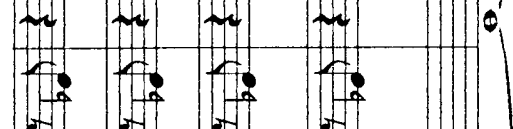

$\approx=$.

$\because: \div \div:-1:$

$\rightarrow \rightarrow-1 \rightarrow$

三: \#: \#: : : : :

$\rightarrow \cdots \cdots$

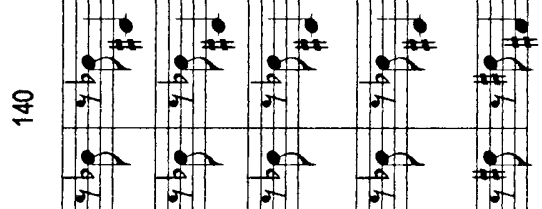

1 H

\begin{tabular}{llll} 
& & & \\
\hline
\end{tabular}
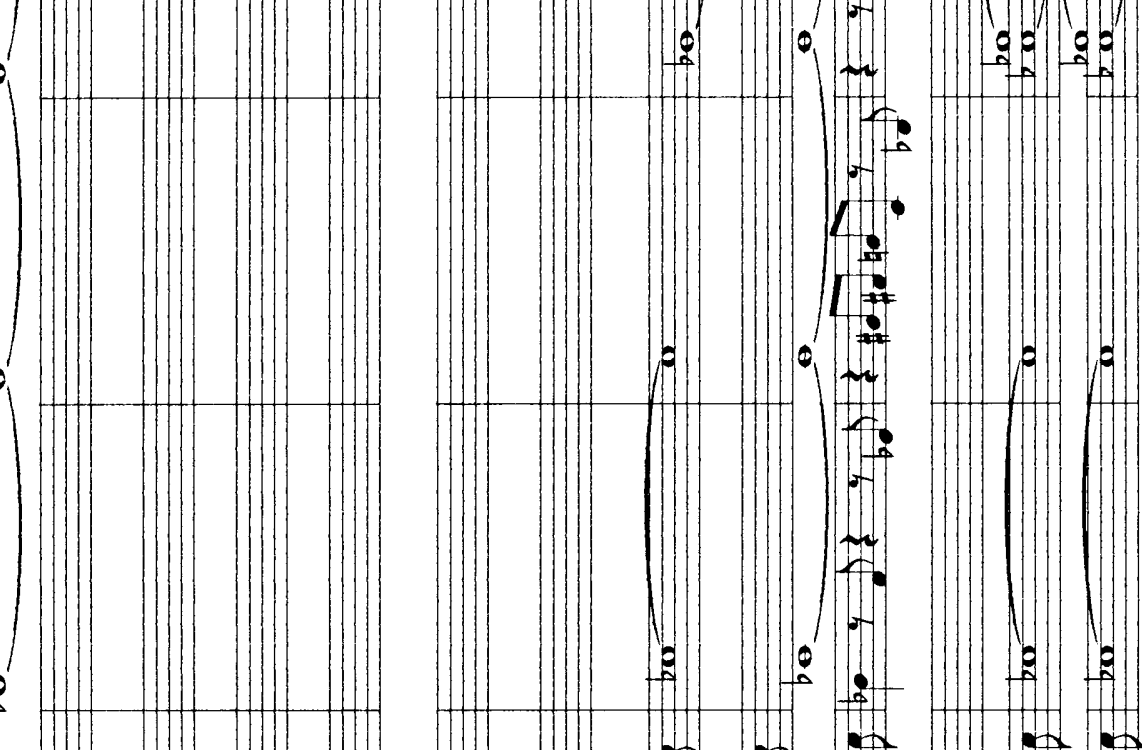

(n)

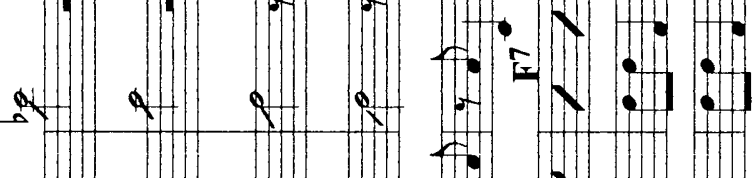

$r^{\circ}, 1: 1 \cdot 1 \%$

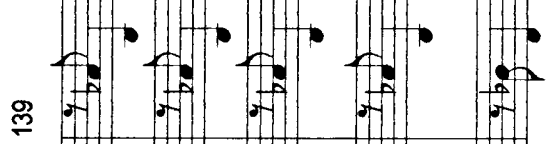

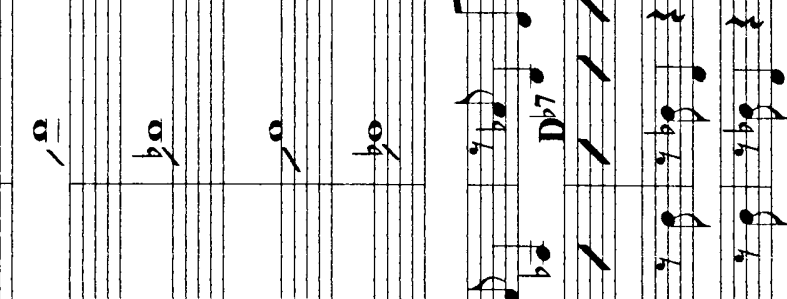

吅

- . :

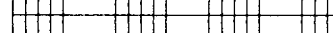




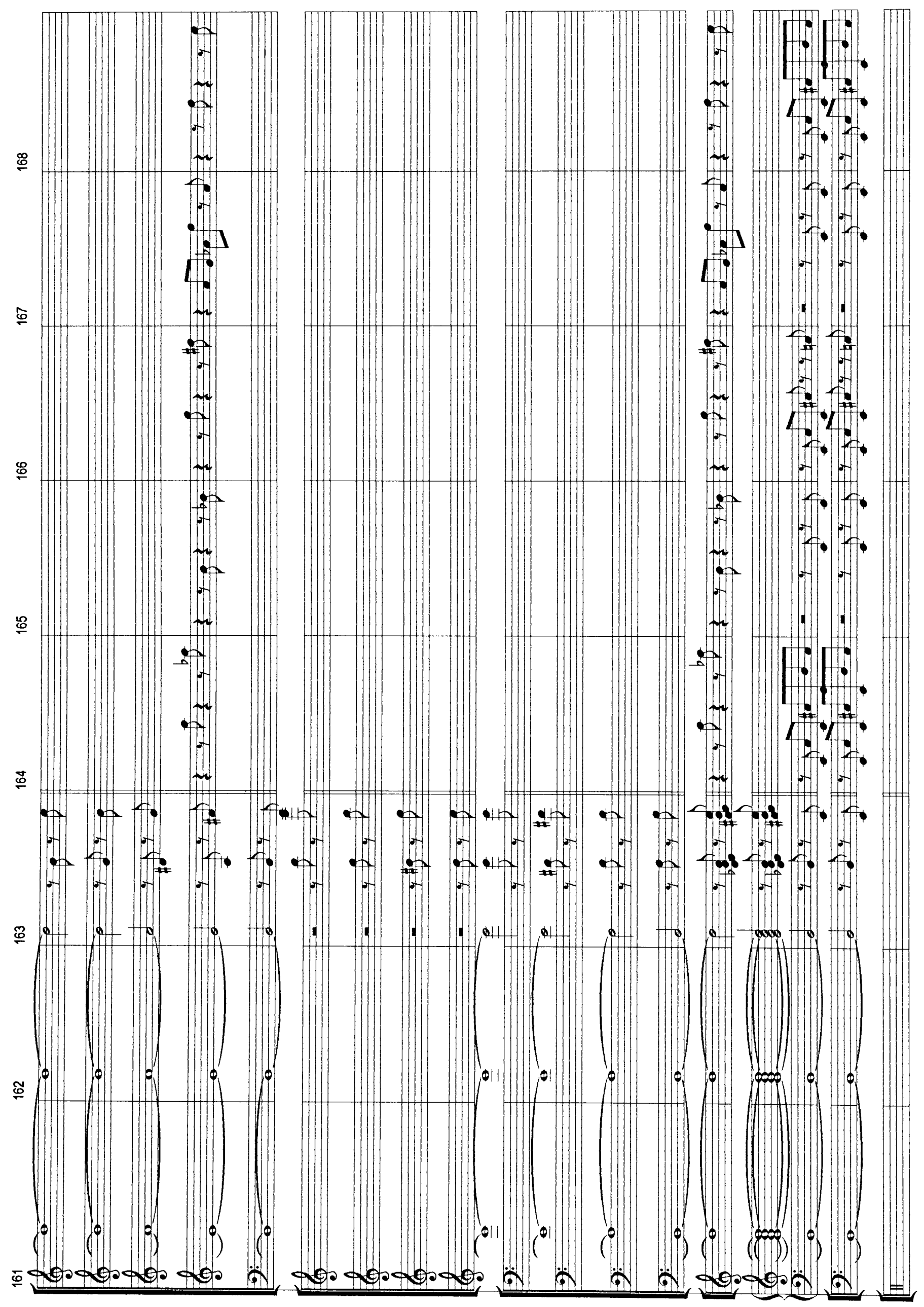




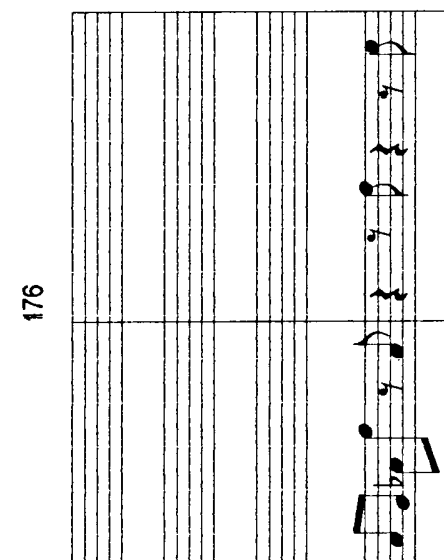

$\stackrel{2}{2}$

$\Xi$
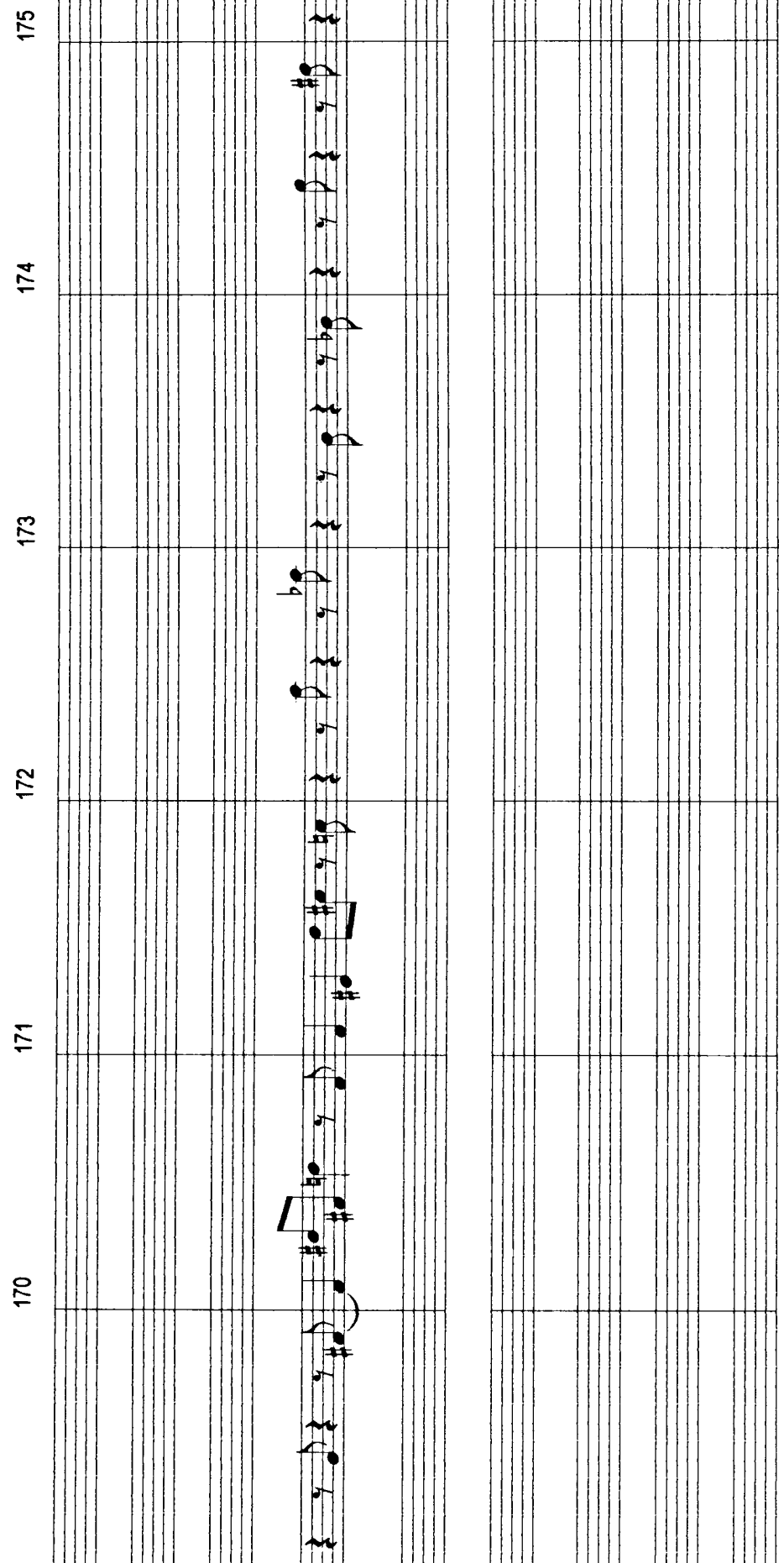

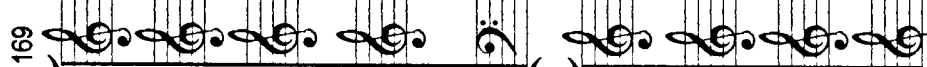

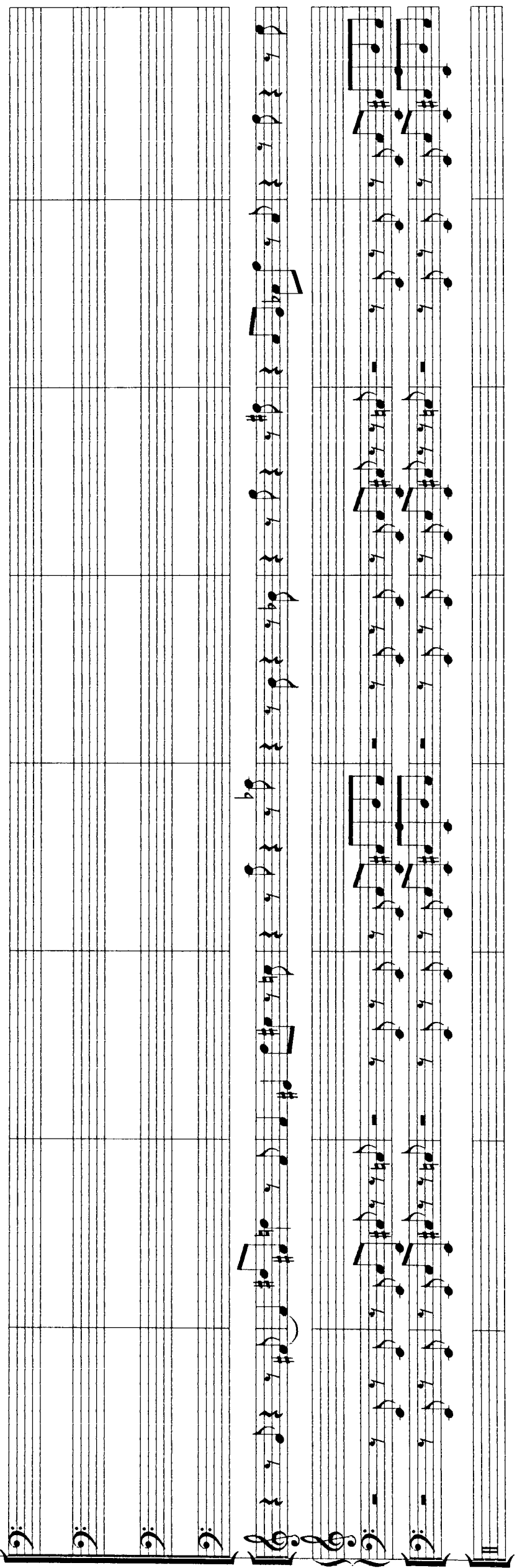




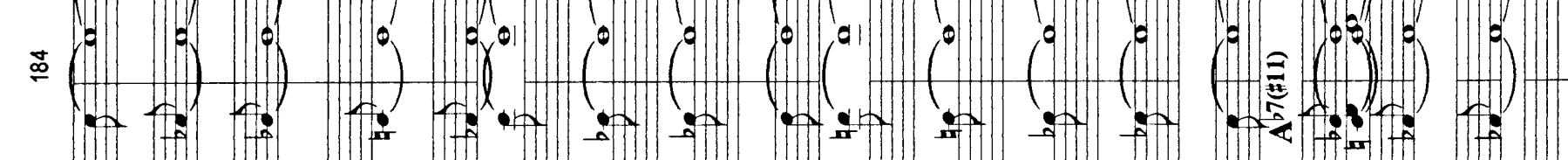

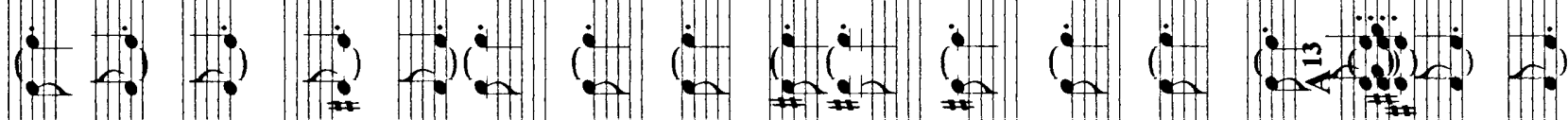

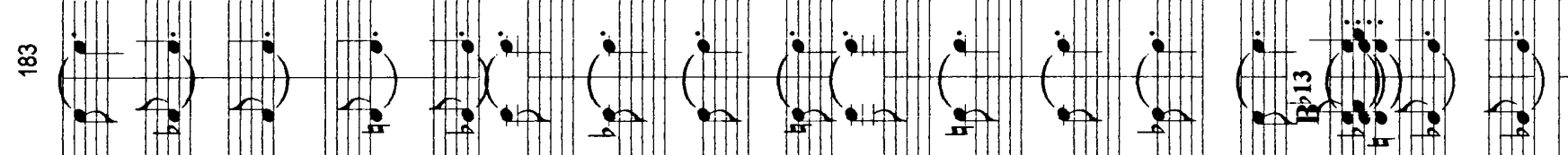

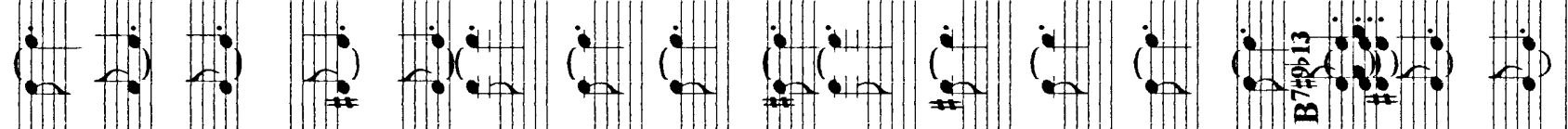

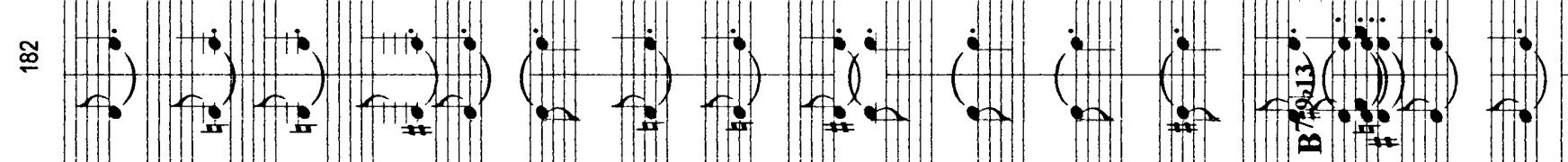

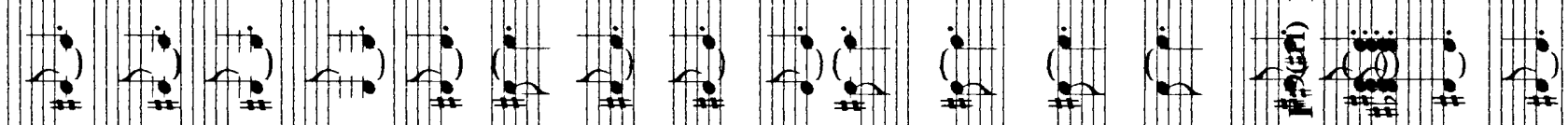

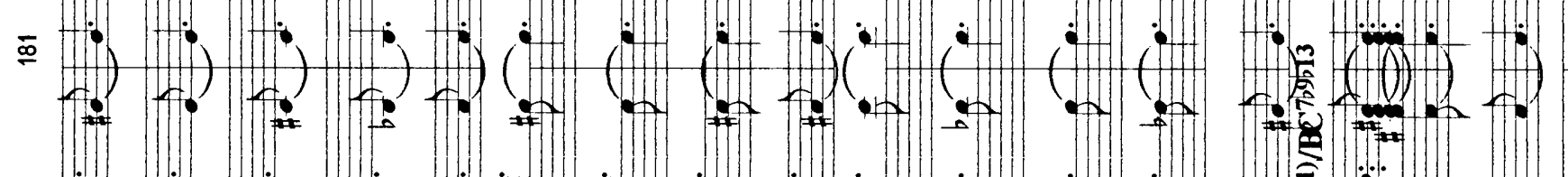

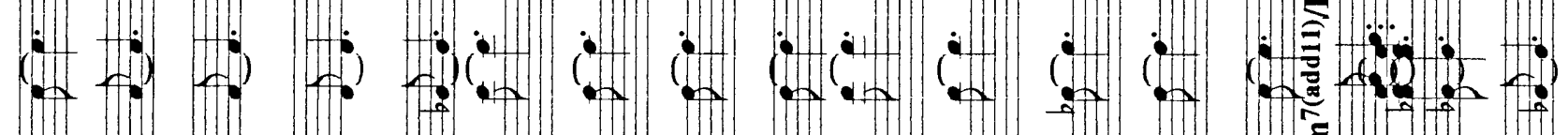

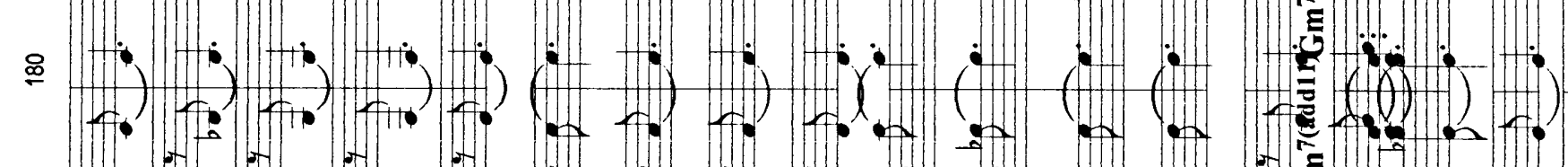

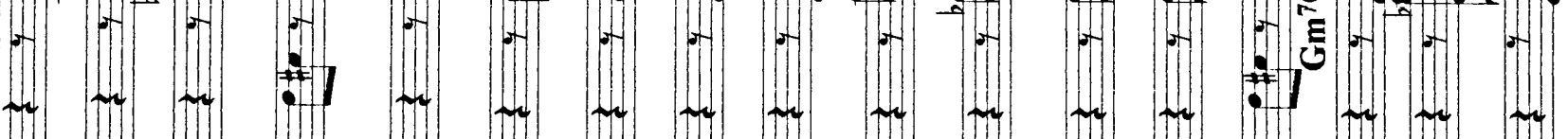

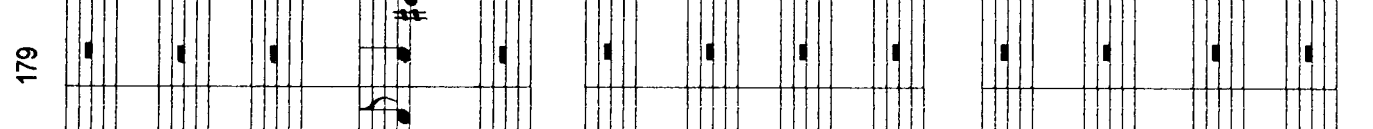

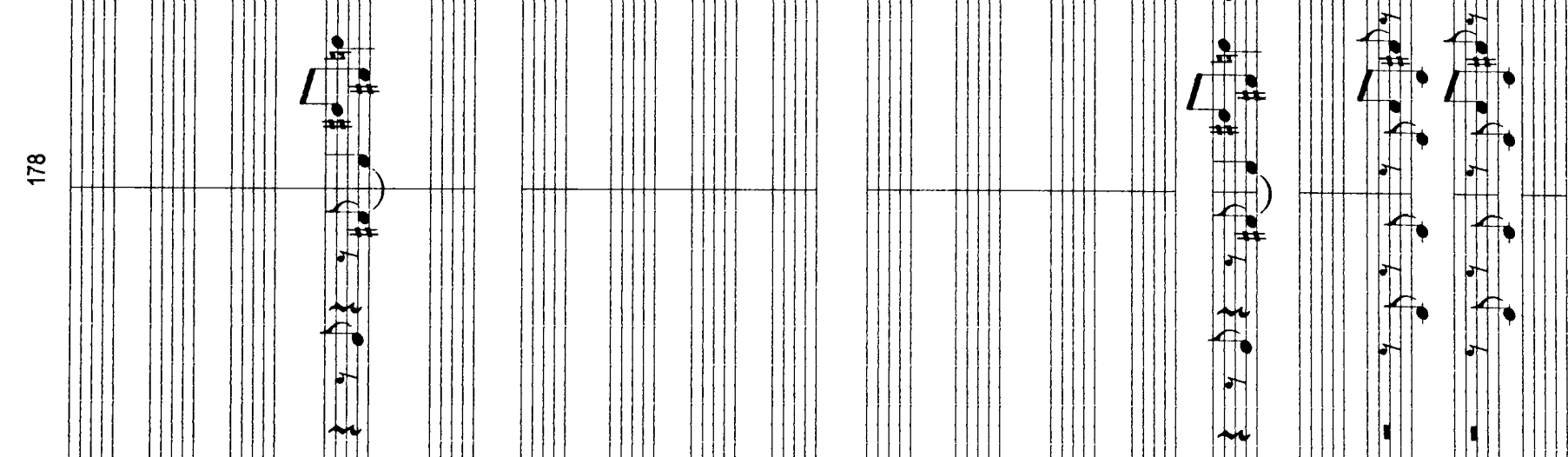

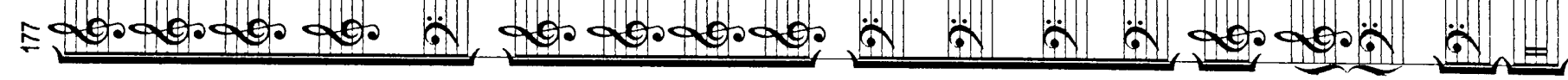




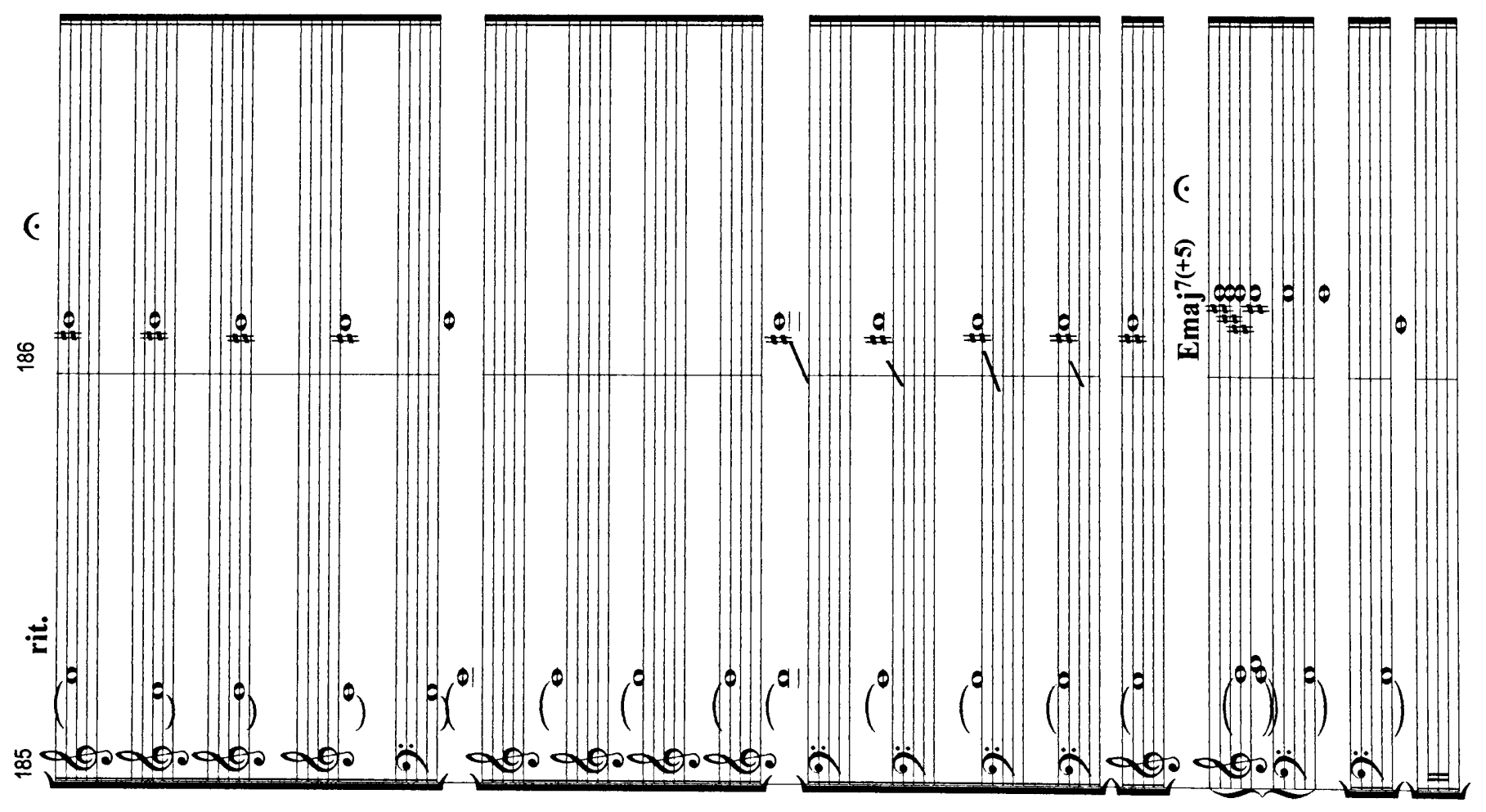


यद्य

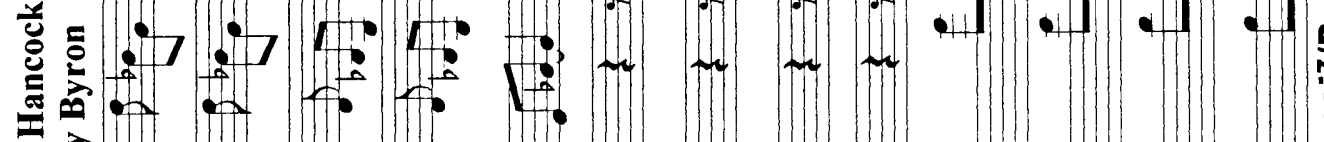

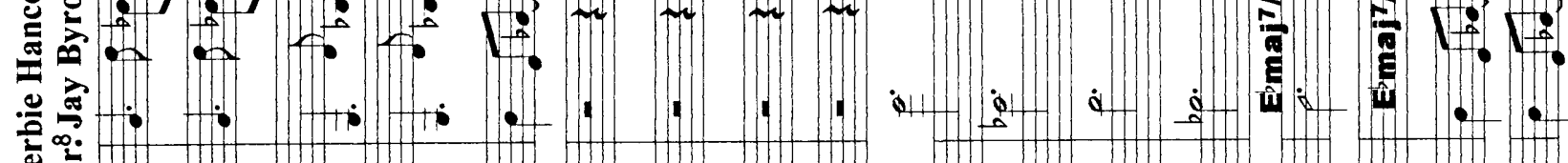

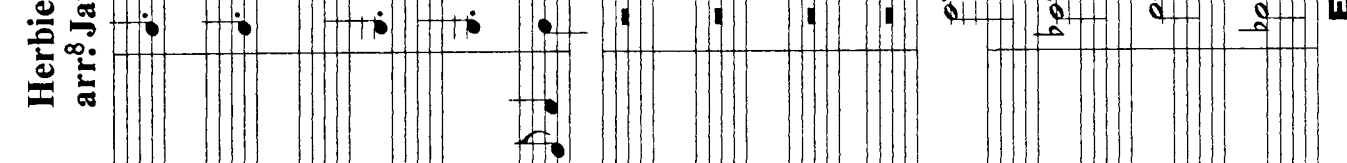

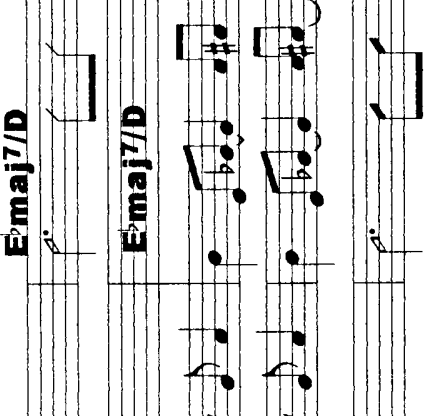

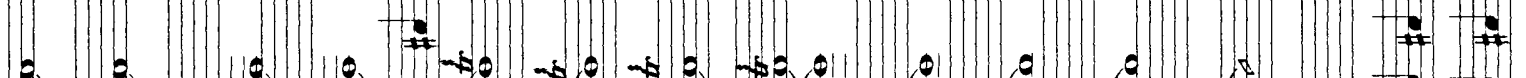
(1)

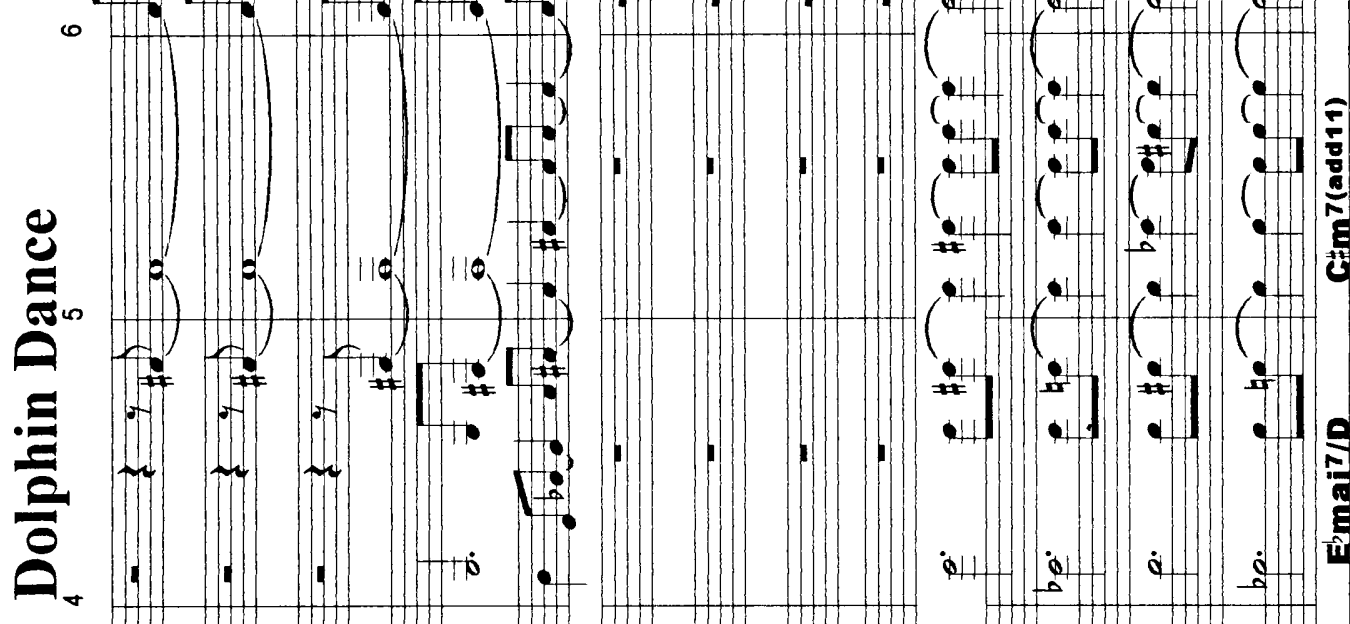
(f) n: $: C$

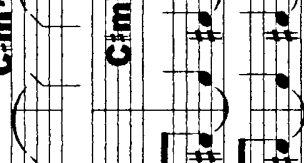
is E (:

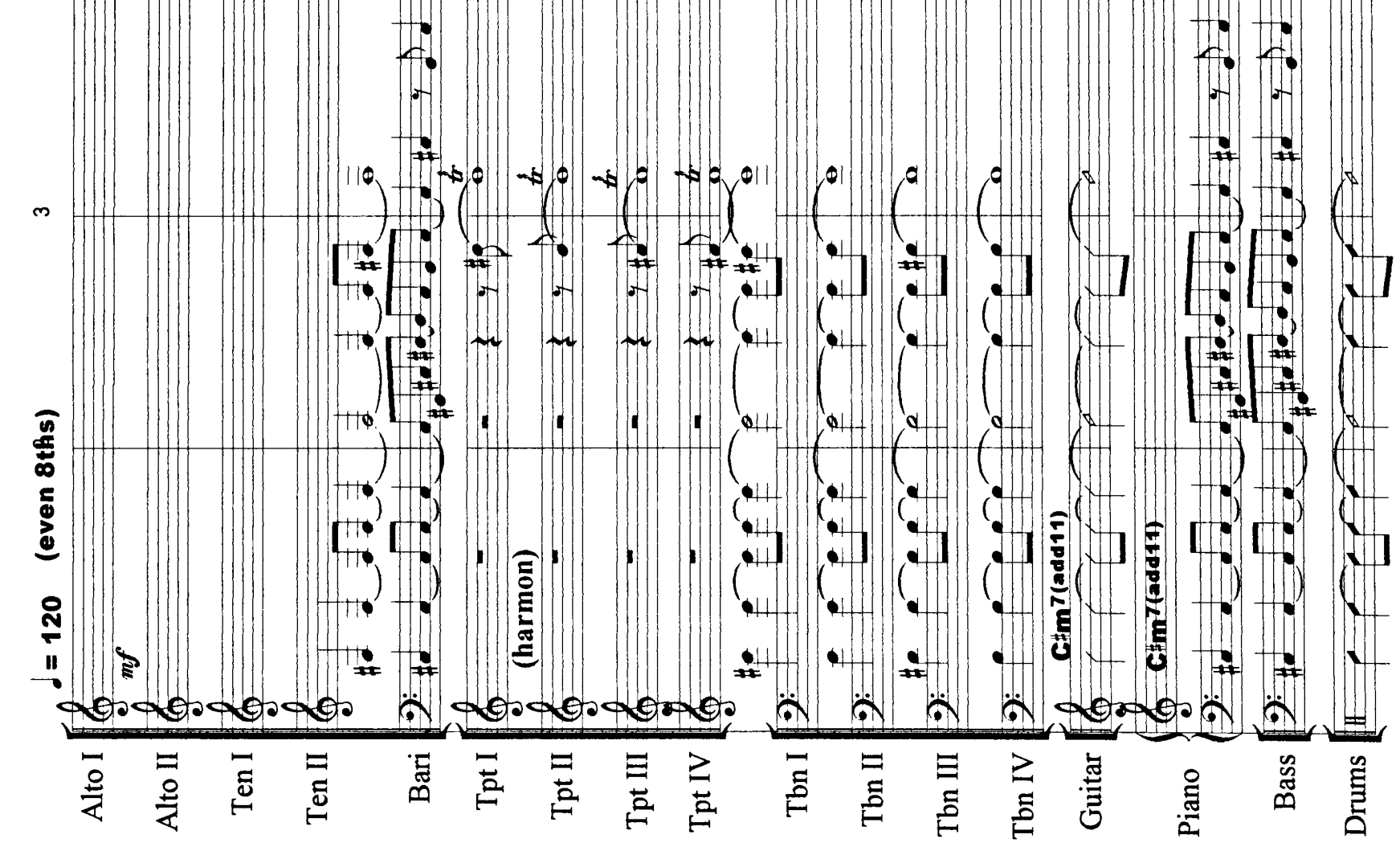


$\because: \because \because: \because$

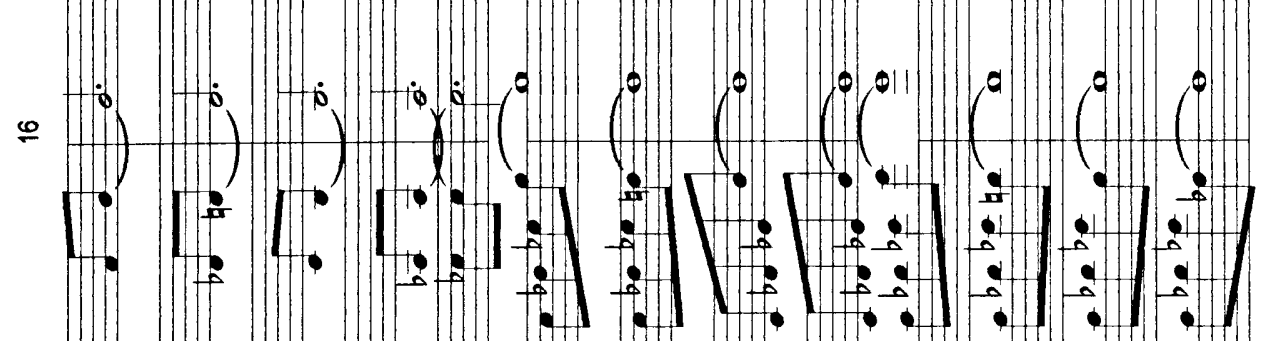

$=\left(;: 1: 1: x^{3}\right.$

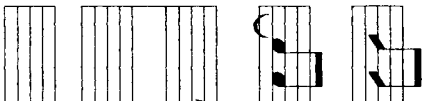

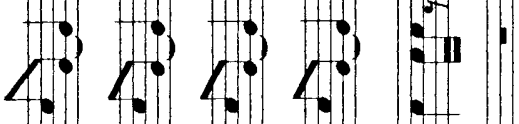

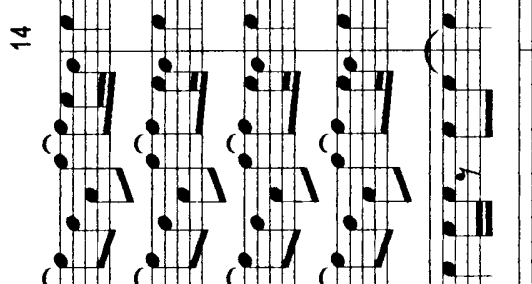

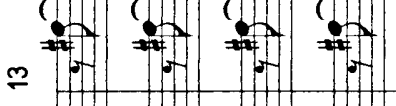

:

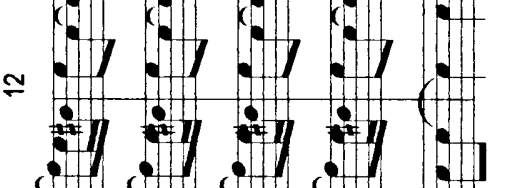

$+\because: \div:$

$=$ Non

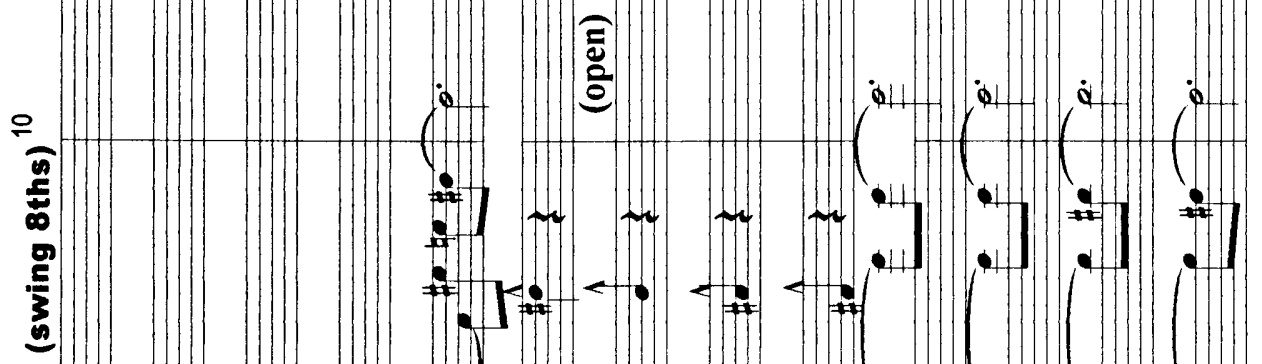

4 in in in : : $1: 15$

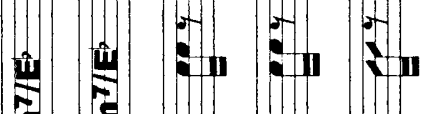

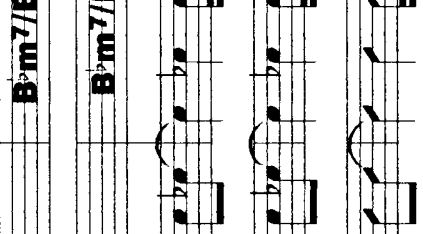

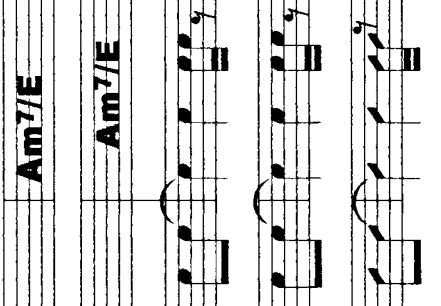
는 (1) :

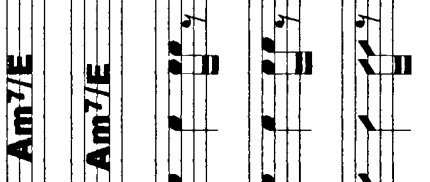
: $1: 1$ H. Bn in : i : i I. || || ||| - + - -

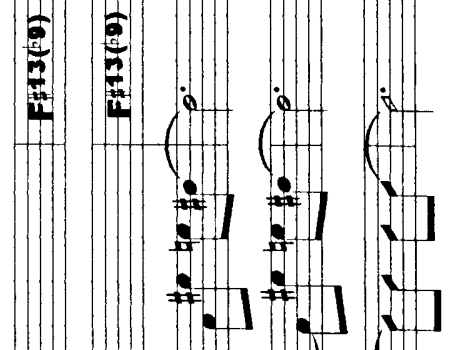

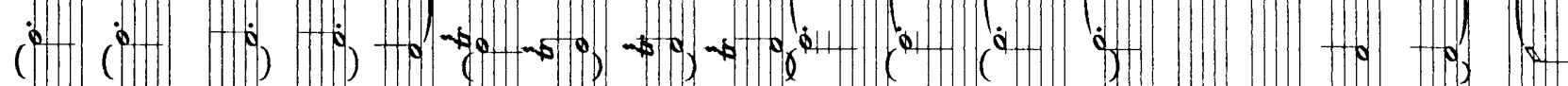

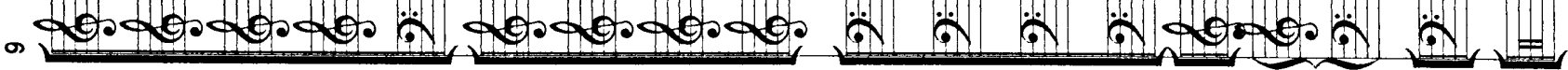




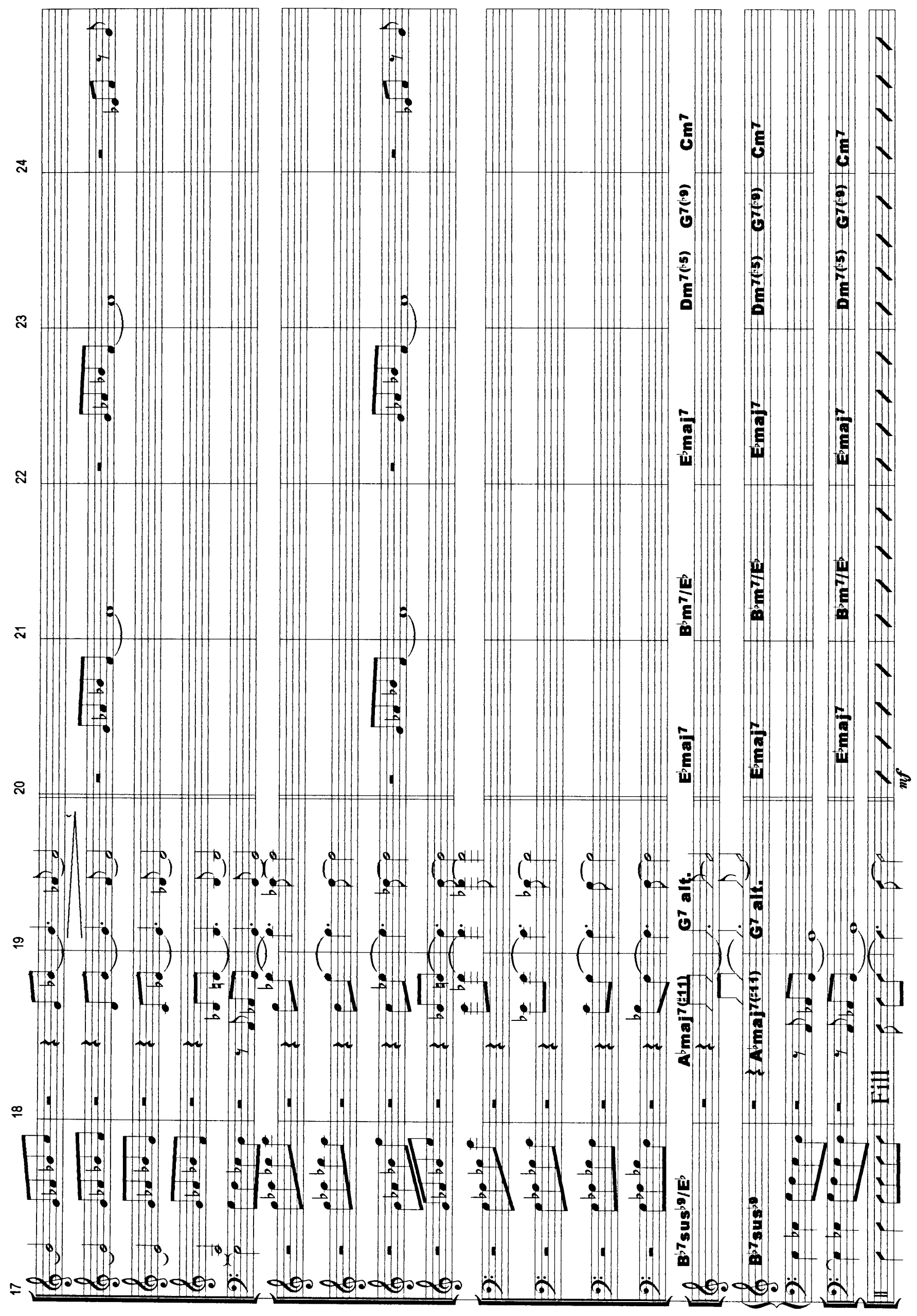




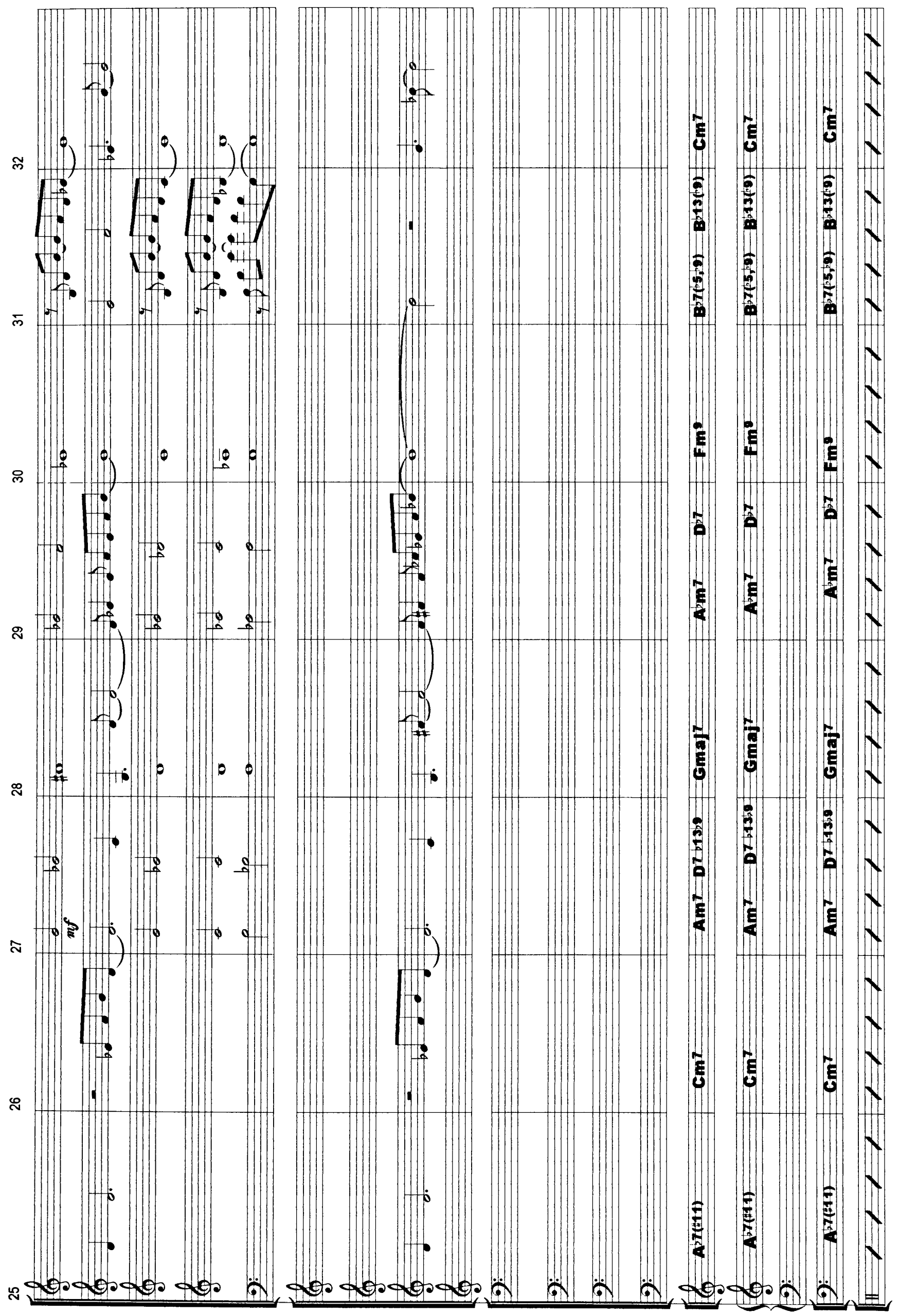




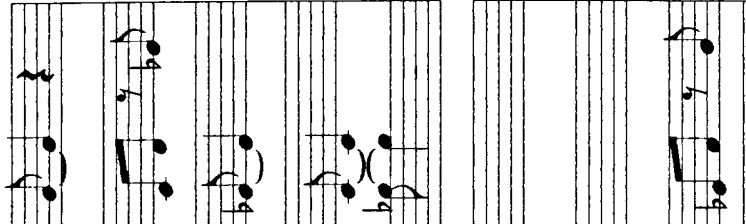

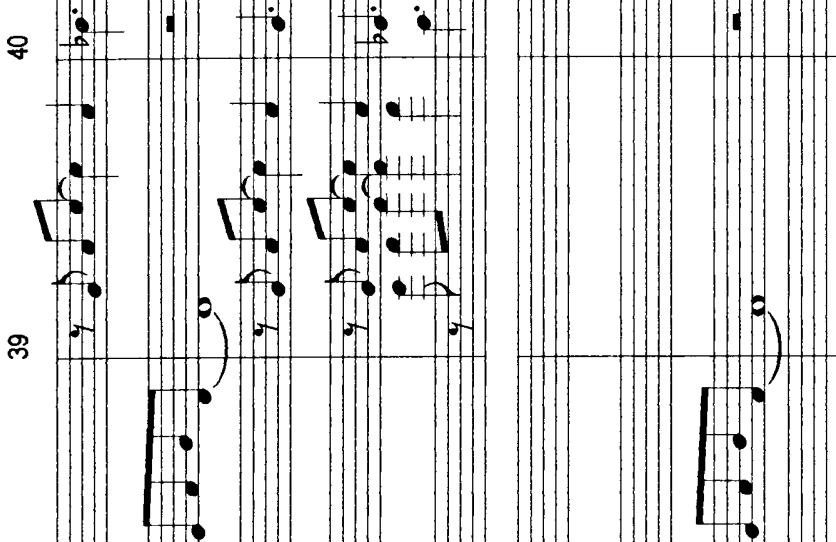

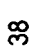

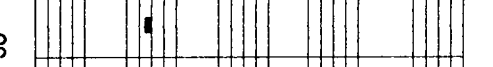

.
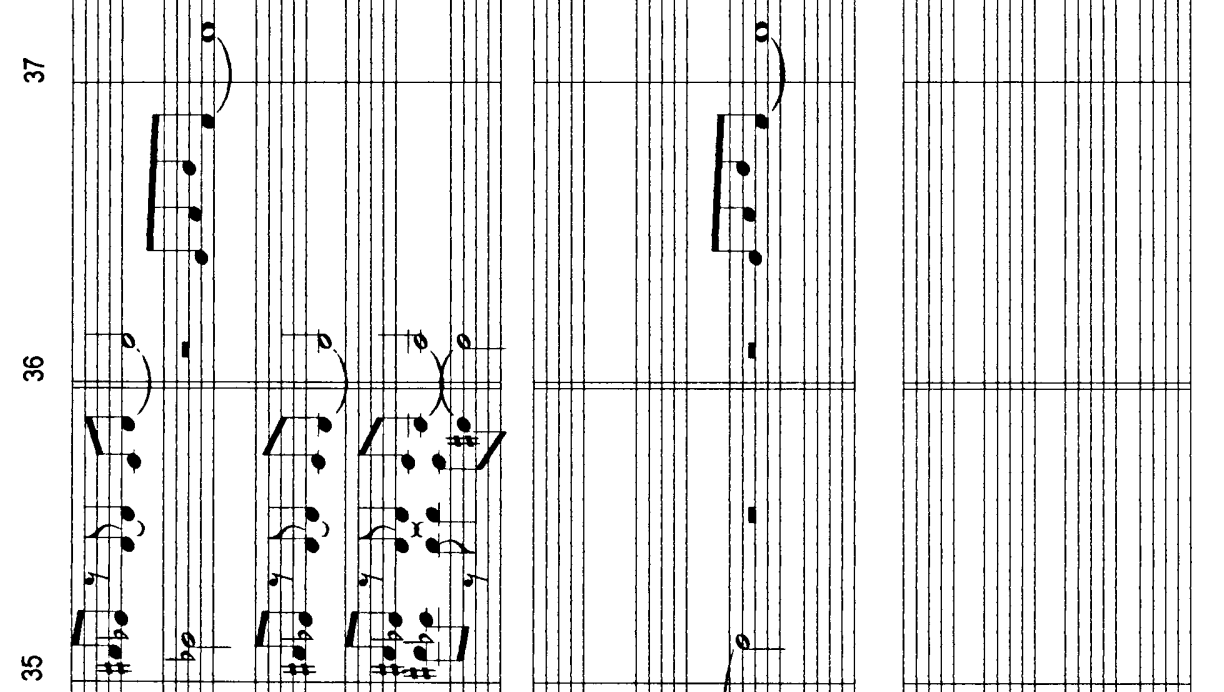

-

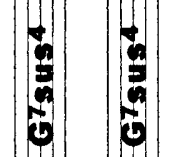

:

:

1. . (1.
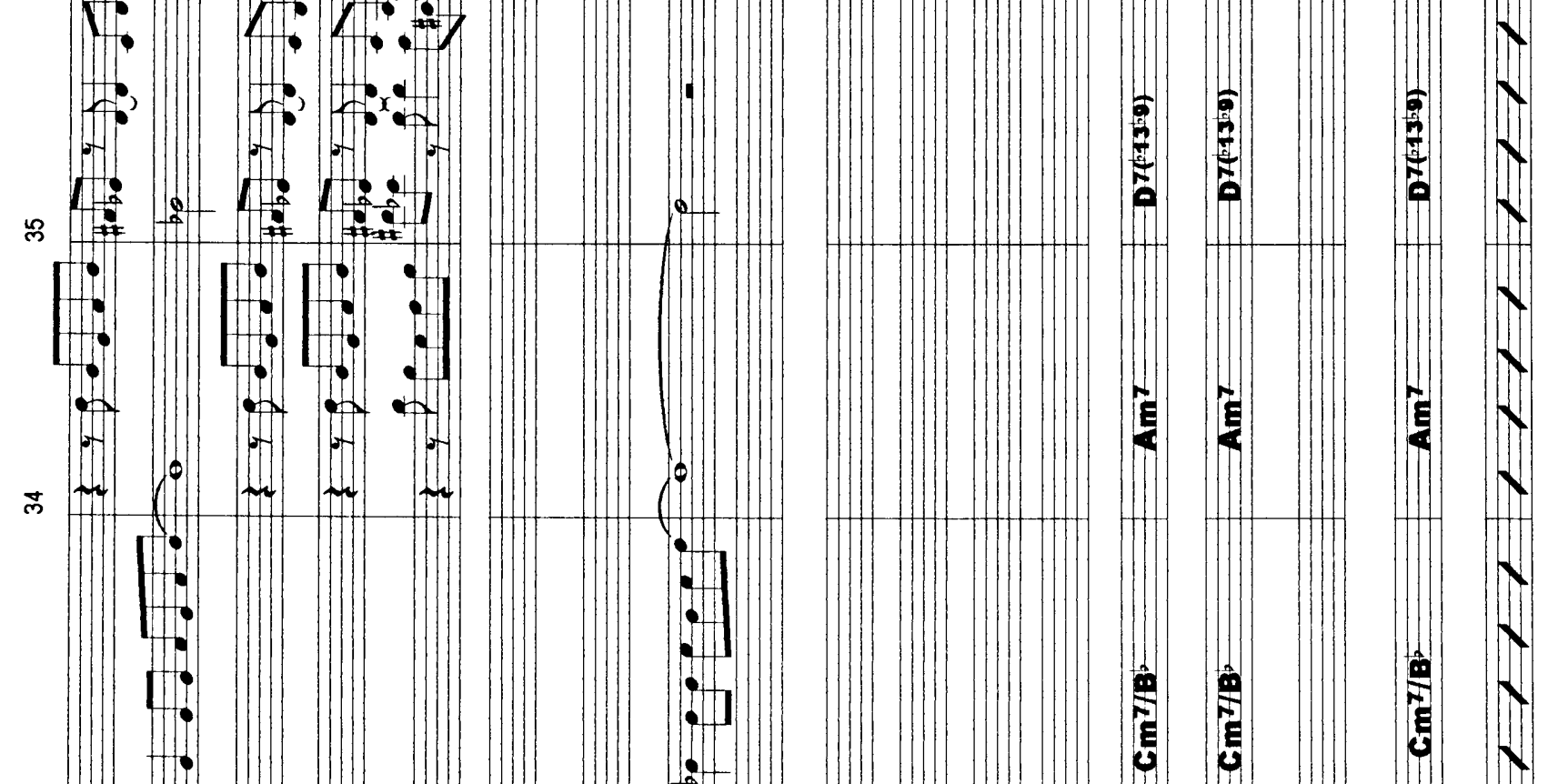

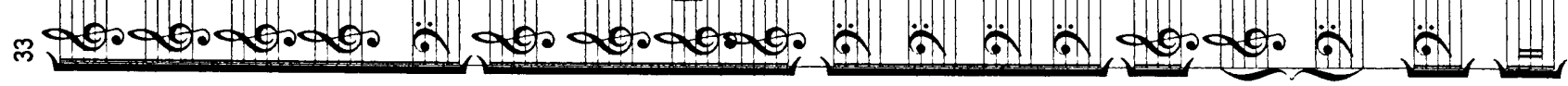




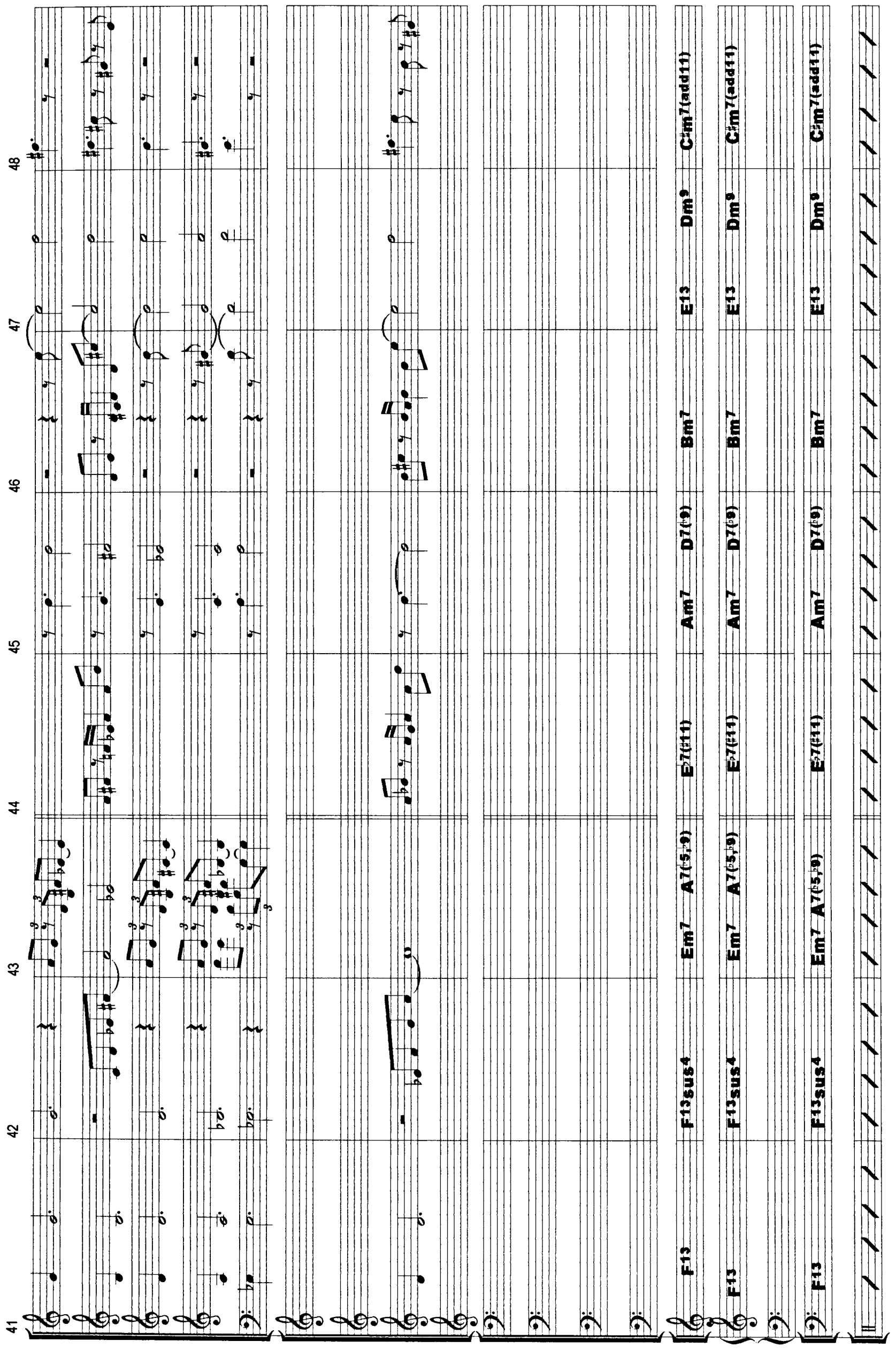




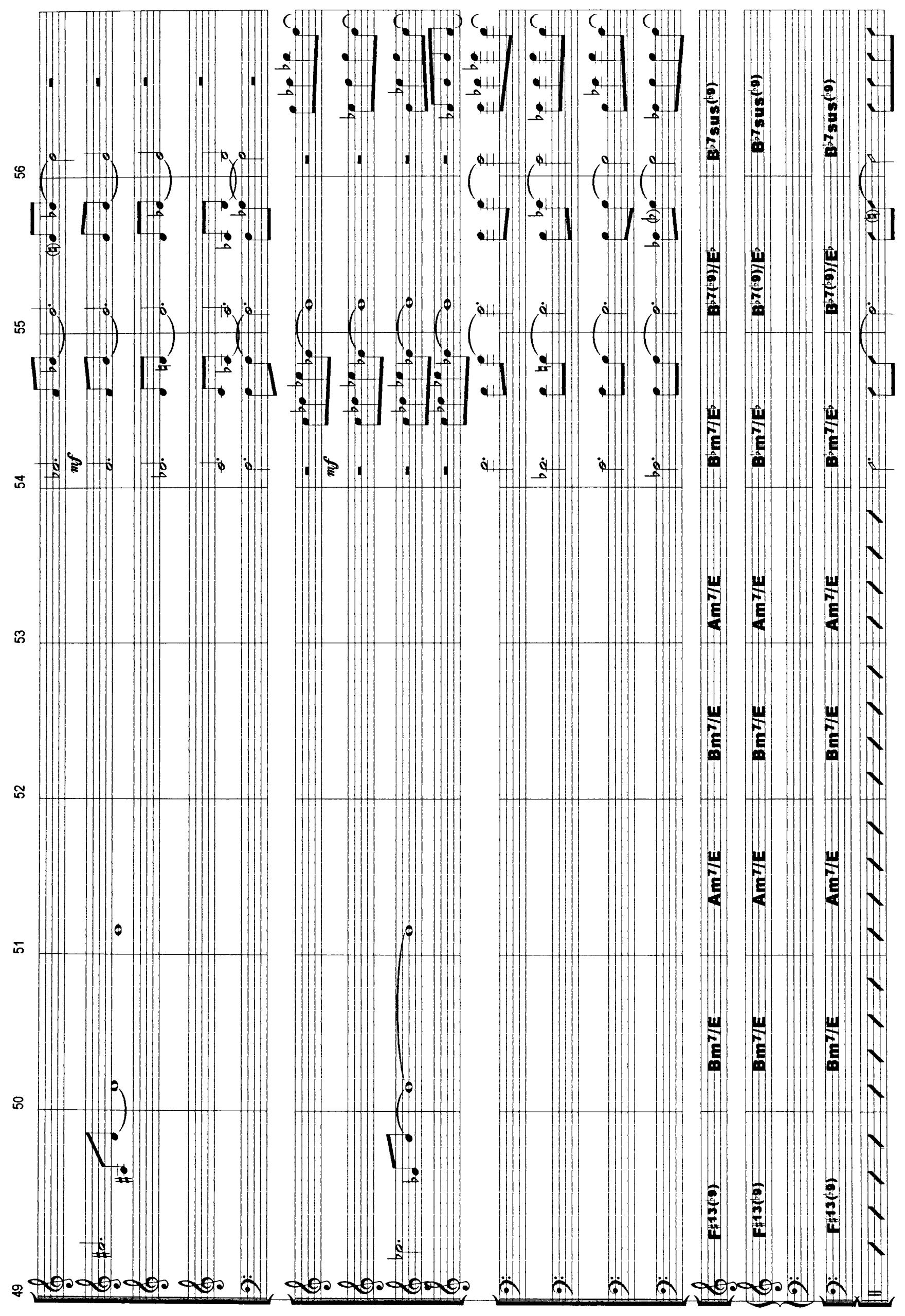




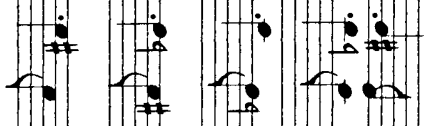

F:P:B:

Б)

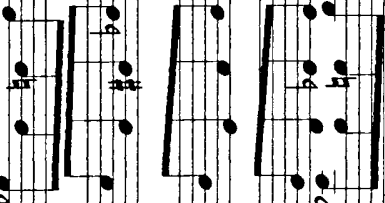

:17: 1:, 1::

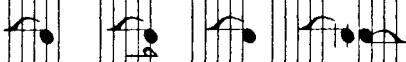

8

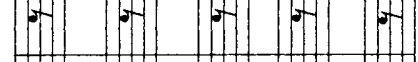

m n w w w

1140

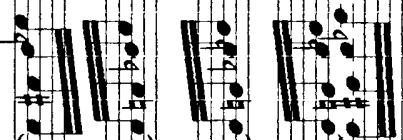

(i) +4 मक

品

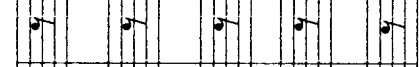

影玹

5

:

s

$\checkmark$

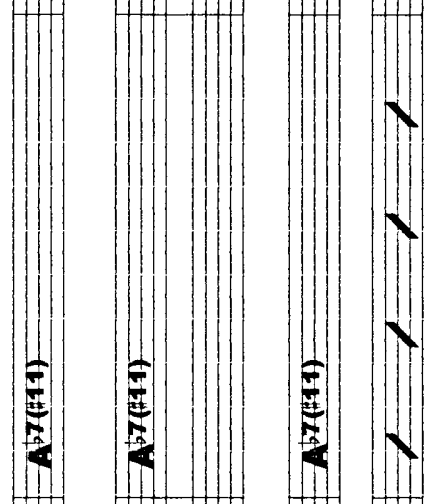

$+$

N

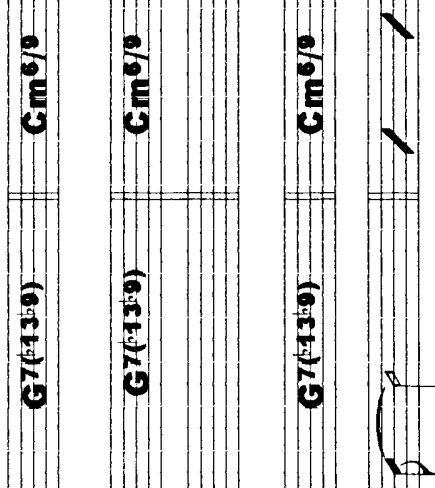

:

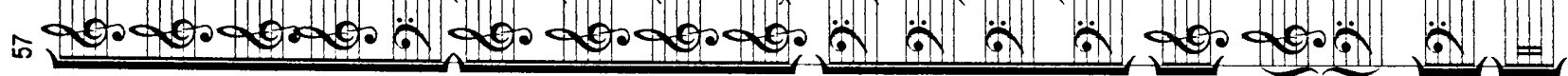




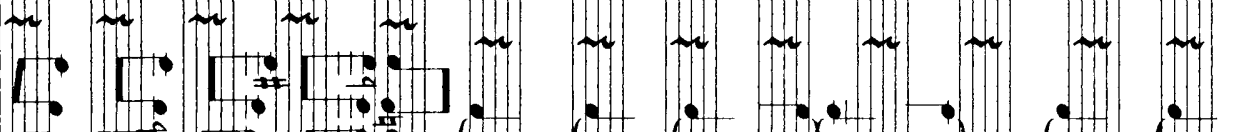

8

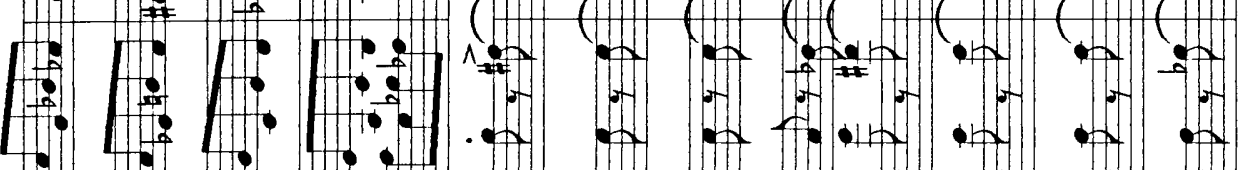

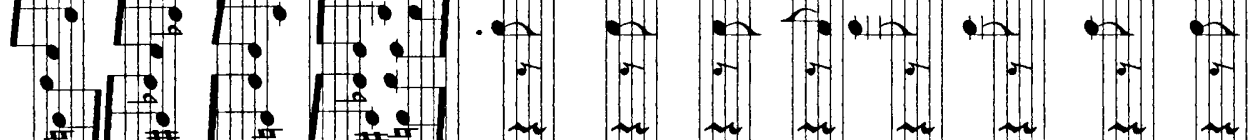

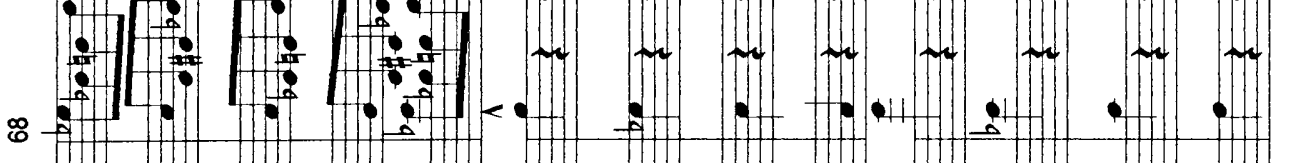

.

.

然

- I:

$\frac{\lim ^{\infty} m^{\infty}}{m^{\infty}}$

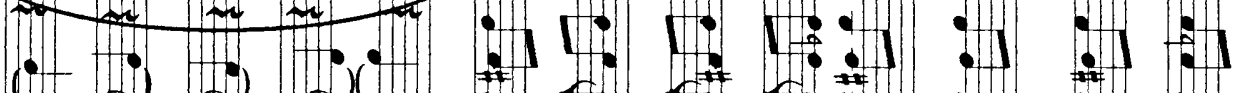

ह

$\sqrt{1}$

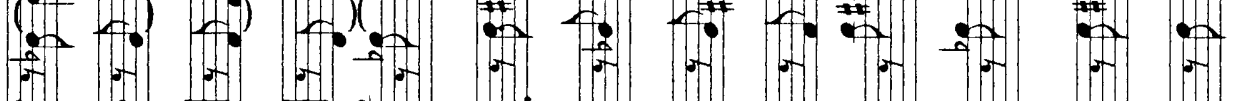

$8 \cdot 1: 14: 40$

s

1.1

.

音

un

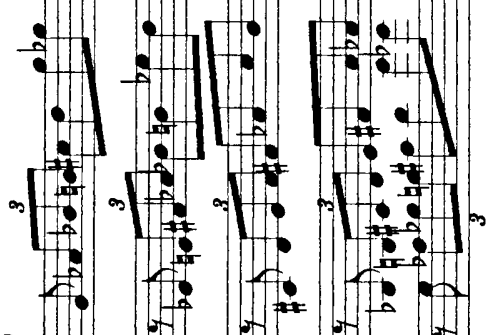

๕

(n)

घ : $:$ : $: \dot{2}$

高

:

N

1

$\sqrt{2}$

$\underbrace{}_{0}$

6.

E E

-1.

E

Th

E

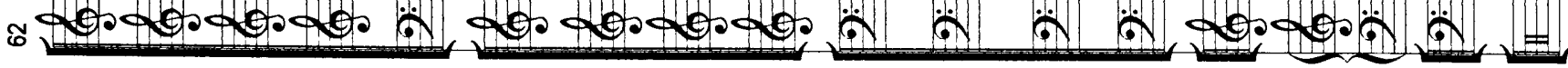




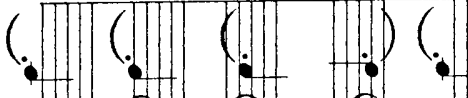

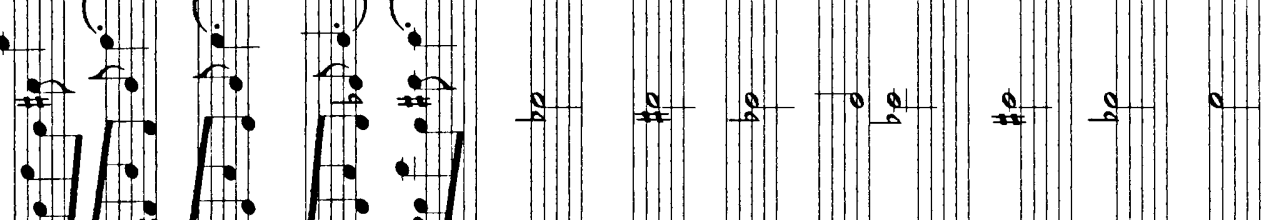

$=1:=0 ;: \div$

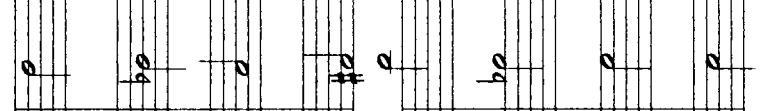

:

$\because 1: \div \div$

D

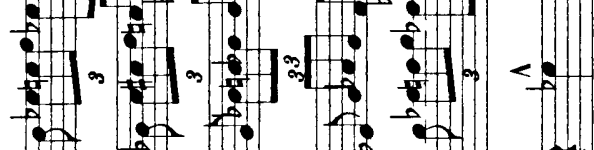

$=2: ?:=$

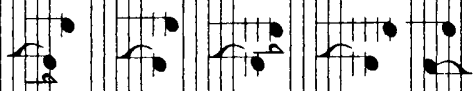

Fi: $:$ : $: 3$

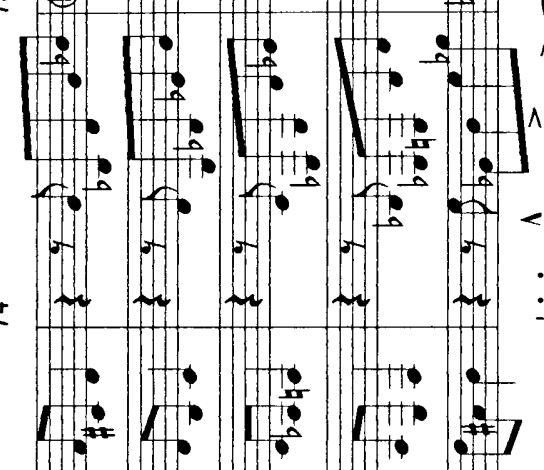

(:1) (c)

$=$

F:F

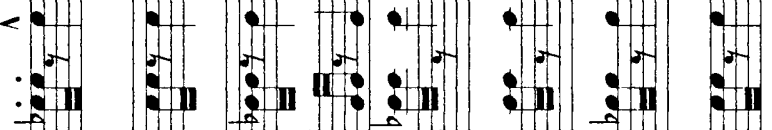

:

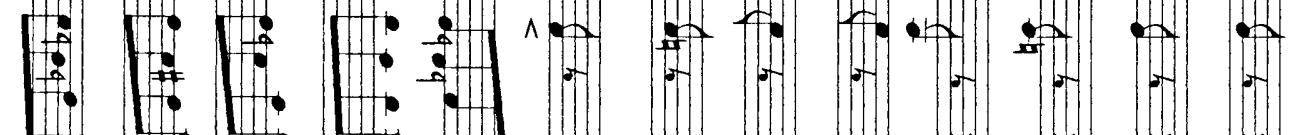

: : : $\ldots \ldots$

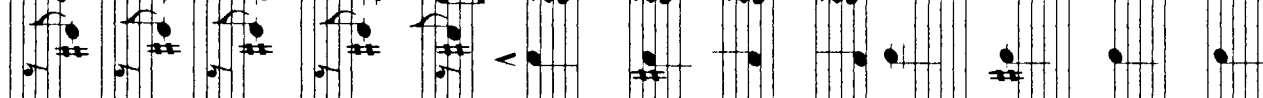

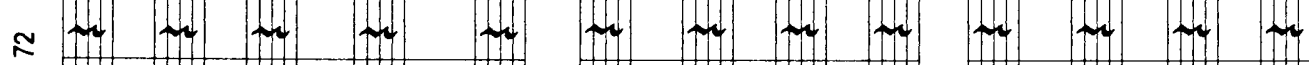

:

T: $: \pi: \pi$

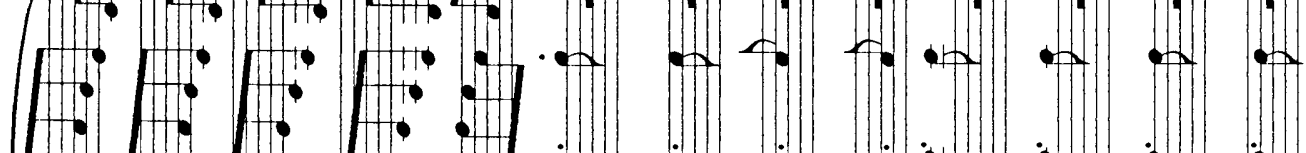
: : : : : : $:$

- ․

:

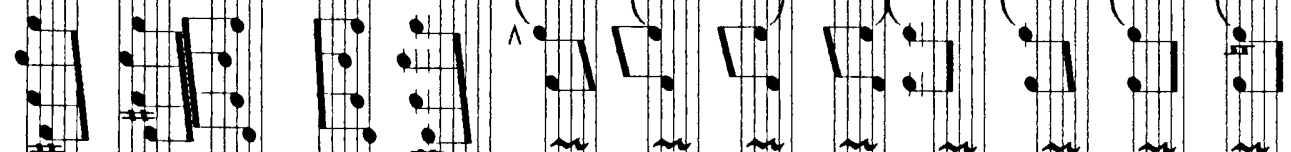

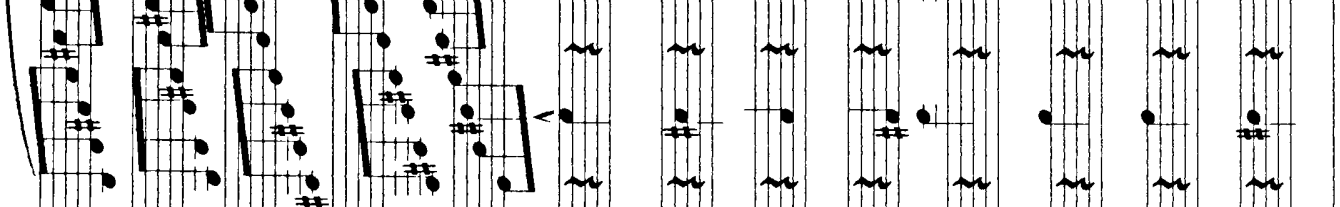

部

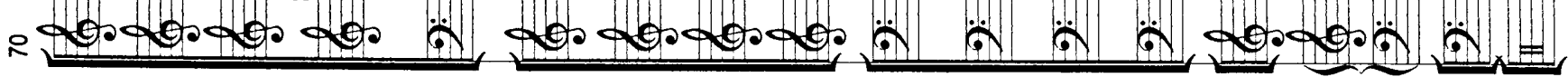




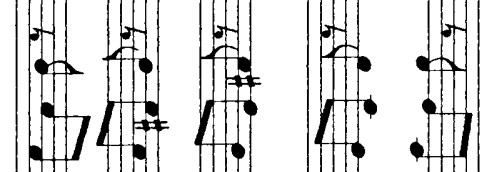

$\therefore: 11: \div$

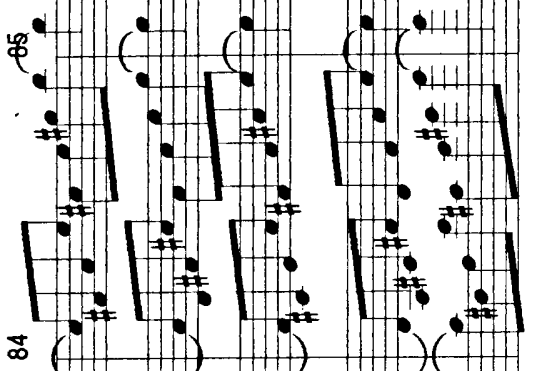

$\left.\lim _{a}^{\infty}\right|_{a} ^{\infty}$

$\because 11: 0$

๓

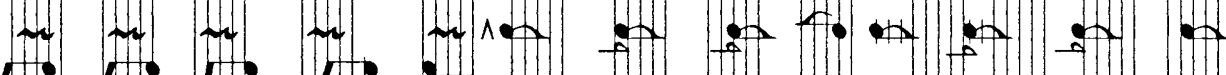

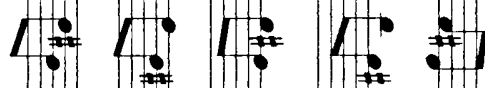

$: 11: 1: \vdots$

$: 1: 1: 1:: 1$

$: 1 \div 1 \div: \div$

क मी को

$: 1: 1: \vdots \vdots \vdots 11$

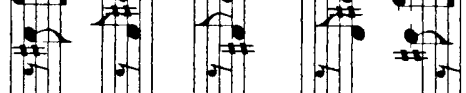

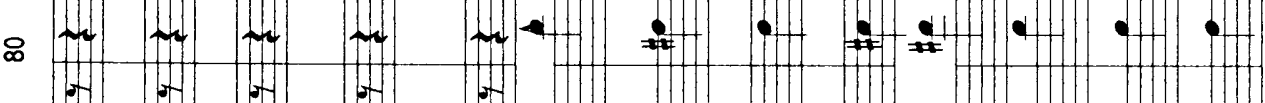

$\rightarrow \cdots$

$\because 1 \div \div$

$\because 1: 1: 1: \because:{ }^{\prime}$

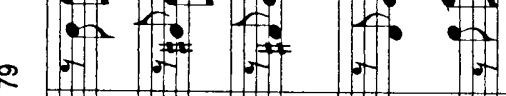

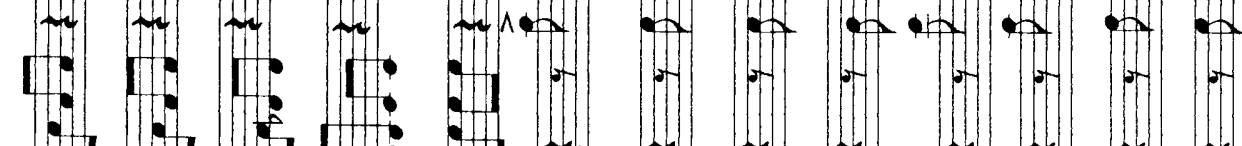

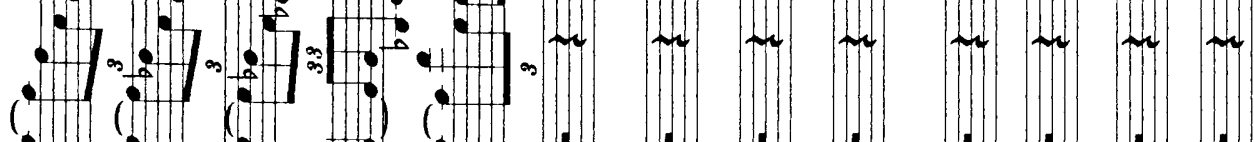

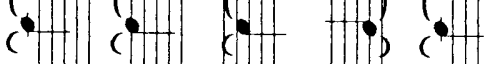

soroonos a

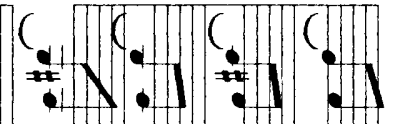

(1)

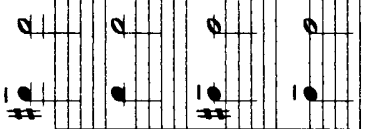

พ⿳亠丷⿵冂丶

$+1$

要

(1)

$\underset{\boldsymbol{u}}{\boldsymbol{\mu}}$

․․

$\sqrt{ }$

急

(2)

E

竞

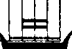




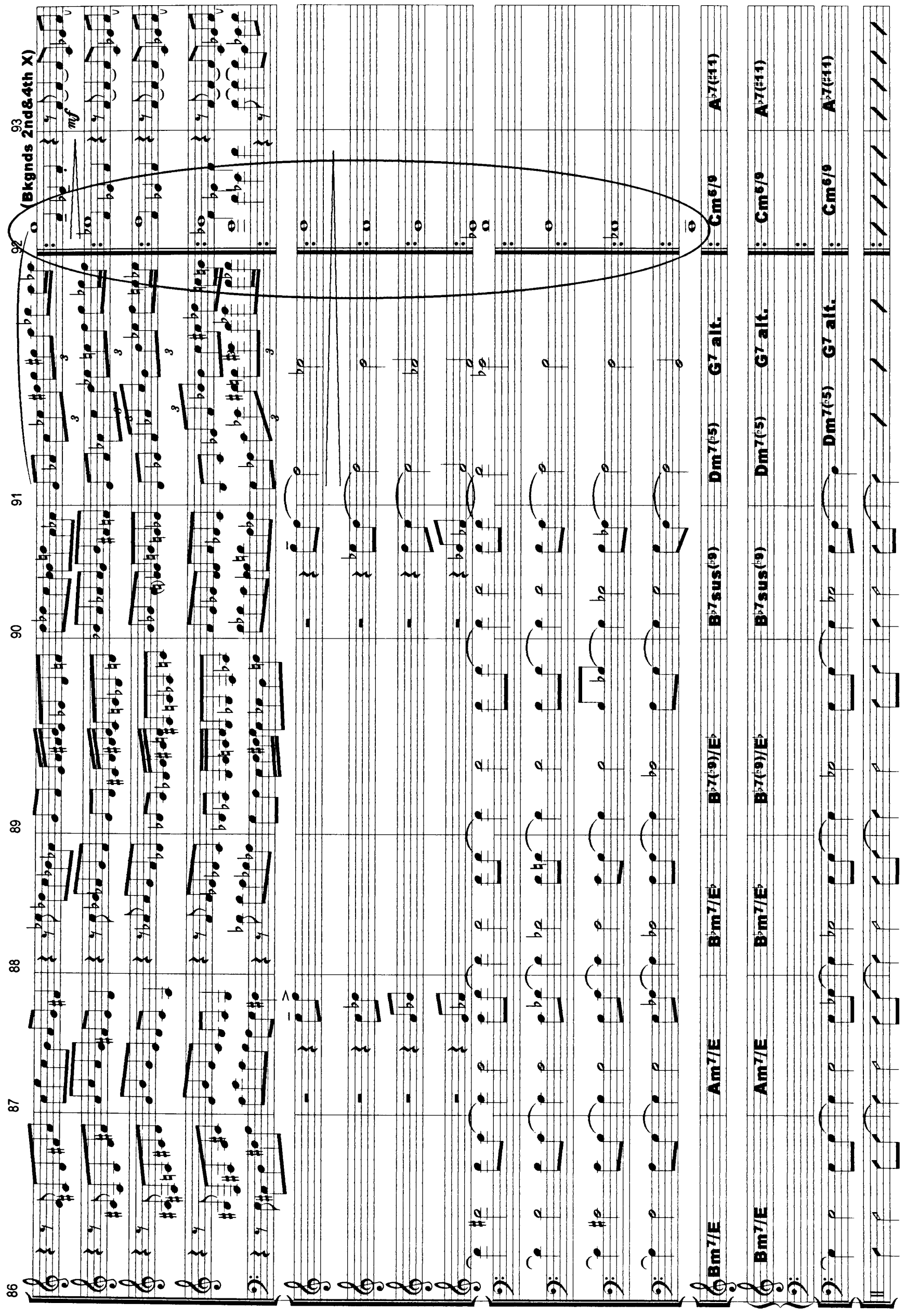




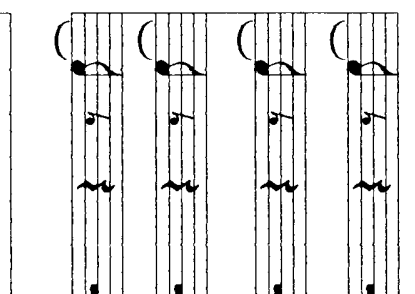

(1)

$\sqrt{3}$

$\frac{8}{\|} \|$

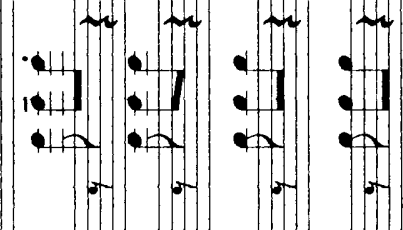

+11 :1 4 :

齿

E

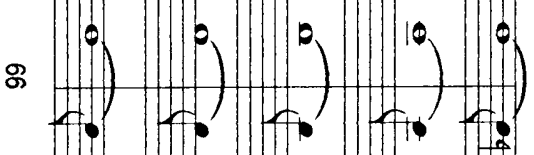

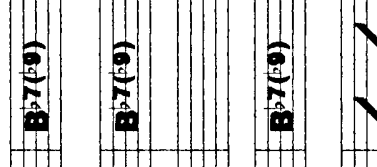

a o o m

H.

\& +

是

1. Ho $f^{\circ}$

a

6.

के

E

10.

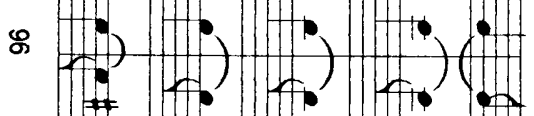

(ip) $f$ is

\&

疍

N. N:

表 $\frac{1}{6}$

E $E$ I

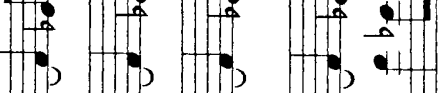

E

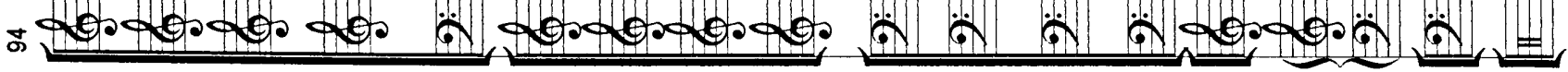




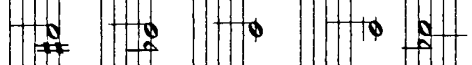

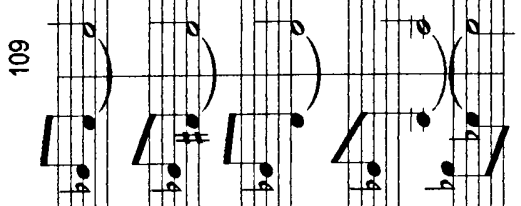

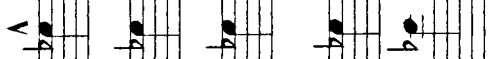

$\%$ \&

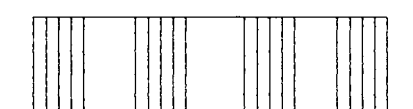

या याय $य ा \pi$

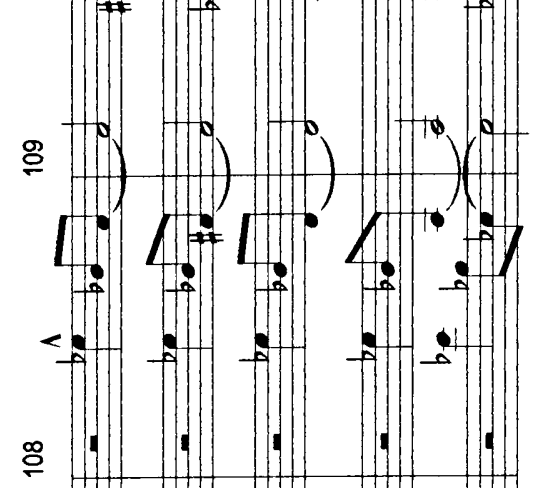

$2: \frac{1}{2}$

:

으

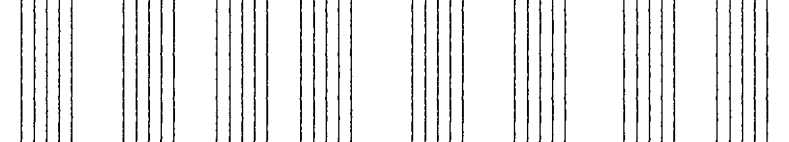

:

.

- $\because 1: 1: 1: 1: 1: 1$

\&

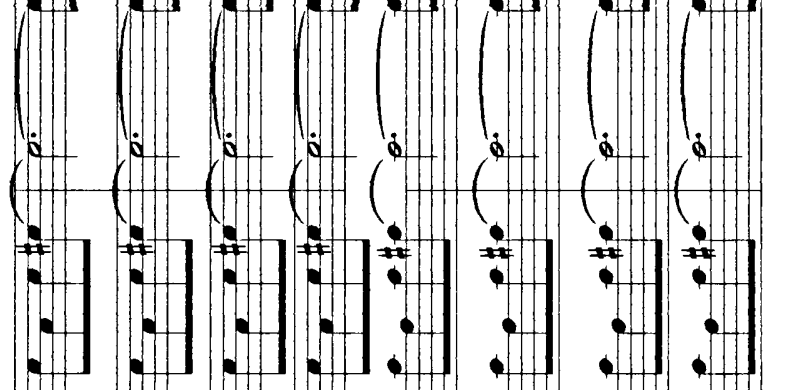

으

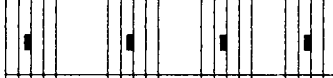

b

EN

-

으

a I 0 o

is

W

웅

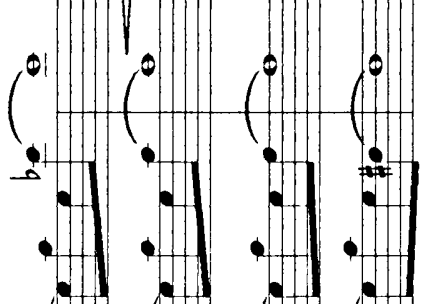

는

包

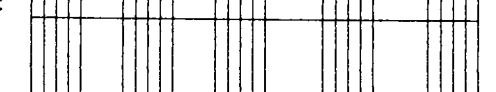

(1in

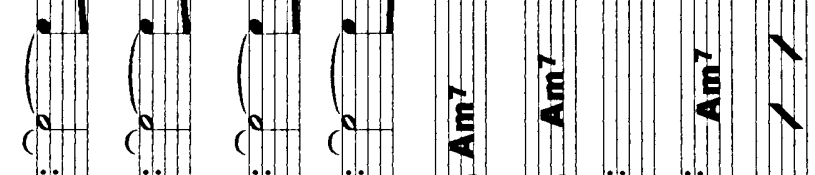

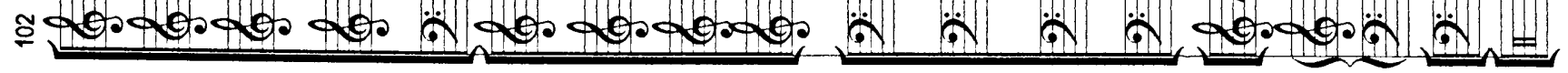


III: II: I: I: I:

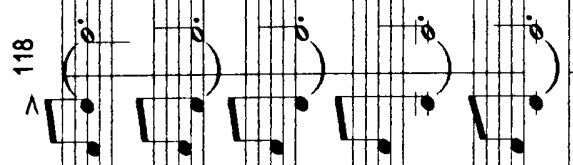

an: an: an: w:

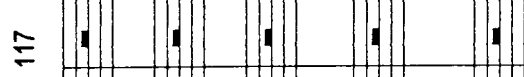

$\stackrel{2}{2} 11 \%$

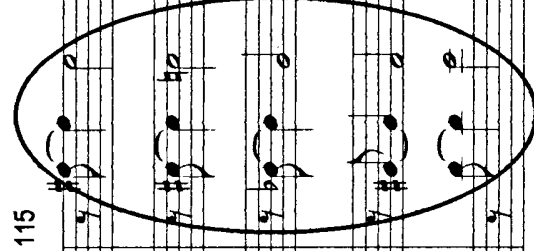

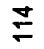

in: i: I:

$\stackrel{2}{2} \cdot 1.0$

$\cong$ 象

10 Ha

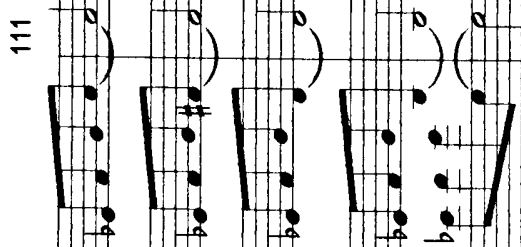

110

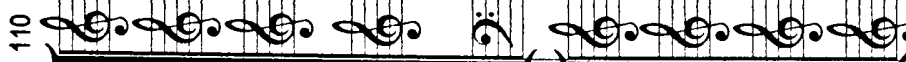

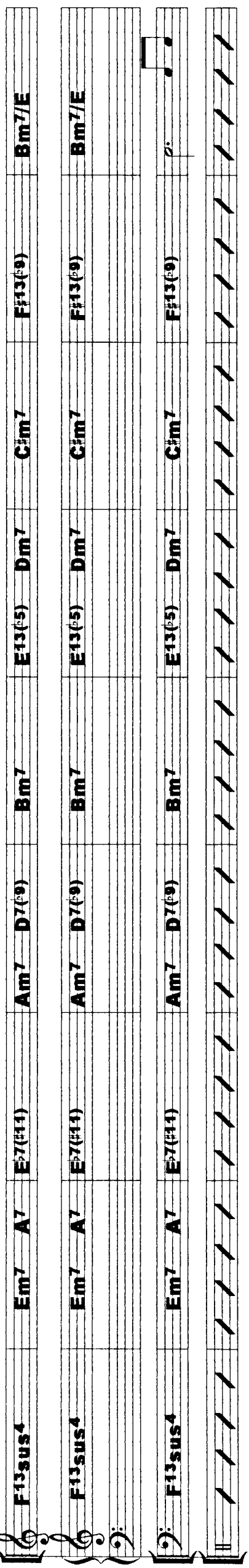


$\because 1: 1: \mathbb{:}: \mathbb{:}:$

$\approx(0-\because \%$ is

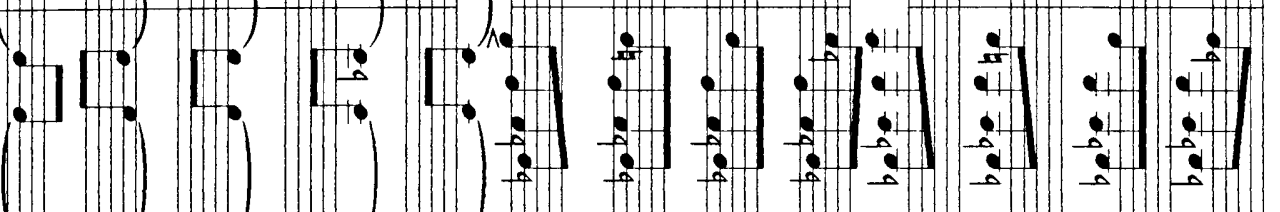

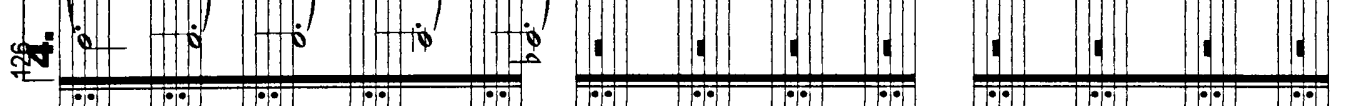

$\frac{-10}{2}$

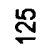

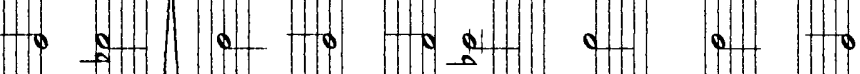

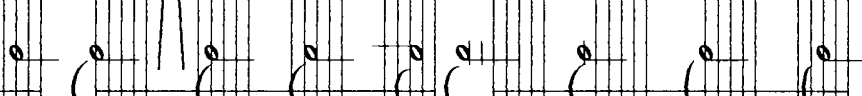

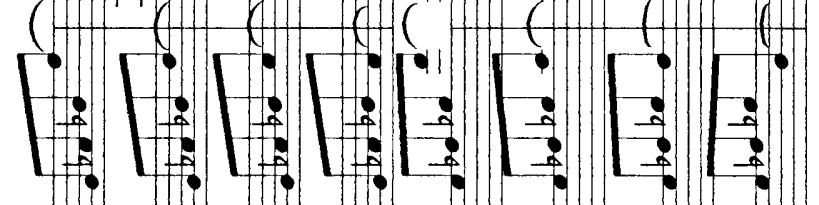

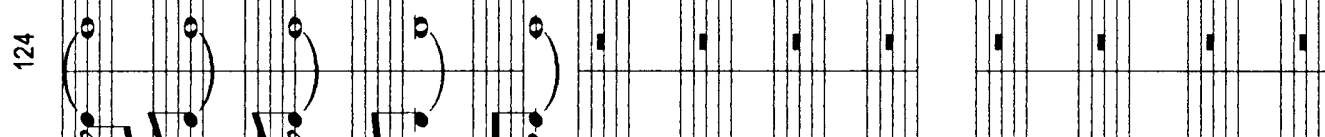

部

$: 11:$ : $:$ :

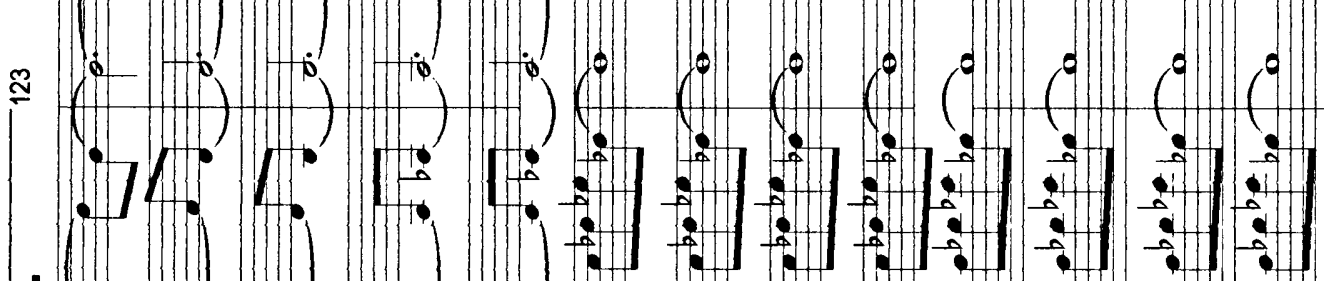

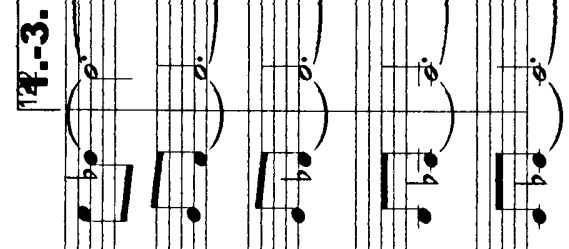

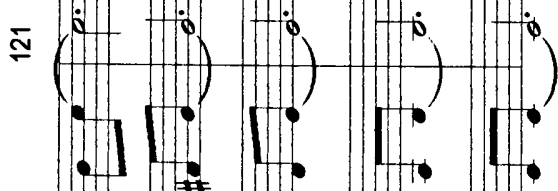

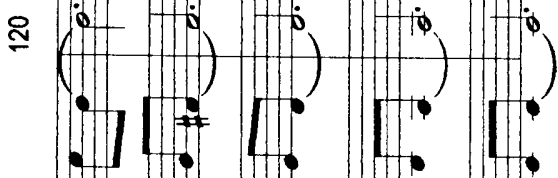

Q $9+$ is

. . . .

倍

(n)

崖

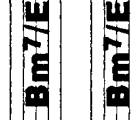

$\stackrel{m}{\frac{T}{*}}$

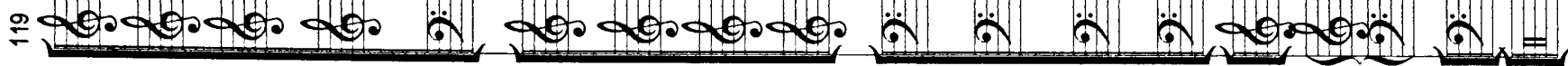


PFi

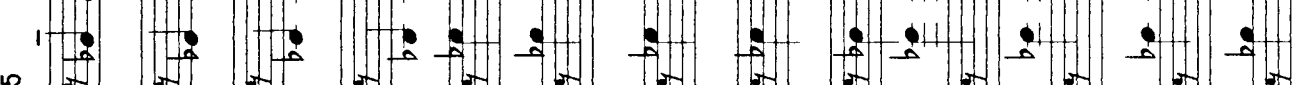

邑

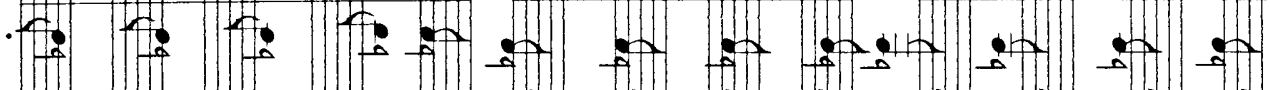

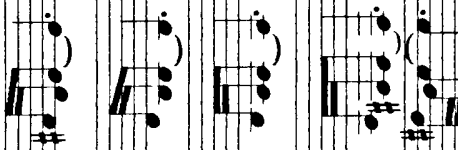

:

11: $:$

$\stackrel{\check{g}}{\underline{m}}$

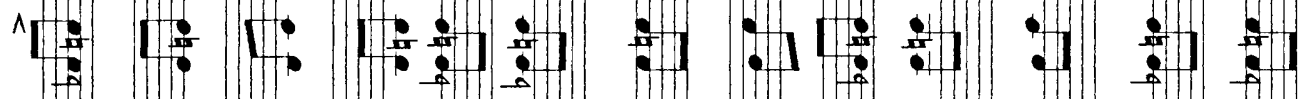

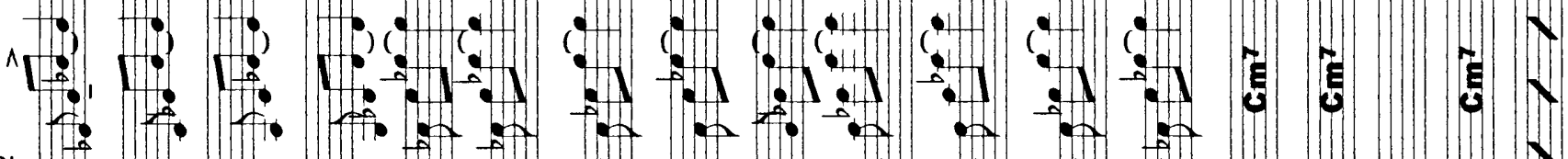

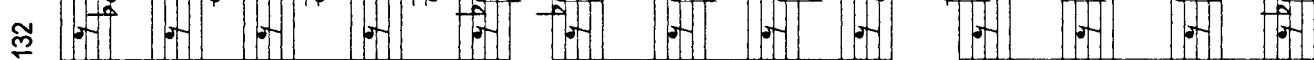

党

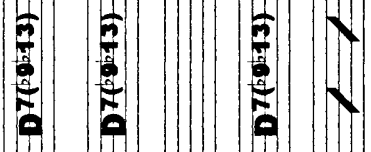

E

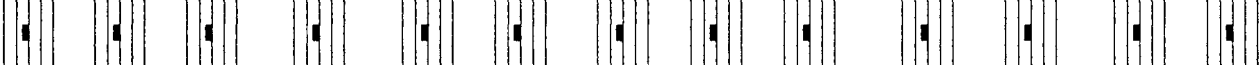

12 का

旁

5 (1)

-

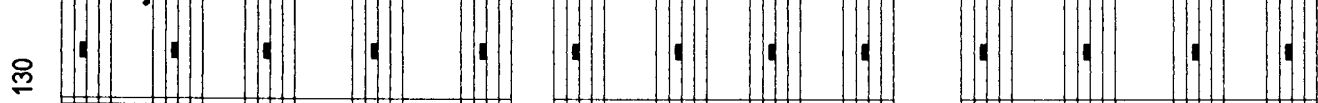

(5)

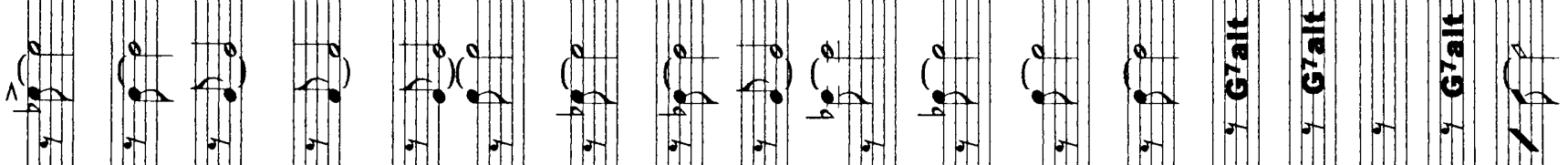

2.....................

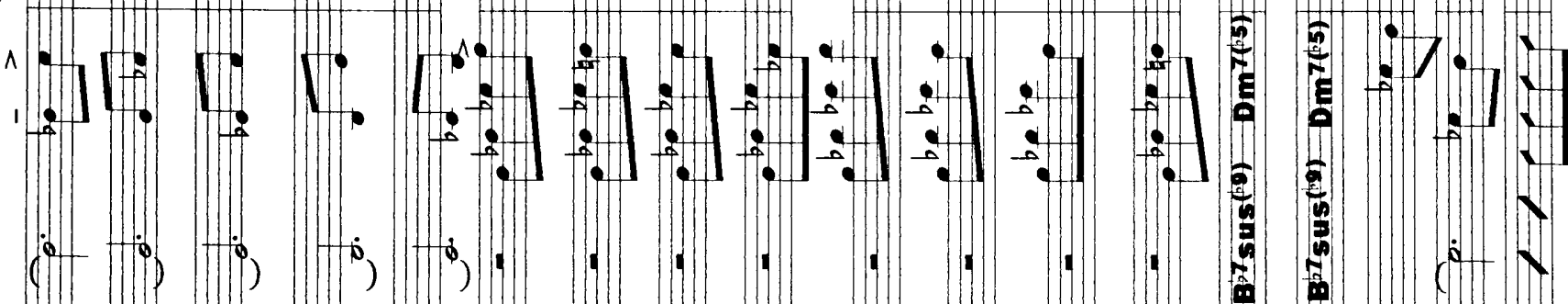

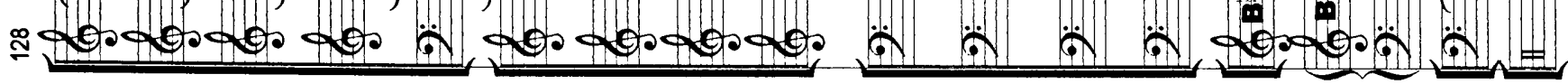




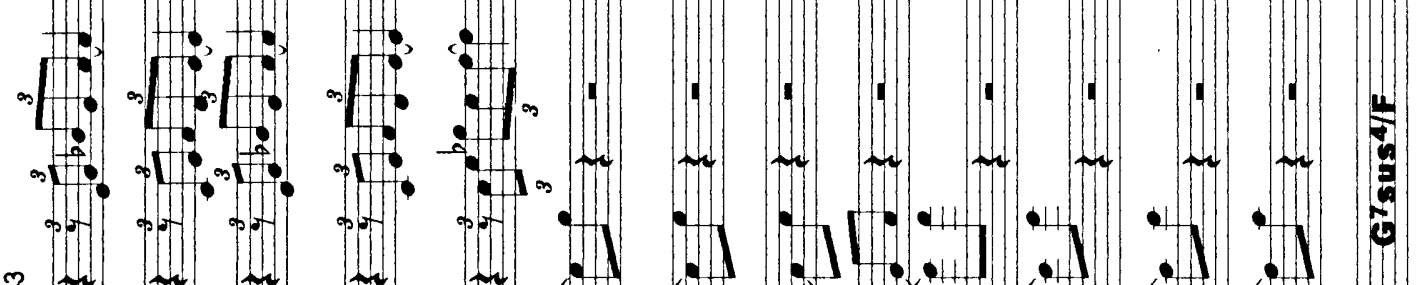

\% 7 tw

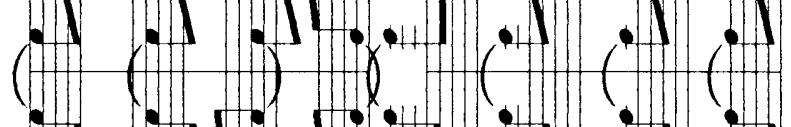

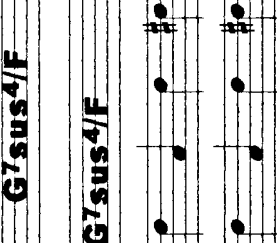
$: 1: 1: \div: 1: 1: 1: 1$

:

¥

6 Ib Ulo

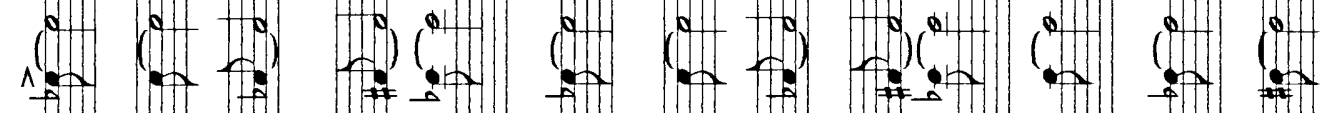

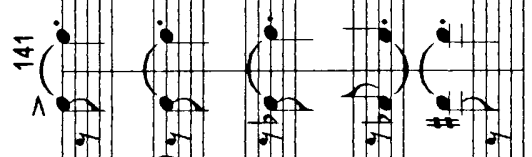

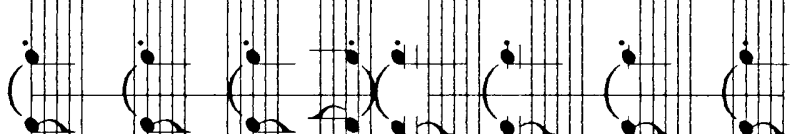

$\div \quad \frac{1}{5}$

: $5: \div$

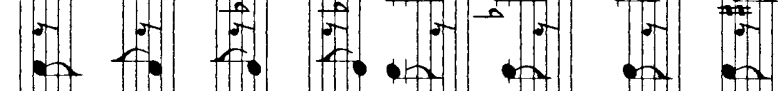

$>:: ;:$

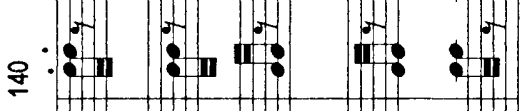

$\therefore: \therefore:$ : : : :

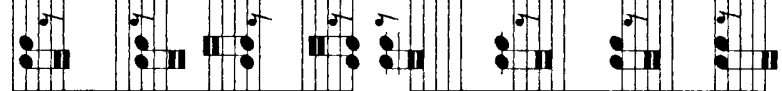

要

访:

$\therefore$

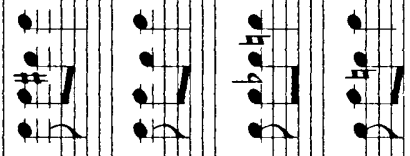

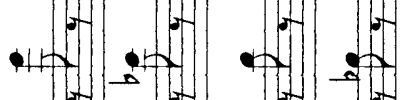

ถ้ํำ

( )

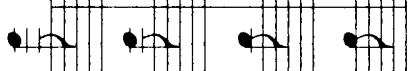

$\sqrt{ }$

证:

$\because \div \div \div: \div$

: : :

$\because+\cdots$

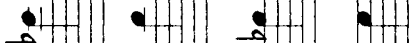

$+1:-1:-1:-1.10$

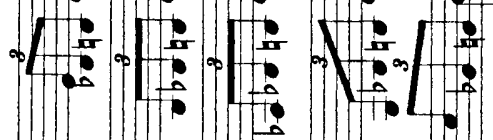

c.

Q. of 2 a

的

:

:

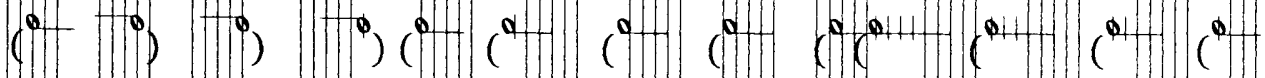

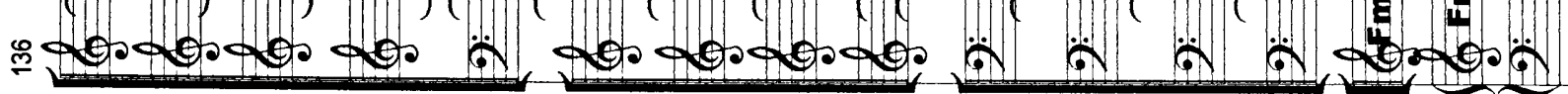


(III III)

- $\rightarrow$,

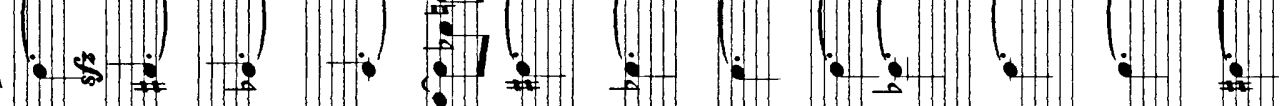

.

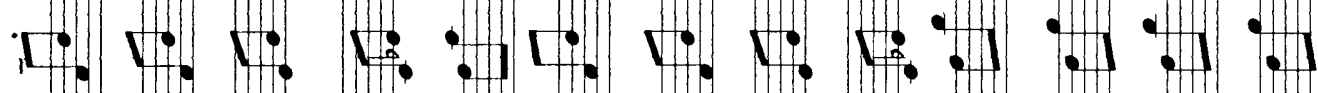

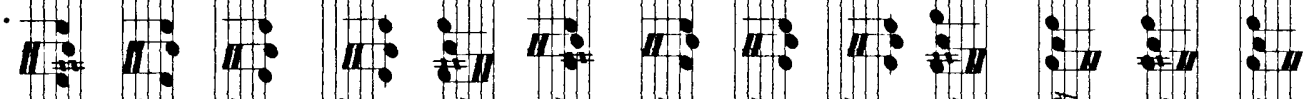

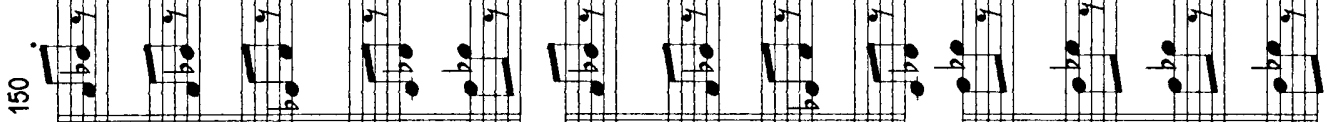

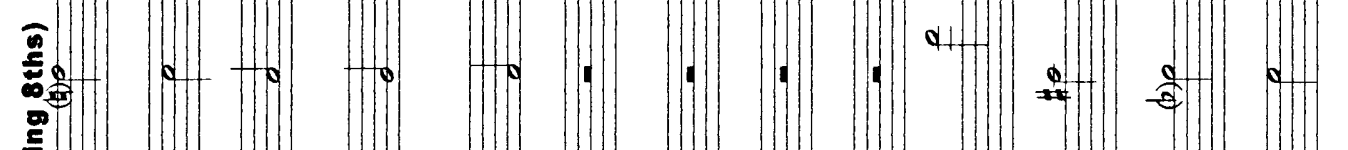

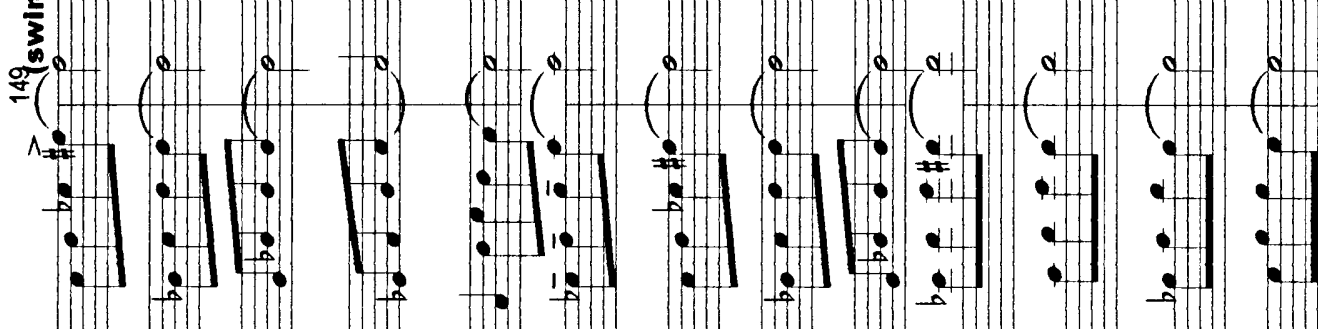

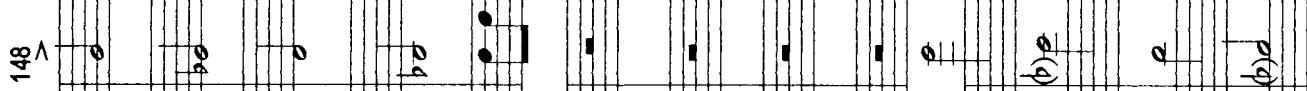

या

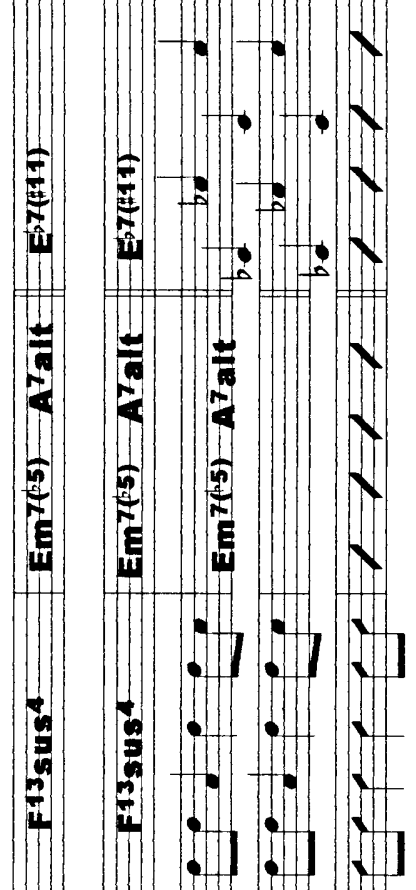

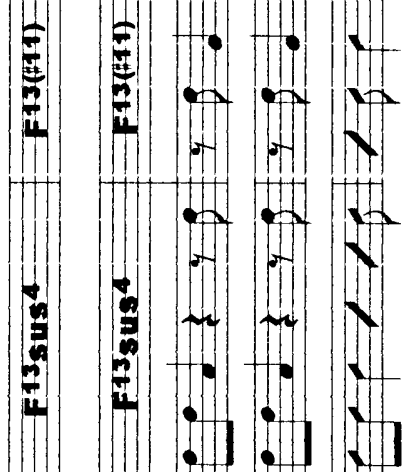

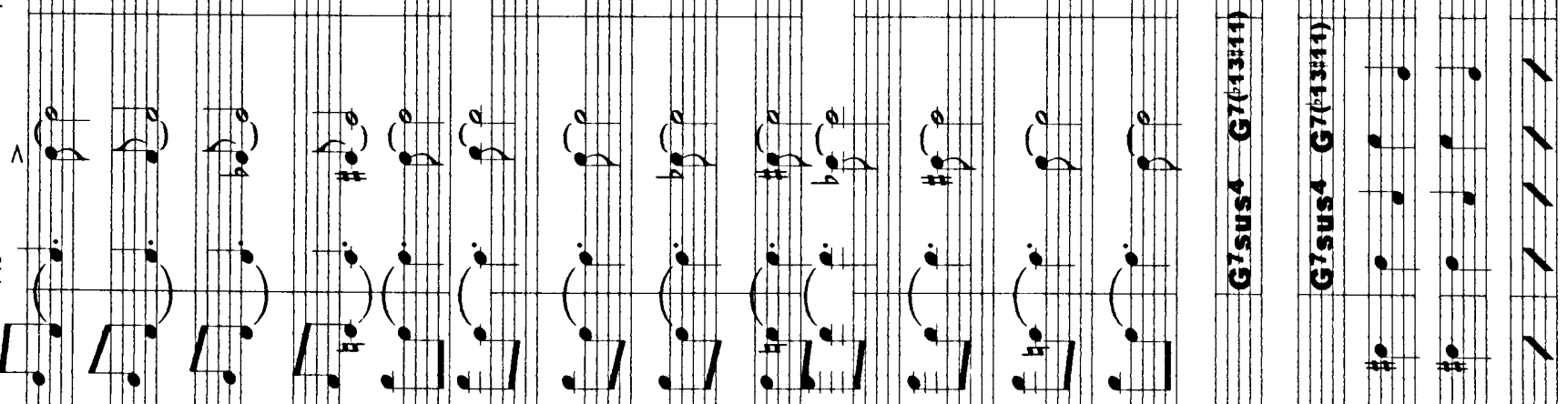

(10.2) 2
2

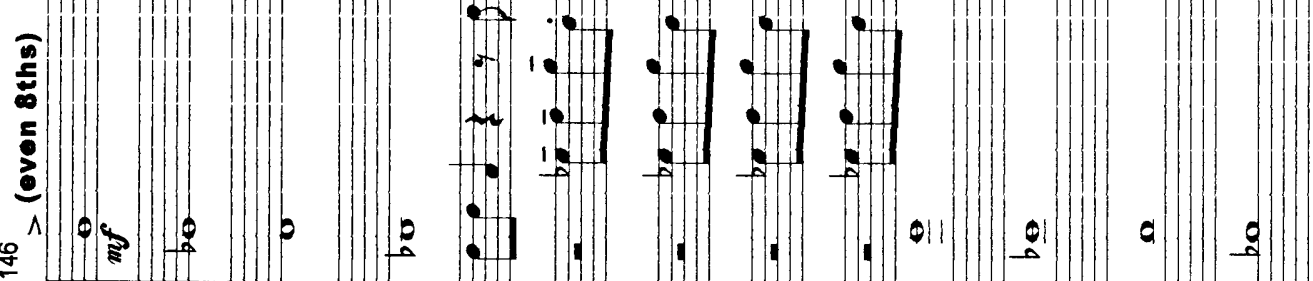

: $:=2$

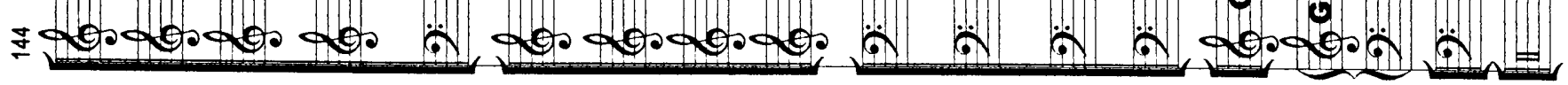




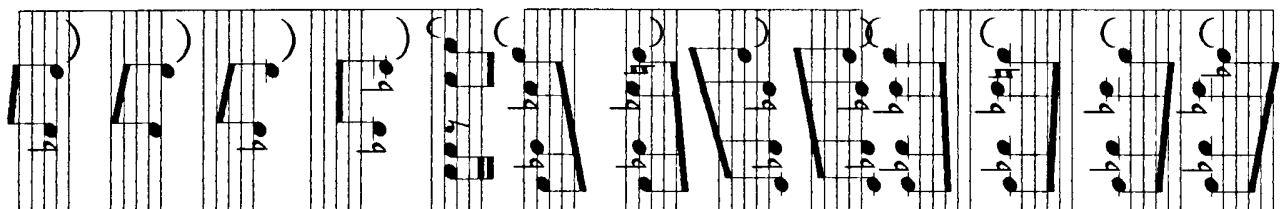

I3)

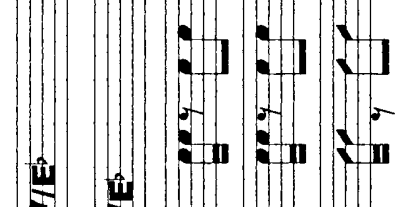

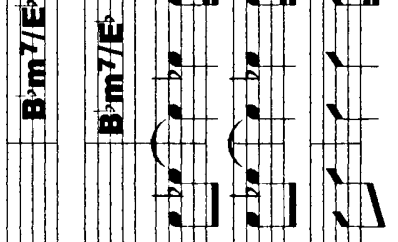

I. I. If I: : $\ldots \ldots \ldots$

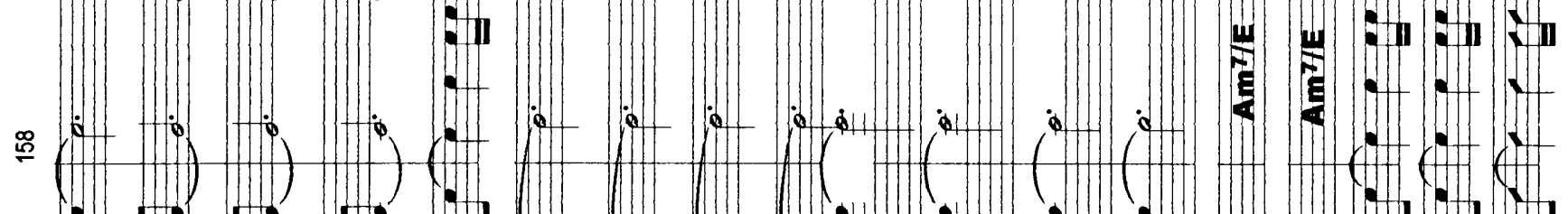

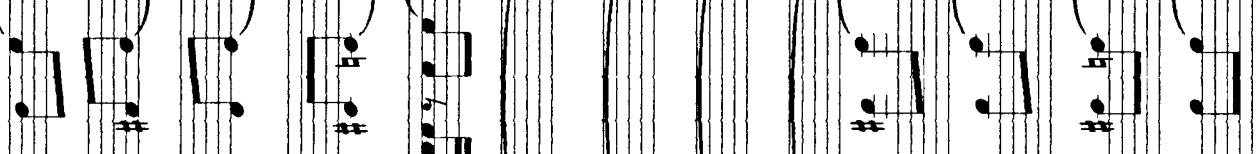

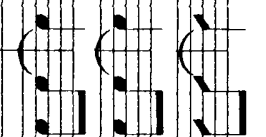

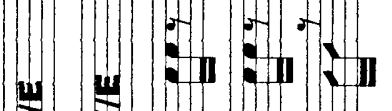

5 5.

盗: : : :

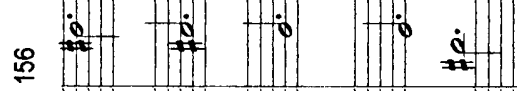

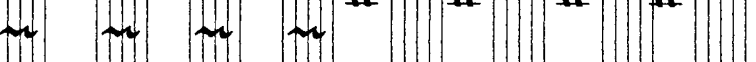

:

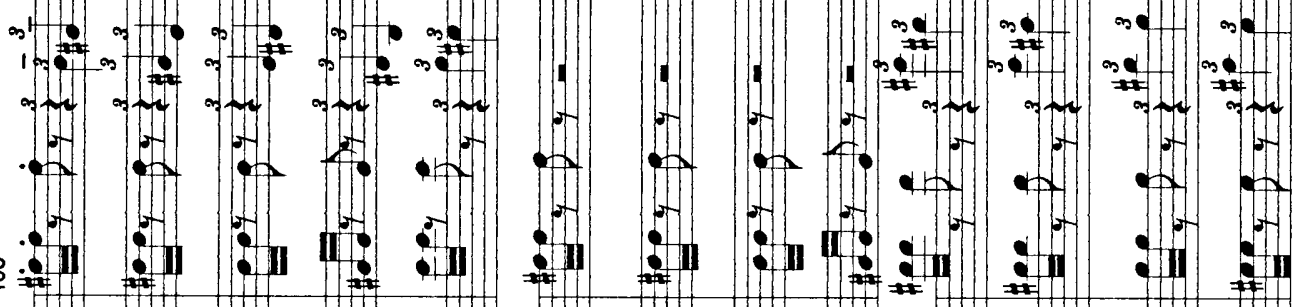

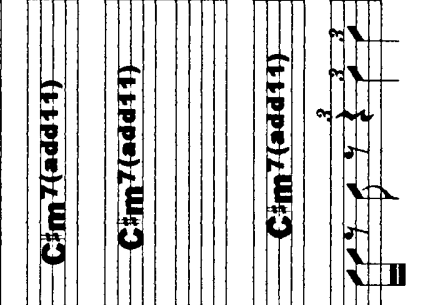

- st to

$\therefore(6)$

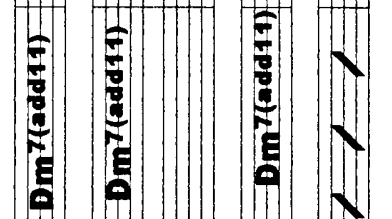

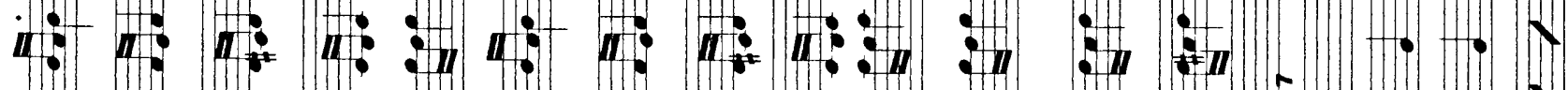

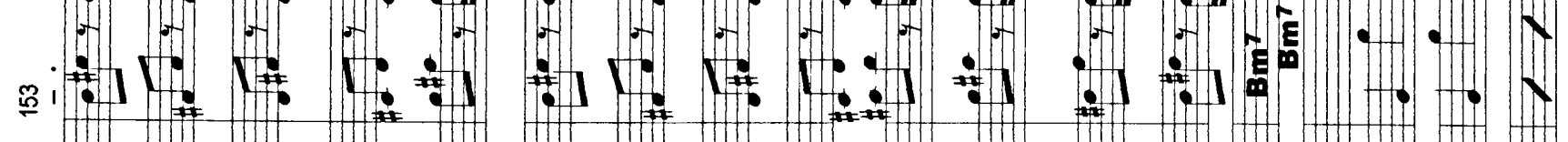

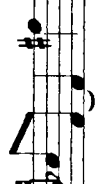

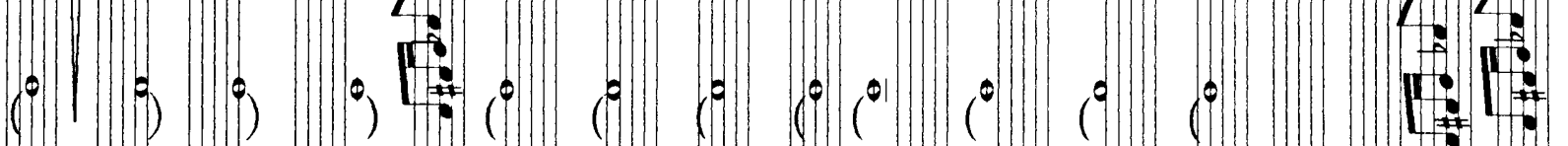

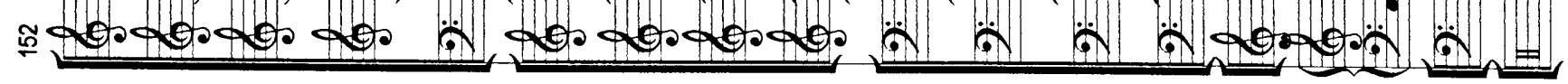




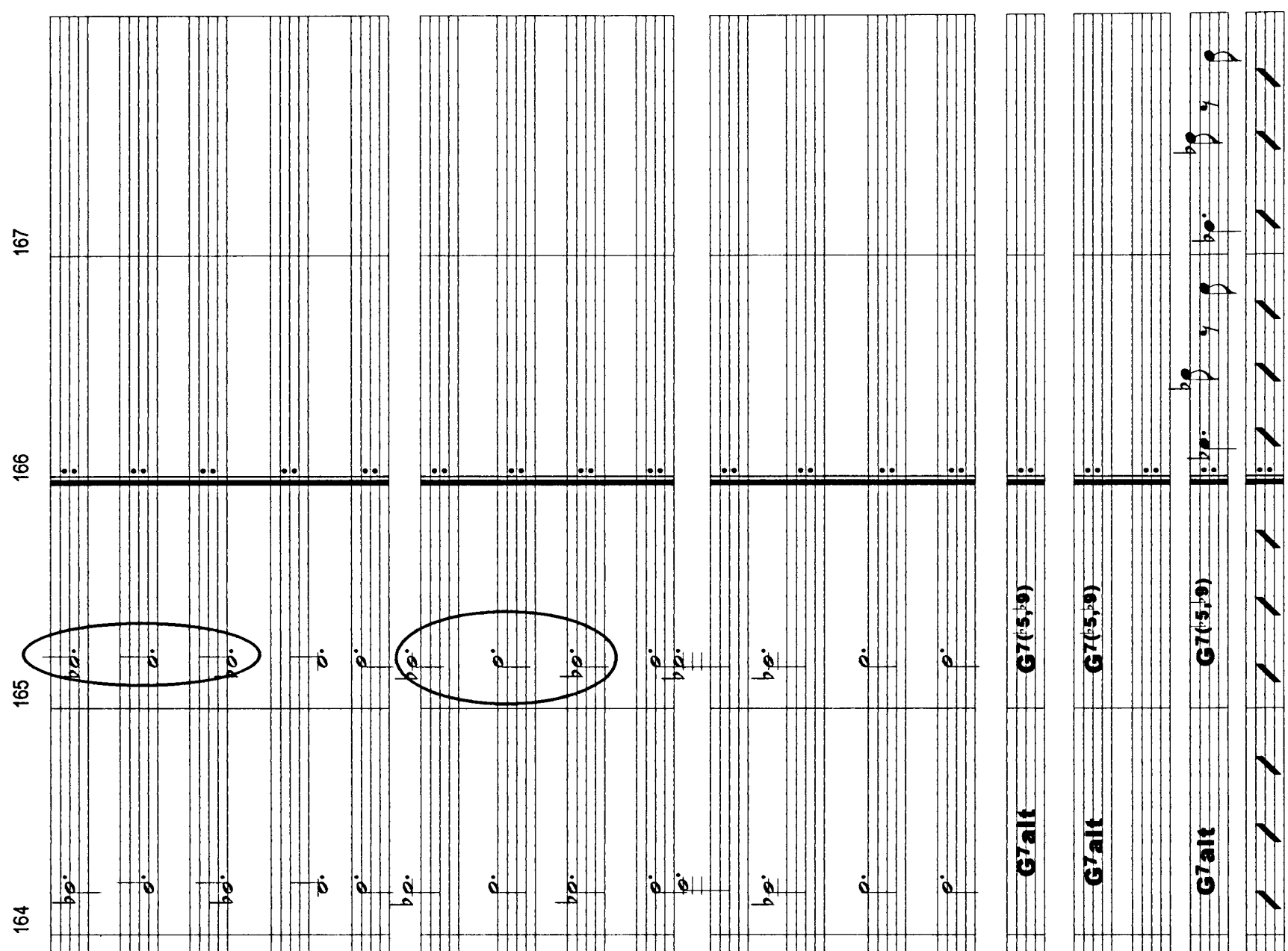

$\wedge$ fo

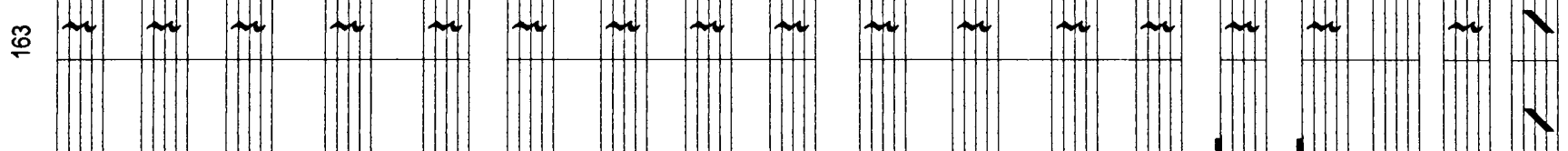

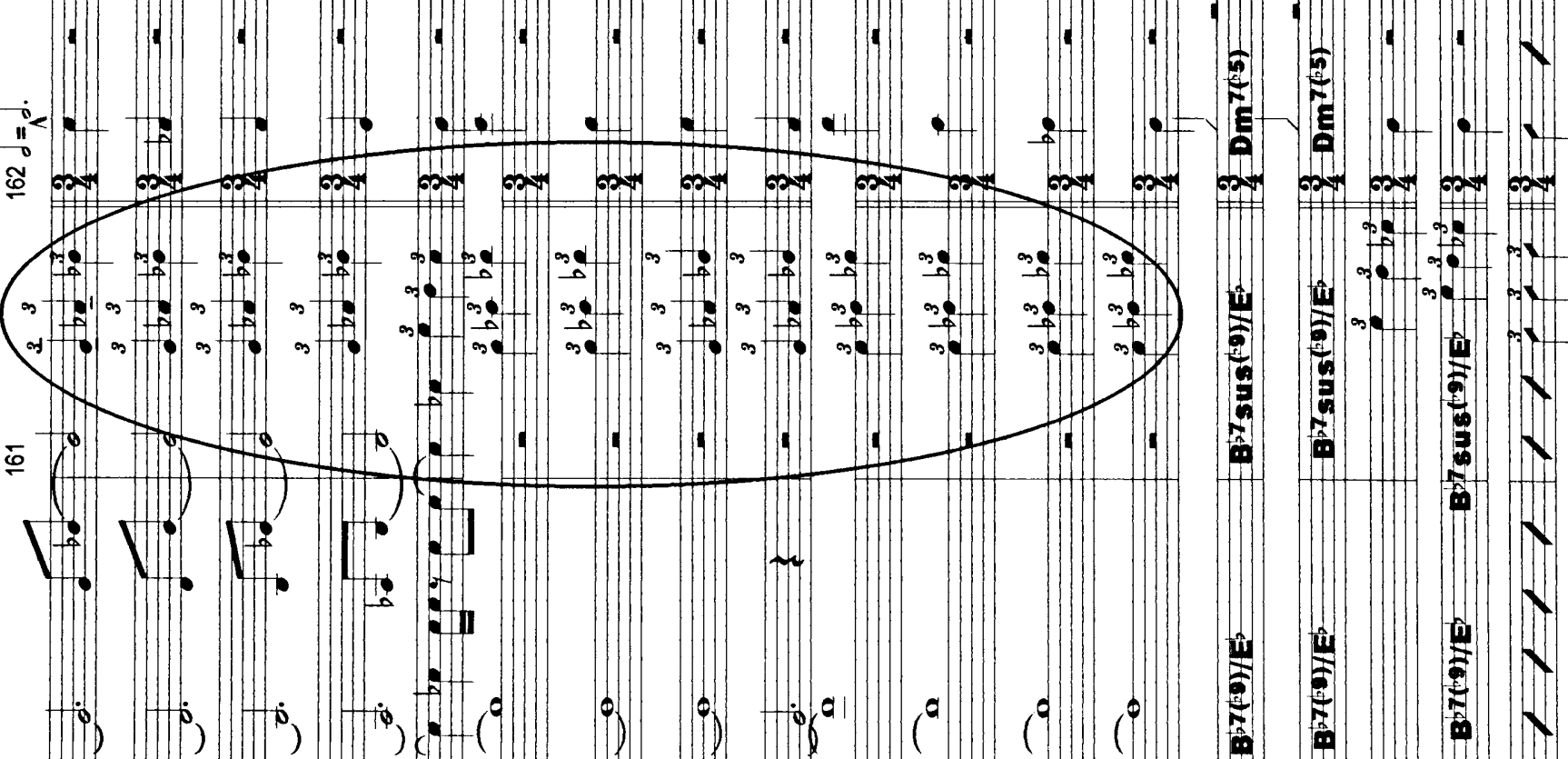

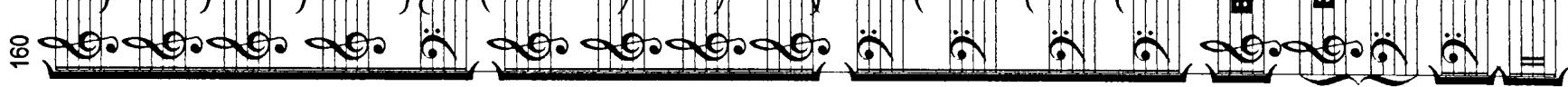



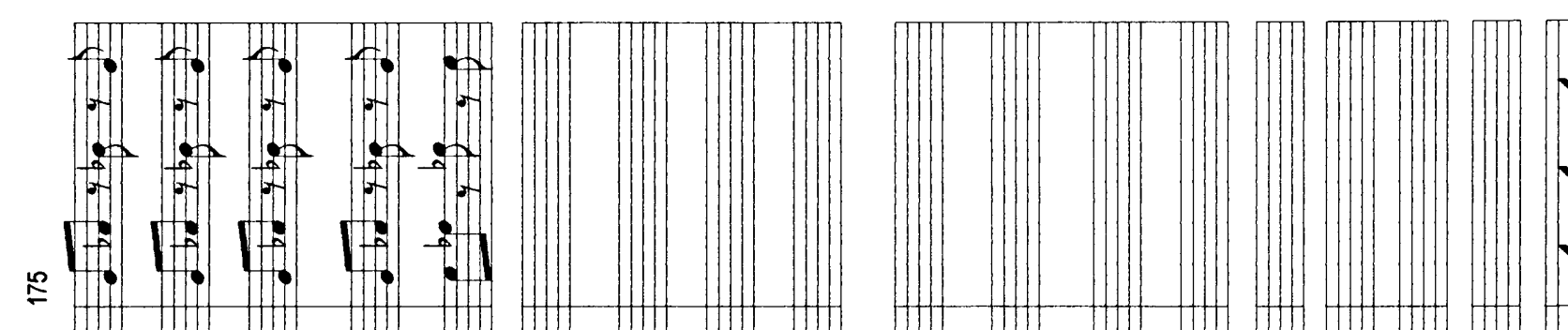

$\sqrt{1}$

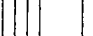

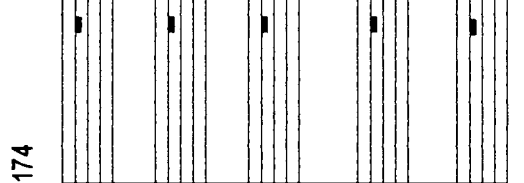

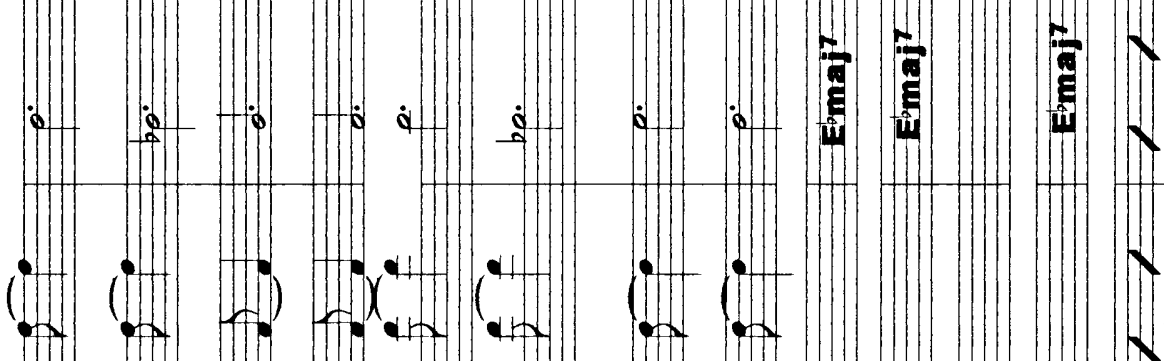

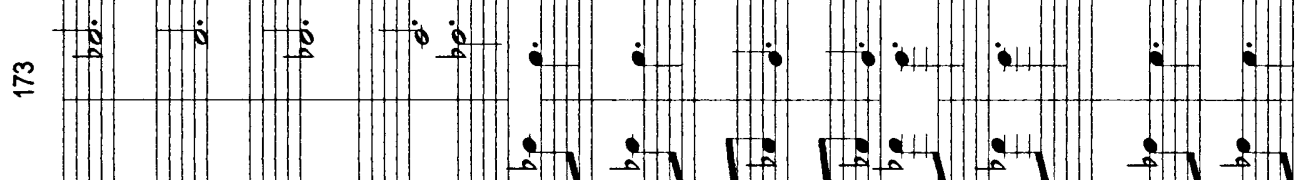

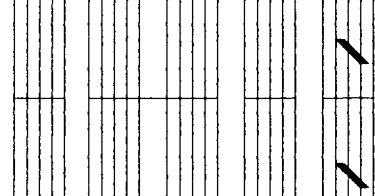

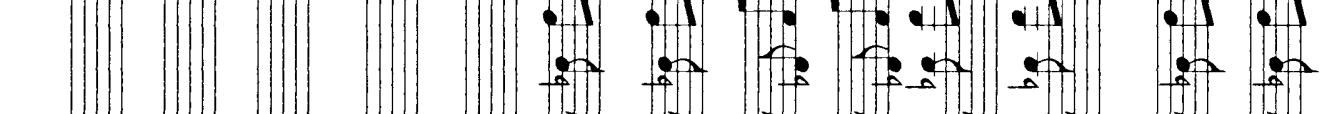

₹

急

W

$\Sigma$ If

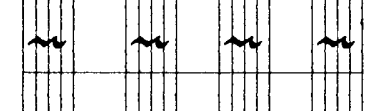

H fut

$\stackrel{0}{\approx}$
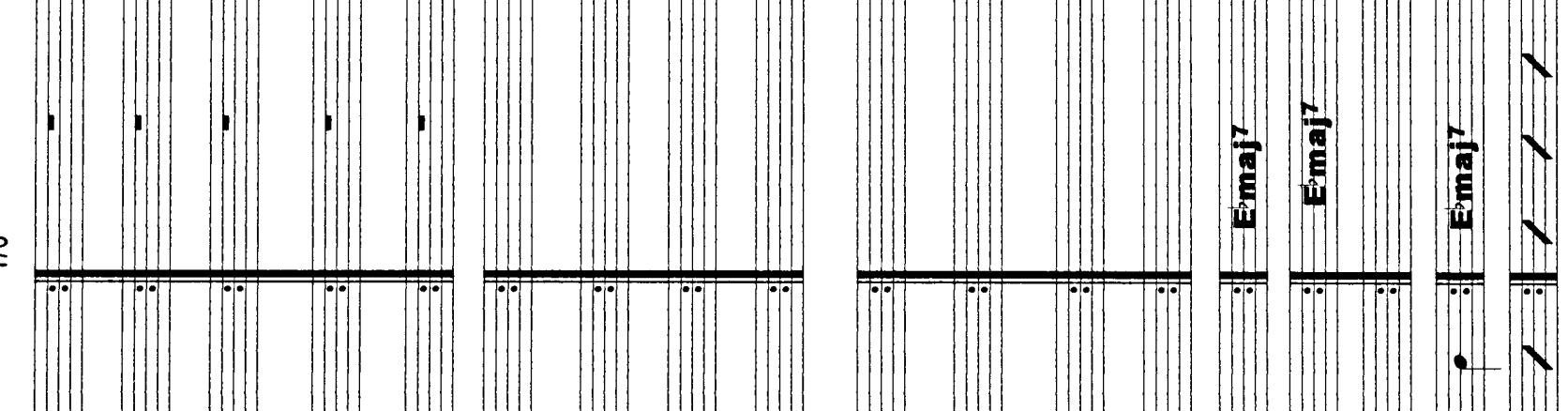

2N

\&
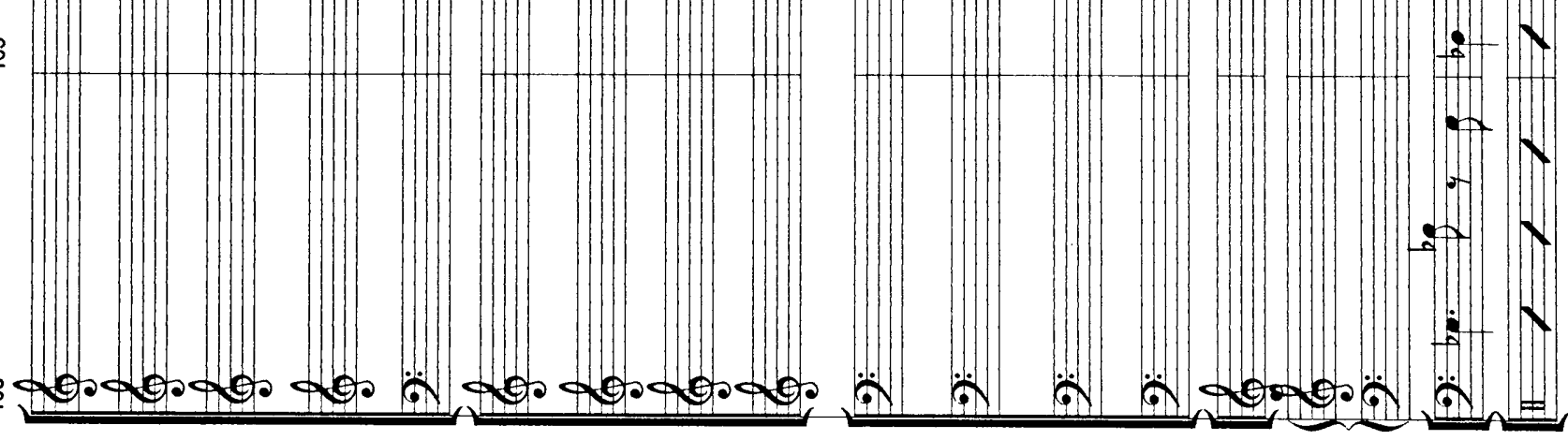

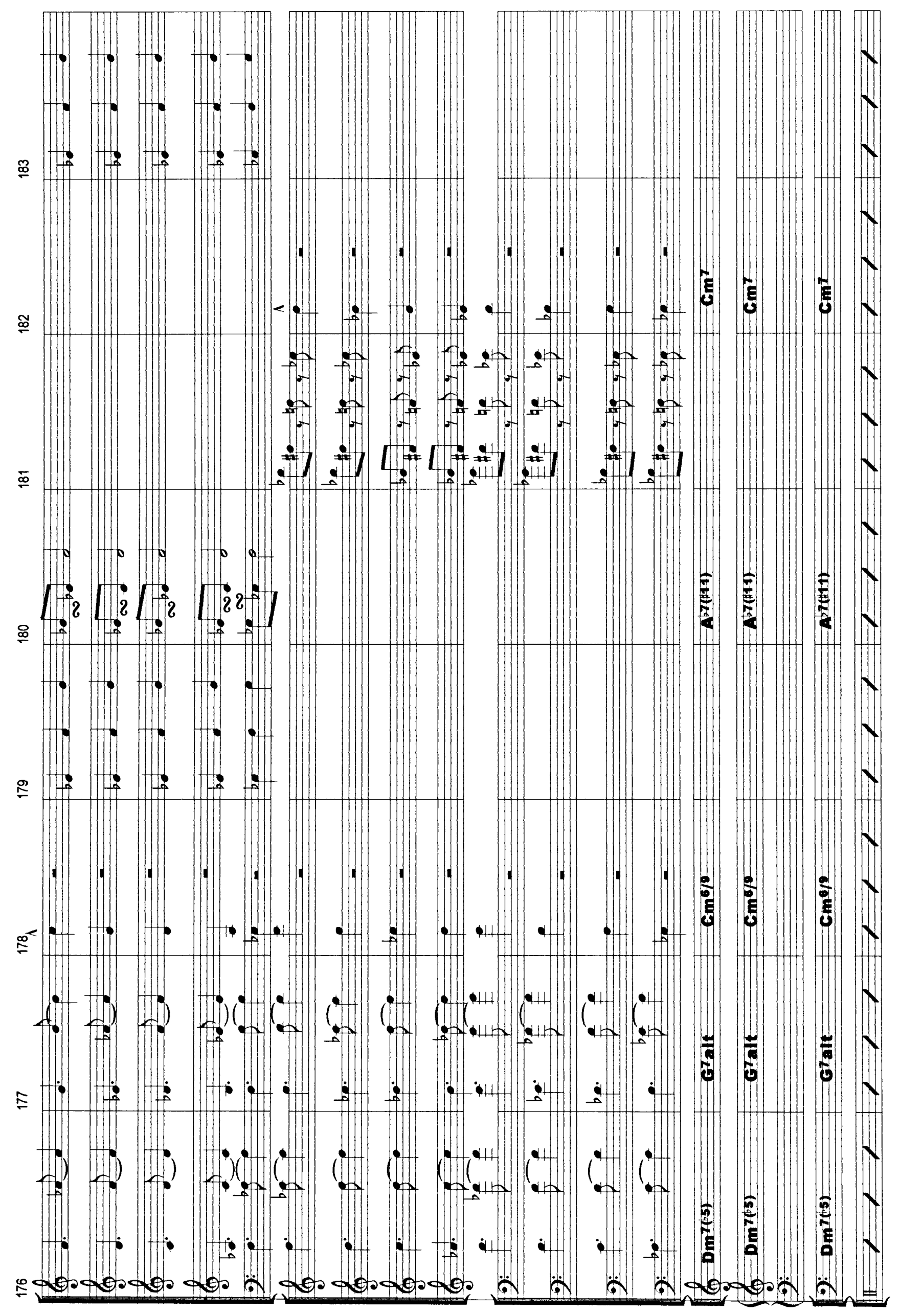


$$
\begin{aligned}
& \text { the }
\end{aligned}
$$

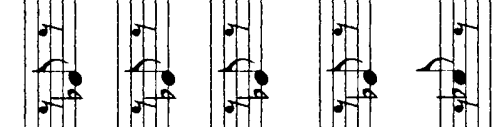

$$
\begin{aligned}
& 519: 19: 5: 11
\end{aligned}
$$$$
\text { in }
$$

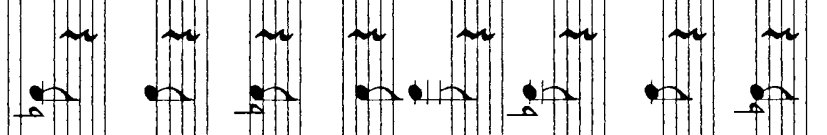$$
\text { 옹 }
$$

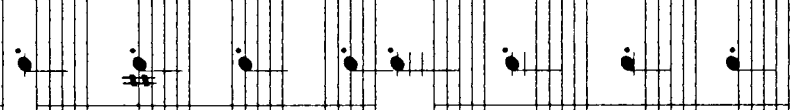

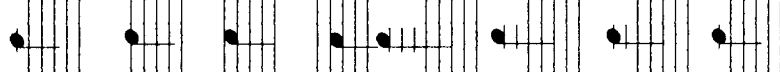

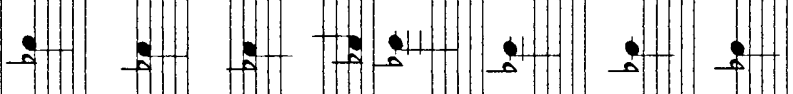

$\stackrel{\mathscr{\Xi}}{\mathscr{0}}$

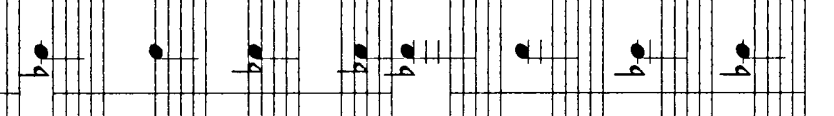

6.

Y

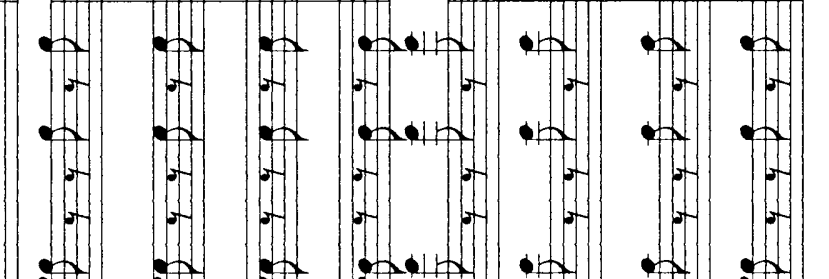

$\stackrel{\infty}{\infty}$

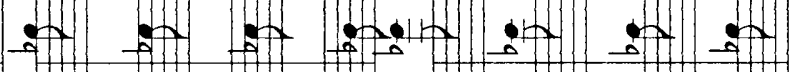

E

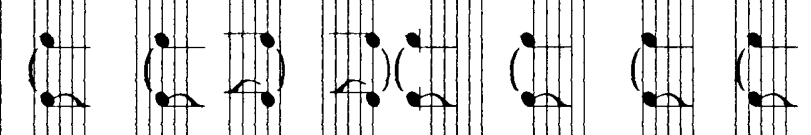

क्ष

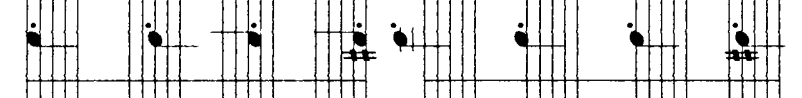

(.)

$\stackrel{\infty}{\varpi}$

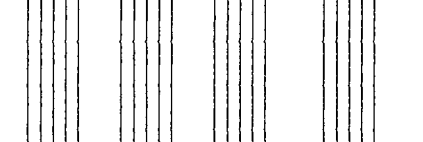

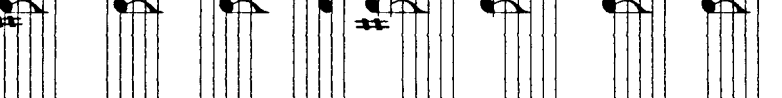

.

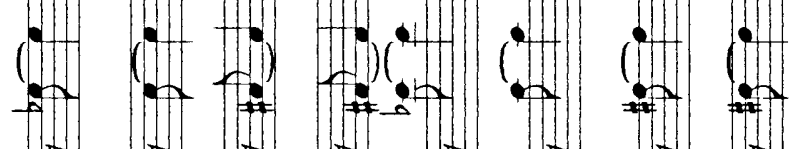

$\stackrel{\infty}{\infty}$

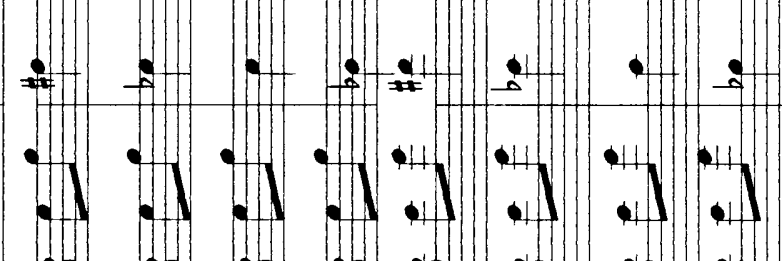

蛭

$\frac{1}{8}$

m 11 m

if

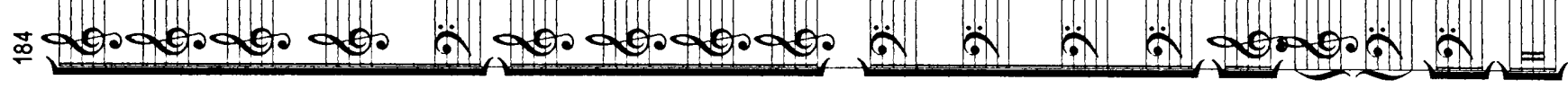


तir: $\pi: \pi: \pi$

g

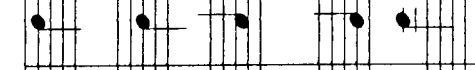

- $\rightarrow+\cdots$

1. $\left.f \circ f_{0}\right)(t)$

$\stackrel{\infty}{\circ}$

a.

(5)

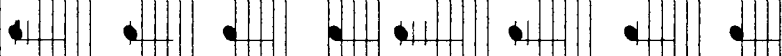

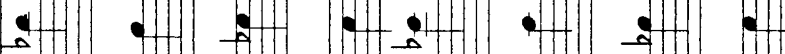

के

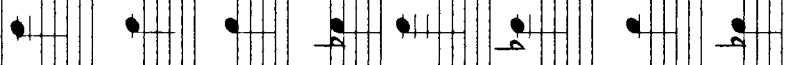

(-) (1) (1) (1) (1)

$\stackrel{9}{9}$

$\therefore$ : $: 0.11$ : $: 1$

(6)

要

용

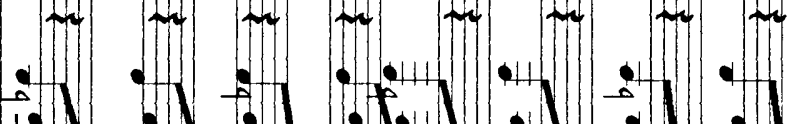

(2)

E)

号

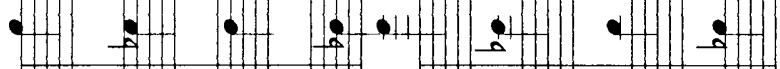

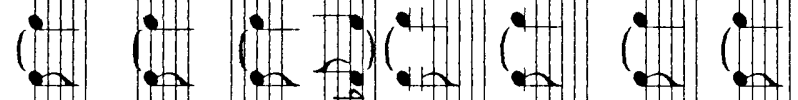

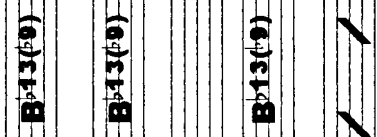

\%

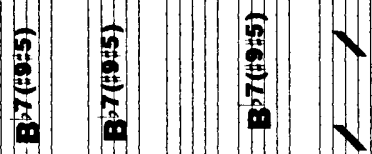

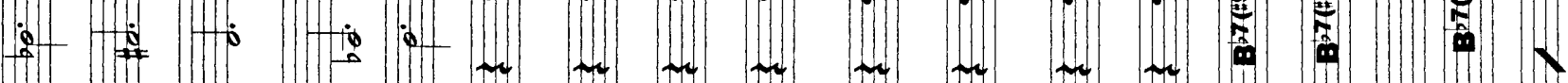

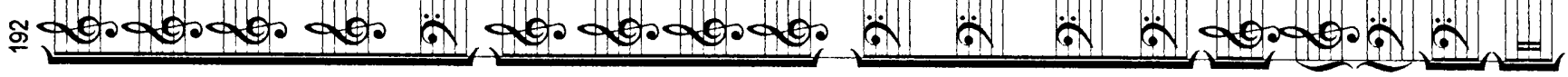


㝏

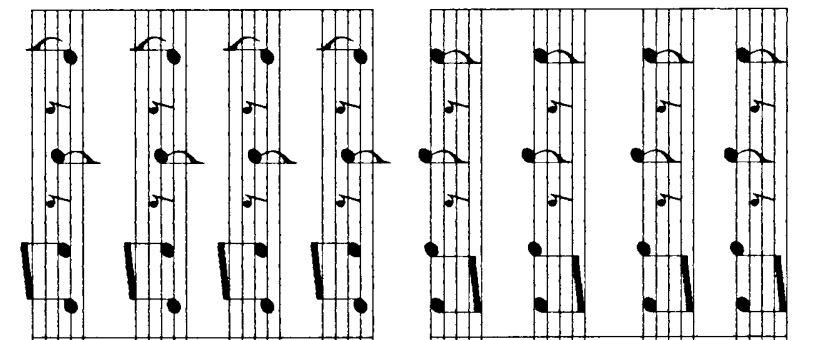

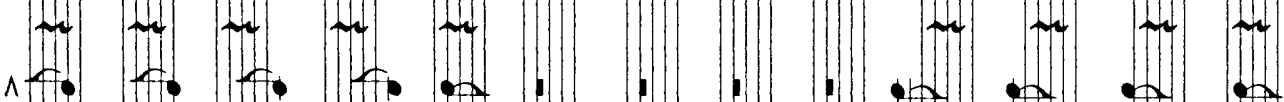

$\stackrel{\circ}{\circ}$

:
E

$\frac{5}{6}$

1: $1:$ : : : : :1

? ? ? : ? ?

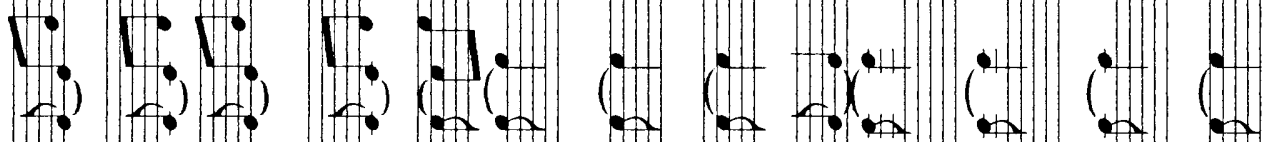

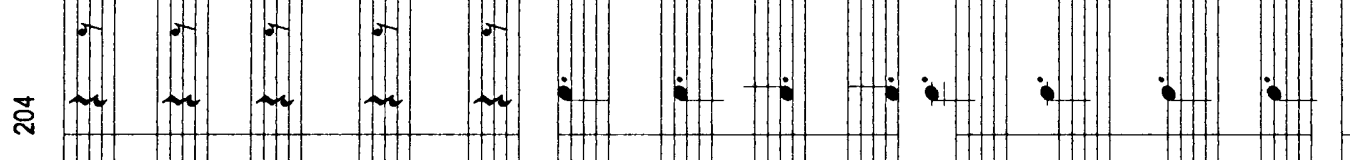

$:(1)$

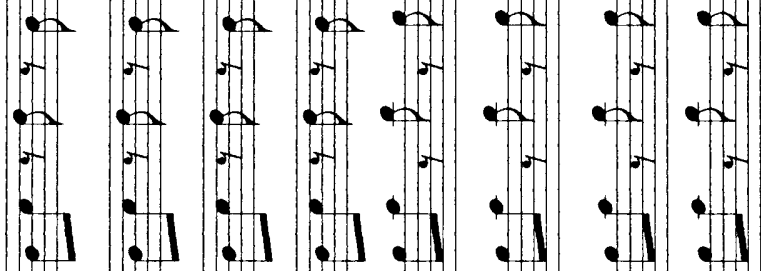

ౖ̊

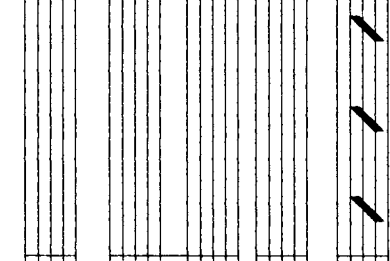

(

$: 0.0$ a

ธ్

1. $+\infty$

1.) $\rightarrow$ क्षे

$\overline{2}+\infty=$

高

-. $+4 \div$

(1)

a $\frac{6}{2}$

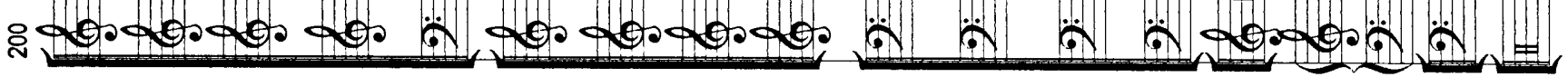




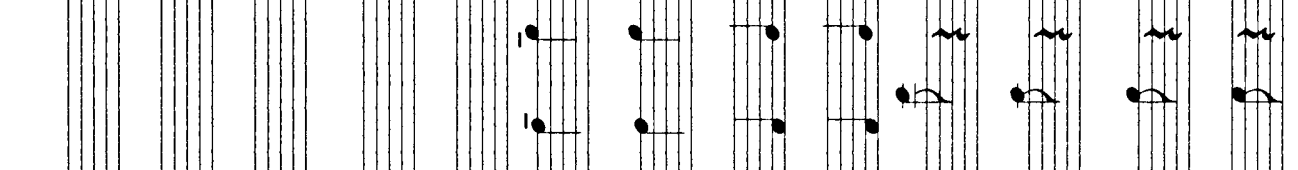

$\stackrel{m}{\sim} \|$ Un
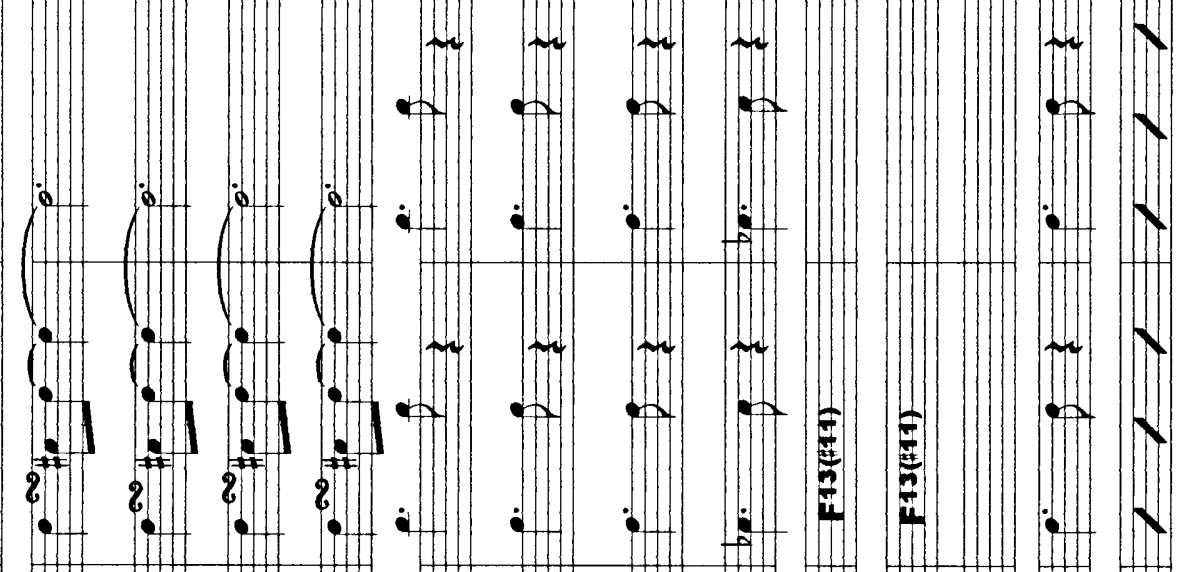

.

产

- N

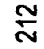

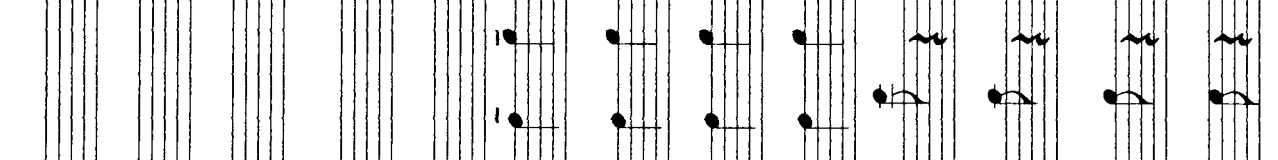

- $N$

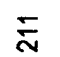

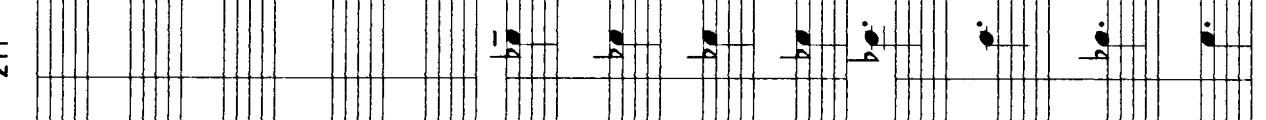

-.

N

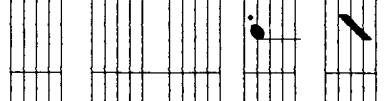

일.

$$
\text { m. } m
$$$$
\text { W }
$$

- 2.0

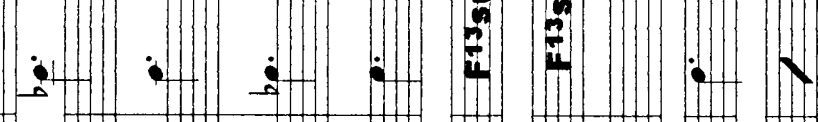
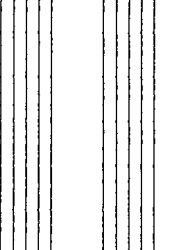


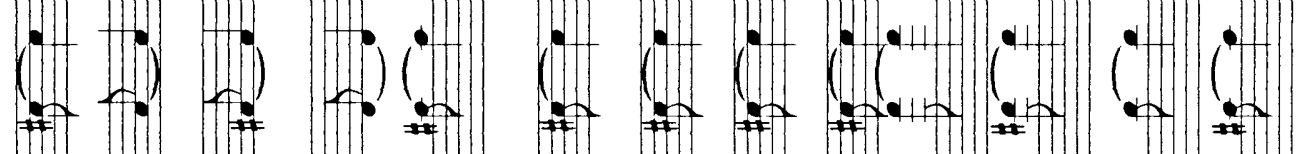
$\therefore: \div: \div: \div: \div \div:$

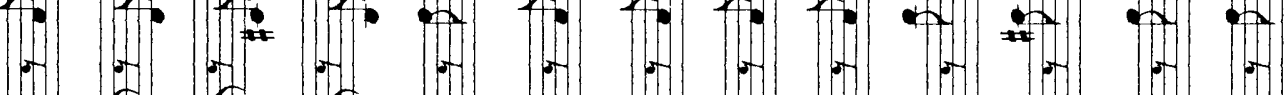

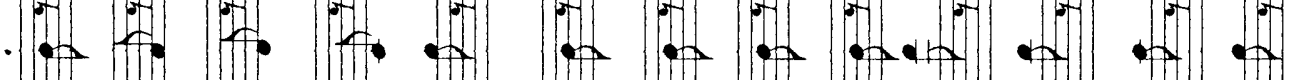

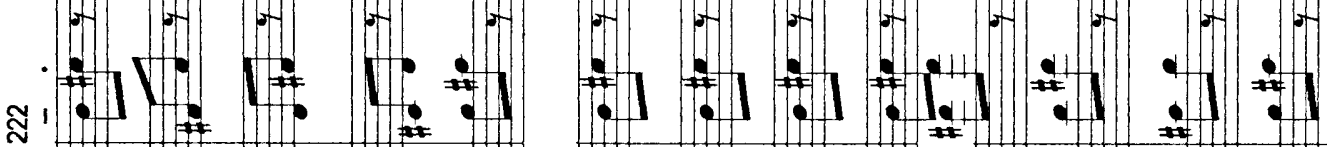

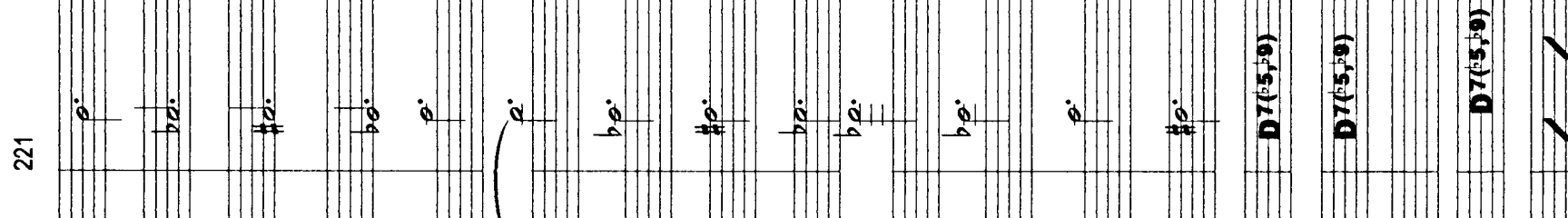

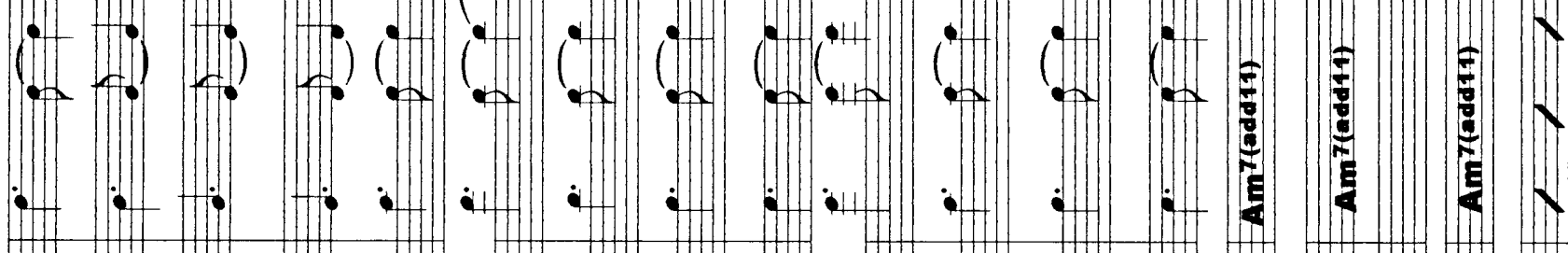

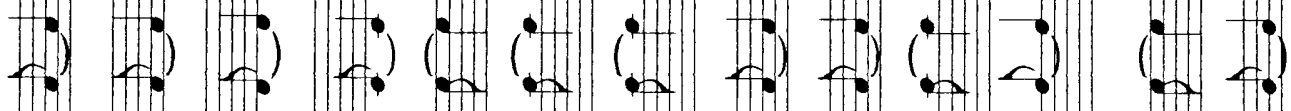

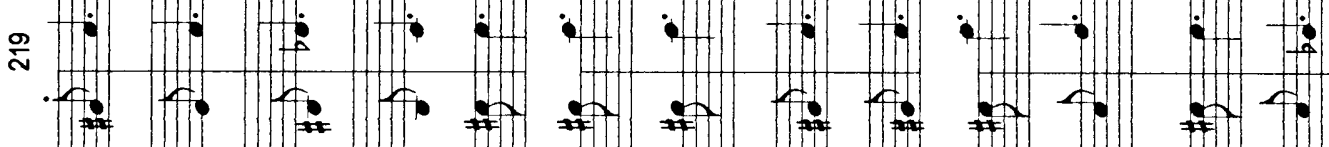

H.

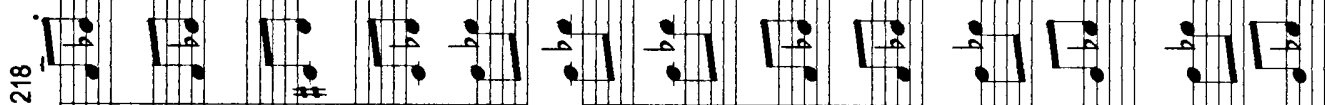

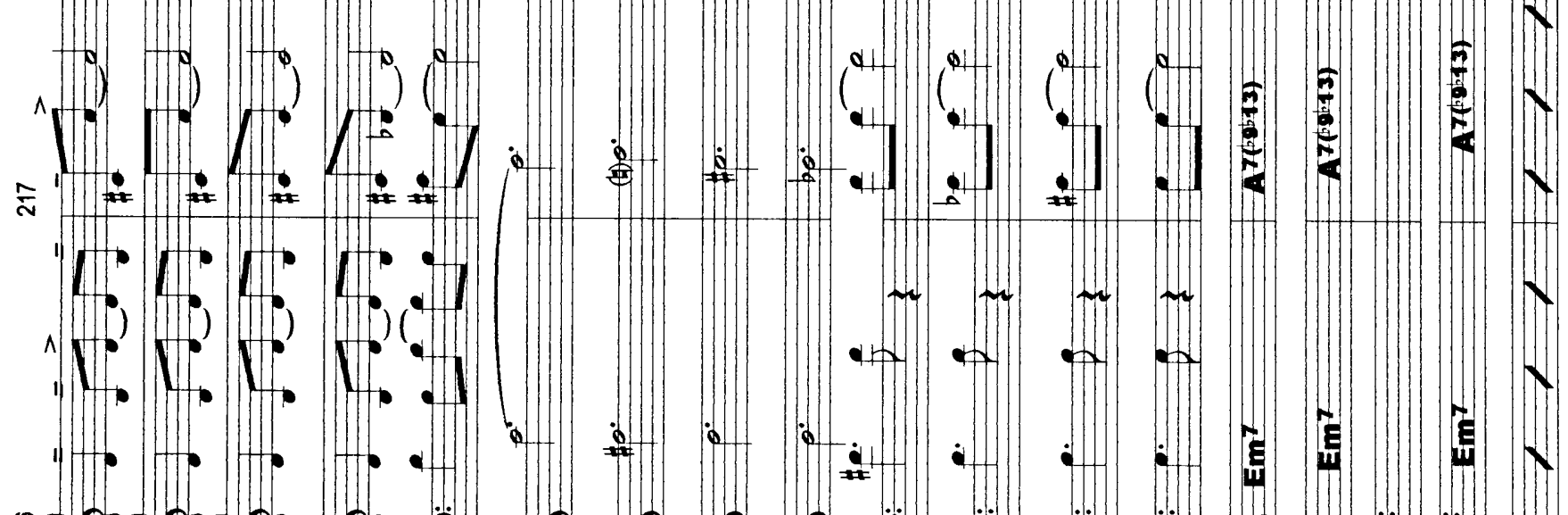

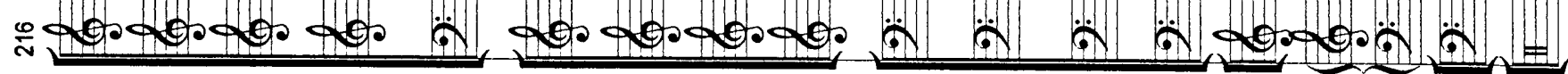



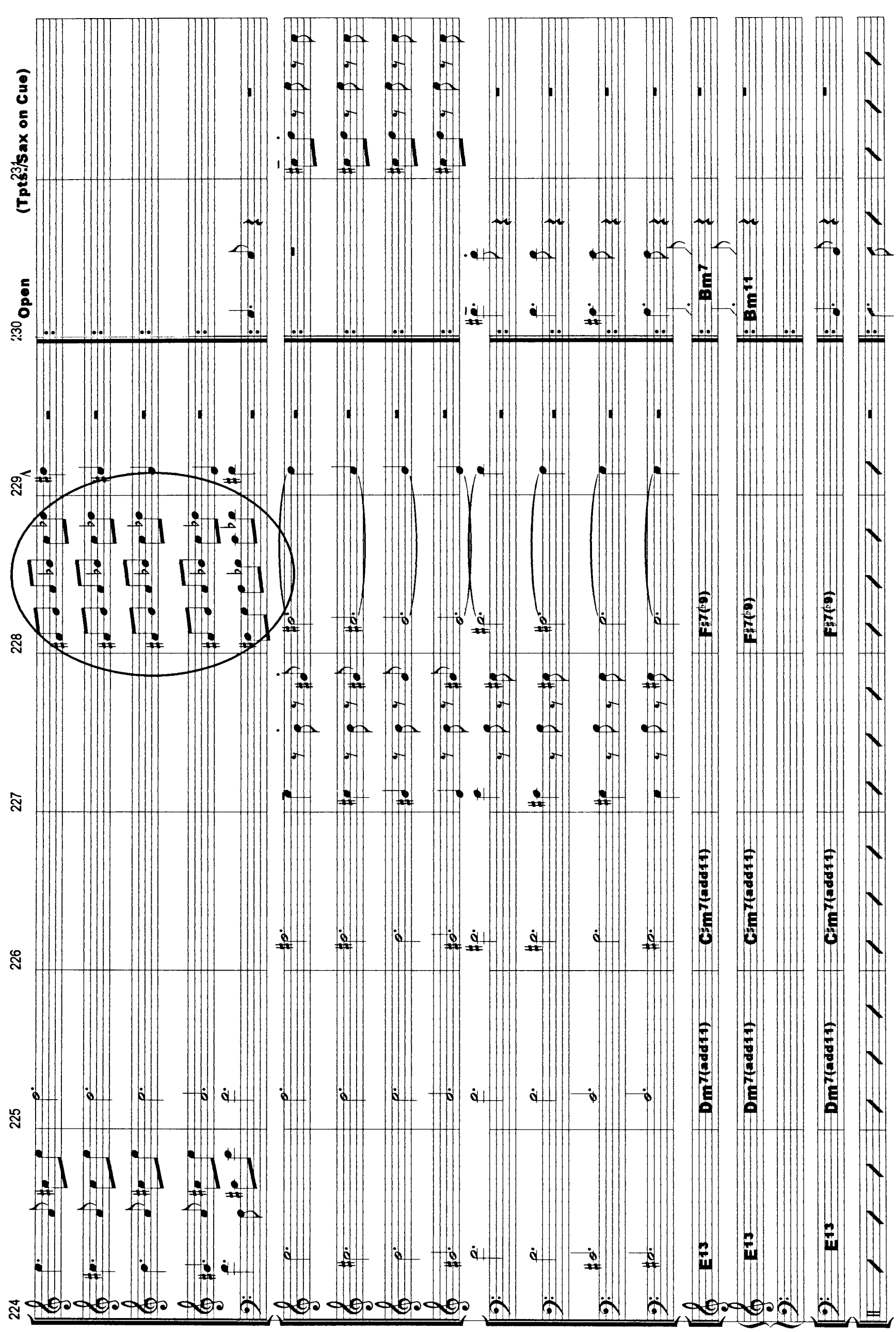


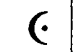

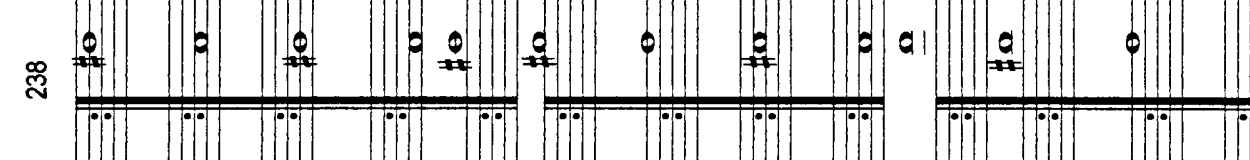

$\stackrel{\ldots}{\cdots} \frac{\|}{\cdots}$

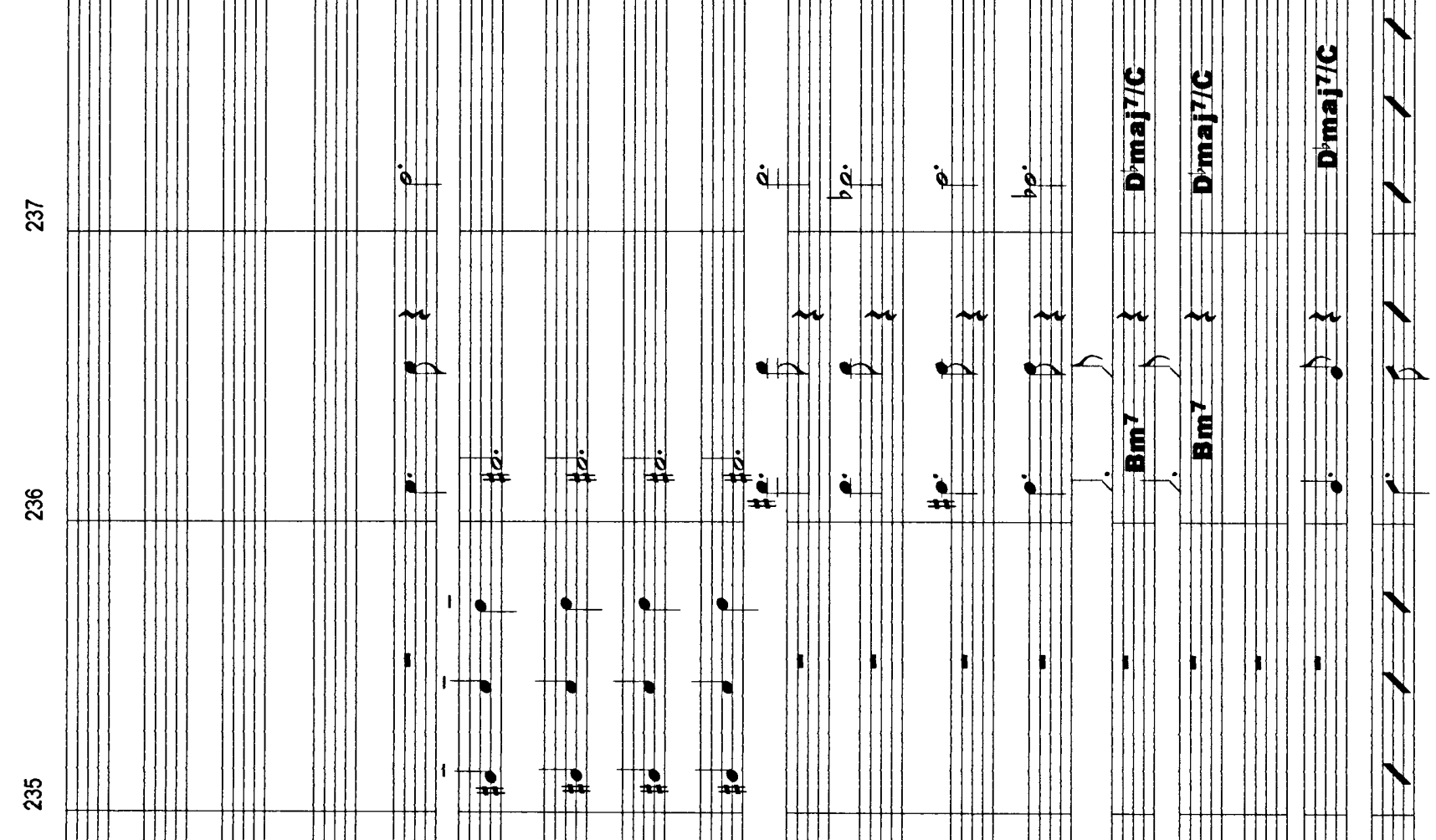

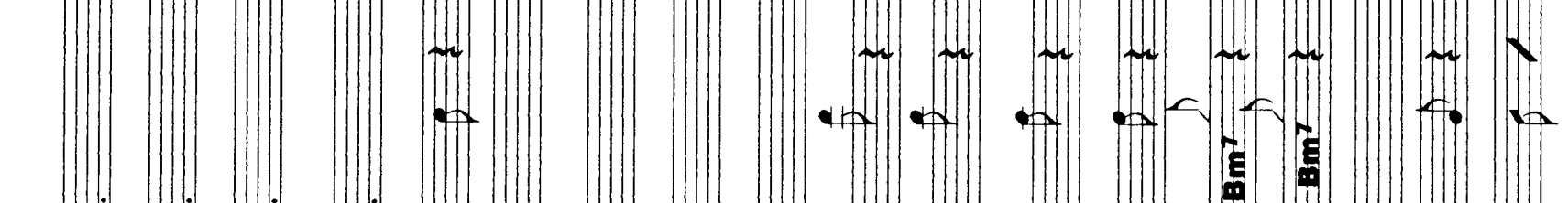

莎

号'

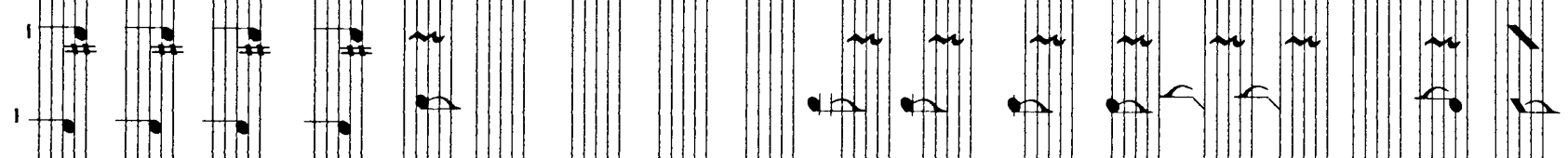

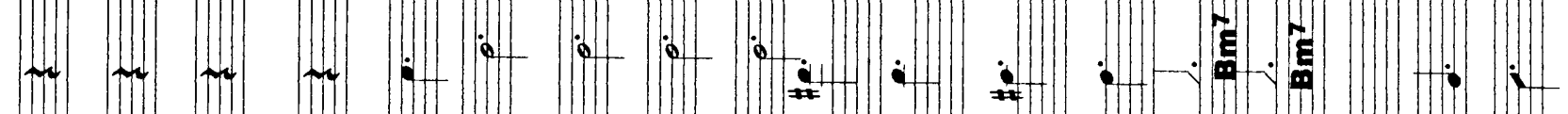

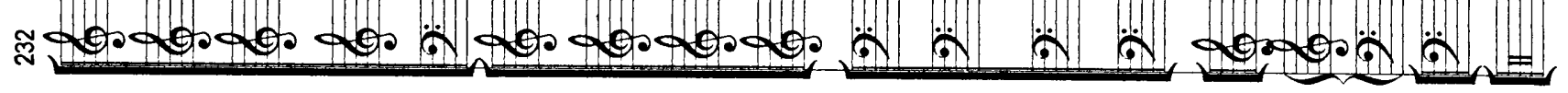

